Supporting information (73 pages)

\title{
Hydrazines and Azides via the Metal-Catalyzed Hydrohydrazination and Hydroazidation of Olefins
}

\author{
Jérôme Waser, Boris Gaspar, Hisanori Nambu and Erick M. Carreira* \\ Laboratorium für Organische Chemie, ETH Zürich, Hönggerberg HCI-H335, 8093 Zürich, \\ Switzerland \\ RECEIVED DATE; E-mail: carreira@ org.chem.ethz.ch
}


1. General Methods $\quad 3$

2. The Hydrohydrazination of Olefins $\quad 3$

$\begin{array}{ll}\text { 2.1. } & \text { Ligands and Catalysts }\end{array}$

$\begin{array}{ll}\text { 2.2. } & \text { Synthesis of Alkene Substrates }\end{array}$

2.3. The Co- and Mn- Catalyzed Hydrohydrazination of Olefins $\quad 14$

$\begin{array}{lll}\text { 2.3.1. General Procedures } & 14\end{array}$

2.3.2. Hydration of enyne 5 15

2.3.3. Hydrohydrazination Products 15

3. The Co-Catalyzed Hydroazidation of Olefins $\quad 30$

3.1. Catalysts and Azide Transfer Reagents 30

3.2. Synthesis of Alkene Substrates 33

3.3. The Hydroazidation of Olefins 35

$\begin{array}{lll}\text { 3.3.1. General Procedures } & 35\end{array}$

3.3.2. Hydroazidation Products $\quad 37$

4. Mechanistic Investigations $\quad 42$

4.1. NMR and Deuterium-Labeling Experiments $\quad 42$

4.2. $\quad$ Radical Clocks $\quad 43$

4.3. Kinetic Measurements 45

4.3.1. Methods and Formula $\quad 45$

4.3.2. Graphical Description of the Results 47

4.3.3. Kinetics Data $\quad 53$

5. Useful Transformation of Azide and Hydrazine Products 63

$\begin{array}{ll}\text { 6. Important Spectra for Characterisation } & 67\end{array}$ 


\section{General Methods}

All reactions were carried out in oven dried glassware under an atmosphere of argon or nitrogen, unless stated otherwise. For flash chromatography technical grade solvents were used, which were distilled prior to use. Alcoholic solvents for reactions were analytical grade purchased from Merck, Baker or Fluka and used without further purifications. THF, $\mathrm{Et}_{2} \mathrm{O}, \mathrm{CH}_{3} \mathrm{CN}$, toluene and $\mathrm{CH}_{2} \mathrm{Cl}_{2}$ were purified by distillation and dried by passage over activated alumina under argon atmosphere $\left(\mathrm{H}_{2} \mathrm{O}\right.$ content $<30 \mathrm{ppm}$, Karl-Fischer titration). DCE was distilled under argon from $\mathrm{CaH}_{2} . \mathrm{NEt}_{3}$ and pyridine were distilled under nitrogen from $\mathrm{KOH}$. All chemicals were purchased from Acros, Aldrich, Fluka, Merck or Lancaster and used as such unless stated otherwise.

Chromatographic purification was performed as flash chromatography using Brunschwig silica 32-63, $60 \AA$, using the solvents indicated as eluent with $0.3-0.5$ bar pressure.

TLC was performed on Merck silica gel $60 \mathrm{~F}_{254}$ TLC glass plates and visualized with UV light and permanganate stain.

Melting points were measured on a Büchi 510 melting point apparatus using open glass capillaries, the data is uncorrected.

${ }^{1} H$-NMR spectra were recorded on a VARIAN Mercury $300 \mathrm{MHz}$ spectrometer in chloroform-d, DMSO-d $\mathrm{d}_{6}$ or $\mathrm{CD}_{3} \mathrm{OD}$, all signals are reported in ppm with the internal chloroform signal at $7.26 \mathrm{ppm}$, the internal DMSO signal at $2.50 \mathrm{ppm}$ or the internal methanol signal at $3.30 \mathrm{ppm}$ as standard. The data is being reported as ( $\mathrm{s}=$ singlet, $\mathrm{d}=$ doublet, $\mathrm{t}=$ triplet, $\mathrm{q}=$ quadruplet, $\mathrm{qi}=$ quintet, $\mathrm{m}=$ multiplet or unresolved, $\mathrm{br}=$ broad signal, coupling constant(s) in Hz, integration, interpretation). NOE- ${ }^{1} H$-NMR spectra were measured on a Bruker DMX $500 \mathrm{MHz}$ spectrometer.

${ }^{2} D$-NMR spectra were recorded on a Bruker DRX $400 \mathrm{MHz}$ spectrometer in $\mathrm{CHCl}_{3}$.

${ }^{13} \mathrm{C}$-NMR spectra were recorded with ${ }^{1} \mathrm{H}$-decoupling on a VARIAN Mercury $75 \mathrm{MHz}$ spectrometer in chloroform-d, DMSO-d $\mathrm{d}_{6}$ or $\mathrm{CD}_{3} \mathrm{OD}$, all signals are reported in ppm with the internal chloroform signal at $77.0 \mathrm{ppm}$, the internal DMSO signal at $39.5 \mathrm{ppm}$ or the internal methanol signal at $49.0 \mathrm{ppm}$ as standard.

Infrared spectra were recorded on a Perkin Elmer Spectrum RX-I FT-IR spectrophotometer as thin films unless stated otherwise and are reported $\mathrm{as}^{-1}(\mathrm{w}=$ weak, $\mathrm{m}=$ medium, $\mathrm{s}=$ strong, $\mathrm{sh}=$ shoulder $)$.

REACT-IR spectra were recorded on a Mettler Toledo REACT-IR-4000 spectrophotometer and monitored using a Mettler Toledo REACT-IR 3 software.

Gas chromatographic measurements were performed on a Varian 3300 gas chromatographer or a HP 6890 Series gas chromatographer using a Supelco fused silica column $\beta$-Dex 120 (length: $30 \mathrm{~m}$, diameter: $0.25 \mathrm{~cm}$, film thickness $0.25 \mu \mathrm{m}$ ), hydrogen as carrier gas and a FID detector.

Mass spectrometric measurements were performed by the mass spectrometry service of the LOC at the ETHZ on a Finnigan TSQ 7000 ESI spectrometer for low resolution measurements and on an IONSPEC Ultima ESI-FT-ICR spectrometer at 4.7 Tesla, on an Ion Spec Ultima HR FT-ICR MS MALDI-FT-ICR MS using the DHB-tl (2,5-Dihydroxy-benzoic acid-two layers) method at 4.7 Tesla or on a EI-HIRES Micromass Autospel-ULTIMA spectrometer at $70 \mathrm{eV}$ for high resolution measurements.

Elemental analysis was performed by the Mikroelementaranalytisches Laboratorium der ETHZ.

Enantiomeric excesses were determined by chiral HPLC analysis with Merck-Hitachi D-7000 system. Solvent mixtures, conditions, retention times and columns used are given in parentheses.

Caution: Azides are potentially hazardous compounds and adequate safety measures should be taken. Reaction, especially when involving bigger quantities or heating, should be conducted behind safety shields.

\section{The Hydrohydrazination of Olefins}

\subsection{Ligands and Catalysts}

$\mathrm{Co}(\mathrm{acac})_{2}, \mathrm{CoCl}_{2} \bullet 6 \mathrm{H}_{2} \mathrm{O}, \mathrm{Co}\left(\mathrm{NO}_{3}\right)_{2} \bullet 6 \mathrm{H}_{2} \mathrm{O}$ and $\mathrm{Co}(\mathrm{OAc})_{2} \bullet 4 \mathrm{H}_{2} \mathrm{O}$ are commercially available and were used as received. $\mathrm{Co}(\mathrm{OAc})_{2}$ and $\mathrm{CoCl}_{2}$ were obtained by drying commercially available $\mathrm{Co}(\mathrm{OAc})_{2} \bullet 4 \mathrm{H}_{2} \mathrm{O}$ and $\mathrm{CoCl}_{2} \bullet 6 \mathrm{H}_{2} \mathrm{O} 5 \mathrm{~h}$ at $100{ }^{\circ} \mathrm{C}$ under high vacuo. $\mathrm{Co}(\operatorname{modp})_{2}{ }^{1}$ and $\mathrm{Co}(\mathrm{dpm})_{2}{ }^{2}$ were synthesized following reported procedures. Following a reported procedure, ${ }^{3}$ ligands 7 to 11 and 16 were prepared by condensation of the corresponding amine with salicylaldehyde (17) in the presence of triethylamine and the green $\mathrm{Co}$ (II) complexes were obtained by mixing two equivalents of the ligand with one equivalent $\mathrm{CoCl}_{2}$ in acetonitrile for $20 \mathrm{~h}$, followed by solvent removal and drying in high vacuo for at least $5 \mathrm{~h}$. The red-brown Co(III) complexes derived from ligand 12 to 16 were formed in one-pot from the free amino acids, salicylaldehyde (17) and $\mathrm{Co}\left(\mathrm{NO}_{3}\right)_{2} \bullet 6 \mathrm{H}_{2} \mathrm{O}$ as described in procedure A for Co catalyst $\mathbf{2 0 .}$

$N$-salicylidene-2-amino-isobutyrato -derivated cobalt (III) complexes

Procedure A: ${ }^{4}$ 


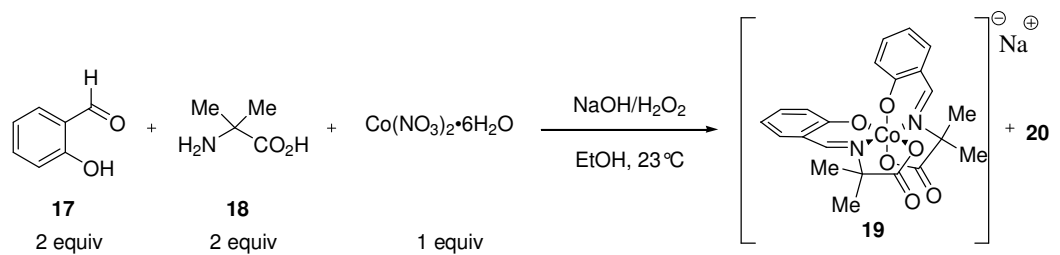

Salicylaldehyde (17) (1.06 mL, $10.0 \mathrm{mmol}, 2.00$ equiv) and 2-aminoisobutyric acid (18) (1.03 g, $10.0 \mathrm{mmol}, 2.00$ equiv) were added to a solution of sodium hydroxide $\left(0.21 \mathrm{~g}, 5.0 \mathrm{mmol}, 1.0\right.$ equiv) in ethanol $(10 \mathrm{~mL})$ at $23{ }^{\circ} \mathrm{C}$ under argon. The resulting thick yellow suspension was cooled to $0{ }^{\circ} \mathrm{C}$ and $\mathrm{Co}(\mathrm{II})$ nitrate hexahydrate $(1.5 \mathrm{~g}, 5.0 \mathrm{mmol}, 1.0$ equiv $)$ was added as a cold $(0$ $\left.{ }^{\circ} \mathrm{C}\right)$ solution in ethanol $(10 \mathrm{~mL})$ and the suspension turned to orange. After stirring $15 \mathrm{~min}$ at $0{ }^{\circ} \mathrm{C}$, hydrogen peroxide $(35 \%, 7.0$ $\mathrm{mL}$ ) was added dropwise over $20 \mathrm{~min}$, whereas the reaction mixture immediately turned dark red under gas evolution. The reaction mixture was further stirred at $0{ }^{\circ} \mathrm{C}$ for $2 \mathrm{~h}$ and then warmed to $23{ }^{\circ} \mathrm{C}$ over $3 \mathrm{~h}$. The resulting nearly clear dark red solution was filtered, the solvent was removed under reduced pressure and the residues co- evaporated with methanol $\left(2 \mathrm{x}^{2}\right)$ and $\mathrm{CH}_{2} \mathrm{Cl}_{2}$ $(2 \mathrm{x})$. The resulting dark red-brown residues were suspended in $\mathrm{Et}_{2} \mathrm{O}$, triturated for $1 \mathrm{~h}$ and filtered, the obtained fine dark redbrown powder was dried in high vacuo at $70{ }^{\circ} \mathrm{C}$ for $12 \mathrm{~h}$ to afford a dark-red brown solid $(2.04 \mathrm{~g}, 4.14 \mathrm{mmol}, 83 \%)$.

The identity of the isolated compound was established to be a mixture by NMR analysis. The catalyst was used without further purifications and several batches furnished reproducible results. No lost of activity was observed after several months when the compound was kept in dessicator at $23{ }^{\circ} \mathrm{C}$.

Procedure $\mathrm{B} \mathbf{4}^{4}$

Sodium-bis-[ $N$-salicylidene-2-amino-isobutyrato]-cobaltate(III) (19)

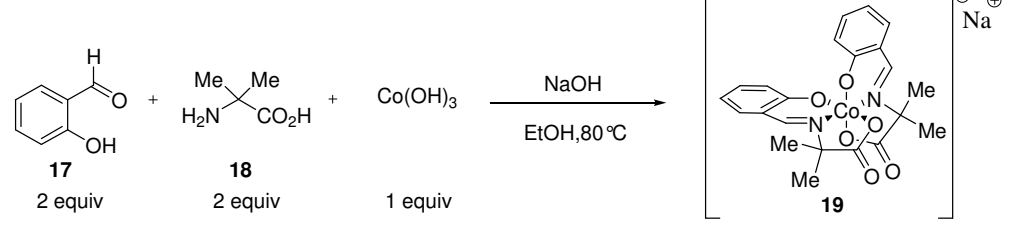

Sodium peroxide $\left(0.47 \mathrm{~g}, 6.0 \mathrm{mmol}, 1.2\right.$ equiv) was added slowly over $10 \mathrm{~min}$ to a solution of $\mathrm{Co}\left(\mathrm{NO}_{3}\right)_{2} \cdot 6 \mathrm{H}_{2} \mathrm{O}(1.5 \mathrm{~g}, 5.0$ mmol, 1.0 equiv) in water $(15 \mathrm{~mL})$, whereas black-green $\mathrm{Co}(\mathrm{OH})_{3}$ precipitated. The reaction mixture was filtered and the precipitate washed with water $(2 \times 7 \mathrm{~mL})$ and ethanol $(7 \mathrm{~mL})$. The collected $\mathrm{Co}(\mathrm{OH})_{3}$ gel in ethanol $(10 \mathrm{~mL})$ was added to a suspension of salicylaldehyde (17) $(1.1 \mathrm{~mL}, 10 \mathrm{mmol}, 2.0$ equiv) and 2-aminoisobutyric acid (18) $(1.0 \mathrm{~g}, 10 \mathrm{mmol}, 2.0 \mathrm{equiv})$ in ethanol $(10 \mathrm{~mL})$ and the resulting suspension was refluxed for $18 \mathrm{~h}$. The nearly clear dark red solution was cooled to $23{ }^{\circ} \mathrm{C}$, filtered and the solvent was removed under reduced pressure. After co-evaporating with $\mathrm{CH}_{2} \mathrm{Cl}_{2}(3 \mathrm{x})$, the dark red solid was triturated in $\mathrm{Et}_{2} \mathrm{O}(50 \mathrm{~mL})$, filtered, washed with $\mathrm{Et}_{2} \mathrm{O}(50 \mathrm{~mL})$ and dried $6 \mathrm{~h}$ in high vacuo at $70{ }^{\circ} \mathrm{C}$. The obtained complex was nearly pure $19 \cdot \mathrm{EtOH}$, but this complex proved much less active for the hydrohydrazination reaction and not active at all for the hydroazidation reaction. ${ }^{1} \mathrm{H} \mathrm{NMR}\left(\mathrm{CD}_{3} \mathrm{OD}, 300 \mathrm{MHz}\right) \delta 8.65(\mathrm{~s}, 2 \mathrm{H}$, imine $\mathrm{H}), 7.49(\mathrm{~d}, J=8.1 \mathrm{~Hz}, 2 \mathrm{H}, \mathrm{Ar} \mathrm{H}), 7.07-7.01(\mathrm{~m}, 2 \mathrm{H}$, $\mathrm{Ar} \mathrm{H}), 6.74(\mathrm{~d}, J=8.7 \mathrm{~Hz}, 2 \mathrm{H}, \mathrm{Ar} \mathrm{H}), 6.58-6.53(\mathrm{~m}, 2 \mathrm{H}, \mathrm{Ar} \mathrm{H}), 3.48\left(\mathrm{q}, J=7.2 \mathrm{~Hz}, 2 \mathrm{H}\right.$, ethanol- $\left.\mathrm{CH}_{2}\right), 1.88\left(\mathrm{~s}, 6 \mathrm{H}, \mathrm{CH}_{3}\right), 1.87(\mathrm{~s}$, $\left.6 \mathrm{H}, \mathrm{CH}_{3}\right), 1.17\left(\mathrm{t}, J=7.2 \mathrm{~Hz}, 3 \mathrm{H}\right.$, ethanol- $\left.\mathrm{CH}_{3}\right) ;{ }^{13} \mathrm{C}$ NMR $\left(\mathrm{CD}_{3} \mathrm{OD}, 75 \mathrm{MHz}\right) \delta 188.1,165.3,164.6,135.4,134.2,122.5,120.5$, 115.4, 71.2, 66.5, 29.0, 27.8, 15.2;IR (KBr pellets) v $3412(\mathrm{~m}), 3051$ (w), $2974(\mathrm{~m}), 2930$ (w), $2870(\mathrm{w}), 1641(\mathrm{~s}), 1602(\mathrm{~s}), 1555$ (m), $1532(\mathrm{~m}), 1466(\mathrm{~s}), 1450(\mathrm{~s}), 1383(\mathrm{~s}), 1350(\mathrm{~s}), 1321(\mathrm{~m}), 1205(\mathrm{~m}), 1173(\mathrm{~m}), 1146(\mathrm{~m}), 1125(\mathrm{~m}), 1108(\mathrm{w}), 1030(\mathrm{w}), 994$ (w), $962(\mathrm{w}), 901(\mathrm{~m}), 845(\mathrm{w}), 803(\mathrm{w}), 752(\mathrm{~m}), 735(\mathrm{~m}), 706(\mathrm{w}), 656(\mathrm{w}), 624(\mathrm{w}), 587(\mathrm{w}), 556(\mathrm{w}), 533(\mathrm{w}), 514(\mathrm{w}), 464$ (w), 407 (w);HRMS (ESI) calcd for $\mathrm{C}_{22} \mathrm{H}_{22} \mathrm{CoN}_{2} \mathrm{Na}_{2} \mathrm{O}_{6}{ }^{+}$(M+Na): 515.0600, found 515.0608; calcd for $\mathrm{C}_{22} \mathrm{H}_{22} \mathrm{CoN}_{2} \mathrm{O}_{6}{ }^{-}(\mathrm{M}-\mathrm{Na})$ : 469.0815, found 469.0821.

\section{Procedure C:}

[N-salicylidene-2-amino-isobutyrato]-[2-amino-isobutyrato]-cobalt (III) (20)

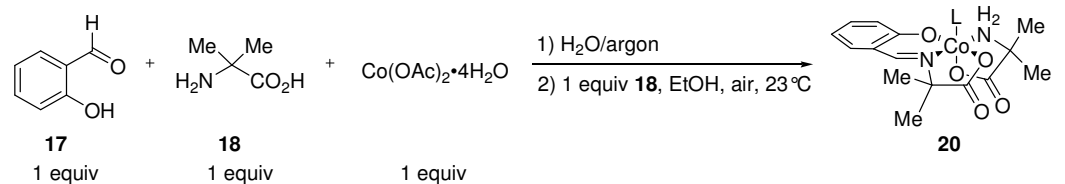

$\mathrm{Co}(\mathrm{OAc})_{2} \bullet 4 \mathrm{H}_{2} \mathrm{O}(12.5 \mathrm{~g}, 50.2 \mathrm{mmol}, 1.00$ equiv) was added to a suspension of salicylaldehyde (17) $(5.2 \mathrm{~mL}, 50 \mathrm{mmol}, 1.0$ equiv) and 2-aminoisobutyric acid (18) $\left(5.1 \mathrm{~g}, 50 \mathrm{mmol}, 1.0\right.$ equiv) in water $(50 \mathrm{~mL})$ at $23{ }^{\circ} \mathrm{C}$ under argon and the reaction mixture was heated to reflux. After refluxing for $2 \mathrm{~h}$, a thick suspension was obtained. The reaction mixture was filtered, the solid washed with water $(60 \mathrm{~mL})$ and dried $12 \mathrm{~h}$ in high vacuo.

The isolated solid $(1.0 \mathrm{~g})$ was then suspended in water $(12 \mathrm{~mL})$ and ethanol $(30 \mathrm{~mL})$ under air and 2-aminoisobutyric acid (18) $(0.32 \mathrm{~g}, 3.1 \mathrm{mmol})$ was added. The reaction mixture was stirred under air at $23{ }^{\circ} \mathrm{C}$ and slowly turned to a dark red solution. After $5 \mathrm{~h}$, the dark red solution was filtered and the solvent was removed under reduced pressure. The residues were co-evaporated with $\mathrm{CH}_{2} \mathrm{Cl}_{2}(2 \mathrm{x})$, dissolved in ethanol, filtered, the solvent was removed under reduced pressure and the isolated product was dried $6 \mathrm{~h}$ 
in high vacuo at $80{ }^{\circ} \mathrm{C}$. Further purification was achieved via column chromatography $\left(\mathrm{CH}_{2} \mathrm{Cl}_{2} / \mathrm{MeOH}\right.$, solvent gradient $1: 0$ to 10:1 to $1: 1)$ to afford $20(0.92 \mathrm{~g}, 2.3 \mathrm{mmol}, 77 \%)$ as a dark red solid. The complex obtained by this procedure contained pure $\mathbf{2 0}$ $(\mathrm{L}=\mathrm{MeOH})$ as a mixture of isomers $(2: 1)$. It shows a slightly increased activity compared to the complex obtained following procedure A. ${ }^{1} \mathrm{H}$ NMR $\left(\mathrm{CD}_{3} \mathrm{OD}, 300 \mathrm{MHz}\right) \delta 8.61$ (s, $1 \mathrm{H}(33 \%)$, imine $\mathrm{H} 1$. isomer), 8.30 (s, $1 \mathrm{H}(66 \%)$, imine $\mathrm{H} 2$. isomer), 7.567.48 (m, 2H, Ar H), 7.29-7.19 (m, 2H, Ar H), 7.09 (d, J=8.7 Hz, 2H, Ar H), 6.70-6.62 (m, 2H, Ar H), $1.84\left(\mathrm{~s}, 3 \mathrm{H}(33 \%), \mathrm{CH}_{3}\right.$ 1.isomer), 1.82 (s, $3 \mathrm{H}(33 \%), \mathrm{CH}_{3}$ 1.isomer), 1.79 (s, $3 \mathrm{H}(66 \%), \mathrm{CH}_{3}$ 2. isomer), 1.70 (s, 3H (33\%), $\mathrm{CH}_{3} 1$. isomer), 1.67 (s, $3 \mathrm{H}$ (66\%), $\mathrm{CH}_{3}$ 2. isomer), 1.62 (s, $3 \mathrm{H}(33 \%), \mathrm{CH}_{3} 1$ isomer), 1.32 (s, $3 \mathrm{H}(66 \%), \mathrm{CH}_{3} 2$. isomer), 1.26 (s, $3 \mathrm{H}(66 \%), \mathrm{CH}_{3} 2$. isomer); ${ }^{13} \mathrm{C}$ NMR (DMSO-d 6 , $\left.75 \mathrm{MHz}\right) \delta 185.5,184.3,183.8,183.2,166.3,165.8,163.8,163.5,157.6,134.7,134.6,133.6,121.9,121.7$, 118.6, 118.2, 114.4, 114.0, 69.6, 69.4, 60.3, 58.2, 29.0, 28.3, 28.2, 27.9, 27.1, 26.6; IR (KBr pellets) v $3410(\mathrm{~m}), 3242(\mathrm{~m}), 3130$ (m), $2978(\mathrm{~m}), 1661$ (s, sh), 1644 (s), 1600 (s), 1546 (s, sh), 1467 (m), 1450 (s), 1387 (s), 1345 (s), 1310 (m), 1205 (m), $1174(\mathrm{~m})$, $1147(\mathrm{~m}), 1128(\mathrm{w}), 1032(\mathrm{w}), 995(\mathrm{w}), 901(\mathrm{~m}), 847(\mathrm{w}), 807(\mathrm{w}), 756(\mathrm{~m}), 705(\mathrm{w}), 661(\mathrm{w}), 626(\mathrm{w}), 588(\mathrm{w}), 552(\mathrm{w}), 530$ (w), $468(\mathrm{w}), 419(\mathrm{w})$; HRMS (MALDI) calcd for $\mathrm{C}_{15} \mathrm{H}_{20} \mathrm{CoN}_{2} \mathrm{O}_{5}{ }^{+}(\mathrm{M}+\mathrm{H})$ : 367.0704, found 367.0695; calcd for $\mathrm{C}_{15} \mathrm{H}_{19} \mathrm{CoN}_{2} \mathrm{O}_{5} \mathrm{Na}^{+}(\mathrm{M}+\mathrm{Na})$ : 389.0524 , found 389.0516.

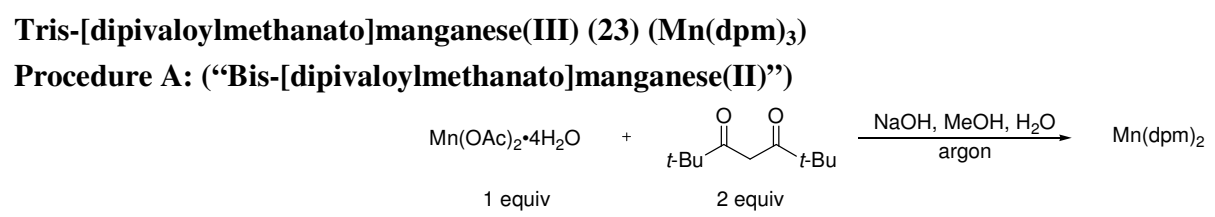

Following a slightly modified literature procedure, ${ }^{2}$ 2,2,4,4-tetramethylhept-3,5-dione $(0.96 \mathrm{~mL}, 4.6 \mathrm{mmol}, 2.0$ equiv, freshly distilled) was added to a solution of manganese(II)diacetate tetrahydrate ( $0.56 \mathrm{~g}, 2.3 \mathrm{mmol}, 1.0$ equiv) in methanol (4.5 mL, distilled, degassed (two freeze-thaw cycles)) under argon and the resulting yellow solution was stirred at $23{ }^{\circ} \mathrm{C}$ with argon bubbling for $5 \mathrm{~min}$. A solution of sodium hydroxide $(0.18 \mathrm{~g}, 4.6 \mathrm{mmol}, 2.0 \mathrm{eq})$ in water $(1.2 \mathrm{~mL}$, deionized, degassed (20 min argon bubbling)) was then added, whereas a yellow solid immediately precipitated. The solid was filtred under argon, washed with methanol/water 2:1 (20 mL) and dried $15 \mathrm{~h}$ in high vacuo over $\mathrm{P}_{2} \mathrm{O}_{5}$, whereas a color change to olive green was observed, which shows a partial convertion to $\mathrm{Mn}(\mathrm{dpm})_{3}(\mathbf{2 3})$. The obtained olive green $\mathrm{Mn}(\mathrm{dpm})_{2}(0.69 \mathrm{~g}, 1.6 \mathrm{mmol}, 70 \%)$ was used without further purification

Procedure B:

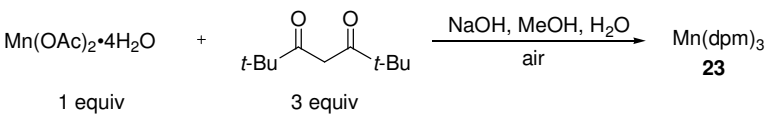

2,2,4,4-Tetramethylhept-3,5-dione (1.3 mL, $6.1 \mathrm{mmol}, 3.0$ equiv) was added to a solution of manganese(II)diacetate tetrahydrate $(0.50 \mathrm{~g}, 2.0 \mathrm{mmol}, 1.0$ equiv) in methanol $(4.5 \mathrm{~mL})$ under air and the resulting yellow-green solution was stirred at 23 ${ }^{\circ} \mathrm{C}$. A solution of sodium hydroxide $(0.25 \mathrm{~g}, 6.1 \mathrm{mmol}, 3.0 \mathrm{eq})$ in water $(1.2 \mathrm{~mL}$, deionized) was then added, whereas a green solid immediately precipitated. After diluting with methanol $(5.5 \mathrm{~mL})$, the reaction mixture was stirred at $23{ }^{\circ} \mathrm{C}$ under air for $13 \mathrm{~h}$ and filtered. The green-brown solid was dried in high vacuo at $60^{\circ} \mathrm{C}$ for $4 \mathrm{~h}$, dissolved in hot isopropanol $(15 \mathrm{~mL}), \mathrm{Mn}(\mathrm{dpm})_{3}$ (23) precipitated partially upon cooling, the precipitation was completed by adding water $(3 \mathrm{~mL})$ and the suspension was filtered. The resulting solid was suspended in pentane $(10 \mathrm{~mL})$ and the resulting suspension was filtered, removing some brown-red impurities. The pentane was removed under reduced pressure and the resulting olive green solid was dried under high vacuo for $12 \mathrm{~h}$ to give $\mathrm{Mn}(\mathrm{dpm})_{3}(\mathbf{2 3})$ as a green powder $(0.76 \mathrm{~g}, 1.2 \mathrm{mmol}, 62 \%$ yield $)$.

The complexes synthesized following either procedure $\mathbf{A}$ or $\mathbf{B}$ showed similar activities for the hydrohydrazination reaction. The complexe obtains from B was characterized. Mp 164-165 ${ }^{\circ} \mathrm{C}$; IR v 2964 (s), 2867 (m), 1593 (s), 1572 (s), 1496 (s), 1402 (s), $1358(\mathrm{~s}), 1284(\mathrm{~m}), 1246$ (m), 1222 (s), $1176(\mathrm{~m}), 1134(\mathrm{~s}), 1023(\mathrm{w}), 958(\mathrm{w}), 936$ (w), $871(\mathrm{~s}), 792$ (m), 760 (w), 740 (w), 641 (m); HRMS (MALDI) calcd for $\mathrm{Mn}(\mathrm{dpm})_{2}{ }^{+}: 421.2145$, found 421.2156; Anal. calcd for $\mathrm{C}_{33} \mathrm{H}_{57} \mathrm{MnO}_{6}: \mathrm{C}, 65.54 ; \mathrm{H}, 9.50$. Found: C, 65.62; H, 9.49.

\subsection{Synthesis of Alkene Substrates}

\section{2-Methyl-hex-5-en-3-yn-2-ol (70) and 4-Methoxy-benzoic acid 1,1-dimethyl-pent-4-en-2-ynyl ester (5)}
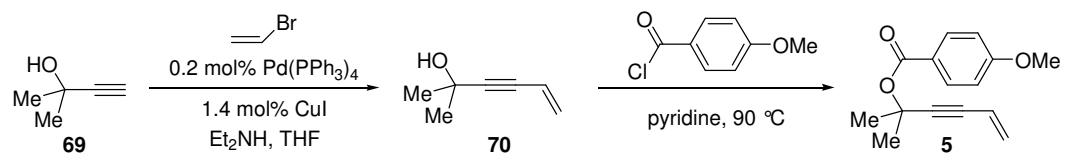

2-Methyl-hex-5-en-3-yn-2-ol (70) ${ }^{5}$

Following a reported procedure, ${ }^{6} \mathrm{CuI}\left(0.14 \mathrm{~g}, 0.72 \mathrm{mmol}, 0.014\right.$ equiv) and $\mathrm{Pd}\left(\mathrm{PPh}_{3}\right)_{4}(0.14 \mathrm{~g}, 0.12 \mathrm{mmol}, 0.0023$ equiv $)$ were dissolved in diethylamine (freshly distilled over $\mathrm{KOH}, 25 \mathrm{~mL}$ ) at $10{ }^{\circ} \mathrm{C}$ under argon. 2-Methyl-3-in-2-ol (69) (freshly distilled, 5.0 $\mathrm{mL}, 52 \mathrm{mmol}, 1.0$ equiv) and vinyl bromide (1 M in THF, $65 \mathrm{~mL}, 65 \mathrm{mmol}, 1.3$ equiv) were added dropwise and the resulting suspension stirred at $23{ }^{\circ} \mathrm{C}$ for $2.5 \mathrm{~h}$. The reaction mixture was then poured into water $(100 \mathrm{~mL})$ at $0{ }^{\circ} \mathrm{C}$ and extracted with $\mathrm{Et}_{2} \mathrm{O}$ 
$(3 \times 125 \mathrm{~mL})$, the combined organic layers were washed with $2 \mathrm{M} \mathrm{HCl}(2 \times 50 \mathrm{~mL})$, dried over $\mathrm{Na}_{2} \mathrm{SO}_{4}$ and concentrated under reduced pressure. The crude mixture was distilled $\left(p=20 \mathrm{mbar}, b p=58^{\circ} \mathrm{C}\right)$ to afford $70(4.84 \mathrm{~g}, 43.5 \mathrm{mmol}, 84 \%)$ as a colorless liquid. ${ }^{1} \mathrm{H} \mathrm{NMR}\left(\mathrm{CDCl}_{3}, 300 \mathrm{MHz}\right) \delta 5.81(\mathrm{dd}, J=17.4,10.9 \mathrm{~Hz}, 1 \mathrm{H}$, alkene $\mathrm{H}), 5.62(\mathrm{dd}, J=17.7,2.5 \mathrm{~Hz}, 1 \mathrm{H}$, alkene $\mathrm{H}), 5.46$ (dd, $J=10.9,2.5 \mathrm{~Hz}, 1 \mathrm{H}$, alkene $\mathrm{H}), 1.97(\mathrm{bs}, \mathrm{OH}), 1.54\left(\mathrm{~s}, 6 \mathrm{H},\left(\mathrm{CH}_{3}\right)_{2} \mathrm{C}\right) ;{ }^{13} \mathrm{C} \mathrm{NMR}\left(\mathrm{CDCl}_{3}, 75 \mathrm{MHz}\right) \delta 126.8,116.6,94.4,80.7$, 65.4, 31.3; IR v 3350 (s), $3101(w), 3011(w), 2982(\mathrm{~s}), 2933$ (w), $2871(\mathrm{w}), 1844$ (w), 1609 (w), 1455 (w), $1411(\mathrm{~m}), 1376(\mathrm{~m})$, $1363(\mathrm{~m}), 1240$ (s), 1165 (s), 995 (w), 973 (m), 944 (s), $846(\mathrm{~m}), 678(\mathrm{w}), 551(\mathrm{w})$.

\section{4-Methoxy-benzoic acid 1,1-dimethyl-pent-4-en-2-ynyl ester (5)}

2-Methyl-hex-5-en-3-yn-2-ol (70) (2.24 g, $20.2 \mathrm{mmol}, 1.00$ equiv) was dissolved in pyridine (distilled, $10 \mathrm{~mL})$ at $23{ }^{\circ} \mathrm{C}$ under argon. The reaction mixture was cooled to $0{ }^{\circ} \mathrm{C}$ and anisoyl chloride $(3.5 \mathrm{~mL}, 26 \mathrm{mmol}, 1.3$ equiv) was added dropwise. The resulting suspension was heated to $90{ }^{\circ} \mathrm{C}$ for $14 \mathrm{~h}$, cooled to $0{ }^{\circ} \mathrm{C}$ and quenched with water $(10 \mathrm{~mL})$. The mixture was diluted with water $(30 \mathrm{~mL})$ and extracted with $\mathrm{Et}_{2} \mathrm{O}(3 \times 30 \mathrm{~mL})$. The combined organic layers were washed with $2 \mathrm{M} \mathrm{HCl}(3 \times 50 \mathrm{~mL})$, sat. $\mathrm{NaHCO}_{3}(3 \times 30 \mathrm{~mL})$, brine $(2 \times 30 \mathrm{~mL})$, dried over $\mathrm{Na}_{2} \mathrm{SO}_{4}$ and the solvent removed under reduced pressure. The crude mixture was purified by column chromatography (AcOEt/hexane 1:13) to afford $\mathbf{5}(4.40 \mathrm{~g}, 17.9 \mathrm{mmol}, 89 \%)$ as a colorless oil. Enyne $\mathbf{5}$ polymerised slowly when kept in the air at $23{ }^{\circ} \mathrm{C}$, but is stable for months when kept at $-20{ }^{\circ} \mathrm{C}$ under argon. ${ }^{1} \mathrm{H} \mathrm{NMR}(\mathrm{CDCl} 3,300$ MHz) $\delta 7.97(\mathrm{dd}, J=7.2,1.9 \mathrm{~Hz}, 2 \mathrm{H}, \mathrm{Ar} \mathrm{H}), 6.90(\mathrm{dd}, J=7.2,1.9 \mathrm{~Hz}, 2 \mathrm{H}, \mathrm{Ar} \mathrm{H}), 5.82(\mathrm{dd}, J=17.7,10.9 \mathrm{~Hz}, 1 \mathrm{H}$, alkene H), 5.64 $(\mathrm{dd}, J=17.7,2.2 \mathrm{~Hz}, 1 \mathrm{H}$, alkene $\mathrm{H}), 5.46(\mathrm{dd}, J=10.9,2.2 \mathrm{~Hz}, 1 \mathrm{H}$, alkene $\mathrm{H}), 3.85\left(\mathrm{~s}, 3 \mathrm{H}, \mathrm{OCH}_{3}\right), 1.81\left(\mathrm{~s}, 6 \mathrm{H},(\mathrm{CH})_{2} \mathrm{COC}\right) ;{ }^{13} \mathrm{C}$ NMR $\left(\mathrm{CDCl}_{3}, 75 \mathrm{MHz}\right) \delta 164.4,163.0,131.5,127.1,123.3,116.7,113.4,90.9,82.7,72.5,55.4,29.2 ; \mathrm{IR} v 2987(\mathrm{~m}), 2938(\mathrm{~m})$, 2840 (w), 2044 (w), 1922 (w), 1849 (w), 1719 (s), 1606 (s), 1511 (s), 1465 (m), 1420 (w), $1364(\mathrm{~m}), 1316(\mathrm{~m}), 1283$ (s), 1257 (s), 1170 (s), 1141 (s), 1097 (s), 1030 (m), 921 (m), $848(\mathrm{~m}), 770$ (s), 697 (m); HRMS(EI) calcd for $\mathrm{C}_{15} \mathrm{H}_{16} \mathrm{O}_{3}{ }^{+}$(M) 244.1099, found 244.1090; Anal. calcd for $\mathrm{C}_{15} \mathrm{H}_{16} \mathrm{O}_{3}$ : C, 73.75; H, 6.60. Found: C, 73.83; H, 6.79.

2-Vinyl-furan $(\mathbf{7 2})^{7}$

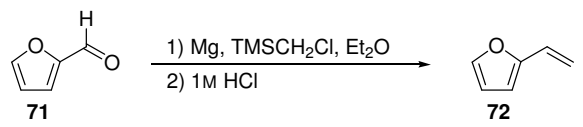

Following a slightly modified reported procedure, ${ }^{7 \mathrm{a}} \mathrm{Mg}$ (powder, $2.0 \mathrm{~g}, 82 \mathrm{mmol}, 1.5$ equiv) was suspended in $\operatorname{dry~}_{2} \mathrm{Et}_{2} \mathrm{O}(5 \mathrm{~mL})$ at $23{ }^{\circ} \mathrm{C}$ under argon and $\mathrm{TMSCH}_{2} \mathrm{Cl}\left(9.7 \mathrm{~mL}, 69 \mathrm{mmol}, 1.2\right.$ equiv) was added dropwise diluted with $\mathrm{Et}_{2} \mathrm{O}(40 \mathrm{~mL})$ over $30 \mathrm{~min}$. The reaction mixture was heated to reflux $\left(45^{\circ} \mathrm{C}\right)$ and dibromoethane $(0.10 \mathrm{~mL})$ was added. After $2 \mathrm{~h}$ and $11 \mathrm{~h}$, further portions of dibromoethane were added $(2 \times 0.10 \mathrm{~mL})$. After a total refluxing time of $18 \mathrm{~h}$, the Grignard reaction was finished and the reaction mixture was cooled to $0{ }^{\circ} \mathrm{C}$. A solution of furfuraldehyde (71) $\left(5.0 \mathrm{~mL}, 60 \mathrm{mmol}, 1.0\right.$ equiv) in $\mathrm{Et}_{2} \mathrm{O}(60 \mathrm{~mL})$ was added over $1 \mathrm{~h}$. After stirring $4 \mathrm{~h}$ at $0{ }^{\circ} \mathrm{C}$ and $14 \mathrm{~h}$ at $23{ }^{\circ} \mathrm{C}$, the reaction mixture was quenched with sat. $\mathrm{NH}_{4} \mathrm{Cl}(40 \mathrm{~mL})$ at $0{ }^{\circ} \mathrm{C}$, the layers were separated and the water layer extracted with $\mathrm{Et}_{2} \mathrm{O}(2 \times 50 \mathrm{~mL})$. The combined organic layers were washed with sat. $\mathrm{NaHCO}_{3}(2 \times 30 \mathrm{~mL})$ and brine $(30 \mathrm{~mL})$, dried over $\mathrm{Na}_{2} \mathrm{SO}_{4}$ and the solvent was removed $\left(p=100 \mathrm{mbar}, T=40^{\circ} \mathrm{C}\right)$.

The residues were dissolved in $\mathrm{Et}_{2} \mathrm{O}(20 \mathrm{~mL})$ and the reaction mixture was mixed vigorously together with aqueous $1 \mathrm{M} \mathrm{HCl}$ $(20 \mathrm{~mL})$ for $75 \mathrm{~min}$ until no alcohol was present by TLC (AcOEt/hexane 1:5). The layers were separated, the water layer extracted with $\mathrm{Et}_{2} \mathrm{O}(2 \times 20 \mathrm{~mL})$, the combined organic layers were washed with sat. $\mathrm{NaHCO}_{3}(20 \mathrm{~mL})$ and brine $(20 \mathrm{~mL})$ and dried over $\mathrm{Na}_{2} \mathrm{SO}_{4}$. After filtration, 4-tert-butyl catechol was added $(0.10 \mathrm{~g})$ and the solvent was removed $\left(p=700 \mathrm{mbar}, T=40{ }^{\circ} \mathrm{C}\right)$ under nitrogen. Destillation of the crude product $\left(p=170\right.$ mbar, $\left.b p=50-55{ }^{\circ} \mathrm{C}\right)$ under nitrogen affords 2-vinylfuran (72) in two fractions (1.fraction: $2.2 \mathrm{~g}, 46 \%$ 72, 2.fraction $4.4 \mathrm{~g}, 55 \%$ 72, total 72: $3.4 \mathrm{~g}, 37 \mathrm{mmol}, 61 \%$ ) as a mixture with $\mathrm{TMSOH}$ and $\mathrm{Et}_{2} \mathrm{O}$. This mixture was kept at $-20{ }^{\circ} \mathrm{C}$ protected from light over 4-tert-butyl catechol and was used directly as such in the hydrohydrazination reaction. ${ }^{1} \mathrm{H} \mathrm{NMR}\left(\mathrm{CDCl}_{3}, 300 \mathrm{MHz}\right) \delta 7.35(\mathrm{~d}, J=1.8 \mathrm{~Hz}, 1 \mathrm{H}$, furan $\mathrm{H}), 6.51(\mathrm{dd}, J=17.5,11.3 \mathrm{~Hz}, 1 \mathrm{H}$, vinyl H), $6.37(\mathrm{dd}, J=$ $3.3,1.8 \mathrm{~Hz}, 1 \mathrm{H}$, furan $\mathrm{H}), 6.26(\mathrm{~d}, J=3.3 \mathrm{~Hz}, 1 \mathrm{H}$, furan $\mathrm{H}), 5.66(\mathrm{dd}, J=17.5,1.3 \mathrm{~Hz}, 1 \mathrm{H}$, vinyl $\mathrm{H}), 5.16(\mathrm{dd}, J=11.3,1.3 \mathrm{~Hz}$, $1 \mathrm{H}$, vinyl $\mathrm{H}) ;{ }^{13} \mathrm{C} \mathrm{NMR}\left(\mathrm{CDCl}_{3}, 75 \mathrm{MHz}\right) \delta 153.1,141.8,124.9,112.1,111.1,107.9$.

\section{3-Vinyl-furan (74) ${ }^{7 \mathrm{c}}$}

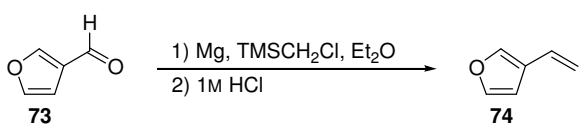

Following a slightly modified reported procedure, ${ }^{7 \mathrm{a}} \mathrm{Mg}$ (powder, $0.38 \mathrm{~g}, 16 \mathrm{mmol}, 1.5$ equiv) was suspended in $\mathrm{dry}_{\mathrm{Et}} \mathrm{O}(1$ $\mathrm{mL})$ at $23{ }^{\circ} \mathrm{C}$ under argon and $\mathrm{TMSCH}_{2} \mathrm{Cl}\left(1.7 \mathrm{~mL}, 12 \mathrm{mmol}, 1.1\right.$ equiv) was added dropwise diluted with $\mathrm{Et}_{2} \mathrm{O}(7 \mathrm{~mL}) \mathrm{over} 30$ min. The reaction mixture was heated to reflux $\left(45^{\circ} \mathrm{C}\right)$ and dibromoethane $(50 \mu \mathrm{L})$ was added. After a total refluxing time of 19 $\mathrm{h}$, the Grignard reaction was finished and the reaction mixture was cooled to $0{ }^{\circ} \mathrm{C}$. A solution of furan-3-carbaldehyde (73) $(1.0 \mathrm{~g}$, $11 \mathrm{mmol}, 1.0$ equiv) in $\mathrm{Et}_{2} \mathrm{O}(12 \mathrm{~mL})$ was added over $15 \mathrm{~min}$. After stirring $4 \mathrm{~h}$ at $0{ }^{\circ} \mathrm{C}$ and $14 \mathrm{~h}$ at $23{ }^{\circ} \mathrm{C}$, the reaction mixture was quenched with sat. $\mathrm{NH}_{4} \mathrm{Cl}(8 \mathrm{~mL})$ at $0{ }^{\circ} \mathrm{C}$, the layers were separated and the water layer extracted with $\mathrm{Et}_{2} \mathrm{O}(2 \times 10 \mathrm{~mL})$. The combined organic layers were washed with sat. $\mathrm{NaHCO}_{3}(2 \times 10 \mathrm{~mL})$ and brine $(10 \mathrm{~mL})$, dried over $\mathrm{Na}_{2} \mathrm{SO}_{4}$ and the solvent was removed $\left(p=100\right.$ mbar, $\left.T=40^{\circ} \mathrm{C}\right)$.

The residues were dissolved in $\mathrm{Et}_{2} \mathrm{O}(4 \mathrm{~mL})$ and the reaction mixture was mixed vigorously together with aqueous $1 \mathrm{M} \mathrm{HCl}(4$ $\mathrm{mL}$ ) for $60 \mathrm{~min}$ until no alcohol was present by TLC (AcOEt: hexane 1:5). The layers were separated, the water layer extracted with $\mathrm{Et}_{2} \mathrm{O}(2 \times 4 \mathrm{~mL})$, the combined organic layers were washed with sat. $\mathrm{NaHCO}_{3}(5 \mathrm{~mL})$ and brine $(5 \mathrm{~mL})$ and dried over $\mathrm{Na}_{2} \mathrm{SO}_{4}$. After filtration, 4-tert-butyl catechol was added $(0.05 \mathrm{~g})$ and the solvent was removed $\left(p=700 \mathrm{mbar}, T=40{ }^{\circ} \mathrm{C}\right)$ under 
nitrogen. Distillation of the crude product ( $\left.p=150 \mathrm{mbar}, b p=40-45^{\circ} \mathrm{C}\right)$ under nitrogen gives 3-vinylfuran $(\mathbf{7 4})(0.50 \mathrm{~g}, 41 \% \mathrm{74}$, total 74: $0.21 \mathrm{~g}, 2.2 \mathrm{mmol}, 21 \%$ ) as a mixture with $\mathrm{TMSOH}$ and $\mathrm{Et}_{2} \mathrm{O}$. This mixture was kept at $-20^{\circ} \mathrm{C}$ protected from light over 4-tert-butyl catechol and was used directly as such in the hydrohydrazination reaction. ${ }^{1} \mathrm{H} \mathrm{NMR}\left(\mathrm{CDCl}_{3}, 300 \mathrm{MHz}\right) \delta 7.42(\mathrm{~s}, 1 \mathrm{H}$, furan $\mathrm{H}), 7.37(\mathrm{~m}, 1 \mathrm{H}$, furan $\mathrm{H}), 6.57$ (dd, $J=17.4,10.9 \mathrm{~Hz}, 1 \mathrm{H}$, vinyl H), $6.56(\mathrm{~d}, J=0.9 \mathrm{~Hz}, 1 \mathrm{H}$, furan $\mathrm{H}), 5.46(\mathrm{dd}, J=17.4$, $0.9 \mathrm{~Hz}, 1 \mathrm{H}$, vinyl $\mathrm{H}), 5.14(\mathrm{dd}, J=10.9,1.3 \mathrm{~Hz}, 1 \mathrm{H}$, vinyl $\mathrm{H}) ;{ }^{13} \mathrm{C} \mathrm{NMR}\left(\mathrm{CDCl}_{3}, 75 \mathrm{MHz}\right) \delta 143.3,140.4,126.4,124.7,113.2$, 107.1.

2-Vinyl-thiophene (76) ${ }^{8}$

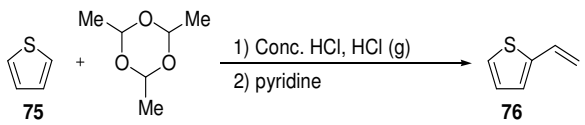

Following a slightly modified reported procedure, ${ }^{8 \mathrm{a}}$ concentrated $\mathrm{HCl}(37 \%, 15 \mathrm{~mL})$ was added at $0{ }^{\circ} \mathrm{C}$ under argon to a mixture of thiophene (75) $(16 \mathrm{~mL}, 0.20 \mathrm{~mol}, 1.0$ equiv) and paraldehyde $(8.9 \mathrm{~mL}, 67 \mathrm{mmol}, 0.33$ equiv). Gaseous $\mathrm{HCl}$ was bubbled through the reaction mixture at $10^{\circ} \mathrm{C}$ for $1 \mathrm{~h}$. The reaction mixture was then poured into crushed ice $(15 \mathrm{~g}), \mathrm{Et}_{2} \mathrm{O}(15 \mathrm{~mL})$ was added, the layers were separated, the water layer was extracted with $\mathrm{Et}_{2} \mathrm{O}(2 \times 15 \mathrm{~mL})$, the organic layers were washed rapidly with ice water $(3 \times 10 \mathrm{~mL})$ and dried over $\mathrm{Na}_{2} \mathrm{SO}_{4}$. After filtration, $\mathrm{Et}_{2} \mathrm{O}$ was removed at $50{ }^{\circ} \mathrm{C}$ under argon and the residues were poured onto a mixture of pyridine (distilled, $16 \mathrm{~mL}, 0.20 \mathrm{~mol}, 1.0$ equiv) and $\alpha$-nitroso- $\beta$-naphtol $(0.2 \mathrm{~g}, 1 \mathrm{mmol}, 0.005$ equiv). After stirring $90 \mathrm{~min}$ at $23{ }^{\circ} \mathrm{C}$, the reaction mixture was slowly heated to $100{ }^{\circ} \mathrm{C}$ and the pressure lowered to 70 mbar, whereas crude 76 distilled to a flask cooled to $0{ }^{\circ} \mathrm{C}$ containing few $\alpha$-nitroso- $\beta$-naphtol. When the distillation was nearly finished, the temperature was increased to $125{ }^{\circ} \mathrm{C}$. Crude 76 was poured onto crushed ice $(20 \mathrm{~g})$ and concentrated $\mathrm{HCl}(37 \%, 20 \mathrm{~mL})$, the mixture was extracted with $\mathrm{Et}_{2} \mathrm{O}(3 \times 50 \mathrm{~mL})$, the combined organic layers were washed with $1 \% \mathrm{HCl}(2 \times 20 \mathrm{~mL})$ and $2 \% \mathrm{NH}_{3}$ $(2 \times 20 \mathrm{~mL})$ and dried over $\mathrm{Na}_{2} \mathrm{SO}_{4}$. After filtration, the solvent was removed under nitrogen $\left(p=700 \mathrm{mbar}, T=40{ }^{\circ} \mathrm{C}\right)$ and the residues were distilled over $\alpha$-nitroso- $\beta$-naphtol under nitrogen $\left(p=70 \mathrm{mbar}, b p=60-66^{\circ} \mathrm{C}\right)$ to afford 76 with $90 \%$ puritiy $(6.1 \mathrm{~g}$, $50 \mathrm{mmol}, 25 \%)$ contaminated with few paraldehyde. This mixture was kept at $-20{ }^{\circ} \mathrm{C}$ protected from light over $\alpha$-nitroso- $\beta$ naphtol and was used directly as such in the hydrohydrazination reaction. ${ }^{1} \mathrm{H} \mathrm{NMR}\left(\mathrm{CDCl}_{3}, 300 \mathrm{MHz}\right)^{8 \mathrm{~b}} \delta 7.21(\mathrm{~m}, 1 \mathrm{H}$, thiophene $\mathrm{H}), 7.02(\mathrm{~m}, 2 \mathrm{H}$, thiophene $\mathrm{H}), 6.87$ (ddd, $J=17.6,11.0,0.8 \mathrm{~Hz}, 1 \mathrm{H}$, vinyl H), $5.63(\mathrm{~d}, J=17.3 \mathrm{~Hz}, 1 \mathrm{H}$, vinyl H), $5.19(\mathrm{~d}, J=$ 11.3 Hz, 1H, vinyl H); ${ }^{13} \mathrm{C} \mathrm{NMR}\left(\mathrm{CDCl}_{3}, 75 \mathrm{MHz}\right) \delta 142.8,129.7,127.1,125.6,124.1,113.1$.

2-Vinyl-pyrrole (78)

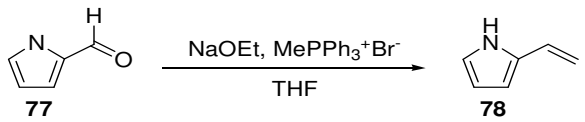

Following a slightly modified reported procedure, ${ }^{9}$ methyltriphenylphosphonium bromide $(9.0 \mathrm{~g}, 25 \mathrm{mmol}, 1.0$ equiv) was added to a suspension of NaOEt (1.8 g, $26 \mathrm{mmol}, 1.1$ equiv) in THF $(20 \mathrm{~mL})$ at $23{ }^{\circ} \mathrm{C}$ under argon. After $2.5 \mathrm{~h}$, pyrrole-2carbaldehyde (77) $(2.4 \mathrm{~g}, 25 \mathrm{mmol}, 1.0$ equiv) was added dropwise as a solution in THF (10 mL) over $15 \mathrm{~min}$. The yellow suspension was refluxed for $16 \mathrm{~h}$, cooled to $23{ }^{\circ} \mathrm{C}$, filtered and the solvents were removed under reduced pressure. The residues were suspended in $\mathrm{CH}_{2} \mathrm{Cl}_{2}$ and filtered. The solution was washed with $20 \% \mathrm{NaHSO}_{3}(25 \mathrm{~mL})$ and $10 \% \mathrm{Na}_{2} \mathrm{CO}_{3}(25 \mathrm{~mL})$, dried over $\mathrm{Na}_{2} \mathrm{SO}_{4}$ and the solvent was removed under reduced pressure under nitrogen. Distillation of the crude product over 4-tertbutyl catechol $\left(p=0.5 \mathrm{mbar}, b p=30-35^{\circ} \mathrm{C}\right)$ affords $78(0.62 \mathrm{~g}, 6.7 \mathrm{mmol}, 27 \%)$ as a colorless oil. Compound 78 was kept at -20 ${ }^{\circ} \mathrm{C}$ protected from light over 4-tert-butyl catechol. ${ }^{1} \mathrm{H}$ NMR $\left(\mathrm{CDCl}_{3}, 300 \mathrm{MHz}\right) \delta 8.26$ (br s, $\left.1 \mathrm{H}, \mathrm{NH}\right), 6.79(\mathrm{~d}, J=1.2 \mathrm{~Hz}, 1 \mathrm{H}$, pyrrole $\mathrm{H}), 6.64$ (ddm, $J=17.7,11.2,1 \mathrm{H}$, vinyl H), 6.35-6.28 (m, 2H, pyrrole H), $5.34(\mathrm{dm}, J=17.7 \mathrm{~Hz}, 1 \mathrm{H}$, vinyl H), $5.09(\mathrm{dm}$, $J=11.2 \mathrm{~Hz}, 1 \mathrm{H}$, vinyl $\mathrm{H}) ;{ }^{13} \mathrm{C} \mathrm{NMR}\left(\mathrm{CDCl}_{3}, 75 \mathrm{MHz}\right) \delta 130.6,126.9,118.7,109.2,108.2,107.9 ;$ IR $v 3400(\mathrm{~s}), 3090(\mathrm{w}), 3007$ (w), $2980(\mathrm{w}), 1634$ (s), $1545(\mathrm{w}), 1459$ (w), $1419(\mathrm{w}), 1390(\mathrm{w}), 1356(\mathrm{w}), 1298(\mathrm{w}), 1278(\mathrm{w}), 1231(\mathrm{w}), 1120(\mathrm{~m}), 1092(\mathrm{~m})$, $1035(\mathrm{~m}), 1020(\mathrm{~m}), 984(\mathrm{~m}), 948(\mathrm{w}), 883(\mathrm{~s}), 803(\mathrm{~m}), 731(\mathrm{~s}), 649(\mathrm{w}), 589(\mathrm{w}), 539(\mathrm{w})$.

1-Methyl-2-vinyl-1H-imidazole $(80)^{10}$

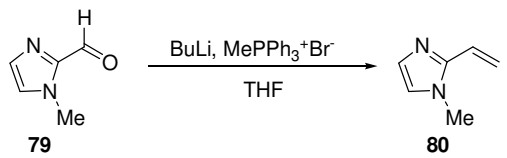

Following a reported procedure, ${ }^{10} \mathrm{BuLi}(1.6 \mathrm{M}$ in hexane, $4.4 \mathrm{~mL}, 7.0 \mathrm{mmol}, 1.1$ equiv) was added to a suspension of methyltriphenylphosphonium bromide $\left(2.3 \mathrm{~g}, 6.4 \mathrm{mmol}, 1.0\right.$ equiv) in THF $(15 \mathrm{~mL})$ at $0{ }^{\circ} \mathrm{C}$ under argon. The reaction mixture was warmed to $23{ }^{\circ} \mathrm{C}$ and stirred for $40 \mathrm{~min}$. A solution of $79(0.71 \mathrm{~g}, 6.4 \mathrm{mmol}, 1.0$ equiv) in THF (15 mL) was added dropwise, followed by THF ( $5 \mathrm{~mL}$ ). After stirring at $23{ }^{\circ} \mathrm{C}$ for $22 \mathrm{~h}$, the mixture was filtered, the filter cake was washed with $\mathrm{Et}_{2} \mathrm{O}$ and the solvent was removed. The crude product was purified by column chromatography (acetone: $\mathrm{CH}_{2} \mathrm{Cl}_{2} 7: 1$ ) followed by distillation to give $80(0.30 \mathrm{~g}, 2.8 \mathrm{mmol}, 44 \%)$ as a colorless liquid. ${ }^{1} \mathrm{H} \mathrm{NMR}\left(\mathrm{CDCl}_{3}, 300 \mathrm{MHz}\right) \delta 7.02(\mathrm{~s}, 1 \mathrm{H}$, imidazole $\mathrm{H}), 6.83(\mathrm{~s}, 1 \mathrm{H}$, imidazole $\mathrm{H}), 6.59$ (dd, $J=17.3,11.2,1 \mathrm{H}$, vinyl $\mathrm{H}), 6.14(\mathrm{dd}, J=17.3,1.5 \mathrm{~Hz}, 1 \mathrm{H}$, vinyl $\mathrm{H}), 5.40(\mathrm{dd}, J=11.2,1.5 \mathrm{~Hz}, 1 \mathrm{H}$, vinyl $\mathrm{H}), 3.65\left(\mathrm{~s}, 3 \mathrm{H}, \mathrm{CH}_{3}\right) ;{ }^{13} \mathrm{C} \mathrm{NMR}\left(\mathrm{CDCl}_{3}, 75 \mathrm{MHz}\right) \delta 145.2,128.3,122.5,121.3,117.8,32.8$;

\section{2-Vinyl-pyrazine $(\mathbf{8 3})^{11}$}




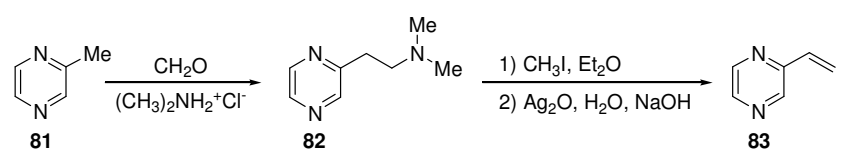

\section{Dimethyl-(2-pyrazin-2-yl-ethyl)-amine (82)}

Following a slightly modified reported procedure, ${ }^{11}$ formaldehyde $(37 \%, 7.2 \mathrm{~mL}, 96 \mathrm{mmol}, 1.7$ equiv) was added dropwise over $30 \mathrm{~min}$ to a refluxing $\left(135^{\circ} \mathrm{C}\right)$ mixture of 2-methyl-pyrazine $(\mathbf{8 1})(5.0 \mathrm{~mL}, 55 \mathrm{mmol}, 1.0$ equiv) and dimethylamine hydrochloride ( $4.7 \mathrm{~g}, 58 \mathrm{mmol}$. 1.1 equiv). After stirring $4 \mathrm{~h}$ at reflux, the reaction mixture was cooled to $23{ }^{\circ} \mathrm{C}$, water $(15 \mathrm{~mL})$ was added and the $\mathrm{pH}$ was adjusted to $>12$ with $\mathrm{NaOH}$ pellets. The reaction mixture was extracted with $\mathrm{CH}_{2} \mathrm{Cl}_{2}(3 \times 25 \mathrm{~mL})$, the combined organic layers were dried over $\mathrm{Na}_{2} \mathrm{SO}_{4}$ and the solvent was removed under reduced pressure. The crude mixture was purified by distillation $\left(p=0.5 \mathrm{mbar}, b p=55^{\circ} \mathrm{C}\right)$ to give $82(4.3 \mathrm{~g}, 29 \mathrm{mmol}, 52 \%)$ as a colorless oil. ${ }^{1} \mathrm{H} \mathrm{NMR}\left(\mathrm{CDCl}_{3}, 300 \mathrm{MHz}\right) \delta 8.31-$ $8.19(\mathrm{~m}, 3 \mathrm{H}$, pyrazine $\mathrm{H}), 2.81-2.75\left(\mathrm{~m}, 2 \mathrm{H}, \mathrm{CH}_{2}\right), 2.55-2.48\left(\mathrm{~m}, 2 \mathrm{H}, \mathrm{CH}_{2}\right), 2.10\left(\mathrm{~s}, 3 \mathrm{H}, \mathrm{CH}_{3}\right), 2.09\left(\mathrm{~s}, 3 \mathrm{H}, \mathrm{CH}_{3}\right) ;{ }^{13} \mathrm{C} \mathrm{NMR}$ $\left(\mathrm{CDCl}_{3}, 75 \mathrm{MHz}\right) \delta 155.6,144.4,143.5,141.8,58.4,45.1,33.4$; IR $v 3054(\mathrm{w}), 2972(\mathrm{~m}), 2943(\mathrm{~s}), 2859(\mathrm{~m}), 2818(\mathrm{~s}), 2768(\mathrm{~s})$, 1578 (w), 1526 (w), 1463 (s), 1403 (s), $1374(w), 1308$ (w), 1266 (w), $1232(w), 1155$ (w), $1123(\mathrm{~m}), 1098$ (w), 1054 (s), 1016 (m), $920(\mathrm{w}), 879(\mathrm{w}), 837(\mathrm{~m}), 778(\mathrm{w}), 656(\mathrm{w})$.

\section{2-Vinyl-pyrazine (83)}

$\mathrm{CH}_{3} \mathrm{I}\left(1.8 \mathrm{~mL}, 29 \mathrm{mmol}, 1.1\right.$ equiv) was added dropwise to a solution of 82 (4.2 g, $28 \mathrm{mmol}, 1.0$ equiv) in $\mathrm{Et}_{2} \mathrm{O}(28 \mathrm{~mL})$, whereas the ammonium salt precipated. After $18 \mathrm{~h}$, water $(20 \mathrm{~mL})$ and $\mathrm{Et}_{2} \mathrm{O}(20 \mathrm{~mL})$ were added, the layers were separated and the organic layer was extracted with water $(8 \mathrm{~mL})$. The combined water layers were cooled to $0{ }^{\circ} \mathrm{C}$ and $\mathrm{Ag}_{2} \mathrm{O}(13 \mathrm{~g}, 56 \mathrm{mmol}, 2.0$ equiv) was added. After $3 \mathrm{~h}$, the reaction mixture was filtered over Celite, the Celite was washed with water $(20 \mathrm{~mL})$ and $\mathrm{NaOH}$ $(1.2 \mathrm{~g}, 30 \mathrm{mmol}, 1.1$ equiv) was added. The solution was heated to reflux over $30 \mathrm{~min}$ and refluxed for $1 \mathrm{~h}$. After the evolution of trimethyl amine was finished, the reaction mixture was cooled to $23{ }^{\circ} \mathrm{C}$ and extracted with $\mathrm{CH}_{2} \mathrm{Cl}_{2}(3 \times 50 \mathrm{~mL})$. The combined organic layers were dried over $\mathrm{Na}_{2} \mathrm{SO}_{4}$ and the solvent was removed under reduced pressure under nitrogen. The crude product was purified by distillation under nitrogen over 4-tert-butyl catechol $\left(p=25 \mathrm{mbar}, b p=60{ }^{\circ} \mathrm{C}\right)$ to give $\mathbf{8 3}(1.7 \mathrm{~g}, 16 \mathrm{mmol}, 58 \%)$ as a colorless oil. ${ }^{1} \mathrm{H} \mathrm{NMR}\left(\mathrm{CDCl}_{3}, 300 \mathrm{MHz}\right) \delta 8.45(\mathrm{~d}, J=1.2 \mathrm{~Hz}, 1 \mathrm{H}$, pyrazine $\mathrm{H}), 8.37(\mathrm{dd}, J=2.5,1.2 \mathrm{~Hz}, 1 \mathrm{H}, \mathrm{pyrazine} \mathrm{H})$, 8.29-8.27 (m, $1 \mathrm{H}$, pyrazine $\mathrm{H}), 6.68(\mathrm{ddm}, J=17.4,10.9,1 \mathrm{H}$, vinyl $\mathrm{H}), 6.22(\mathrm{dm}, J=17.4 \mathrm{~Hz}, 1 \mathrm{H}$, vinyl $\mathrm{H}), 5.47(\mathrm{dm}, J=10.9$ $\mathrm{Hz}, 1 \mathrm{H}$, vinyl H); ${ }^{13} \mathrm{C} \mathrm{NMR}\left(\mathrm{CDCl}_{3}, 75 \mathrm{MHz}\right) \delta 150.7,143.9,143.0,142.8,133.2,120.5 ; \mathrm{IR} v 3064(\mathrm{~m}), 3028(\mathrm{w}), 1874(\mathrm{w}), 1633$ (w), $1567(\mathrm{w}), 1520$ (m), 1476 (s), $1416(\mathrm{~s}), 1382(\mathrm{~m}), 1308$ (m), 1231 (w), 1153 (s), 1059 (m), 1030 (s), 1013 (s), 989 (s), 936 (s), $854(\mathrm{~s}), 770(\mathrm{w}), 733(\mathrm{w}), 661(\mathrm{w}), 636(\mathrm{w}), 562(\mathrm{w})$.

\section{2-Vinyl-1H-indole (86)}
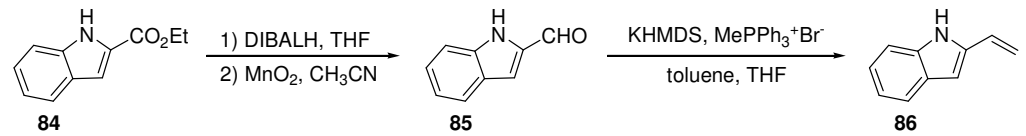

\section{$1 H$-Indole-2-carbaldehyde (85)}

Following a reported procedure, ${ }^{12}$ a solution of DIBALH $(4.3 \mathrm{~mL}, 26 \mathrm{mmol}, 2.4$ equiv) in THF (60 mL) was added dropwise to a solution of indole ester $84\left(2.0 \mathrm{~g}, 11 \mathrm{mmol}, 1.0\right.$ equiv) in THF $(70 \mathrm{~mL})$ at $-78{ }^{\circ} \mathrm{C}$ under argon. The solution was let to warm up to $23{ }^{\circ} \mathrm{C}$ over $14 \mathrm{~h}$ and sat. $\mathrm{Na}^{+} / \mathrm{K}^{+}$tartrate solution $(120 \mathrm{~mL})$ was added at $0{ }^{\circ} \mathrm{C}$ over $30 \mathrm{~min}$. After stirring for $6 \mathrm{~h}$, AcOEt $(100$ $\mathrm{ml})$ was added, the layers were separated, the water layer was extracted with AcOEt $(2 \times 100 \mathrm{~mL})$, the combined organic layers were dried over $\mathrm{Na}_{2} \mathrm{SO}_{4}$ and the solvent was removed under reduced pressure.

The residues were dissolved in $\mathrm{CH}_{3} \mathrm{CN}(60 \mathrm{~mL})$ and $\mathrm{MnO}_{2}(5.0 \mathrm{~g})$ was added under argon. After complete oxidation by TLC (AcOEt/hexane 1:2) $\left(18 \mathrm{~h}\right.$ ), the reaction mixture was filtered over Celite, the Celite was washed with $\mathrm{CH}_{3} \mathrm{CN}(30 \mathrm{~mL})$ and the solvent was removed under reduced pressure. Recrystallization from AcOEt/hexane 1:4 gives aldehyde 85 (1.21 g, $8.37 \mathrm{mmol}$, $79 \%$ ) as slightly brown crystals. Mp (AcOEt/hexane 1:4) $130-132{ }^{\circ} \mathrm{C}\left(\mathrm{Lit} . .^{12} 141-143{ }^{\circ} \mathrm{C}\right) ;{ }^{1} \mathrm{H} \mathrm{NMR}(\mathrm{CDCl} 3,300 \mathrm{MHz}) \delta 9.86(\mathrm{~s}$, $1 \mathrm{H}$, aldehyde $\mathrm{H}), 9.44$ (br s, $1 \mathrm{H}, \mathrm{NH}), 7.75(\mathrm{~d}, J=8.1 \mathrm{~Hz}, 1 \mathrm{H}$, indole $\mathrm{H}), 7.50-7.16(\mathrm{~m}, 4 \mathrm{H}$, indole $\mathrm{H}) ;{ }^{13} \mathrm{C} \mathrm{NMR}\left(\mathrm{CDCl} \mathrm{N}_{3}, 75\right.$ MHz) $\delta 182.2,138.2,135.8,127.2,127.1,123.2,121.0,115.2,112.6$; IR (KBr) v $3179(\mathrm{~m}), 3057(\mathrm{w}), 2853(\mathrm{w}), 1683(\mathrm{w}), 1651$ (m), $1620(\mathrm{w}), 1528(\mathrm{~m}), 1448(\mathrm{w}), 1429(\mathrm{w}), 1363(\mathrm{w}), 1341(\mathrm{~s}), 1254(\mathrm{~m}), 1231(\mathrm{~m}), 1128(\mathrm{~s}), 1005(\mathrm{w}), 996(\mathrm{w}), 985(\mathrm{w}), 936$ (w), $897(\mathrm{w}), 856(\mathrm{w}), 822(\mathrm{~s}), 742(\mathrm{~s}), 611(\mathrm{w}), 582(\mathrm{~m}), 466(\mathrm{~m}), 444(\mathrm{~m})$.

\section{2-Vinyl-1H-indole (86)}

A solution of KHMDS (1.2 g, $6.0 \mathrm{mmol}, 1.3 \mathrm{equiv})$ in toluene $(12 \mathrm{~mL})$ was added to a suspension of methyltriphenylphosphonium bromide $\left(2.5 \mathrm{~g}, 7.0 \mathrm{mmol}, 1.5\right.$ equiv) in THF $(34 \mathrm{~mL})$ at $23{ }^{\circ} \mathrm{C}$ under argon. After $30 \mathrm{~min}$, the reaction was transferred dropwise via canula under argon to a solution of aldehyde $\mathbf{8 5}(0.67 \mathrm{~g}, 4.6 \mathrm{mmol}, 1 \mathrm{equiv})$ in THF (23 $\mathrm{mL})$. After stirring at $23{ }^{\circ} \mathrm{C}$ for $3 \mathrm{~h}$, the reaction mixture was poured onto $\mathrm{Et}_{2} \mathrm{O}(50 \mathrm{~mL})$ and water $(50 \mathrm{~mL})$, the layers were separated, the water layer was extracted with $\mathrm{Et}_{2} \mathrm{O}(2 \times 50 \mathrm{~mL})$, the combined organic layers were dried over $\mathrm{Na}_{2} \mathrm{SO}_{4}$ and the solvent was removed under reduced pressure. The crude product was purified by column chromatography (AcOEt/hexane 1:20) to give 86 (0.60 g, $4.2 \mathrm{mmol}, 90 \%)$ as a colorless solid. $\mathrm{Mp} 83-85{ }^{\circ} \mathrm{C}\left(\mathrm{Lit} .:^{12} 85-86{ }^{\circ} \mathrm{C}\right) ;{ }^{1} \mathrm{H} \mathrm{NMR}\left(\mathrm{CDCl}_{3}, 300 \mathrm{MHz}\right) \delta 8.14(\mathrm{br} \mathrm{s}$, $1 \mathrm{H}, \mathrm{NH}), 7.58(\mathrm{~d}, J=7.8 \mathrm{~Hz}, 1 \mathrm{H}$, indole $\mathrm{H}), 7.34(\mathrm{dd}, J=8.1,0.9 \mathrm{~Hz}, 1 \mathrm{H}$, indole $\mathrm{H}), 7.20$ (ddd, $J=8.1,7.2,1.2 \mathrm{~Hz}, 1 \mathrm{H}$, indole $\mathrm{H}), 7.10(\mathrm{ddd}, J=8.1,7.2,1.3 \mathrm{~Hz}, 1 \mathrm{H}$, indole $\mathrm{H}), 6.75(\mathrm{dd}, J=17.7,11.2 \mathrm{~Hz}, 1 \mathrm{H}$, vinyl H), $6.52(\mathrm{~d}, J=2.2 \mathrm{hz}, 1 \mathrm{H}$, indole H), $5.56(\mathrm{~d}, J=17.7 \mathrm{~Hz}, 1 \mathrm{H}$, vinyl $\mathrm{H}), 5.27(\mathrm{~d}, J=11.2 \mathrm{~Hz}, 1 \mathrm{H}$, vinyl $\mathrm{H}) ;{ }^{13} \mathrm{C} \mathrm{NMR}\left(\mathrm{CDCl}_{3}, 75 \mathrm{MHz}\right) \delta 136.4,136.1,128.5,127.4$, 
122.7, 120.6, 120.0, 112.0, 110.6, 103.0; IR v 3405 (s), 3057 (w), $2922(w), 1892(w), 1782(w), 1639(w), 1612(w), 1526(w)$, $1454(\mathrm{~m}), 1424(\mathrm{~m}), 1400(\mathrm{~m}), 1340(\mathrm{~m}), 1292(\mathrm{~m}), 1234(\mathrm{w}), 1154(\mathrm{w}), 1132(\mathrm{w}), 1037(\mathrm{w}), 1010(\mathrm{w}), 984(\mathrm{~m}), 930(\mathrm{w}), 907$ (s), $797(\mathrm{~s}), 741(\mathrm{~s}), 649(\mathrm{w}), 609(\mathrm{w}), 578(\mathrm{w}), 567(\mathrm{w}), 466(\mathrm{w})$.

(4-Vinyl-phenyl)-carbamic acid 9,9a-dihydro-4aH-fluoren-9-ylmethyl ester (88)

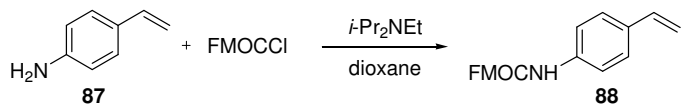

Fluoranthenylmethoxycarbonyl chloride (1.2 g, $4.8 \mathrm{mmol}, 1.2$ equiv) and Hünig's base ( $0.84 \mathrm{~mL}, 4.8 \mathrm{mmol}, 1.2$ equiv) were added to a solution of 4-vinylpyridine $(\mathbf{8 7})\left(90 \%, 0.53 \mathrm{~g}, 4.0 \mathrm{mmol}, 1.0\right.$ equiv) in dioxane $(8 \mathrm{~mL})$ at $0{ }^{\circ} \mathrm{C}$ under argon. The reaction mixture was stirred at $0{ }^{\circ} \mathrm{C}$ for $30 \mathrm{~min}$ and warmed to $23{ }^{\circ} \mathrm{C}$. After $2 \mathrm{~h}, \mathrm{CH}_{2} \mathrm{Cl}_{2}(50 \mathrm{~mL})$, sat. $\mathrm{NH}_{4} \mathrm{Cl}(20 \mathrm{~mL})$ and brine $(20 \mathrm{~mL})$ were added, the organic layer was separated and the water layer was extracted with $\mathrm{CH}_{2} \mathrm{Cl}_{2}(2 \mathrm{x} 40 \mathrm{~mL})$. The combined organic layers were concentrated under reduced pressure and the isolated product was purified via precipitation with hexane from a solution in $\mathrm{CH}_{2} \mathrm{Cl}_{2}$ to afford $\mathbf{8 8}(1.28 \mathrm{~g}, 3.75 \mathrm{mmol}, 94 \%)$ as a colorless solid. Mp 194-197 ${ }^{\circ} \mathrm{C} ;{ }^{1} \mathrm{H} \mathrm{NMR}(\mathrm{CDCl} 3,300 \mathrm{MHz}) \delta$ $7.79(\mathrm{~d}, J=7.5 \mathrm{~Hz}, 2 \mathrm{H}, \mathrm{Ar} \mathrm{H}), 7.62(\mathrm{~d}, J=7.5 \mathrm{~Hz}, 2 \mathrm{H}, \mathrm{Ar} \mathrm{H}), 7.42(\mathrm{t}, J=7.5 \mathrm{~Hz}, 2 \mathrm{H}, \mathrm{Ar} \mathrm{H}), 7.35-7.30(\mathrm{~m}, 6 \mathrm{H}, \mathrm{Ar} \mathrm{H}), 6.67(\mathrm{dd}, J$ $=17.4,10.9 \mathrm{~Hz}, 1 \mathrm{H}$, vinyl H), 6.65 (br s, $1 \mathrm{H}, \mathrm{NH}), 5.67(\mathrm{dd}, J=17.7,0.9,1 \mathrm{H}$, vinyl H), $5.19(\mathrm{dd}, J=10.9,0.6 \mathrm{~Hz}, 1 \mathrm{H}$, vinyl H), $4.55\left(\mathrm{~d}, J=6.5 \mathrm{~Hz}, 2 \mathrm{H}, \mathrm{CH}_{2} \mathrm{O}\right), 4.28\left(\mathrm{t}, J=6.5 \mathrm{~Hz}, 1 \mathrm{H}, \mathrm{CHCH}_{2} \mathrm{O}\right) ;{ }^{13} \mathrm{C} \mathrm{NMR}\left(\mathrm{CDCl}_{3}, 75 \mathrm{MHz}\right) \delta 153.2,143.7,141.4,137.2$, 136.0, 133.1, 127.8, 127.1, 126.9, 124.9, 120.0, 118.7, 112.8, 66.8, 47.1; IR v 3308 (w), $2361(\mathrm{w}), 1698$ (s), 1609 (w), $1586(\mathrm{w})$, 1525 (s), 1450 (w), 1414 (w), 1404 (w), 1317 (w), 1236 (m), $1225(\mathrm{~m}), 1089(\mathrm{w}), 1053(\mathrm{w}), 1013(\mathrm{w}), 987(\mathrm{w}), 905(\mathrm{w}), 836(\mathrm{w})$, 759 (w), 737 (m), 668 (w); HRMS (MALDI) calcd for $\mathrm{C}_{23} \mathrm{H}_{19} \mathrm{NO}_{2} \mathrm{Na}^{+}$(M+Na): 364.1308, found 364.1305; Anal. calcd for $\mathrm{C}_{23} \mathrm{H}_{19} \mathrm{NO}_{2}$ : C, 80.92; H, 5.61; N, 4.10. Found: C, 80.74; H, 5.59; N, 4.05.

1-(Toluene-4-sulfonyl)-2-vinyl-1H-pyrrole (90)
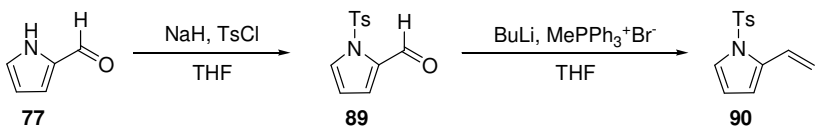

1-(Toluene-4-sulfonyl)-1 $H$-pyrrole-2-carbaldehyde $(89)^{13}$

Following a reported procedure, ${ }^{14}$ a solution of pyrrole-2-carbaldehyde (77) $(1.0 \mathrm{~g}, 11 \mathrm{mmol}, 1$ equiv) in THF (10 mL) was added over $10 \mathrm{~min}$ to a suspension of $\mathrm{NaH}\left(0.30 \mathrm{~g}, 13 \mathrm{mmol}, 1.2\right.$ equiv) in THF $(60 \mathrm{~mL})$ at $23{ }^{\circ} \mathrm{C}$ under argon. After $1 \mathrm{~h}$, tosyl chloride ( $2.2 \mathrm{~g}, 12 \mathrm{mmol}, 1.1$ equiv) was added. After stirring for $13 \mathrm{~h}$ at $23{ }^{\circ} \mathrm{C}$, the reaction mixture was quenched with sat. $\mathrm{NH}_{4} \mathrm{Cl}(10 \mathrm{~mL})$, diluted with water $(200 \mathrm{~mL})$ and extracted with $\mathrm{Et}_{2} \mathrm{O}(3 \times 100 \mathrm{~mL})$. The combined organic layer were washed with brine $(50 \mathrm{~mL})$, dried over $\mathrm{Na}_{2} \mathrm{SO}_{4}$ and the solvent was removed under reduced pressure. The crude product was purified by column chromatography (AcOEt/hexane 1:10) to give $89(2.5 \mathrm{~g}, 9.9 \mathrm{mmol}, 94 \%)$ as a colorless solid. Mp $92-94{ }^{\circ} \mathrm{C}\left(\mathrm{Lit} . \mathrm{:}^{13} 94{ }^{\circ} \mathrm{C}\right)$; ${ }^{1} \mathrm{H} \mathrm{NMR}\left(\mathrm{CDCl}_{3}, 300 \mathrm{MHz}\right) \delta 9.96(\mathrm{~s}, 1 \mathrm{H}$, aldehyde $\mathrm{H}), 7.79(\mathrm{~d}, J=8.4 \mathrm{~Hz}, 2 \mathrm{H}$, phenyl H), $7.61(\mathrm{~m}, 1 \mathrm{H}$, pyrrole $\mathrm{H}), 7.31(\mathrm{~d}, J=$ $8.1 \mathrm{~Hz}, 2 \mathrm{H}$, phenyl H), $7.14(\mathrm{~m}, 1 \mathrm{H}$, pyrrole $\mathrm{H}), 6.40(\mathrm{~m}, 1 \mathrm{H}$, pyrrole $\mathrm{H}), 2.40\left(\mathrm{~s}, 3 \mathrm{H}, \mathrm{CH}_{3}\right) ;{ }^{13} \mathrm{C} \mathrm{NMR}\left(\mathrm{CDCl}{ }_{3}, 75 \mathrm{MHz}\right) \delta 178.7$, 145.8, 135.0, 133.3, 130.0, 129.3, 127.3, 124.3, 112.3, 21.7; IR v 3126 (w), 3066 (w), 2895 (w), 2840 (w), 1672 (s), 1595 (w), 1538 (w), 1494 (w), 1448 (m), 1423 (s), 1409 (m), 1374 (s), 1332 (w), 1308 (w), 1296 (w), 1249 (m), 1234 (w), 1215 (w), 1192 (s), 1175 (s), 1158 (s), 1141 (s), 1090 (m), 1055 (m), 1013 (w), 880 (w), 842 (w), 814 (m), 775 (m), 757 (m), 702 (w), 670 (s), $634(\mathrm{w}), 587$ (s), $563(\mathrm{~m}), 539(\mathrm{~m}), 489(\mathrm{w}), 449(\mathrm{w}), 424(\mathrm{w})$.

\section{1-(Toluene-4-sulfonyl)-2-vinyl-1H-pyrrole $(90)^{15}$}

Following a reported procedure, ${ }^{14} \mathrm{BuLi}(1.6 \mathrm{M}$ in hexane, $5.5 \mathrm{~mL}, 8.8 \mathrm{mmol}, 1.1$ equiv) was added to a suspension of methyltriphenylphosphonium bromide $\left(3.4 \mathrm{~g}, 9.5 \mathrm{mmol}, 1.2\right.$ equiv) in THF $(70 \mathrm{~mL})$ at $0{ }^{\circ} \mathrm{C}$ under argon. After stirring for $2 \mathrm{~h}$, the reaction mixture was cooled to $-78{ }^{\circ} \mathrm{C}$ and a solution of $\mathbf{8 9}(2.0 \mathrm{~g}, 8.0 \mathrm{mmol}, 1.0$ equiv) in THF $(10 \mathrm{~mL})$ was added dropwise. The reaction was let to warm up to $23{ }^{\circ} \mathrm{C}$ over $12 \mathrm{~h}$, quenched with water $(150 \mathrm{~mL})$ and extracted with $\mathrm{Et}_{2} \mathrm{O}(3 \times 100 \mathrm{~mL})$. The combined organic layer were washed with water $(50 \mathrm{~mL})$ and brine $(50 \mathrm{~mL})$, dried over $\mathrm{Na}_{2} \mathrm{SO}_{4}$ and the solvent was removed under reduced pressure. The crude product was purified by column chromatography (AcOEt/hexane 1:15) to give 90 (1.8 g, 7.3 mmol, 91\%) as a colorless solid. Mp 66-67 ${ }^{\circ} \mathrm{C} ;{ }^{1} \mathrm{H} \mathrm{NMR}\left(\mathrm{CDCl}_{3}, 300 \mathrm{MHz}\right) \delta 7.69(\mathrm{~d}, J=8.4 \mathrm{~Hz}, 2 \mathrm{H}, \mathrm{phenyl} \mathrm{H}), 7.31-7.28(\mathrm{~m}$, $1 \mathrm{H}$, pyrrole $\mathrm{H}), 7.27(\mathrm{~d}, J=8.1 \mathrm{~Hz}, 2 \mathrm{H}$, phenyl H), $7.10(\mathrm{dd}, J=17.4,11.2 \mathrm{~Hz}, 1 \mathrm{H}$, vinyl H), $6.44(\mathrm{~m}, 1 \mathrm{H}$, pyrrole $\mathrm{H}), 6.24(\mathrm{~m}$, $1 \mathrm{H}$, pyrrole $\mathrm{H}), 5.48(\mathrm{dd}, J=17.4,1.2 \mathrm{~Hz}, 1 \mathrm{H}$, vinyl $\mathrm{H}), 5.15\left(\mathrm{dd}, J=11.2,1.5 \mathrm{~Hz}, 1 \mathrm{H}\right.$, vinyl H), $2.39\left(\mathrm{~s}, 3 \mathrm{H}, \mathrm{CH}_{3}\right) ;{ }^{13} \mathrm{C} \mathrm{NMR}$ $\left(\mathrm{CDCl}_{3}, 75 \mathrm{MHz}\right) \delta 144.8,135.8,133.8,129.7,126.7,125.3,123.0,114.9,112.1,111.6,21.6$; IR $v 3150(\mathrm{w}), 2926(\mathrm{w}), 1596(\mathrm{w})$, $1494(\mathrm{w}), 1465$ (w), 1421 (w), 1368 (s), 1309 (w), 1294 (w), 1244 (w), 1190 (s), 1179 (s), 1154 (s), 1129 (m), 1091 (m), 1060 (w), 1039 (w), 1018 (w), 1000 (w), 910 (w), 874 (w), 813 (m), 726 (m), 704 (m), 690 (m), 659 (m), 586 (s), 554 (w), 535 (w), 489 (w).

2-Vinyl-pyrrole-1-carboxylic acid tert-butyl ester $(92)^{14}$

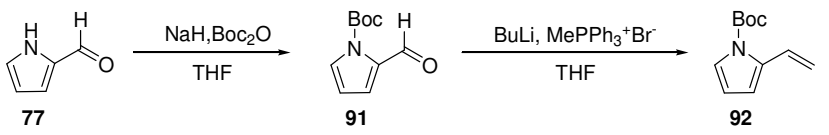

2-Formyl-pyrrole-1-carboxylic acid tert-butyl ester $(91)^{14}$ 
Following a reported procedure, ${ }^{14}$ a solution of pyrrole-2-carbaldehyde (77) $(1.0 \mathrm{~g}, 11 \mathrm{mmol}, 1.0$ equiv) in THF (10 mL) was added over $10 \mathrm{~min}$ to a suspension of $\mathrm{NaH}\left(0.30 \mathrm{~g}, 13 \mathrm{mmol}, 1.2\right.$ equiv) in THF $(60 \mathrm{~mL})$ at $23{ }^{\circ} \mathrm{C}$ under argon. After $1 \mathrm{~h}$, Boc anhydride ( $2.5 \mathrm{~g}, 12 \mathrm{mmol}, 1.1$ equiv) was added. After stirring for $13 \mathrm{~h}$ at $23{ }^{\circ} \mathrm{C}$, the reaction mixture was quenched with sat. $\mathrm{NH}_{4} \mathrm{Cl}(10 \mathrm{~mL})$, diluted with water $(200 \mathrm{~mL})$ and extracted with $\mathrm{Et}_{2} \mathrm{O}(3 \times 100 \mathrm{~mL})$. The combined organic layer were washed with brine $(50 \mathrm{~mL})$, dried over $\mathrm{Na}_{2} \mathrm{SO}_{4}$ and the solvent was removed under reduced pressure. The crude product was purified by column chromatography (AcOEt/hexane 1:30) to give $91(2.0 \mathrm{~g}, 10 \mathrm{mmol}, 97 \%)$ as an oil. ${ }^{1} \mathrm{H} \mathrm{NMR}\left(\mathrm{CDCl} \mathrm{N}_{3}, 300 \mathrm{MHz}\right) \delta 10.29(\mathrm{~s}$, $1 \mathrm{H}$, aldehyde $\mathrm{H}), 7.41(\mathrm{~m}, 1 \mathrm{H}$, pyrrole $\mathrm{H}), 7.14(\mathrm{~m}, 1 \mathrm{H}$, pyrrole $\mathrm{H}), 6.24(\mathrm{~m}, 1 \mathrm{H}$, pyrrole $\mathrm{H}), 1.61\left(\mathrm{~s}, 9 \mathrm{H}, \mathrm{CH}_{3}\right) ;{ }^{13} \mathrm{C} \mathrm{NMR}\left(\mathrm{CDCl}_{3}\right.$, $75 \mathrm{MHz}) \delta 182.0,148.1,134.5,127.1,120.9,111.5,85.7,27.9$. IR v 3150 (w), $2982(\mathrm{~m}), 2936(\mathrm{w}), 2906$ (w), 1749 (s), 1668 (s), 1544 (s), 1477 (w), 1442 (s), 1390 (m), 1372 (s), 1339 (s), 1300 (s), 1251 (s), 1166 (s), 1126 (s), 1066 (s), 1016 (w), 894 (m), 846 (s), $771(\mathrm{~m}), 749(\mathrm{~s}), 596(\mathrm{w}), 486(\mathrm{w})$.

\section{2-Vinyl-pyrrole-1-carboxylic acid tert-butyl ester (92)}

Following a reported procedure, ${ }^{14} \mathrm{BuLi}(1.6 \mathrm{M}$ in hexane, $5.5 \mathrm{~mL}, 8.8 \mathrm{mmol}, 1.1$ equiv) was added to a suspension of methyltriphenylphosphonium bromide $\left(3.4 \mathrm{~g}, 9.5 \mathrm{mmol}, 1.2\right.$ equiv) in THF $(70 \mathrm{~mL})$ at $0{ }^{\circ} \mathrm{C}$ under argon. After stirring for $2 \mathrm{~h}$, the reaction mixture was cooled to $-78{ }^{\circ} \mathrm{C}$ and a solution of $91(1.6 \mathrm{~g}, 8.0 \mathrm{mmol}, 1.0$ equiv) in THF $(10 \mathrm{~mL})$ was added dropwise. The reaction was let to warm up to $23{ }^{\circ} \mathrm{C}$ over $12 \mathrm{~h}$, quenched with water $(150 \mathrm{~mL})$ and extracted with $\mathrm{Et}_{2} \mathrm{O}(3 \times 100 \mathrm{~mL})$. The combined organic layer were washed with water $(50 \mathrm{~mL})$ and brine $(50 \mathrm{~mL})$, dried over $\mathrm{Na}_{2} \mathrm{SO}_{4}$ and the solvent was removed under reduced pressure. The crude product was purified by column chromatography (AcOEt/hexane 1:30) to give 92 (1.22 $\mathrm{g}, 6.33$ mmol, $79 \%)$ as a yellow oil. ${ }^{1} \mathrm{H}$ NMR $\left(\mathrm{CDCl}_{3}, 300 \mathrm{MHz}\right) \delta 7.25(\mathrm{~m}, 1 \mathrm{H}$, pyrrole $\mathrm{H}), 7.23(\mathrm{dd}, J=17.4,11.2 \mathrm{~Hz}, 1 \mathrm{H}$, vinyl H), $6.43(\mathrm{~m}, 1 \mathrm{H}$, pyrrole $\mathrm{H}), 6.14(\mathrm{~m}, 1 \mathrm{H}$, pyrrole $\mathrm{H}), 5.53(\mathrm{dd}, J=17.4,1.6 \mathrm{~Hz}, 1 \mathrm{H}$, vinyl $\mathrm{H}), 5.12(\mathrm{dd}, J=11.2,1.6 \mathrm{~Hz}, 1 \mathrm{H}$, vinyl $\mathrm{H}), 1.61\left(\mathrm{~s}, 9 \mathrm{H}, \mathrm{CH}_{3}\right) ;{ }^{13} \mathrm{C} \mathrm{NMR}\left(\mathrm{CDCl}_{3}, 75 \mathrm{MHz}\right) \delta 149.2,134.3,127.9,121.7,113.1,110.6,110.6,83.7,28.0 ; \mathrm{IR} v 3160(\mathrm{w})$, $3093(\mathrm{w}), 2980(\mathrm{~m}), 2935$ (w), $2874(\mathrm{w}), 1743(\mathrm{~s}), 1618(\mathrm{w}), 1557(\mathrm{w}), 1477(\mathrm{~m}), 1458(\mathrm{w}), 1423(\mathrm{~m}), 1396(\mathrm{~m}), 1371(\mathrm{~s}), 1336$ (s), $1308(\mathrm{~s}), 1286(\mathrm{~m}), 1245(\mathrm{~m}), 1168(\mathrm{~s}), 1124(\mathrm{~s}), 1066(\mathrm{~m}), 1044(\mathrm{w}), 1013(\mathrm{w}), 985(\mathrm{w}), 902(\mathrm{w}), 884(\mathrm{w}), 851(\mathrm{w}), 813(\mathrm{w})$, $772(\mathrm{w}), 729(\mathrm{~m}), 701(\mathrm{w}), 672(\mathrm{w}), 595(\mathrm{w}), 480(\mathrm{w}), 460(\mathrm{w})$.

\section{1-(Toluene-4-sulfonyl)-2-vinyl-1H-indole $(95)^{16}$}
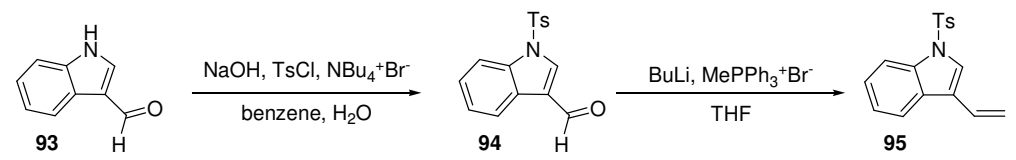

\section{1-(Toluene-4-sulfonyl)-1H-indole-2-carbaldehyde $(\mathbf{9 4})^{17}$}

Following a reported procedure, ${ }^{18}$ aqueous $30 \% \mathrm{NaOH}(25 \mathrm{~mL})$, tosyl chloride $(1.4 \mathrm{~g}, 7.2 \mathrm{mmol}, 1.1$ equiv) and tetrabutylammonium bromide $(0.22 \mathrm{~g}, 0.69 \mathrm{mmol}, 0.10$ equiv) were added to a suspension of indole aldehyde 93 ( $1.0 \mathrm{~g}, 6.9 \mathrm{mmol}$, 1.0 equiv) in benzene $(25 \mathrm{~mL})$. After stirring vigorously for $30 \mathrm{~min}$, the layers were separated and the water layer was extracted with benzene $(10 \mathrm{~mL})$. The combined organic layers were dried over $\mathrm{Na}_{2} \mathrm{SO}_{4}$ and the solvent was removed under reduced pressure. The crude product was purified by recrystallization from $\mathrm{CH}_{2} \mathrm{Cl}_{2}$ /hexane to afford aldehyde 94 (1.44 $\mathrm{g}, 4.82 \mathrm{mmol}$, $70 \%)$ as a colorless solid. $\mathrm{Mp}\left(\mathrm{CH}_{2} \mathrm{Cl}_{2} /\right.$ hexane) $146-147{ }^{\circ} \mathrm{C}\left(\mathrm{Lit} .:^{18} 147-149{ }^{\circ} \mathrm{C}\right) ;{ }^{1} \mathrm{H} \mathrm{NMR}\left(\mathrm{CDCl}_{3}, 300 \mathrm{MHz}\right) \delta 10.09(\mathrm{~s}, 1 \mathrm{H}$, aldehyde $\mathrm{H}), 8.25(\mathrm{dm}, J=7.2 \mathrm{~Hz}, 1 \mathrm{H}$, indole $\mathrm{H}), 8.23(\mathrm{~s}, 1 \mathrm{H}$, indole $\mathrm{H}), 7.95(\mathrm{~d}, J=8.4 \mathrm{~Hz}, 1 \mathrm{H}$, indole $\mathrm{H}), 7.85(\mathrm{~d}, \mathrm{~J}=8.4 \mathrm{~Hz}$, $2 \mathrm{H}$, phenyl H), 7.44-7.31 (m, 2H, indole $\mathrm{H}), 7.30\left(\mathrm{~d}, J=8.1 \mathrm{~Hz}, 2 \mathrm{H}\right.$, phenyl H), $2.38\left(\mathrm{~s}, 3 \mathrm{H}, \mathrm{CH}_{3}\right) ;{ }^{13} \mathrm{C} \mathrm{NMR}\left(\mathrm{CDCl}_{3}, 75 \mathrm{MHz}\right) \delta$ 185.1, 146.0, 136.1, 135.0, 134.1, 130.1, 127.1, 126.1, 124.9, 122.4, 122.2, 113.1, 21.7; IR v 3128 (w), 3064 (w), 2922 (w), 2824 (w), 2740 (w), 1920 (w), 1807 (w), 1679 (s), 1596 (w), 1542 (m), 1493 (w), 1480 (w), 1446 (s), 1380 (s), 1334 (w), 1307 (w), 1293 (w), 1277 (m), 1233 (m), 1214 (w), 1190 (s), 1178 (s), 1142 (m), 1127 (s), 1100 (s), 1085 (m), 1039 (w), 1018 (w), 971 (s), $911(\mathrm{w}), 813(\mathrm{w}), 781(\mathrm{~m}), 749(\mathrm{~s}), 710(\mathrm{~m}), 662(\mathrm{~s}), 589(\mathrm{~s}), 572(\mathrm{~s}), 537(\mathrm{~m}), 515(\mathrm{w})$.

\section{1-(Toluene-4-sulfonyl)-2-vinyl-1H-indole (95)}

Following a reported procedure, ${ }^{14} \mathrm{BuLi}(1.6 \mathrm{M}$ in hexane, $2.7 \mathrm{~mL}, 4.3 \mathrm{mmol}, 1.1$ equiv) was added to a suspension of methyltriphenylphosphonium bromide $\left(1.7 \mathrm{~g}, 4.8 \mathrm{mmol}, 1.2\right.$ equiv) in THF $(40 \mathrm{~mL})$ at $0{ }^{\circ} \mathrm{C}$ under argon. After stirring for $2 \mathrm{~h}$, the reaction mixture was cooled to $-78{ }^{\circ} \mathrm{C}$ and a solution of $94(1.2 \mathrm{~g}, 3.9 \mathrm{mmol}, 1.0$ equiv) in THF (5 mL) was added dropwise. The reaction was let to warm up to $23{ }^{\circ} \mathrm{C}$ over $12 \mathrm{~h}$, quenched with water $(60 \mathrm{~mL})$ and extracted with $\mathrm{Et}_{2} \mathrm{O}(3 \times 60 \mathrm{~mL})$. The combined organic layer were washed with water $(30 \mathrm{~mL})$ and brine $(30 \mathrm{~mL})$, dried over $\mathrm{Na}_{2} \mathrm{SO}_{4}$ and the solvent was removed under reduced pressure. The crude product was purified by column chromatography (AcOEt/hexane 1:20) to give $95(0.98 \mathrm{~g}, 3.3 \mathrm{mmol}, 85 \%)$ as a colorless solid. Mp 93-94 ${ }^{\circ} \mathrm{C} ;{ }^{1} \mathrm{H}$ NMR $\left(\mathrm{CDCl}_{3}, 300 \mathrm{MHz}\right) \delta 8.01(\mathrm{dm}, J=8.1 \mathrm{~Hz}, 1 \mathrm{H}$, indole $\mathrm{H}), 7.82-7.73(\mathrm{~m}, 1 \mathrm{H}$, indole $\mathrm{H})$, $7.78(\mathrm{~d}, J=8.7 \mathrm{~Hz}, 2 \mathrm{H}$, phenyl H), $7.62(\mathrm{~s}, 1 \mathrm{H}$, indole $\mathrm{H}), 7.37-7.24(\mathrm{~m}, 2 \mathrm{H}$, indole $\mathrm{H}), 7.20(\mathrm{~d}, J=7.8 \mathrm{~Hz}, 2 \mathrm{H}, \mathrm{phenyl} \mathrm{H}), 6.77$ $(\mathrm{ddm}, J=17.7,11.2 \mathrm{~Hz}, 1 \mathrm{H}$, vinyl $\mathrm{H}), 5.80(\mathrm{dm}, J=18.0 \mathrm{~Hz}, 1 \mathrm{H}$, vinyl $\mathrm{H}), 5.35(\mathrm{dm}, J=11.2 \mathrm{~Hz}, 1 \mathrm{H}$, vinyl H), $2.31(\mathrm{~s}, 3 \mathrm{H}$, $\left.\mathrm{CH}_{3}\right) ;{ }^{13} \mathrm{C} \mathrm{NMR}\left(\mathrm{CDCl}_{3}, 75 \mathrm{MHz}\right) \delta 144.9,135.3,134.9,129.7,129.6,128.8,127.7,127.4,126.7,124.8,123.9,123.4,120.8$, 115.2, 113.6, 21.6; IR v 3126 (w), 3066 (w), 2961 (w), 2925 (w), 1917 (w), 1637 (w), 1597 (w), 1542 (w), $1494(w), 1447$ (m), $1416(\mathrm{w}), 1400(\mathrm{w}), 1373(\mathrm{~s}), 1306(\mathrm{w}), 1293(\mathrm{w}), 1268(\mathrm{~m}), 1217(\mathrm{~m}), 1188(\mathrm{~s}), 1176(\mathrm{~s}), 1146(\mathrm{w}), 1124(\mathrm{~s}), 1089(\mathrm{~m}), 1042$ (w), $1020(\mathrm{w}), 989(\mathrm{w}), 962(\mathrm{~m}), 939(\mathrm{w}), 907(\mathrm{w}), 812(\mathrm{~m}), 766(\mathrm{~m}), 747(\mathrm{~m}), 703(\mathrm{w}), 668(\mathrm{~s}), 625(\mathrm{w}), 594(\mathrm{~m}), 575(\mathrm{~s}), 536$ (m), $510(\mathrm{w}), 487(\mathrm{w})$.

2-Vinyl-indole-1-carboxylic acid tert-butyl ester $(97)^{19}$ 


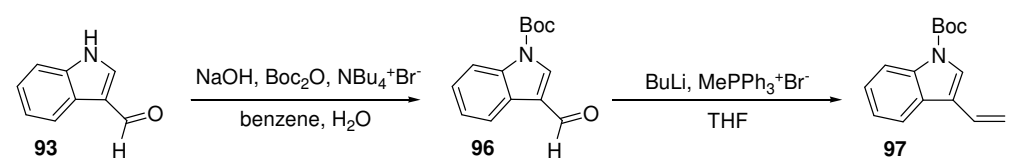

2-Formyl-indole-1-carboxylic acid tert-butyl ester $(96)^{20}$

Following a reported procedure, ${ }^{18}$ aqueous $30 \% \mathrm{NaOH}(25 \mathrm{~mL})$, Boc anhydride $(1.6 \mathrm{~g}, 7.2 \mathrm{mmol}$, 1.1 equiv) and tetrabutylammonium bromide $(0.22 \mathrm{~g}, 0.69 \mathrm{mmol}, 0.10$ equiv) were added to a suspension of indole aldehyde 93 ( $1.0 \mathrm{~g}, 6.9 \mathrm{mmol}$, 1.0 equiv) in benzene $(25 \mathrm{~mL})$. After stirring vigorously for $30 \mathrm{~min}$, the layers were separated and the water layer was extracted with benzene $(10 \mathrm{~mL})$. The combined organic layers were dried over $\mathrm{Na}_{2} \mathrm{SO}_{4}$ and the solvent was removed under reduced pressure. The crude product was purified by recrystallization from AcOEt/hexane to afford aldehyde 96 (1.05 g, $4.28 \mathrm{mmol}, 62 \%)$ as a colorless solid. Mp (AcOEt/hexane) $124-126{ }^{\circ} \mathrm{C}$ (Lit.: $\left.{ }^{18} 123-125{ }^{\circ} \mathrm{C}\right) ;{ }^{1} \mathrm{H} \mathrm{NMR}\left(\mathrm{CDCl}_{3}, 300 \mathrm{MHz}\right) \delta 10.10(\mathrm{~s}, 1 \mathrm{H}$, aldehyde $\mathrm{H}), 8.29(\mathrm{~m}, 1 \mathrm{H}$, indole $\mathrm{H}), 8.24(\mathrm{~s}, 1 \mathrm{H}$, indole $\mathrm{H}), 8.15(\mathrm{~d}, J=7.8 \mathrm{~Hz}, 1 \mathrm{H}$, indole $\mathrm{H}), 7.45-7.34(\mathrm{~m}, 2 \mathrm{H}$, indole $\mathrm{H}), 1.71(\mathrm{~s}, 9 \mathrm{H}$, $\left.\mathrm{CH}_{3}\right) ;{ }^{13} \mathrm{C} \mathrm{NMR}\left(\mathrm{CDCl}_{3}, 75 \mathrm{MHz}\right) \delta 185.4,148.5,136.3,135.7,125.8,124.3,121.8,121.3,114.9,85.5,28.0 ; \mathrm{IR} v 3144(\mathrm{w}), 3077$ (w), 3054 (w), 3002 (w), 2979 (m), 2939 (w), $2816(\mathrm{~m}), 2763$ (w), 2740 (w), 1967 (w), 1928 (w), 1829 (w), 1806 (w), 1746 (s), 1681 (s), 1609 (w), 1558 (m), 1481 (m), 1452 (m), 1434 (w), 1398 (s), 1369 (s), 1332 (m), 1310 (m), 1276 (m), 1259 (s), 1243 (s), 1155 (s), 1134 (s), 1101 (s), 1046 (w), 1017 (w), 946 (w), 909 (m), 840 (s), 786 (m), 761 (s), 750 (s), 668 (w), 649 (w), 596 (w), $518(\mathrm{w})$.

\section{2-Vinyl-indole-1-carboxylic acid tert-butyl ester (97)}

Following a reported procedure, ${ }^{14} \mathrm{BuLi}(1.6 \mathrm{M}$ in hexane, $2.6 \mathrm{~mL}, 4.2 \mathrm{mmol}, 1.1$ equiv) was added to a suspension of methyltriphenylphosphonium bromide ( $1.6 \mathrm{~g}, 4.5 \mathrm{mmol}, 1.2$ equiv) in THF ( $40 \mathrm{~mL})$ at $0{ }^{\circ} \mathrm{C}$ under argon. After stirring for $2 \mathrm{~h}$, the reaction mixture was cooled to $-78{ }^{\circ} \mathrm{C}$ and a solution of $96(0.90 \mathrm{~g}, 3.7 \mathrm{mmol}, 1.0$ equiv) in THF $(5 \mathrm{~mL})$ was added dropwise. The reaction was let to warm up to $23{ }^{\circ} \mathrm{C}$ over $12 \mathrm{~h}$, quenched with water $(60 \mathrm{~mL})$ and extracted with $\mathrm{Et}_{2} \mathrm{O}(3 \times 60 \mathrm{~mL})$. The combined organic layer were washed with water $(30 \mathrm{~mL})$ and brine $(30 \mathrm{~mL})$, dried over $\mathrm{Na}_{2} \mathrm{SO}_{4}$ and the solvent was removed under reduced pressure. The crude product was purified by column chromatography (AcOEt/hexane 1:30) to give $97(0.72 \mathrm{~g}, 3.0 \mathrm{mmol}, 80 \%)$ as a yellow oil. ${ }^{1} \mathrm{H} \mathrm{NMR}\left(\mathrm{CDCl}_{3}, 300 \mathrm{MHz}\right) \delta 8.21(\mathrm{~d}, J=7.9 \mathrm{~Hz}, 1 \mathrm{H}$, indole $\mathrm{H}), 7.81(\mathrm{~d}, J=7.6 \mathrm{~Hz}, 1 \mathrm{H}$, indole $\mathrm{H}), 7.65(\mathrm{~s}, 1 \mathrm{H}$, indole $\mathrm{H}), 7.39-7.25(\mathrm{~m}, 2 \mathrm{H}$, indole $\mathrm{H}), 6.83(\mathrm{dd}, J=17.8,11.3 \mathrm{~Hz}, 1 \mathrm{H}$, vinyl $\mathrm{H}), 5.84(\mathrm{~d}, J=17.8 \mathrm{~Hz}, 1 \mathrm{H}$, vinyl H), $5.35(\mathrm{~d}, J=$ $11.3 \mathrm{~Hz}, 1 \mathrm{H}$, vinyl H), 1.70 (s, 9H, $\left.\mathrm{CH}_{3}\right) ;{ }^{13} \mathrm{C} \mathrm{NMR}\left(\mathrm{CDCl}_{3}, 75 \mathrm{MHz}\right) \delta 149.4,135.8,128.5,128.0,124.4,123.8,122.8,119.8$, 119.1, 115.2, 114.2, 83.7, 28.2; IR v 3139 (w), 3083 (w), 3053 (w), 2979 (m), 2933 (w), 1732 (s), 1637 (m), 1607 (w), 1558 (w), $1550(\mathrm{w}), 1477(\mathrm{~m}), 1453(\mathrm{~s}), 1418(\mathrm{~m}), 1371(\mathrm{~s}), 1326(\mathrm{~m}), 1308(\mathrm{~s}), 1255(\mathrm{~s}), 1235(\mathrm{~s}), 1225(\mathrm{~s}), 1158(\mathrm{~s}), 1100(\mathrm{~s}), 1079(\mathrm{~m})$, $1049(\mathrm{~m}), 1034(\mathrm{w}), 1024(\mathrm{~m}), 988(\mathrm{~m}), 935(\mathrm{w}), 898(\mathrm{w}), 857(\mathrm{~m}), 836(\mathrm{w}), 814(\mathrm{w}), 765(\mathrm{~m}), 746(\mathrm{~s}), 706(\mathrm{w}), 686(\mathrm{w}), 639(\mathrm{w})$, $590(\mathrm{w}), 569(\mathrm{w}), 534(\mathrm{w}), 512(\mathrm{w}), 471(\mathrm{w}), 441(\mathrm{w})$.

2-Isopropenyl-pyridine $(99)^{21}$

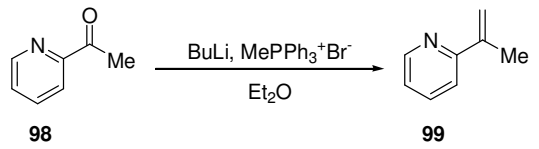

Following a reported procedure, ${ }^{22} \mathrm{BuLi}(1.6 \mathrm{M}$ in hexane, $10 \mathrm{~mL}, 16 \mathrm{mmol}, 1.0$ equiv) was added to a suspension of methyltriphenylphosphonium bromide $\left(5.7 \mathrm{~g}, 16 \mathrm{mmol}, 1.0\right.$ equiv) in $\mathrm{Et}_{2} \mathrm{O}(20 \mathrm{~mL})$ at $0{ }^{\circ} \mathrm{C}$ under argon. The reaction mixture was warmed to $23{ }^{\circ} \mathrm{C}$ and stirred for $40 \mathrm{~min}$. A solution of $98\left(2.0 \mathrm{~g}, 16 \mathrm{mmol}, 1.0\right.$ equiv) in $\mathrm{Et}_{2} \mathrm{O}(15 \mathrm{~mL})$ was added dropwise, followed by $\mathrm{Et}_{2} \mathrm{O}(15 \mathrm{~mL})$. After stirring at reflux for $2 \mathrm{~h}$, the mixture was quenched with water $(100 \mathrm{~mL})$ and extracted with $\mathrm{Et}_{2} \mathrm{O}$ $(2 \times 150 \mathrm{~mL})$. The combined organic layers were dried over $\mathrm{Na}_{2} \mathrm{SO}_{4}$ and the solvent was removed under reduced pressure. The crude product was purified by column chromatography (AcOEt/hexane 1:1) to give pyridine $99(0.35 \mathrm{~g}, 2.9 \mathrm{mmol}, 18 \%)$ as an oil. ${ }^{1} \mathrm{H} \mathrm{NMR}\left(\mathrm{CDCl}_{3}, 300 \mathrm{MHz}\right) \delta 8.59(\mathrm{dq}, J=4.8,0.9 \mathrm{~Hz}, 1 \mathrm{H}$, pyridine $\mathrm{H}), 7.65$ (ddd, $J=8.0,7.5,1.9 \mathrm{~Hz}, 1 \mathrm{H}$, pyridine H), 7.49 (dt, $J=8.0,1.1 \mathrm{~Hz}, 1 \mathrm{H}$, pyridine $\mathrm{H}$ ), 7.17 (ddd, $J=7.4,4.8,1.2 \mathrm{~Hz}, 1 \mathrm{H}$, pyridine $\mathrm{H}$ ), 5.85 (sextet, $J=0.8 \mathrm{~Hz}, 1 \mathrm{H}$, vinyl H), 5.30 , (qi, $J=1.6 \mathrm{~Hz}, 1 \mathrm{H}$, vinyl H), $2.22\left(\mathrm{dd}, J=1.5,0.8 \mathrm{~Hz}, 3 \mathrm{H}, \mathrm{CH}_{3}\right) ;{ }^{13} \mathrm{C} \mathrm{NMR}\left(\mathrm{CDCl}_{3}, 75 \mathrm{MHz}\right) \delta 158.0,148.7,143.0,136.1,121.9$, $120.0,115.5,20.5$.

\section{2-Propenyl-pyridine $(101)^{23}$}

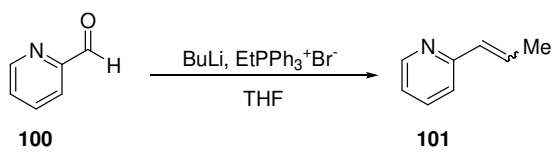

Following a reported procedure, ${ }^{23} \mathrm{BuLi}(1.6 \mathrm{M}$ in hexane, $12 \mathrm{~mL}, 19 \mathrm{mmol}, 1.0$ equiv) was added to a suspension of ethyltriphenylphosphonium bromide ( $7.3 \mathrm{~g}, 20 \mathrm{mmol}, 1.1$ equiv) in THF $(35 \mathrm{~mL})$ at $23{ }^{\circ} \mathrm{C}$ under argon. After $30 \mathrm{~min}$, a solution of $100\left(2.0 \mathrm{~g}, 19 \mathrm{mmol}, 1.0\right.$ equiv) in THF $(10 \mathrm{~mL})$ was added dropwise. After stirring at $23{ }^{\circ} \mathrm{C}$ for $66 \mathrm{~h}$, the mixture was quenched with water $(100 \mathrm{~mL})$, THF was removed under reduced pressure and the reaction mixture was extracted with $\mathrm{CH}_{2} \mathrm{Cl}_{2}$ $(3 \times 75 \mathrm{~mL})$. The combined organic layers were dried over $\mathrm{Na}_{2} \mathrm{SO}_{4}$ and the solvent was removed under reduced pressure. The crude product was purified by column chromatography (AcOEt/hexane 1:1) to give pyridine $101(1.13 \mathrm{~g}, 9.45 \mathrm{mmol}, 50 \%, 2: 1 \mathrm{E} / \mathrm{Z}$ mixture) as an oil. ${ }^{1} \mathrm{H} \mathrm{NMR}\left(\mathrm{CDCl}_{3}, 300 \mathrm{MHz}\right) \delta 8.60(\mathrm{~d}, J=4.5 \mathrm{~Hz}, 1 \mathrm{H}(33 \%)$, pyridine $\mathrm{H}(Z)), 8.51(\mathrm{~d}, J=4.5 \mathrm{~Hz}, 1 \mathrm{H}(66 \%)$, pyridine $\mathrm{H}(E))$, 7.66-7.56 $(\mathrm{m}, 1 \mathrm{H}$, pyridine $\mathrm{H}(E$ and $Z))$, 7.32-7.20 $(\mathrm{m}, 1 \mathrm{H}$, pyridine $\mathrm{H}(E$ and $Z)), 7.11-7.06(\mathrm{~m}, 1 \mathrm{H}$, pyridine $\mathrm{H}$ 
$(E$ and $Z)), 6.80-6.68(\mathrm{~m}, 1 \mathrm{H}(66 \%)$, vinyl $\mathrm{H}(E)), 6.52-6.47(\mathrm{~m}, 1 \mathrm{H}$, vinyl $\mathrm{H}(E$ and $Z)), 6.06-5.95(\mathrm{~m}, 1 \mathrm{H}(33 \%)$, vinyl $\mathrm{H}(Z))$, $2.09\left(\mathrm{dd}, J=7.2,1.3 \mathrm{~Hz}, 3 \mathrm{H}(33 \%), \mathrm{CH}_{3}(Z)\right), 1.93\left(\mathrm{~d}, J=6.6 \mathrm{~Hz}, 3 \mathrm{H}(66 \%), \mathrm{CH}_{3}(E)\right) ;{ }^{13} \mathrm{C} \mathrm{NMR}\left(\mathrm{CDCl}_{3}, 75 \mathrm{MHz} \delta 156.5\right.$, $155.8,149.1,148.9,136.0,135.6,131.1,130.9,130.4,129.3,123.6,121.2,120.8,120.6,18.3,15.0$.

2-Isopropenyl-thiophene $(\mathbf{1 0 3})^{24}$

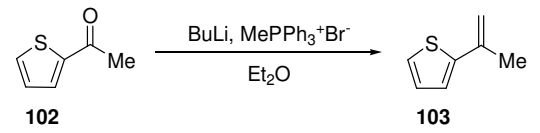

Following a reported procedure, ${ }^{22} \mathrm{BuLi}(1.6 \mathrm{M}$ in hexane, $10 \mathrm{~mL}, 16 \mathrm{mmol}, 1.0$ equiv) was added to a suspension of methyltriphenylphosphonium bromide $\left(5.7 \mathrm{~g}, 16 \mathrm{mmol}, 1.0\right.$ equiv) in $\mathrm{Et}_{2} \mathrm{O}(15 \mathrm{~mL})$ at $0{ }^{\circ} \mathrm{C}$ under argon. The reaction mixture was warmed to $23{ }^{\circ} \mathrm{C}$ and stirred for $40 \mathrm{~min}$. A solution of $102\left(2.1 \mathrm{~g}, 16 \mathrm{mmol}, 1.0\right.$ equiv) in $\mathrm{Et}_{2} \mathrm{O}(20 \mathrm{~mL})$ was added dropwise. After stirring at reflux for $2 \mathrm{~h}$, the mixture was quenched with water $(100 \mathrm{~mL})$ and extracted with $\mathrm{Et}_{2} \mathrm{O}(2 \times 150 \mathrm{~mL})$. The combined organic layers were dried over $\mathrm{Na}_{2} \mathrm{SO}_{4}$ and the solvent was removed under reduced pressure. The crude product was purified by column chromatography $\left(\mathrm{CH}_{2} \mathrm{Cl}_{2} /\right.$ hexane $\left.10: 1\right)$ to give thiophene $\mathbf{1 0 3}(0.52 \mathrm{~g}, 4.2 \mathrm{mmol}, 25 \%)$ as an oil. ${ }^{1} \mathrm{H} \mathrm{NMR}$ $\left(\mathrm{CDCl}_{3}, 300 \mathrm{MHz}\right) \delta 7.18(\mathrm{~d}, J=5.1 \mathrm{~Hz}, 1 \mathrm{H}$, thiophene $\mathrm{H}), 7.05-6.97(\mathrm{~m}, 2 \mathrm{H}$, thiophene $\mathrm{H}), 5.40(\mathrm{~s}, 1 \mathrm{H}$, vinyl $\mathrm{H}), 4.97(\mathrm{~s}, 1 \mathrm{H}$, vinyl $\mathrm{H}), 2.17\left(\mathrm{~s}, 3 \mathrm{H}, \mathrm{CH}_{3}\right) ;{ }^{13} \mathrm{C} \mathrm{NMR}\left(\mathrm{CDCl}_{3}, 75 \mathrm{MHz}\right) \delta 146.0,137.4,127.5,124.4,123.8,111.4,22.0$.

2-Isopropenyl-benzothiazole $(21)^{25}$
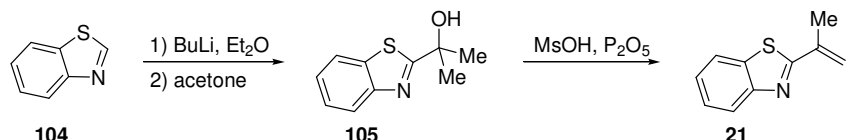

\section{2-Benzothiazol-2-yl-propan-2-ol (105) ${ }^{25}$}

Following a reported procedure, ${ }^{25}$ benzothiazole (104) (freshly distilled, $3.0 \mathrm{~mL}, 27 \mathrm{mmol}, 1.0$ equiv) was added dropwise over $20 \mathrm{~min}$ to a mixture of $\mathrm{BuLi}\left(1.6 \mathrm{M}\right.$ in hexane, $18 \mathrm{~mL}, 29 \mathrm{mmol}, 1.1$ equiv) and $\mathrm{Et}_{2} \mathrm{O}(60 \mathrm{~mL})$ at $-78{ }^{\circ} \mathrm{C}$ under argon. After 25 min, a solution of acetone $\left(2.2 \mathrm{~mL}, 30 \mathrm{mmol}, 1.1\right.$ equiv) in $\mathrm{Et}_{2} \mathrm{O}(10 \mathrm{~mL})$ was added dropwise and the resulting suspension was stirred below $-50{ }^{\circ} \mathrm{C}$ for $3 \mathrm{~h}$. The reaction mixture was quenched with sat. $\mathrm{NH}_{4} \mathrm{Cl}(5 \mathrm{~mL})$ and heated to $23{ }^{\circ} \mathrm{C}$. $\mathrm{Sat}$. $\mathrm{NH}_{4} \mathrm{Cl}(50 \mathrm{~mL})$ and water $(10 \mathrm{~mL})$ were added the layers were separated and the water layer was extracted with $\mathrm{Et}_{2} \mathrm{O}(50 \mathrm{~mL})$. The combined organic layers were dried over $\mathrm{Na}_{2} \mathrm{SO}_{4}$ and the solvent was removed under reduced pressure. The crude product was purified by recrystallization from hexane to afford alcohol $105(4.8 \mathrm{~g}, 25 \mathrm{mmol}, 92 \%)$ as slightly yellow crystals. ${ }^{1} \mathrm{H}$ NMR $(\mathrm{CDCl}, 300$ MHz) $\delta 7.98(\mathrm{dm}, J=8.1 \mathrm{~Hz}, 1 \mathrm{H}, \mathrm{Ar} \mathrm{H}), 7.88(\mathrm{dm}, J=8.1 \mathrm{~Hz}, 1 \mathrm{H}, \mathrm{Ar} \mathrm{H}), 7.49-7.43(\mathrm{~m}, 1 \mathrm{H}, \mathrm{Ar} \mathrm{H}), 7.39-7.33(\mathrm{~m}, 1 \mathrm{H}, \mathrm{Ar} \mathrm{H})$, 3.29 (br s, $1 \mathrm{H}, \mathrm{OH}), 1.75\left(\mathrm{~s}, 6 \mathrm{H}, \mathrm{CH}_{3}\right) ;{ }^{13} \mathrm{C} \mathrm{NMR}\left(\mathrm{CDCl}_{3}, 75 \mathrm{MHz}\right) \delta 180.0,152.8,135.1,125.8,124.7,122.7,121.6,73.5,30.8$; IR v $3370(\mathrm{~m}), 3064(\mathrm{w}), 2978(\mathrm{~m}), 2932(\mathrm{w}), 1910(\mathrm{w}), 1788(\mathrm{w}), 1594(\mathrm{w}), 1560(\mathrm{w}), 1515(\mathrm{~m}), 1456(\mathrm{~m}), 1439(\mathrm{~s}), 1363(\mathrm{~m})$, $1315(\mathrm{~m}), 1277$ (m), 1243 (s), 1181 (s), $1142(\mathrm{~m}), 1091(\mathrm{~m}), 1048(\mathrm{~s}), 1015(\mathrm{w}), 961$ (m), $871(\mathrm{w}), 825$ (w), $759(\mathrm{~s}), 730(\mathrm{~s}), 698$ (w), $656(\mathrm{w}), 621(\mathrm{w}), 540(\mathrm{w}), 460(\mathrm{w})$.

\section{2-Isopropenyl-benzothiazole (21)}

Phosphorous pentoxide ( $2.0 \mathrm{~g}$ ) was added to methanesulfonic acid $(20 \mathrm{~g})$ at $23{ }^{\circ} \mathrm{C}$ under argon and the resulting suspension was stirred for $12 \mathrm{~h}$ and became a clear solution. Alcohol 105 (1.54 g, $7.97 \mathrm{mmol}, 1.00$ equiv) was added and the reaction mixture was heated to $60{ }^{\circ} \mathrm{C}$ for $10 \mathrm{~h}$. The reaction mixture was cooled to $23{ }^{\circ} \mathrm{C}$ and poured onto sat. $\mathrm{NaHCO}_{3}(50 \mathrm{~mL})$ at $0{ }^{\circ} \mathrm{C}$. Solid $\mathrm{NaHCO}$ was added until gas evolution stopped. The reaction mixture was extracted with $\mathrm{CH}_{2} \mathrm{Cl}_{2}(3 \times 50 \mathrm{~mL})$. The combined organic layers were dried over $\mathrm{Na}_{2} \mathrm{SO}_{4}$ and the solvent was removed under reduced pressure. The crude product was purified by column chromatography (AcOEt/hexane 1:20) to give benzothiazole $21(1.17 \mathrm{~g}, 6.64 \mathrm{mmol}, 83 \%)$ as a low melting solid. Mp 25-30 ${ }^{\circ} \mathrm{C}$; ${ }^{1} \mathrm{H}$ NMR $\left(\mathrm{CDCl}_{3}, 300 \mathrm{MHz}\right) \delta 8.02(\mathrm{dm}, J=8.1 \mathrm{~Hz}, 1 \mathrm{H}, \mathrm{Ar} \mathrm{H}), 7.84(\mathrm{dm}, J=8.1 \mathrm{~Hz}, 1 \mathrm{H}, \mathrm{Ar} \mathrm{H}), 7.49-7.33(\mathrm{~m}, 2 \mathrm{H}, \mathrm{Ar} \mathrm{H}), 5.96$ $(\mathrm{s}, 1 \mathrm{H}$, alkene $\mathrm{H}), 5.52(\mathrm{~m}, 1 \mathrm{H}$, alkene $\mathrm{H}), 2.34\left(\mathrm{~m}, 3 \mathrm{H}, \mathrm{CH}_{3}\right) ;{ }^{13} \mathrm{C} \mathrm{NMR}\left(\mathrm{CDCl}_{3}, 75 \mathrm{MHz}\right) \delta 169.2,153.6,138.9,134.6,125.9$, 125.2, 123.1, 121.3, 119.7, 20.4; IR v 3062 (w), 2979 (w), 2959 (w), 2922 (w), 1816 (w), 1629 (w), 1592 (w), 1557 (w), 1494 (s), $1456(\mathrm{~m}), 1435(\mathrm{~m}), 1375(\mathrm{w}), 1317(\mathrm{~m}), 1298(\mathrm{w}), 1274(\mathrm{w}), 1244(\mathrm{w}), 1160(\mathrm{w}), 1124(\mathrm{w}), 1088(\mathrm{~m}), 1050(\mathrm{~m}), 1016(\mathrm{w}), 995$ (w), $903(\mathrm{~m}), 860$ (w), $758(\mathrm{~s}), 728$ (s), $697(\mathrm{w}), 626(\mathrm{w}), 603(\mathrm{w}), 550(\mathrm{w}), 502(\mathrm{w})$.

1,2-Dimethyl-cyclohexene $(\mathbf{1 0 8})^{26}$
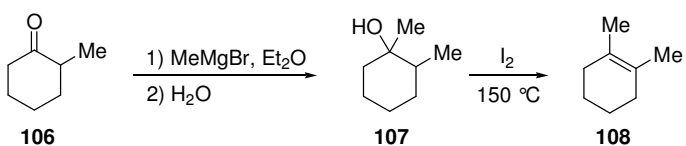

\section{1,2-Dimethyl-cyclohexanol (107)}

2-Methyl-cyclohexanone (106) $\left(5.0 \mathrm{~mL}, 41 \mathrm{mmol}\right.$, freshly distilled, 1.0 equiv) was diluted in $\mathrm{Et}_{2} \mathrm{O}\left(9 \mathrm{~mL}\right.$, dry) at $0{ }^{\circ} \mathrm{C}$ under argon and a solution of methylmagnesium bromide $\left(3 \mathrm{M}^{\text {in }} \mathrm{Et}_{2} \mathrm{O}, 16 \mathrm{~mL}, 48 \mathrm{mmol}, 1.2\right.$ equiv) was added dropwise over $45 \mathrm{~min}$. The reaction mixture was then allowed to warm to $23{ }^{\circ} \mathrm{C}$ over night, quenched with a crushed ice/water mixture and the $\mathrm{pH}$ was adjusted to 1 with concentrated $\mathrm{HCl}$. The layers were separated and the organic layer was washed with sat. $\mathrm{NaHSO}_{3}$ solution $(2 \times 10 \mathrm{~mL})$, water $(4 \times 10 \mathrm{~mL})$, brine $(10 \mathrm{~mL})$, dried over $\mathrm{Na}_{2} \mathrm{SO}_{4}$, filtered and the solvents were removed under reduced pressure. 
The crude product was purified by distillation ( $\left.p=100 \mathrm{mbar}, b p=80{ }^{\circ} \mathrm{C}\right)$ to afford $\mathbf{1 0 7}(4.4 \mathrm{~g}, 34 \mathrm{mmol}, 83 \%, 1: 1 \mathrm{mixture}$ of diastereomers) as a slightly yellow liquid. ${ }^{1} \mathrm{H}$ NMR $\left(\mathrm{CDCl}_{3}, 300 \mathrm{MHz}\right)^{26 \mathrm{~b}} \delta 1.70-1.10(\mathrm{~m}, 10 \mathrm{H}), 1.19\left(\mathrm{~s}, 2.25 \mathrm{H}, \mathrm{COHCH}_{3}\right.$, major diastereoisomer), $1.10\left(0.7 \mathrm{H}, \mathrm{s}, \mathrm{COHCH}_{3}\right.$, minor diastereoisomer), $0.93\left(\mathrm{~d}, 0.75 \mathrm{H}, J=6.9 \mathrm{~Hz}, \mathrm{CHCH}_{3}\right.$, minor diastereoisomer), 0.91 (d, $J=6.2 \mathrm{~Hz}, \mathrm{CHCH}_{3}$, major diastereoisomer).

\section{1,2-Dimethyl-cyclohexene (108)}

1,2-Dimethyl-cyclohexanol (107) (4.1 g, $32 \mathrm{mmol}, 1.0$ equiv) and iodine (50 mg, $0.20 \mathrm{mmol}, 0.0060$ equiv) were mixed at 23 ${ }^{\circ} \mathrm{C}$ under argon and the solution was heated to $150{ }^{\circ} \mathrm{C}$ and directly distilled ( $p=100 \mathrm{mbar}, b p=60{ }^{\circ} \mathrm{C}$ ). The distilled liquid was dried $2 \mathrm{~h}$ over $\mathrm{CaCl}_{2}$ and filtered to give 1,2-dimethyl-cyclohexene (108) together with 1,6-dimethyl-cyclohexene (5:1 ratio, $3.1 \mathrm{~g}$, $28 \mathrm{mmol}, 89 \%)$ as a colorless liquid. ${ }^{1} \mathrm{H}$ NMR $\left(\mathrm{CDCl}_{3}, 300 \mathrm{MHz}\right)^{26 \mathrm{c}} \delta 2.06-0.90\left(\mathrm{~m}, 8 \mathrm{H}, \mathrm{CH}_{2}\right), 1.60\left(\mathrm{~s}, 6 \mathrm{H}, \mathrm{CH}_{3}\right)$.

\section{$1,2,3,4,5,6,7,8-O c t a h y d r o-n a p h t h a l e n e ~(110)^{27}$}

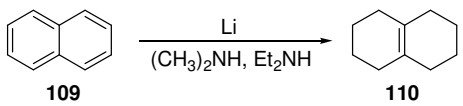

Naphthalene (109) $\left(2.6 \mathrm{~g}, 20 \mathrm{mmol}, 1.0\right.$ equiv) was dissolved in dimethylamine $(25 \mathrm{~mL})$ and ethylamine $(25 \mathrm{~mL})$ at $0{ }^{\circ} \mathrm{C}$ under argon. Lithium ( $1.2 \mathrm{~g}, 0.17 \mathrm{~mol}, 8.3$ equiv, wires, freshly cut in $0.5 \mathrm{~cm}$ pieces and washed with hexane) was added over $45 \mathrm{~min}$, whereas the reaction mixture turned red, then blue-red. The reaction mixture was stirred at reflux $\left(15^{\circ} \mathrm{C}\right.$, dry ice cooling $)$ for $13 \mathrm{~h}$, the dry ice condenser was removed and the solvents were evaporated at $23{ }^{\circ} \mathrm{C}$ over night. The greyish residues were quenched with water $\left(50 \mathrm{~mL}\right.$, caution, violent reaction!) and the suspension was filtered, the residues were washed with $\mathrm{Et}_{2} \mathrm{O}(3 \times 10 \mathrm{~mL})$, the layers were separated, the water layer was extracted with $\mathrm{Et}_{2} \mathrm{O}(5 \times 20 \mathrm{~mL})$, the combined organic layers were dried over $\mathrm{Na}_{2} \mathrm{SO}_{4}$ and the solvents were removed under reduced pressure $\left(p=100 \mathrm{mbar}, T=40{ }^{\circ} \mathrm{C}\right)$. The crude product was distilled $(p=$ $\left.20 \mathrm{mbar}, b p=80^{\circ} \mathrm{C}\right)$ to give octahydronaphtalene $\mathbf{1 1 0}(1.9 \mathrm{~g}, 14 \mathrm{mmol}, 70 \%)$ as a 5.5:1 mixture of isomers.

$\mathrm{NaBH}_{4}\left(0.24 \mathrm{~g}, 6.2 \mathrm{mmol}, 0.60\right.$ equiv) was suspended in THF $(12 \mathrm{~mL})$ at $23{ }^{\circ} \mathrm{C}$ under argon and 2-methyl-2-butene $(1.7 \mathrm{~mL}, 16$ mmol, 1.5 equiv) and a solution of $\mathrm{BF}_{3} \bullet \mathrm{Et}_{2} \mathrm{O}(1.0 \mathrm{~mL}, 8.3 \mathrm{mmol}, 0.75$ equiv, freshly distilled) in THF (3.0 mL) were added dropwise. The crude octahydronaphtalene $(1.8 \mathrm{~g}, 11 \mathrm{mmol} \mathbf{1 1 0}, 1$ equiv) was added dropwise to the resulting suspension, the reaction mixture was stirred at $23{ }^{\circ} \mathrm{C}$ for $2 \mathrm{~h}$ and quenched with water $(5 \mathrm{~mL})$ and $3 \mathrm{M} \mathrm{NaOH}(3.5 \mathrm{~mL}) .30 \% \mathrm{H}_{2} \mathrm{O}_{2}(3.5 \mathrm{~mL})$ was then added dropwise, the reaction mixture was heated to $45^{\circ} \mathrm{C}$ and stirred at that temperature for $5 \mathrm{~h}$. After cooling to $23{ }^{\circ} \mathrm{C}$, the layers were separated, the water layer extracted with $\mathrm{Et}_{2} \mathrm{O}(2 \times 10 \mathrm{~mL})$, the combined organic layers washed with water $(4 \times 10 \mathrm{~mL})$, dried over $\mathrm{Na}_{2} \mathrm{SO}_{4}$ and the solvents removed under reduced pressure $\left(p=100 \mathrm{mbar}, T=40{ }^{\circ} \mathrm{C}\right)$. Distillation $(p=20 \mathrm{mbar}, b p=80$ ${ }^{\circ} \mathrm{C}$ furnished $110(1.2 \mathrm{~g}, 9.0 \mathrm{mmol}, 82 \%)$ containing less than $7 \%$ of 2,3,4,5,6,7,9-Octahydro-naphthalene. ${ }^{1} \mathrm{H} \mathrm{NMR}\left(\mathrm{CDCl}{ }_{3}, 300\right.$ $\mathrm{MHz})^{27 \mathrm{~b}} \delta 1.84\left(\mathrm{~m}, 8 \mathrm{H}, \mathrm{CH}_{2}\right), 1.59\left(\mathrm{~m}, 8 \mathrm{H}, \mathrm{CH}_{2}\right)$.

3-Methyl-2-phenyl-but-2-ene (112) ${ }^{28}$

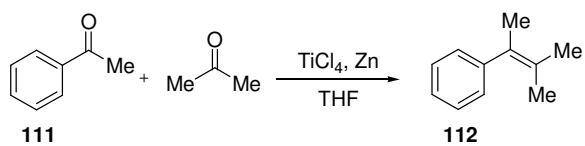

$\mathrm{TiCl}_{4}\left(6.0 \mathrm{~mL}, 55 \mathrm{mmol}, 5.5\right.$ equiv, freshly distilled) was added dropwise to THF $(150 \mathrm{~mL}$, dry $)$ at $0{ }^{\circ} \mathrm{C}$ under argon over 40 min and a yellow suspension was formed in a violent reaction. After stirring for $20 \mathrm{~min}, \mathrm{Zn}(7.3 \mathrm{~g}, 0.11 \mathrm{~mol}, 11 \mathrm{equiv}$, activated) was added in small portions at $0{ }^{\circ} \mathrm{C}$ over $1 \mathrm{~h}$, whereas the reaction mixture turned black-red. A mixture of acetophenone (111) $(1.2 \mathrm{~mL}, 9.7 \mathrm{mmol}, 1.0$ equiv) and acetone $(3.0 \mathrm{~mL}, 41 \mathrm{mmol}, 4.2$ equiv, freshly distilled) in THF (20 mL) was added dropwise over $30 \mathrm{~min}$ and the reaction mixture was heated to reflux for $22 \mathrm{~h}$. After cooling to $23{ }^{\circ} \mathrm{C}$, the reaction was quenched with $10 \%$ $\mathrm{K}_{2} \mathrm{CO}_{3}$ solution $(150 \mathrm{~mL})$, the layers were separated, the water layer extracted with $\mathrm{Et}_{2} \mathrm{O}(4 \times 50 \mathrm{~mL})$, the combined organic layers washed with water $(50 \mathrm{~mL})$, brine $(50 \mathrm{~mL})$, dried over $\mathrm{Na}_{2} \mathrm{SO}_{4}$ and the solvents were removed under reduced pressure. The crude product was purified by column chromatography (pentane) to give 3-methyl-2-phenyl-but-2-ene (112) $(0.26 \mathrm{~g}, 1.8 \mathrm{mmol}, 19 \%)$ as a colorless liquid. ${ }^{1} \mathrm{H}$ NMR $\left(\mathrm{CDCl}_{3}, 300 \mathrm{MHz}\right)^{28 \mathrm{~b}} \delta 7.33-7.11(\mathrm{~m}, 5 \mathrm{H}, \mathrm{Ar} \mathrm{H}), 1.96\left(\mathrm{~m}, 3 \mathrm{H}, \mathrm{CH}_{3}\right), 1.81\left(\mathrm{~s}, 3 \mathrm{H}, \mathrm{CH}_{3}\right), 1.59(\mathrm{q}, J=$ $\left.1.4 \mathrm{~Hz}, 3 \mathrm{H}, \mathrm{CH}_{3}\right)$.

(R)-(E)-But-2-enoic acid 4,4-dimethyl-2-oxo-tetrahydro-furan-3-yl ester (25)

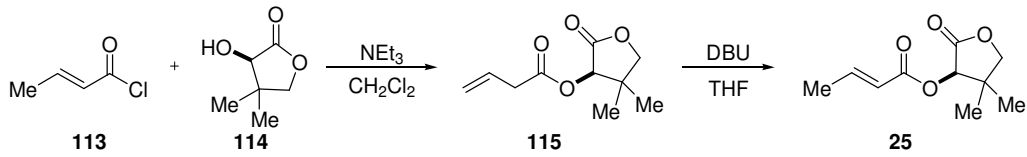

(R)-But-3-enoic acid 4,4-dimethyl-2-oxo-tetrahydro-furan-3-yl ester (115)

Triethylamine (distilled, $2.1 \mathrm{~mL}, 15 \mathrm{mmol}, 1.5$ equiv) was added to a solution of D-Pantolactone (114) $(1.30 \mathrm{~g}, 10.0 \mathrm{mmol}, 1.00$ equiv) in $\mathrm{CH}_{2} \mathrm{Cl}_{2}\left(17 \mathrm{~mL}\right.$ ) at $-20{ }^{\circ} \mathrm{C}$ under argon. Crotonyl chloride (113) (freshly distilled, $1.2 \mathrm{~mL}, 13 \mathrm{mmol}, 1.3$ equiv) was then added dropwise over $30 \mathrm{~min}$. After warming up to $23{ }^{\circ} \mathrm{C}$ over $4 \mathrm{~h}$, the reaction mixture was washed with $1 \mathrm{M} \mathrm{HCl}(2 \times 15 \mathrm{~mL})$, sat. $\mathrm{NaHCO}_{3}(2 \times 15 \mathrm{~mL})$ and water $(15 \mathrm{~mL})$, dried over $\mathrm{Na}_{2} \mathrm{SO}_{4}$ and the solvent was removed under reduced pressure. The crude product was purified by column chromatography (AcOEt/hexane 1:4) to give product $115(1.77 \mathrm{~g}, 8.93 \mathrm{mmol}, 89 \%)$ as an oil. $\mathrm{R}_{\mathrm{f}}$ (AcOEt/hexane 1:5) 0.20; ${ }^{1} \mathrm{H} \mathrm{NMR}\left(\mathrm{CDCl}_{3}, 300 \mathrm{MHz}\right) \delta$ 5.99-5.89 (m, 1H, alkene $\left.\mathrm{H}\right), 5.38\left(\mathrm{~s}, 1 \mathrm{H}, \mathrm{C}_{2} \mathrm{CHOH}\right), 5.27-5.20(\mathrm{~m}, 2 \mathrm{H}$, alkene $\mathrm{H}), 4.08-4.00\left(\mathrm{~m}, 2 \mathrm{H}, \mathrm{CH}_{2} \mathrm{O}\right), 3.27-3.24\left(\mathrm{~m}, 2 \mathrm{H}\right.$, alkene $\left.\mathrm{CH}_{2}\right), 1.21\left(\mathrm{~s}, 3 \mathrm{H}, \mathrm{CH}_{3}\right), 1.11\left(\mathrm{~s}, 3 \mathrm{H}, \mathrm{CH}_{3}\right) ;{ }^{13} \mathrm{C} \mathrm{NMR}\left(\mathrm{CDCl}_{3}, 75\right.$ 
MHz) $\delta 172.0,170.1,129.1,119.2,76.1,75.1,40.2,38.5,23.1,19.9 ;$ IR v $3085(w), 2970(\mathrm{~m}), 2936$ (w), $2912(\mathrm{w}), 2880(\mathrm{w})$, 1792 (s), 1750 (s), 1644 (w), 1467 (m), 1426 (w), 1402 (m), 1377 (m), 1370 (m), 1349 (w), $1325(\mathrm{~m}), 1297$ (m), 1249 (m), 1200 (m), 1153 (s), 1106 (s), 1081 (s), 1033 (m), 1014 (s), 997 (s), 927 (m), 813 (w), 708 (w), 558 (w); HRMS (EI) calcd for C ${ }_{10} \mathrm{H}_{14} \mathrm{O}_{4}{ }^{+}$ (M): 198.0892, found 198.0891; Anal. calcd for $\mathrm{C}_{10} \mathrm{H}_{14} \mathrm{O}_{4}$ : C, 60.59; H, 7.12. Found: C, 60.82; H, 7.10.

$(\boldsymbol{R})$-(E)-But-2-enoic acid 4,4-dimethyl-2-oxo-tetrahydro-furan-3-yl ester (25)

DBU (1.0 mL, $6.2 \mathrm{mmol}, 1.3$ equiv) was added dropwise to a solution of $\mathbf{1 1 5}$ (1.0 g, $5.0 \mathrm{mmol}, 1.0$ equiv) in THF (5 mL) at 0 ${ }^{\circ} \mathrm{C}$ under argon. The reaction mixture was stirred $30 \mathrm{~min}$ at $0{ }^{\circ} \mathrm{C}$ and $5 \mathrm{~h}$ at $23{ }^{\circ} \mathrm{C}$, diluted with $\mathrm{Et}_{2} \mathrm{O}(20 \mathrm{~mL})$, washed with $3 \mathrm{M}$ $\mathrm{HCl}(3 \times 15 \mathrm{~mL})$ and the water layers were extracted with $\mathrm{Et}_{2} \mathrm{O}(3 \times 15 \mathrm{~mL})$. The combined organic layers were washed with $10 \%$ $\mathrm{NaOH}(2 \times 15 \mathrm{~mL}), 2 \mathrm{M} \mathrm{HCl}(2 \times 15 \mathrm{~mL})$ and water $(15 \mathrm{~mL})$, dried over $\mathrm{Na}_{2} \mathrm{SO}_{4}$ and the solvent was removed under reduced pressure to give product $25(0.84 \mathrm{~g}, 4.2 \mathrm{mmol}, 84 \%)$ as a yellow oil. $\mathrm{R}_{\mathrm{f}}($ AcOEt/hexane $1: 5) 0.20 ;{ }^{1} \mathrm{H} \mathrm{NMR}\left(\mathrm{CDCl}{ }_{3}, 300 \mathrm{MHz}\right) \delta$ $7.12(\mathrm{dq}, J=15.6,6.8 \mathrm{hz}, 1 \mathrm{H}$, alkene $\mathrm{H}), 5.96\left(\mathrm{dq}, J=15.6,1.9 \mathrm{~Hz}, 1 \mathrm{H}\right.$, alkene H), $5.43\left(\mathrm{~s}, 1 \mathrm{H}, \mathrm{C}_{2} \mathrm{CHO}\right), 4.08(\mathrm{~d}, J=9.0 \mathrm{~Hz}, 1 \mathrm{H}$, $\left.\mathrm{CH}_{2} \mathrm{O}\right), 4.03\left(\mathrm{~d}, J=9.0 \mathrm{~Hz}, 1 \mathrm{H}, \mathrm{CH}_{2} \mathrm{O}\right), 1.93\left(\mathrm{dd}, J=6.8,1.9 \mathrm{~Hz}, 3 \mathrm{H}, \mathrm{CH}_{3}\right), 1.22\left(\mathrm{~s}, 3 \mathrm{H}, \mathrm{CH}_{3}\right), 1.13\left(\mathrm{~s}, 3 \mathrm{H}, \mathrm{CH}_{3}\right) ;{ }^{13} \mathrm{C} \mathrm{NMR}^{\mathrm{N}}$ $\left(\mathrm{CDCl}_{3}, 75 \mathrm{MHz}\right) \delta 172.4,164.8,147.1,121.1,76.2,74.7,40.4,23.1,20.0,18.3 ; \mathrm{IR} v 2970(\mathrm{~m}), 2936(\mathrm{w}), 2915(\mathrm{w}), 2879(\mathrm{w})$, 1794 (s), 1731 (s), 1656 (m), 1466 (w), 1444 (m), 1401 (w), 1377 (m), 1344 (w), 1294 (m), 1256 (s), 1171 (s), 1107 (s), 1087 (s), 1032 (m), $1014(\mathrm{~m}), 997(\mathrm{~m}), 970(\mathrm{~m}), 915$ (w), $894(\mathrm{w}), 836(\mathrm{w}), 732(\mathrm{w}), 685(\mathrm{w}), 650$ (w), $562(\mathrm{w}), 516(\mathrm{w})$; HRMS (EI) calcd for $\mathrm{C}_{10} \mathrm{H}_{14} \mathrm{O}_{4}^{+}(\mathrm{M})$ : 198.0892, found 198.0891; Anal. calcd for $\mathrm{C}_{10} \mathrm{H}_{14} \mathrm{O}_{4}$ : C, 60.59; H, 7.12. Found: C, 60.76; H, 7.17.

\subsection{The Co- and Mn- Catalyzed Hydrohydrazination of Olefins}

\subsubsection{General Procedures}

General Procedure for the Co-Catalyzed Hydrohydrazination of Olefins (GP1)

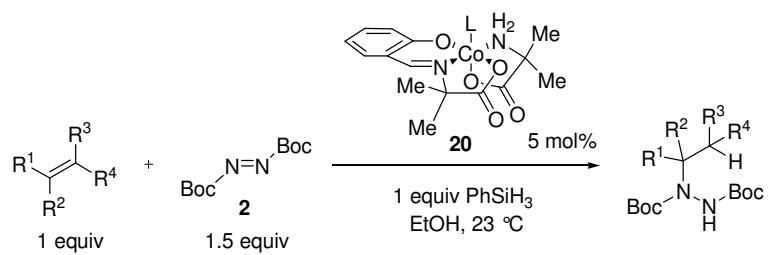

The Co catalyst 20 (10 mg, $0.025 \mathrm{mmol} 0.050$ equiv) was dissolved in ethanol $(2.5 \mathrm{~mL})$ at $23{ }^{\circ} \mathrm{C}$ under argon. The olefin $(0.50$ mmol, 1.0 equiv) and phenylsilane $(65 \mu \mathrm{L}, 0.52 \mathrm{mmol}, 1.0$ equiv) were added to the brown-red solution, followed by di-tert-butyl azodicarboxylate (2) $\left(0.17 \mathrm{~g}, 0.75 \mathrm{mmol}, 1.5\right.$ equiv) in one portion. The resulting solution was stirred at $23{ }^{\circ} \mathrm{C}$ and monitored by TLC (AcOEt/hexane 1:5). After completion the reaction was quenched with $\mathrm{H}_{2} \mathrm{O}(1 \mathrm{~mL})$, brine $(5 \mathrm{~mL})$ was added and the reaction mixture was extracted with AcOEt $(3 \times 10 \mathrm{~mL})$. The combined organic layers were dried over $\mathrm{Na}_{2} \mathrm{SO}_{4}$, filtered and the solvents were removed under reduced pressure. The isolated product was purified by column chromatography.

General Procedure for the Co-Catalyzed Hydrohydrazination of Polymerization-Sensitive Olefins (GP2)

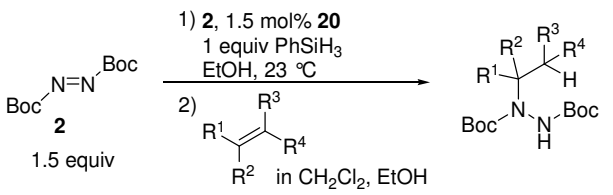

The Co catalyst 20 (3.0 mg, $0.0075 \mathrm{mmol}, 0.016$ equiv, in the case of vinyl heterocycles: $5.0 \mathrm{mg}, 0.012 \mathrm{mmol}, 0.025$ equiv) was dissolved in ethanol $(2.5 \mathrm{~mL})$ at $23{ }^{\circ} \mathrm{C}$ under argon. Phenylsilane $(65 \mu \mathrm{L}, 0.52 \mathrm{mmol}, 1.0$ equiv) and di-tert-butyl azodicarboxylate (2) $(0.17 \mathrm{~g}, 0.75 \mathrm{mmol}, 1.5$ equiv) were added to the brown-red solution, followed by a solution of olefin ( 0.50 mmol, 1.0 equiv) in ethanol $(0.5 \mathrm{~mL}$, in the case of vinyl heterocycles: $1.0 \mathrm{~mL})$ and $\mathrm{CH}_{2} \mathrm{Cl}_{2}(0.5 \mathrm{~mL}$, in the case of vinyl heterocycles: $1.0 \mathrm{~mL}$ ). The resulting solution was stirred at $23{ }^{\circ} \mathrm{C}$ and monitored by TLC (AcOEt/hexane 1:5). After completion the reaction was quenched with $\mathrm{H}_{2} \mathrm{O}(1 \mathrm{~mL})$, brine $(5 \mathrm{~mL})$ was added and the reaction mixture was extracted with AcOEt $(3 \times 10$ $\mathrm{mL})$. The combined organic layers were dried over $\mathrm{Na}_{2} \mathrm{SO}_{4}$, filtered and the solvents were removed under reduced pressure. The isolated product was purified by column chromatography.

General Procedure for the Mn-Catalyzed Hydrohydrazination of Olefins (GP3)

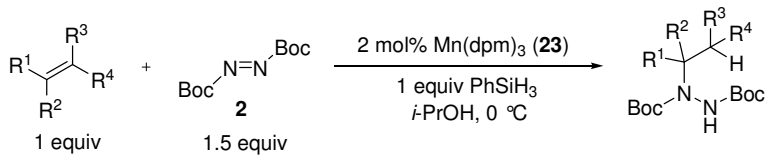

$\mathrm{Mn}(\mathrm{dpm})_{3}(\mathbf{2 3})\left(6 \mathrm{mg}, 0.01 \mathrm{mmol}, 0.02\right.$ equiv) was dissolved in isopropanol $(2.5 \mathrm{~mL})$ at $23{ }^{\circ} \mathrm{C}$ under argon and the dark browngreen solution was cooled to $0{ }^{\circ} \mathrm{C}$. The olefin $(0.50 \mathrm{mmol}, 1.0$ equiv) and phenylsilane $(65 \mu \mathrm{L}, 0.52 \mathrm{mmol}, 1.0$ equiv) were added, followed by di-tert-butyl azodicarboxylate (2) $(0.17 \mathrm{~g}, 0.75 \mathrm{mmol}, 1.5 \mathrm{equiv})$ in one portion. The resulting suspension was stirred at $0{ }^{\circ} \mathrm{C}$ and monitored by TLC (AcOEt/hexane 1:5). After completion (color change to yellow) the reaction was quenched with $\mathrm{H}_{2} \mathrm{O}(1 \mathrm{~mL})$, brine $(5 \mathrm{~mL})$ was added and the reaction mixture was extracted with AcOEt $(3 \times 10 \mathrm{~mL})$. The combined organic 
layers were dried over $\mathrm{Na}_{2} \mathrm{SO}_{4}$, filtered and the solvents were removed under reduced pressure. The isolated product was purified by column chromatography.

General Procedure for the Mn-Catalyzed Hydrohydrazination of Polymerization-Sensitive Olefins (GP4)

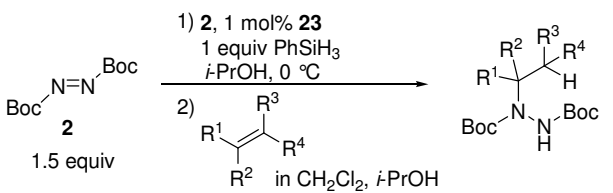

The Mn catalyst 23 (3 mg, $0.005 \mathrm{mmol}, 0.01$ equiv) was dissolved in isopropanol $(2.5 \mathrm{~mL})$ at $23{ }^{\circ} \mathrm{C}$ under argon and the dark brown-green solution was cooled to $0{ }^{\circ} \mathrm{C}$. Phenylsilane $(65 \mu \mathrm{L}, 0.52 \mathrm{mmol}, 1.0$ equiv) and di-tert-butyl azodicarboxylate $(2)(0.17$ $\mathrm{g}, 0.75 \mathrm{mmol}, 1.5$ equiv) were added, followed by a solution of olefin in isopropanol $(1 \mathrm{~mL})$ and $\mathrm{CH}_{2} \mathrm{Cl}_{2}(1 \mathrm{~mL})$. The resulting solution was stirred at $0{ }^{\circ} \mathrm{C}$ and monitored by TLC (AcOEt/hexane 1:5). After completion (color change to yellow) the reaction was quenched with $\mathrm{H}_{2} \mathrm{O}(1 \mathrm{~mL})$, brine $(5 \mathrm{~mL})$ was added and the reaction mixture was extracted with AcOEt $(3 \times 10 \mathrm{~mL})$. The combined organic layers were dried over $\mathrm{Na}_{2} \mathrm{SO}_{4}$, filtered and the solvents were removed under reduced pressure. The isolated product was purified by column chromatography.

\subsubsection{Hydration of enyne 5}

4-Methoxy-benzoic acid 4-hydroxy-1,1-dimethyl-pent-2-ynyl ester (6)

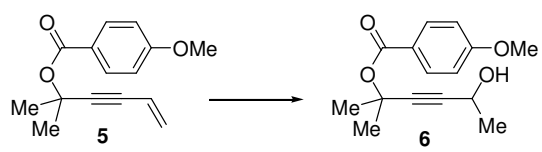

\section{With Co catalyst from ligand 7}

The catalyst (18 mg, $0.036 \mathrm{mmol}, 0.13$ equiv) was dissolved in $\mathrm{CH}_{2} \mathrm{Cl}_{2}(3 \mathrm{~mL})$ at $23{ }^{\circ} \mathrm{C}$ under $\mathrm{O}_{2}(1$ atm, balloon). Enyne 5 (69 $\mathrm{mg}, 0.28 \mathrm{mmol}, 1.0$ equiv) and $\mathrm{PhSiH}_{3}(75 \mu \mathrm{L}, 0.60 \mathrm{mmol}, 2.2$ equiv) were added and the resulting green-brown solution was stirred at $23{ }^{\circ} \mathrm{C}$ and the reaction was monitored by TLC (AcOEt/hexane 1:2). After completion (9 h), the solvent was removed under reduced pressure, the residues were dissolved in $\mathrm{MeOH}(3 \mathrm{~mL})$, sat. $\mathrm{Na}_{2} \mathrm{~S}_{2} \mathrm{O}_{3}(1 \mathrm{~mL})$ was added, the mixture stirred vigorously for $30 \mathrm{~min}$ and quenched with sat. $\mathrm{NaCl}(5 \mathrm{~mL})$. The reaction mixture was then extracted with $\mathrm{AcOEt}(3 \times 10 \mathrm{~mL})$, the organic layers were dried over $\mathrm{Na}_{2} \mathrm{SO}_{4}$ and the solvent removed under reduced pressure. The crude product was purified by column chromatography (AcOEt/hexane 1:2) to afford propargylic alcohol 6 (57 mg, $0.22 \mathrm{mmol}, 77 \%$ ) as a colorless oil.

\section{With Co catalyst 20}

Catalyst 20 ( $0.8 \mathrm{mg}, 2 \mu \mathrm{mol}, 0.01$ equiv) was dissolved in ethanol $(2 \mathrm{~mL})$ at $23{ }^{\circ} \mathrm{C}$ under $\mathrm{O}_{2}$ ( $1 \mathrm{~atm}$, balloon). Enyne 5 (48 mg, $0.20 \mathrm{mmol}, 1.0$ equiv) and $\mathrm{PhSiH}_{3}(50 \mu \mathrm{L}, 0.40 \mathrm{mmol}, 2.0$ equiv) were added and the resulting dark brown red solution was stirred at $23{ }^{\circ} \mathrm{C}$ and the reaction was monitored by TLC (AcOEt/hexane 1:2). After completion (45 min), the solvent was removed under reduced pressure, the residues were dissolved in $\mathrm{MeOH}(3 \mathrm{~mL})$, sat. $\mathrm{Na}_{2} \mathrm{~S}_{2} \mathrm{O}_{3}(1 \mathrm{~mL})$ was added, the mixture stirred vigorously for $30 \mathrm{~min}$ and quenched with sat. $\mathrm{NaCl}(5 \mathrm{~mL})$. The reaction mixture was then extracted with $\mathrm{AcOEt}(3 \times 10 \mathrm{~mL})$, the organic layers were dried over $\mathrm{Na}_{2} \mathrm{SO}_{4}$ and the solvent removed under reduced pressure. The crude product was purified by column chromatography (AcOEt/hexane 1:2) to afford propargylic alcohol 6 (47 mg, $0.18 \mathrm{mmol}, 91 \%$ ) as a colorless oil. $\mathrm{R}_{\mathrm{f}}$ (AcOEt/hexane 1:2) 0.30; ${ }^{1} \mathrm{H} \mathrm{NMR}\left(\mathrm{CDCl}_{3}, 300 \mathrm{MHz}\right) \delta 7.94(\mathrm{dd}, J=6.9,2.2 \mathrm{~Hz}, 2 \mathrm{H}, \mathrm{Ar} \mathrm{H}), 6.89(\mathrm{dd}, J=6.9,1.9 \mathrm{~Hz}, 2 \mathrm{H}, \mathrm{Ar}$ $\mathrm{H}), 4.56\left(\mathrm{q}, J=6.5 \mathrm{~Hz}, 1 \mathrm{H}, \mathrm{CHOHCH}_{3}\right), 3.84\left(\mathrm{~s}, 3 \mathrm{H}, \mathrm{OCH}_{3}\right), 2.77(\mathrm{bs}, \mathrm{OH}), 1.76\left(\mathrm{~s}, 6 \mathrm{H},\left(\mathrm{CH}_{3}\right)_{2} \mathrm{COC}\right), 1.43(\mathrm{~d}, J=6.5 \mathrm{~Hz}, 3 \mathrm{H}$, $\left.\mathrm{CHOHCH}_{3}\right) ;{ }^{13} \mathrm{C} \mathrm{NMR}\left(\mathrm{CDCl}_{3}, 75 \mathrm{MHz}\right) \delta 164.5,163.1,131.5,123.2,113.4,86.2,84.9,72.2,58.2,55.4,29.2,24.1 ; \mathrm{IR} v 3448$ (m), $2985(\mathrm{~m}), 2936(\mathrm{~m}), 2841(\mathrm{w}), 1717(\mathrm{~s}), 1607(\mathrm{~s}), 1582(\mathrm{w}), 1511(\mathrm{~m}), 1465(\mathrm{~m}), 1421(\mathrm{~m}), 1383(\mathrm{w}), 1365(\mathrm{~m}), 1318(\mathrm{~m})$, $1286(\mathrm{~s}), 1258$ (s), $1171(\mathrm{~m}), 1140$ (s), 1100 (s), $1029(\mathrm{~m}), 983(\mathrm{w}), 925$ (m), $848(\mathrm{~m}), 771(\mathrm{~m}), 698(\mathrm{~m}), 622(\mathrm{w})$; HRMS(EI) calcd for $\mathrm{C}_{15} \mathrm{H}_{18} \mathrm{O}_{4}^{+}(\mathrm{M}) 262.1205$, found 262.1195 .

\subsubsection{Hydrohydrazination Products}

$N$-(3-Phenyl-1-methyl-propyl)- $N$ '-(tert-butoxycarbonyl)hydrazinecarboxylic acid tert-butyl ester (3) and $N$-(4-Phenylbutyl)- $N$ '-(tert-butoxycarbonyl)hydrazinecarboxylic acid tert-butyl ester (24)

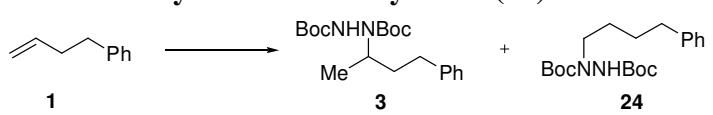

Following GP1 Hydrohydrazination product $3(155 \mathrm{mg}, 0.425 \mathrm{mmol}, 85 \%)$ was obtained as a colorless solid with 4 phenylbutene (1) $(75 \mu \mathrm{L}, 0.50 \mathrm{mmol}, 1.0$ equiv) in $4 \mathrm{~h}$ with 5 mol\% catalyst 20 after purification by column chromatography (AcOEt/hexane 1:10). Scale up The cobalt catalyst $20\left(60 \mathrm{mg}, 0.12 \mathrm{mmol} 0.025\right.$ equiv) was dissolved in ethanol $(20 \mathrm{~mL})$ at $23{ }^{\circ} \mathrm{C}$ under argon. 4-Phenylbutene (1) $(675 \mathrm{mg}, 0.510 \mathrm{mmol} 1.00$ equiv) and phenylsilane (0.65 mL, $5.2 \mathrm{mmol}, 1.0$ equiv) were added to the brown-red solution, followed by di-tert-butyl azodicarboxylate (2) (1.72 g, $7.47 \mathrm{mmol}, 1.50$ equiv) portionswise. The resulting solution was stirred at $23{ }^{\circ} \mathrm{C}$ and monitored by TLC (AcOEt/hexane 1:5). After completion $(5 \mathrm{~h}$ ) the reaction mixture 
was concentrated under reduced pressure to about $3 \mathrm{~mL}$, quenched with $\mathrm{H}_{2} \mathrm{O}(3 \mathrm{~mL})$, brine $(20 \mathrm{~mL})$ was added and the reaction mixture was extracted with AcOEt $(3 \times 50 \mathrm{~mL})$. The combined organic layers were dried over $\mathrm{Na}_{2} \mathrm{SO}_{4}$, filtered and the solvents were removed under reduced pressure. The isolated product was purified by column chromatography (AcOEt/hexane $1: 10)$ to afford $3(1.75 \mathrm{~g}, 4.80 \mathrm{mmol}, 94 \%)$ as a colorless solid together with recovered 4-phenylbutene (1) (38 mg, $0.28 \mathrm{mmol}, 5 \%)$. Following GP1, but using TMDSO Hydrohydrazination product $3(156 \mathrm{mg}, 0.428 \mathrm{mmol}, 86 \%)$ was obtained as a colorless solid with 4-phenylbutene (1) $(75 \mu \mathrm{L}, 0.50 \mathrm{mmol}, 1.0$ equiv) and TMDSO (0.13 mL, $0.75 \mathrm{mmol}, 1.5$ equiv) in $4 \mathrm{~h}$ with $5 \mathrm{~mol} \%$ catalyst 20 after purification by column chromatography (AcOEt/hexane 1:10). Following GP3 Hydrohydrazination product 3 (140 mg, $0.384 \mathrm{mmol}, 76 \%)$ together with the primary hydrazide $24(34 \mathrm{mg}, 0.093 \mathrm{mmol}, 18 \%$, total $94 \%)$ were obtained as colorless solids with 4-phenylbutene (1) $(75 \mu \mathrm{L}, 0.50 \mathrm{mmol}, 1.0 \mathrm{eq})$ in $2.5 \mathrm{~h}$ with $2 \mathrm{~mol} \%$ catalyst 23 after purification by column chromatography (AcOEt/hexane 1:10). Following GP3, but with PMHS Hydrohydrazination product 3 (142 mg, 0.390 mmol, $78 \%)$ together with the primary hydrazide $24(20 \mathrm{mg}, 0.056 \mathrm{mmol}, 11 \%$, total $89 \%)$ were obtained as colorless solids with 4 phenylbutene (1) $(75 \mu \mathrm{L}, 0.50 \mathrm{mmol}, 1.0 \mathrm{eq})$ and PMHS $\left(0.10 \mathrm{~mL}, 1.6 \mathrm{H}\right.$ equiv) in $20 \mathrm{~h}$ at $23{ }^{\circ} \mathrm{C}$ with 2 mol\% catalyst 23 after purification by column chromatography (AcOEt/hexane 1:10).

$\boldsymbol{N}$-(3-Phenyl-1-methyl-propyl)- $N$ '-(tert-butoxycarbonyl)hydrazinecarboxylic acid tert-butyl ester (3)

$\mathrm{R}_{\mathrm{f}}\left(\mathrm{AcOEt} / \mathrm{h}\right.$ exane 1:5) 0.35; Mp 125-127 ${ }^{\circ} \mathrm{C} ;{ }^{1} \mathrm{H} \mathrm{NMR}\left(\mathrm{CDCl}_{3}, 300 \mathrm{MHz}, 52{ }^{\circ} \mathrm{C}\right) \delta$ 7.29-7.14 (m, 5H, Ar H), $5.86(\mathrm{br} \mathrm{s}, 1 \mathrm{H}$, $\mathrm{NH}), 4.25$ (br s, $1 \mathrm{H}, \mathrm{CHN}), 2.63\left(\mathrm{~m}, 2 \mathrm{H}, \mathrm{PhCH}_{2}\right), 1.90\left(\mathrm{~m}, 1 \mathrm{H}, \mathrm{CH}_{2} \mathrm{CHN}\right), 1.65\left(\mathrm{~m}, 1 \mathrm{H}, \mathrm{CH}_{2} \mathrm{CHN}\right), 1.48\left(\mathrm{~s}, 9 \mathrm{H}, \mathrm{CCH}_{3}\right), 1.47(\mathrm{~s}$,

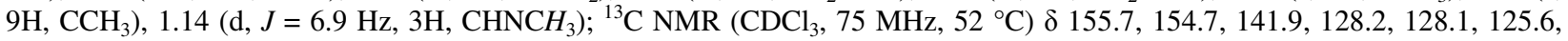
80.8, 53.1, 35.8, 32.9, 28.3, 28.2, 18.2; IR (KBr) v 3290 (s), 3070 (w), 2977 (s), 2927 (m), 1744 (s), 1665 (s), 1514 (s), 1411 (s), 1369 (s), 1346 (s), 1245 (s), 1153 (s), 1116 (s), 1074 (m), 1004 (m), 909 (m), 898 (m), 858 (m), 784 (m), 747 (s), 702 (s), 592 (m); HRMS (ESI) calcd for $\mathrm{C}_{20} \mathrm{H}_{32} \mathrm{~N}_{2} \mathrm{O}_{4} \mathrm{Na}^{+}(\mathrm{M}+\mathrm{Na})$ : 387.2254, found 387.2255; Anal. calcd for $\mathrm{C}_{20} \mathrm{H}_{32} \mathrm{~N}_{2} \mathrm{O}_{4}$ : C, 65.91; $\mathrm{H}, 8.85$; N, 7.69. Found: C, 65.69; H, 8.77; N, 7.60.

\section{$\boldsymbol{N}$-(4-Phenyl-butyl)- $\boldsymbol{N}^{\prime}$-(tert-butoxycarbonyl)hydrazinecarboxylic acid tert-butyl ester (24)}

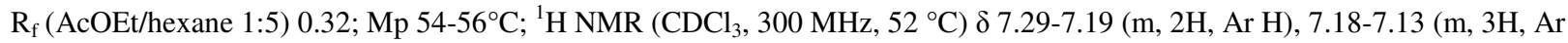
$\mathrm{H}), 6.21($ br s, $1 \mathrm{H}, \mathrm{NH}), 3.46\left(\mathrm{t}, J=6.5 \mathrm{~Hz}, 2 \mathrm{H}, \mathrm{CH}_{2} \mathrm{~N}\right), 2.64\left(\mathrm{t}, J=7.2 \mathrm{~Hz}, 2 \mathrm{H}, \mathrm{CH}_{2} \mathrm{Ph}\right), 1.70-1.53\left(\mathrm{~m}, 4 \mathrm{H}, \mathrm{CH}_{2}\right), 1.47(\mathrm{~s}, 9 \mathrm{H}$, $\left.\mathrm{CCH}_{3}\right), 1.46\left(\mathrm{~s}, 9 \mathrm{H}, \mathrm{CCH}_{3}\right) ;{ }^{13} \mathrm{C} \mathrm{NMR}\left(\mathrm{CDCl}_{3}, 75 \mathrm{MHz}, 52{ }^{\circ} \mathrm{C}\right) \delta 155.2,142.2,128.3,128.2,125.6,81.0,80.9,49.8,35.6,28.5$, 28.3, 28.3, 27.2; IR v 3317 (m), 3063 (w), 3026 (w), 2978 (s), 2933 (s), $2863(\mathrm{w}), 1706$ (s), $1604(\mathrm{w}), 1496$ (s), 1479 (s), 1454 (s), 1393 (s), 1367 (s), 1254 (s), 1153 (s), 1090 (w), 1074 (w), 1053 (w), 1017 (w), 932 (w), $856(w), 750$ (m), 700 (m), 584 (w), 494 (w); MS 365.1 (M+H); Anal. calcd for $\mathrm{C}_{20} \mathrm{H}_{32} \mathrm{~N}_{2} \mathrm{O}_{4}$ : C, 65.91; H, 8.85; N, 7.69. Found: C, 65.91; H, 8.79; N, 7.59.

$N$-(3-Phenyl-1-methyl-propyl)- $N$ '-(ethoxycarbonyl)hydrazinecarboxylic acid ethyl ester (116)

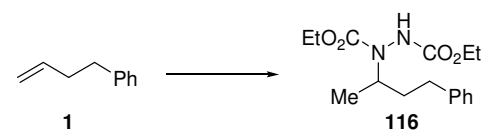

Following GP1, but using DEAD Hydrohydrazination product 116 (51 mg, $0.17 \mathrm{mmol}$, 34\%) was obtained as a colorless viscous oil with 4-phenylbutene (1) $(75 \mu \mathrm{L}, 0.50 \mathrm{mmol}, 1.0$ equiv) and diethyl azodicarboxylate $(0.12 \mathrm{~mL}, 0.75 \mathrm{mmol}, 1.5 \mathrm{equiv})$ in $1.5 \mathrm{~h}$ with $5 \mathrm{~mol} \%$ catalyst 20 after purification by column chromatography (AcOEt/hexane 1:5). $\mathrm{R}_{\mathrm{f}}($ AcOEt/hexane 1:5) 0.20; ${ }^{1} \mathrm{H}$ NMR $\left(\mathrm{CDCl}_{3}, 300 \mathrm{MHz}, 52{ }^{\circ} \mathrm{C}\right) \delta$ 7.36-7.21 (m, 5H, Ar H), 6.19 (br s, 1H, NH), 4.35 (br s, 1H, CHN), 4.30-4.21 (m, 4H, $\left.\mathrm{OCH}_{2}\right), 2.74\left(\mathrm{~m}, 2 \mathrm{H}, \mathrm{CH}_{2} \mathrm{Ph}\right), 1.99\left(\mathrm{~m}, 1 \mathrm{H}, \mathrm{CH}_{2} \mathrm{CHN}\right), 1.75\left(\mathrm{~m}, 1 \mathrm{H}, \mathrm{CH}_{2} \mathrm{CHN}\right), 1.33\left(\mathrm{~m}, 6 \mathrm{H}, \mathrm{OCH}_{2} \mathrm{CH}_{3}\right), 1.23(\mathrm{~d}, J=6.5 \mathrm{~Hz}, 3 \mathrm{H}$, $\left.\mathrm{CH}_{3} \mathrm{CHN}\right) ;{ }^{13} \mathrm{C} \mathrm{NMR}\left(\mathrm{CDCl}_{3}, 75 \mathrm{MHz}, 52{ }^{\circ} \mathrm{C}\right) \delta 156.9,155.9,141.8,128.2,125.7,109.9,62.3,62.0,53.8,35.7,33.0,18.2,14.6$, 14.5; IR v $3289(\mathrm{~m}), 3085(\mathrm{w}), 3062(\mathrm{w}), 3027(\mathrm{w}), 2981(\mathrm{~m}), 2934(\mathrm{~m}), 2871(\mathrm{~m}), 1755(\mathrm{~s}), 1711(\mathrm{~s}), 1604(\mathrm{w}), 1519(\mathrm{~m}), 1496$ $(\mathrm{m}), 1454(\mathrm{~m}), 1415(\mathrm{~s}), 1378(\mathrm{~m}), 1327(\mathrm{~m}), 1228(\mathrm{~s}), 1174(\mathrm{~m}), 1114(\mathrm{~m}), 1097(\mathrm{~m}), 1061(\mathrm{~m}), 915(\mathrm{w}), 850(\mathrm{w}), 760(\mathrm{~m}), 701$ (m), 621 (w), $594(\mathrm{w}), 488(\mathrm{w})$; HRMS (ESI) calcd for $\mathrm{C}_{16} \mathrm{H}_{24} \mathrm{~N}_{2} \mathrm{O}_{4} \mathrm{Na}^{+}$(M+Na) 331.1628, found 331.1630; Anal. calcd for $\mathrm{C}_{16} \mathrm{H}_{24} \mathrm{~N}_{2} \mathrm{O}_{4}$ : C, 62.32; H, 7.84; N, 9.08. Found: C, 62.30; H, 7.75; N, 8.90.

$N$-(1-Phenyl-ethyl)- $N$ '-(tert-butoxycarbonyl)hydrazinecarboxylic acid tert-butyl ester (118)

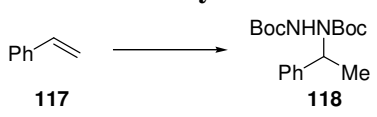

Following GP2 Hydrohydrazination product 118 (145 mg, $0.431 \mathrm{mmol}, 86 \%)$ was obtained as a colorless solid with styrene (117) (freshly filtered through neutral $\mathrm{Al}_{2} \mathrm{O}_{3}, 57 \mu \mathrm{L}, 0.50 \mathrm{mmol}, 1.0$ equiv) in $3 \mathrm{~h}$ with 5 mol\% catalyst 20 after purification by column chromatography (AcOEt/hexane 1:10). $\mathrm{R}_{\mathrm{f}}(\mathrm{AcOEt} /$ hexane $1: 3) 0.43 ; \mathrm{Mp} 87-88{ }^{\circ} \mathrm{C} ;{ }^{1} \mathrm{H} \mathrm{NMR}\left(\mathrm{CDCl}_{3}, 300 \mathrm{MHz}, 52{ }^{\circ} \mathrm{C}\right) \delta$ 7.34-7.20 (m, 5H, Ar H), 5.95 (br s, 1H, NH), 5.43 (br m, 1H, CHN), 1.52 (d, J = 7.2 Hz, 3H, CHCH $), 1.46\left(\mathrm{~s}, 9 \mathrm{H}, \mathrm{CCH}_{3}\right), 1.43$ (br s, 9H, $\left.\mathrm{CCH}_{3}\right) ;{ }^{13} \mathrm{C} \mathrm{NMR}\left(\mathrm{CDCl}_{3}, 75 \mathrm{MHz}, 52{ }^{\circ} \mathrm{C}\right) \delta 155.6,154.6,141.2,128.2,127.2,81.1,80.8,55.8,28.3,28.2,17.0 ;$ IR $\mathrm{v}$ $3311(\mathrm{~m}), 3088$ (w), 3064 (w), 3032 (w), 2979 (m), 2934 (m), 1702 (s), $1495(\mathrm{~m}), 1479(\mathrm{~m}), 1455$ (m), 1393 (s), 1367 (s), 1316 (s), $1251(\mathrm{~s}), 1168(\mathrm{~s}), 1048(\mathrm{~m}), 1030(\mathrm{~m}), 990(\mathrm{w}), 912(\mathrm{w}), 860(\mathrm{w}), 760(\mathrm{~m}), 700(\mathrm{~m}), 580(\mathrm{w})$; HRMS (ESI) calcd for $\mathrm{C}_{18} \mathrm{H}_{28} \mathrm{~N}_{2} \mathrm{O}_{4} \mathrm{Na}^{+}(\mathrm{M}+\mathrm{Na}): 359.1941$, found 359.1945; Anal. calcd for $\mathrm{C}_{18} \mathrm{H}_{28} \mathrm{~N}_{2} \mathrm{O}_{4}: \mathrm{C}, 64.26 ; \mathrm{H}, 8.39 ; \mathrm{N}, 8.33$. Found: C, 63.99; $\mathrm{H}$, $8.36 ; \mathrm{N}, 8.29$.

$N$-(2-Hydroxy-1-methyl-ethyl)-N'-(tert-butoxycarbonyl)hydrazinecarboxylic acid tert-butyl ester (120)

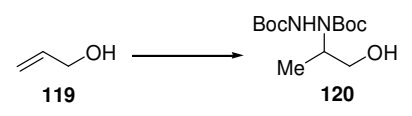


Following GP1 Hydrohydrazination product $120(113 \mathrm{mg}, 0.390 \mathrm{mmol}, 78 \%)$ was obtained as a colorless solid with allyl alcohol (119) $(34 \mu \mathrm{L}, 0.50 \mathrm{mmol}, 1.0$ equiv) in $3 \mathrm{~h}$ with $5 \mathrm{~mol} \%$ catalyst 20 after purification by column chromatography (AcOEt/hexane 1:2). $\mathrm{R}_{\mathrm{f}}$ (AcOEt/hexane 1:2) 0.40; Mp 119-120 ${ }^{\circ} \mathrm{C} ;{ }^{1} \mathrm{H} \mathrm{NMR}\left(\mathrm{CDCl}_{3}, 300 \mathrm{MHz}, 52{ }^{\circ} \mathrm{C}\right) \delta 6.26(\mathrm{br} \mathrm{s}, 1 \mathrm{H}, \mathrm{NH})$, 4.35 (br s, 1H, CHN), 4.08 (br s, $1 \mathrm{H}, \mathrm{OH}), 3.42\left(\mathrm{~m}, 2 \mathrm{H}, \mathrm{CH}_{2}\right), 1.46\left(\mathrm{~s}, 9 \mathrm{H}, \mathrm{CCH}_{3}\right), 1.44\left(\mathrm{~s}, 9 \mathrm{H}, \mathrm{CCH}_{3}\right), 0.97(\mathrm{~d}, J=6.9 \mathrm{~Hz}, 3 \mathrm{H}$, $\left.\mathrm{CHNCH}_{3}\right) ;{ }^{13} \mathrm{C} \mathrm{NMR}\left(\mathrm{CDCl}_{3}, 75 \mathrm{MHz}, 52{ }^{\circ} \mathrm{C}\right) \delta 157.9,155.3,82.0,81.5,63.5,55.3,28.2,28.1,13.6$; IR v $3358(\mathrm{~m}), 3234(\mathrm{~m})$, $2978(\mathrm{~m}), 2935$ (m), 2876 (w), 1713 (s), 1511 (m), 1460 (m), 1395 (s), 1368 (s), 1338 (s), 1292 (s), 1259 (s), 1156 (s), $1114(\mathrm{~s})$, 1061 (s), 1013 (m), 898 (w), 858 (m), 763 (m), 698 (w), 625 (w), 538 (w); MS (ESI) 291.2 (M+H), 313.1 (M+Na); Anal. calcd for $\mathrm{C}_{13} \mathrm{H}_{26} \mathrm{~N}_{2} \mathrm{O}_{5}$ : C, 53.78; H, 9.03; N, 9.65. Found: C, 53.92; H, 8.92; N, 9.52.

$N$-(2-Hydroxy-1-methyl-propyl)- $N$ '-(tert-butoxycarbonyl)hydrazinecarboxylic acid tert-butyl ester (122)

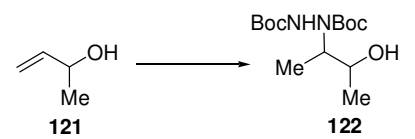

Following GP1 Hydrohydrazination product 122 (1. diastereoisomer: $57 \mathrm{mg}, 0.19 \mathrm{mmol}, 38 \%$; 2. diastereoisomer: $55 \mathrm{mg}$, 0.18 mmol, 36\%) was obtained as a colorless solid with but-3-en-2-ol (121) $(43 \mu \mathrm{L}, 0.50 \mathrm{mmol}, 1.0$ equiv) in $5 \mathrm{~h}$ with $5 \mathrm{~mol} \%$ catalyst 20 after purification by column chromatography (AcOEt/hexane 1:5-1:3). Diastereoisomer $1 \mathrm{R}_{\mathrm{f}}($ AcOEt/hexane 1:2) 0.33; Mp 111-113 ${ }^{\circ} \mathrm{C} ;{ }^{1} \mathrm{H}$ NMR $\left(\mathrm{CDCl}_{3}, 300 \mathrm{MHz}, 52{ }^{\circ} \mathrm{C}\right) \delta 6.19$ (br s, $\left.1 \mathrm{H}, \mathrm{NH}\right), 4.70$ (br s, $\left.1 \mathrm{H}, \mathrm{OH}\right), 3.99$ (br s, $\left.1 \mathrm{H}, \mathrm{CHOH}\right), 3.52(1 \mathrm{H}, \mathrm{br}$ $\mathrm{s}, \mathrm{CHN}), 1.48\left(\mathrm{~s}, 9 \mathrm{H}, \mathrm{CCH}_{3}\right), 1.46\left(\mathrm{~s}, 9 \mathrm{H}, \mathrm{CCH}_{3}\right), 1.18\left(\mathrm{~d}, J=6.3 \mathrm{~Hz}, 3 \mathrm{H}, \mathrm{CH}_{3}\right), 1.03(\mathrm{~d}, J=6.9 \mathrm{~Hz}, 3 \mathrm{H}, \mathrm{CHCH}) ;{ }^{13} \mathrm{C} \mathrm{NMR}$ $\left(\mathrm{CDCl}_{3}, 75 \mathrm{MHz}, 52{ }^{\circ} \mathrm{C}\right) \delta 157.9,155.3,82.1,81.6,67.9,59.8,28.2,28.1,19.2,14.5 ; \mathrm{IR} v 3387(\mathrm{~m}), 3302(\mathrm{~m}), 2980(\mathrm{~s}), 2935$ (m), $2883(\mathrm{w}), 1714(\mathrm{~s}), 1516(\mathrm{~m}), 1480(\mathrm{~m}), 1456(\mathrm{~m}), 1395$ (s), $1368(\mathrm{~s}), 1333$ (s), 1288 (s), 1252 (s), 1159 (s), 1102 (s), 1051 (w), $1013(\mathrm{~m}), 923(\mathrm{~m}), 911(\mathrm{~m}), 851(\mathrm{w}), 827(\mathrm{w}), 784(\mathrm{w}), 759(\mathrm{w}), 734(\mathrm{~m}), 647(\mathrm{w}), 609(\mathrm{w}), 531$ (w); MS (ESI) 305.4 $(\mathrm{M}+\mathrm{H})$, $327.4(\mathrm{M}+\mathrm{Na})$; Anal. calcd for $\mathrm{C}_{14} \mathrm{H}_{28} \mathrm{~N}_{2} \mathrm{O}_{5}$ : C, 55.24; H, 9.27; N, 9.20. Found: C, 55.24; $\mathrm{H}, 9.41 ; \mathrm{N}, 8.96$.

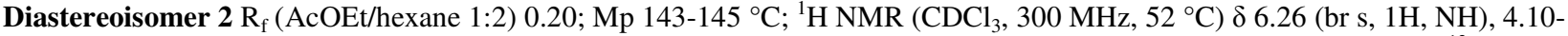
$3.97(\mathrm{~m}, 2 \mathrm{H}, \mathrm{CHN}$ and $\mathrm{CHOH}), 3.53($ br s, $1 \mathrm{H}, \mathrm{OH}), 1.47\left(\mathrm{~s}, 9 \mathrm{H}, \mathrm{CCH}_{3}\right), 1.46\left(\mathrm{~s}, 9 \mathrm{H}, \mathrm{CCH}_{3}\right), 1.12(\mathrm{~m}, 6 \mathrm{H}, \mathrm{CHCH}) ;{ }^{13} \mathrm{C} \mathrm{NMR}$ $\left(\mathrm{CDCl}_{3}, 75 \mathrm{MHz}, 52{ }^{\circ} \mathrm{C}\right) \delta 157.2,154.9,81.9,81.5,69.1,58.8,28.2,28.2,19.1,10.0 ; \mathrm{IR} v 3391$ (m, sh), 3309 (m), 2980 (s), 2935 (m), 1707 (s), 1480 (m), 1456 (m), 1394 (s), 1368 (s), 1292 (s), 1254 (s), 1160 (s), 1089 (s), 1005 (m), 978 (w), 919 (m), 909 (m), $858(w), 785(w), 760(w), 734(\mathrm{~m}), 647$ (w); HRMS (ESI) calcd for $\mathrm{C}_{14} \mathrm{H}_{28} \mathrm{~N}_{2} \mathrm{O}_{5} \mathrm{Na}^{+}(\mathrm{M}+\mathrm{Na})$ : 327.1890, found 327.1888; Anal. calcd for $\mathrm{C}_{14} \mathrm{H}_{28} \mathrm{~N}_{2} \mathrm{O}_{5}$ : C, 55.24; H, 9.27; N, 9.20. Found: C, 55.39; H, 9.22; N, 9.13.

$N$-(2-Benzyloxy-1-methyl-ethyl)- $N$ '-(tert-butoxycarbonyl)hydrazinecarboxylic acid tert-butyl ester (124)

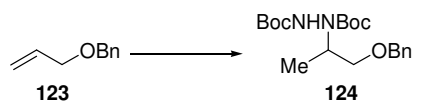

Following GP1 Hydrohydrazination product 124 (143 mg, $0.376 \mathrm{mmol}, 76 \%)$ was obtained as a colorless solid with benzylvinyl ether (123) $(77 \mu \mathrm{L}, 0.50 \mathrm{mmol}, 1.0$ equiv) in $5 \mathrm{~h}$ with 5 mol\% catalyst 20 after purification by column chromatography (AcOEt/hexane 1:10). $\mathrm{R}_{\mathrm{f}}$ (AcOEt/hexane 1:5) 0.27; $\mathrm{Mp} 99-100{ }^{\circ} \mathrm{C} ;{ }^{1} \mathrm{H} \mathrm{NMR}\left(\mathrm{CDCl}_{3}, 300 \mathrm{MHz}, 52{ }^{\circ} \mathrm{C}\right) \delta 7.36-7.23(\mathrm{~m}, 5 \mathrm{H}, \mathrm{Ar}$ $\mathrm{H}), 5.99$ (br s, $1 \mathrm{H}, \mathrm{NH}), 4.54\left(\mathrm{~d}, J=12.1 \mathrm{~Hz}, 1 \mathrm{H}, \mathrm{PhCH}_{2}\right), 4.48$ (br s, $\left.1 \mathrm{H}, \mathrm{CHN}\right), 4.45$ (d, $\left.J=12.1 \mathrm{~Hz}, 1 \mathrm{H}, \mathrm{PhCH}_{2}\right), 3.49(\mathrm{~m}, 1 \mathrm{H}$, $\left.\mathrm{CHNCH}_{2}\right), 3.38\left(\mathrm{~m}, 1 \mathrm{H}, \mathrm{CHNCH}_{2}\right), 1.46\left(\mathrm{~s}, 9 \mathrm{H}, \mathrm{CCH}_{3}\right), 1.46\left(\mathrm{~s}, 9 \mathrm{H}, \mathrm{CCH}_{3}\right), 1.13\left(\mathrm{~d}, 3 \mathrm{H}, J=6.9 \mathrm{~Hz}, \mathrm{CHNCH}_{3}\right) ;{ }^{13} \mathrm{C} \mathrm{NMR}$ $\left(\mathrm{CDCl}_{3}, 75 \mathrm{MHz}, 52{ }^{\circ} \mathrm{C}\right) \delta 155.9,155.0,138.4,128.3,127.6,80.9,80.8,72.8,71.3,52.0,28.3,28.2,14.4 ; \mathrm{IR} v 3307$ (m), 3065 (w), 3030 (w), 2979 (s), 2933 (m), 2869 (m), 1746 (s), 1707 (s), 1496 (m), 1479 (m), 1455 (m), 1392 (s), 1367 (s), 1337 (s), 1250 (s), 1155 (s), 1096 (s), 1064 (s), 1014 (m), 914 (w), 857 (w), 839 (w), 782 (w), 738 (m), 698 (m), 608 (w); MS (ESI) 403.1 $(\mathrm{M}+\mathrm{Na})$; Anal. calcd for $\mathrm{C}_{20} \mathrm{H}_{32} \mathrm{~N}_{2} \mathrm{O}_{5}: \mathrm{C}, 63.14 ; \mathrm{H}, 8.48 ; \mathrm{N}, 7.36$. Found: C, 62.85; H, 8.33; N, 7.49.

$N$-(2-Dimethoxy-1-methyl-ethyl)- $N$ '-(tert-butoxycarbonyl)hydrazinecarboxylic acid tert-butyl ester (126)

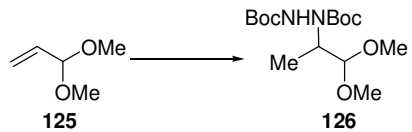

Following GP1 Hydrohydrazination product 126 (117 mg, 0.350 mmol, $70 \%$ (40\% pure, $30 \%$ co-fraction with N,N'-di-tertbutoxycarbonylhydrazine (4)) was obtained as a colorless viscous oil with acrolein-dimethylacetal (125) (freshly distilled, $59 \mu \mathrm{L}$, $0.50 \mathrm{mmol}, 1.0$ equiv), phenylsilane $(96 \mu \mathrm{L}, 0.77 \mathrm{mmol}, 1.5 \mathrm{equiv})$ and di-tert-butylazodicarboxylate $(\mathbf{2})(0.23 \mathrm{~g}, 1.0 \mathrm{mmol}, 2.0$ equiv) in $7 \mathrm{~h}$ with $5 \mathrm{~mol} \%$ catalyst $\mathbf{2 0}$ after purification by column chromatography (AcOEt/hexane 1:10). $\mathrm{R}_{\mathrm{f}}(\mathrm{AcOEt} / \mathrm{hexane} 1: 2)$ 0.36; ${ }^{1} \mathrm{H} \mathrm{NMR}\left(\mathrm{CDCl}_{3}, 300 \mathrm{MHz}, 52{ }^{\circ} \mathrm{C}\right) \delta 6.09$ (br s, $\left.1 \mathrm{H}, \mathrm{NH}\right), 4.26($ br s, $1 \mathrm{H}, \mathrm{CHN}), 4.26(\mathrm{~d}, J=5.3 \mathrm{~Hz}, 1 \mathrm{H}$, acetal H$), 3.35(\mathrm{~s}$, $\left.3 \mathrm{H}, \mathrm{OCH}_{3}\right), 3.31\left(\mathrm{~s}, 3 \mathrm{H}, \mathrm{OCH}_{3}\right), 1.46\left(\mathrm{~s}, 18 \mathrm{H}, \mathrm{CCH}_{3}\right), 1.14\left(\mathrm{~d}, J=6.5 \mathrm{~Hz}, 3 \mathrm{H}, \mathrm{CHNCH}_{3}\right) ;{ }^{13} \mathrm{C} \mathrm{NMR}\left(\mathrm{CDCl}_{3}, 75 \mathrm{MHz}, 52{ }^{\circ} \mathrm{C}\right) \delta$ 155.5, 154.7, 105.2, 81.0, 80.7, 54.8, 53.1, 28.3, 28.3, 12.9; IR v 3295 (m), $2979(\mathrm{~m}), 2936(\mathrm{~m}), 2836(\mathrm{w}), 1749(\mathrm{~s}), 1708(\mathrm{~s})$, $1479(\mathrm{~m}), 1456$ (m), 1394 (s), 1367 (s), $1319(\mathrm{~m}), 1241(\mathrm{~m}), 1155$ (s), $1102(\mathrm{~s}), 1051(\mathrm{~m}), 1012(\mathrm{w}), 973(\mathrm{w}), 952(\mathrm{w}), 859(\mathrm{w})$, $846(w), 782(w), 758(w)$; HRMS (ESI) calcd for $\mathrm{C}_{15} \mathrm{H}_{30} \mathrm{~N}_{2} \mathrm{O}_{6} \mathrm{Na}^{+}(\mathrm{M}+\mathrm{Na})$ : 357.1996, found 357.1993; Anal. calcd for $\mathrm{C}_{15} \mathrm{H}_{30} \mathrm{~N}_{2} \mathrm{O}_{6}: \mathrm{C}, 53.88 ; \mathrm{H}, 9.04 ; \mathrm{N}, 8.38$. Found: $\mathrm{C}, 53.60 ; \mathrm{H}, 8.98 ; \mathrm{N}, 8.21$.

$N$-(1-Methyl-4-oxo-pentyl)- $N$ '-(tert-butoxycarbonyl)hydrazinecarboxylic acid tert-butyl ester (128) 


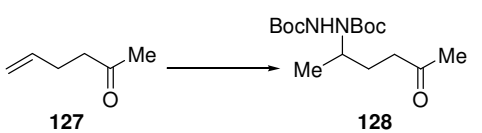

Following GP1 Hydrohydrazination product $128(125 \mathrm{mg}, 0.379 \mathrm{mmol}, 76 \%)$ was obtained as a colorless viscous oil with hexan-5-en-2-one (127) (freshly distilled, $56 \mu \mathrm{L}, 0.50 \mathrm{mmol}, 1.0$ equiv) in $3 \mathrm{~h}$ with $5 \mathrm{~mol} \%$ catalyst 20 after purification by column chromatography (AcOEt/hexane 1:5). $\mathrm{R}_{\mathrm{f}}(\mathrm{AcOEt} /$ hexane $1: 2) 0.37 ;{ }^{1} \mathrm{H} \mathrm{NMR}\left(\mathrm{CDCl}_{3}, 300 \mathrm{MHz}, 52{ }^{\circ} \mathrm{C}\right) \delta 6.11(\mathrm{br} \mathrm{s}, 1 \mathrm{H}$, $\mathrm{NH}$ ), 4.18 (br s, $1 \mathrm{H}, \mathrm{CHN}$ ), 2.51 (br s, 2H, $\mathrm{CH}_{2}$ ), 2.09 (s, 3H, $\mathrm{COCH}_{3}$ ), 1.79 (br s, 1H, $\mathrm{CH}_{2}$ ), 1.52 (br s, $\left.1 \mathrm{H}, \mathrm{CH}_{2}\right), 1.45(\mathrm{~s}, 9 \mathrm{H}$,

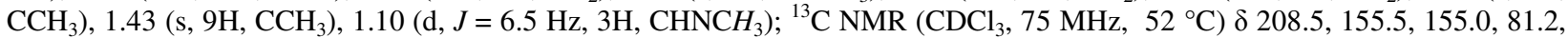
80.8, 52.9, 40.8, 30.0, 28.3, 28.2, 27.8, 18.2; IR v $3318(\mathrm{~m}), 2979(\mathrm{~s}), 2934(\mathrm{~m}), 1746$ (s), $1710(\mathrm{~s}), 1480(\mathrm{~m}), 1456(\mathrm{~m}), 1394(\mathrm{~s})$, 1367 (s), 1338 (s), 1246 (s), 1160 (s), 1117 (s), $1082(\mathrm{~m}), 1047$ (m), $1014(\mathrm{~m}), 917$ (w), $858(\mathrm{w}), 782(\mathrm{w}), 760(\mathrm{~m}), 736(\mathrm{w}), 689$ (w), $647(w), 598(w)$; HRMS (ESI) calcd for $\mathrm{C}_{16} \mathrm{H}_{30} \mathrm{~N}_{2} \mathrm{O}_{5} \mathrm{Na}^{+}(\mathrm{M}+\mathrm{Na})$ : 353.2047, found 353.2060; Anal. calcd for $\mathrm{C}_{16} \mathrm{H}_{30} \mathrm{~N}_{2} \mathrm{O}_{5}$ : C, 58.16; H, 9.10; N, 8.48. Found: C, 58.36; H, 9.10; N, 8.29.

\section{$N$-(3-Bromo-1-methyl-propyl)- $N$ '-(tert-butoxycarbonyl)hydrazinecarboxylic acid tert-butyl ester (130)

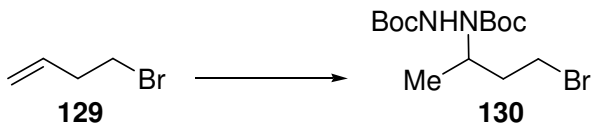

Following GP1 Hydrohydrazination product 130 (166 mg, $0.452 \mathrm{mmol}, 90 \%)$ was obtained as a colorless solid with 4bromobutene (129) $(51 \mu \mathrm{L}, 0.50 \mathrm{mmol}, 1.0$ equiv) in $5 \mathrm{~h}$ with $5 \mathrm{~mol} \%$ catalyst 20 after purification by column chromatography (AcOEt/hexane 1:15). $\mathrm{R}_{\mathrm{f}}$ (AcOEt/hexane 1:5) 0.33; $\mathrm{Mp} \mathrm{88-90}{ }^{\circ} \mathrm{C} ;{ }^{1} \mathrm{H} \mathrm{NMR}\left(\mathrm{CDCl}_{3}, 300 \mathrm{MHz}, 52{ }^{\circ} \mathrm{C}\right) \delta 6.06(\mathrm{br}, 1 \mathrm{H}, \mathrm{NH}), 4.38$ $(\mathrm{m}, 1 \mathrm{H}, \mathrm{CHN}), 3.45\left(\mathrm{~m}, 2 \mathrm{H}, \mathrm{CH}_{2} \mathrm{Br}\right), 2.13\left(\mathrm{~m}, 1 \mathrm{H}, \mathrm{CH}_{2} \mathrm{CHN}\right), 1.82\left(\mathrm{~m}, 1 \mathrm{H}, \mathrm{CH}_{2} \mathrm{CHN}\right), 1.46\left(\mathrm{~s}, 18 \mathrm{H}, \mathrm{CCH}_{3}\right), 1.12(\mathrm{~d}, J=6.5 \mathrm{~Hz}$, $\left.3 \mathrm{H}, \mathrm{CHNCH}_{3}\right) ;{ }^{13} \mathrm{C} \mathrm{NMR}\left(\mathrm{CDCl}_{3}, 75 \mathrm{MHz}, 52{ }^{\circ} \mathrm{C}\right) \delta 156.0,154.7,81.3,52.2,37.5,30.5,28.3,28.2,18.0 ; \mathrm{IR} v 3315(\mathrm{~m}), 2979$ (s), $2934(\mathrm{~m}), 1707(\mathrm{~s}), 1479(\mathrm{~m}), 1456(\mathrm{~m}), 1394(\mathrm{~s}), 1368(\mathrm{~s}), 1336(\mathrm{~m}), 1305(\mathrm{~m}), 1243$ (s), 1160 (s), $1113(\mathrm{~m}), 1085$ (w), 1054 (m), 1018 (w), 1008 (w), $912(w), 856$ (w), $784(w), 760$ (m), 649 (w), 558 (w); HRMS (ESI) calcd for $\mathrm{C}_{14} \mathrm{H}_{27} \mathrm{~N}_{2} \mathrm{O}_{4} \mathrm{BrNa}^{+}$ $(\mathrm{M}+\mathrm{Na})$ : 389.1052, 391.1031, found 389.1050, 391.1031; Anal. calcd for $\mathrm{C}_{14} \mathrm{H}_{27} \mathrm{~N}_{2} \mathrm{O}_{4} \mathrm{Br}$ : C, 45.78; H, 7.41; N, 7.63. Found: C, 45.98; H, 7.48; N, 7.63.

$N$-(1-Furan-2-yl-ethyl)- $N$ '-(tert-butoxycarbonyl)hydrazinecarboxylic acid tert-butyl ester (131)

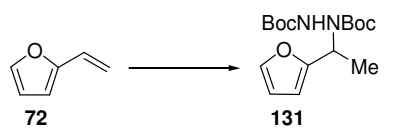

Following GP2 Hydrohydrazination product $131(110 \mathrm{mg}, 0.337 \mathrm{mmol}, 68 \%)$ was obtained as a colorless solid with vinylfuran $72(55 \%$ pure, $86 \mathrm{mg}, 0.50 \mathrm{mmol}, 1.0$ equiv) in $12 \mathrm{~h}$ with $2.5 \mathrm{~mol} \%$ catalyst 20 after purification by column chromatography (AcOEt/hexane 1:10). Following GP4 Hydrohydrazination product 131 (103 mg, $0.316 \mathrm{mmol}, 63 \%)$ was obtained as a colorless solid with vinylfuran 72 (55\% pure, $86 \mathrm{mg}, 0.50 \mathrm{mmol}, 1.0$ equiv) in $12 \mathrm{~h}$ with $1 \mathrm{~mol} \%$ catalyst 23 after purification by column chromatography (AcOEt/hexane 1:10). $\mathrm{R}_{\mathrm{f}}(\mathrm{AcOEt} / \mathrm{hexane} 1: 5)$ 0.40; $\mathrm{Mp} 110-111{ }^{\circ} \mathrm{C} ;{ }^{1} \mathrm{H} \mathrm{NMR}\left(\mathrm{CDCl}_{3}, 300 \mathrm{MHz}, 52{ }^{\circ} \mathrm{C}\right) \delta 7.31$ $(\mathrm{m}, 1 \mathrm{H}$, furan $\mathrm{H}), 6.27(\mathrm{~m}, 1 \mathrm{H}$, furan $\mathrm{H}), 6.17(\mathrm{~m}, 1 \mathrm{H}$, furan $\mathrm{H}), 5.96($ br s, $1 \mathrm{H}, \mathrm{NH}), 5.42(\mathrm{br} \mathrm{m}, 1 \mathrm{H}, \mathrm{CHN}), 1.50(\mathrm{~m}, 3 \mathrm{H}$,

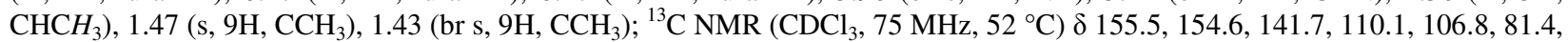
80.8, 50.7, 28.2, 28.1, 15.7; IR v $3317(\mathrm{~m}), 3153(\mathrm{w}), 2980(\mathrm{~m}), 2935$ (m), 1705 (s), $1504(\mathrm{~m}), 1479(\mathrm{~m}), 1456(\mathrm{~m}), 1393(\mathrm{~s}), 1368$ (s), $1311(\mathrm{~s}), 1242$ (s), 1161 (s), $1084(\mathrm{w}), 1053(\mathrm{~m}), 1010(\mathrm{~m}), 995$ (m), 922 (w), 909 (w), 885 (w), 858 (w), 845 (w), 810 (w), $754(\mathrm{~m}), 691(\mathrm{w}), 648(\mathrm{w}), 600(\mathrm{w}), 485(\mathrm{w}), 460(\mathrm{w})$; MS (ESI) $327.1(\mathrm{M}+\mathrm{H}), 349.1(\mathrm{M}+\mathrm{Na})$; Anal. calcd for $\mathrm{C}_{16} \mathrm{H}_{26} \mathrm{~N}_{2} \mathrm{O}_{5}$ : C, 58.88; H, 8.03; N, 8.58. Found: C, 59.05; H, 8.10; N, 8.56.

$N$-(1-Furan-3-yl-ethyl)- $N$ '-(tert-butoxycarbonyl)hydrazinecarboxylic acid tert-butyl ester (132)

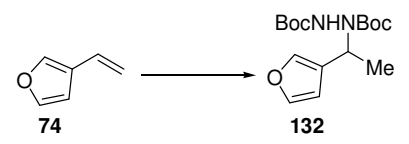

Following GP2 Hydrohydrazination product 132 (130 mg, $0.398 \mathrm{mmol}, 75 \%)$ was obtained as a colorless solid with vinylfuran 74 (41\% pure, $121 \mathrm{mg}, 0.527 \mathrm{mmol}, 1.00$ equiv) in $18 \mathrm{~h}$ with $2.5 \mathrm{~mol} \%$ catalyst 20 after purification by column chromatography (AcOEt/hexane 1:9). Following GP3 Hydrohydrazination product 132 (115 mg, $0.352 \mathrm{mmol}, 72 \%)$ was obtained as a colorless solid with vinylfuran 74 (41\% pure, $112 \mathrm{mg}, 0.488 \mathrm{mmol}, 1.00$ equiv) in $18 \mathrm{~h}$ with $1 \mathrm{~mol} \%$ catalyst 23 after purification by column chromatography (AcOEt/hexane 1:10). $\mathrm{R}_{\mathrm{f}}\left(\mathrm{AcOEt} /\right.$ hexane 1:5) 0.40; Mp 79-81 ${ }^{\circ} \mathrm{C} ;{ }^{1} \mathrm{H} \mathrm{NMR}\left(\mathrm{CDCl}{ }_{3}, 300 \mathrm{MHz}, 52{ }^{\circ} \mathrm{C}\right) \delta$ $7.35(\mathrm{~m}, 2 \mathrm{H}$, furan $\mathrm{H}), 6.35$ (s, $1 \mathrm{H}$, furan $\mathrm{H}), 5.87$ (br s, $1 \mathrm{H}, \mathrm{NH}), 5.35-5.29$ (br m, $1 \mathrm{H}, \mathrm{CHN}), 1.49\left(\mathrm{~s}, 9 \mathrm{H}, \mathrm{CCH}_{3}\right), 1.47$ (m, 3H, $\left.\mathrm{CHCH}_{3}\right), 1.45$ (br s, 9H, $\left.\mathrm{CCH}_{3}\right) ;{ }^{13} \mathrm{C} \mathrm{NMR}\left(\mathrm{CDCl}_{3}, 75 \mathrm{MHz}, 52{ }^{\circ} \mathrm{C}\right) \delta 155.5,154.5,142.8,139.7,125.8,109.7,81.2,80.8,48.9$, 28.3, 28.2, 17.2; IR v $3314(\mathrm{~m}), 3150(\mathrm{w}), 2980(\mathrm{~m}), 2935$ (m), $1704(\mathrm{~s}), 1504(\mathrm{~m}), 1479(\mathrm{~m}), 1456(\mathrm{~m}), 1393(\mathrm{~s}), 1368(\mathrm{~s}), 1316$ (m), $1251(\mathrm{~m}), 1163(\mathrm{~s}), 1098(\mathrm{~m}), 1058(\mathrm{~m}), 1019(\mathrm{~m}), 998(\mathrm{~m}), 952(\mathrm{w}), 910(\mathrm{w}), 875(\mathrm{~m}), 858(\mathrm{w}), 844(\mathrm{w}), 798(\mathrm{w}), 756(\mathrm{w})$, $733(\mathrm{w}), 684(\mathrm{w}), 648$ (w), 601 (m), 485 (w), 459 (w), 440 (w), 420 (w); MS (ESI) 327.1 (M+H), 349.1 (M+Na), 675.0 (2M+Na); Anal. calcd for $\mathrm{C}_{16} \mathrm{H}_{26} \mathrm{~N}_{2} \mathrm{O}_{5}$ : C, 58.88; H, 8.03; N, 8.58. Found: C, 58.97; H, 8.12; N, 8.60.

$N$-(1-Thiophene-2-yl-ethyl)- $N$ '-(tert-butoxycarbonyl)hydrazinecarboxylic acid tert-butyl ester (133) 


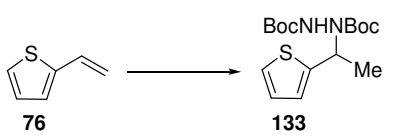

Following GP2 Hydrohydrazination product $133(144 \mathrm{mg}, 0.420 \mathrm{mmol}, 84 \%)$ was obtained as a colorless solid with vinylthiophene 76 (90\% pure, $62 \mathrm{mg}, 0.50 \mathrm{mmol}, 1.0$ equiv) in $18 \mathrm{~h}$ with $2.5 \mathrm{~mol} \%$ catalyst 20 after purification by column chromatography (AcOEt/hexane 1:10). Following GP4 Hydrohydrazination product 133 (146 mg, 0.426 mmol, 85\%) was obtained as a colorless solid with vinylthiophene $\mathbf{7 6}$ (90\% pure, $62 \mathrm{mg}, 0.50 \mathrm{mmol}, 1.0$ equiv) in $18 \mathrm{~h}$ with $1 \mathrm{~mol} \%$ catalyst $\mathbf{2 3}$ after purification by column chromatography (AcOEt/hexane 1:10). $\mathrm{R}_{\mathrm{f}}\left(\mathrm{AcOEt} /\right.$ hexane 1:5) 0.40; $\mathrm{Mp} \mathrm{73-75}{ }^{\circ} \mathrm{C} ;{ }^{1} \mathrm{H} \mathrm{NMR}\left(\mathrm{CDCl}{ }_{3}\right.$, $\left.300 \mathrm{MHz}, 52{ }^{\circ} \mathrm{C}\right) \delta 7.19-7.16(\mathrm{~m}, 1 \mathrm{H}$, furan $\mathrm{H}), 6.94-6.91(\mathrm{~m}, 2 \mathrm{H}$, furan $\mathrm{H}), 5.96($ br s, $1 \mathrm{H}, \mathrm{NH}), 5.65($ br m, $1 \mathrm{H}, \mathrm{CHN}), 1.57(\mathrm{~d}, J$

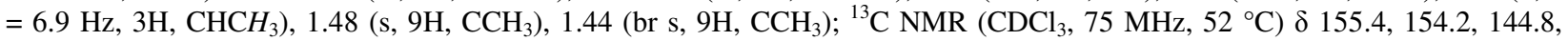
126.4, 124.6, 124.3, 81.5, 80.9, 51.9, 28.3, 28.2, 18.4; IR v $3316(\mathrm{~m}), 3106(\mathrm{w}), 2979(\mathrm{~m}), 2934(\mathrm{~m}), 1704(\mathrm{~s}), 1479(\mathrm{~m}), 1456$ (m), 1392 (s), 1368 (s), 1315 (s), 1239 (s), 1164 (s), 1087 (w), 1058 (m), 1030 (w), 1015 (m), 984 (w), 910 (w), 854 (m), 842 (w), 756 (m), 699 (m), $582(\mathrm{w}), 475(\mathrm{w})$; MS (ESI) $343.0(\mathrm{M}+\mathrm{H}), 365.1(\mathrm{M}+\mathrm{Na})$; Anal. calcd for $\mathrm{C}_{16} \mathrm{H}_{26} \mathrm{~N}_{2} \mathrm{O}_{4} \mathrm{~S}: \mathrm{C}, 56.12 ; \mathrm{H}, 7.65 ; \mathrm{N}$, 8.18. Found: C, 56.35; H, 7.70; N, 8.22.

$N$-(1-(1-Methyl-1H-imidazole-2-yl)-ethyl)- $N$ '-(tert-butoxycarbonyl)hydrazinecarboxylic acid tert-butyl ester (134)

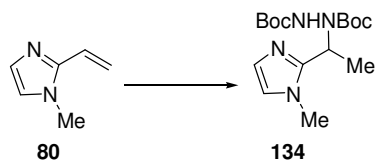

Following GP2 Hydrohydrazination product $134(102 \mathrm{mg}, 0.300 \mathrm{mmol}, 60 \%)$ was obtained as a colorless solid with vinylimidazole 80 (54 mg, $0.50 \mathrm{mmol}, 1.0$ equiv) in $8 \mathrm{~h}$ with $2.5 \mathrm{~mol} \%$ catalyst 20 after purification by column chromatography $\left(\mathrm{CH}_{2} \mathrm{Cl}_{2} / \mathrm{MeOH} 14: 1\right)$. Following GP4 Hydrohydrazination product 134 (142 mg, $\left.0.417 \mathrm{mmol}, 83 \%\right)$ was obtained as a colorless solid with vinylimidazole 80 (54 mg, $0.50 \mathrm{mmol}, 1.0$ equiv) in $5 \mathrm{~h}$ with 1 mol\% catalyst 23 after purification by column chromatography $\left(\mathrm{CH}_{2} \mathrm{Cl}_{2} / \mathrm{MeOH} 14: 1\right)$. Mp 131-135 ${ }^{\circ} \mathrm{C} ;{ }^{1} \mathrm{H}$ NMR $\left(\mathrm{CDCl}_{3}, 300 \mathrm{MHz}, 52{ }^{\circ} \mathrm{C}\right) \delta 6.90(\mathrm{~d}, J=0.8 \mathrm{~Hz}, 1 \mathrm{H}$, imidazole $\mathrm{H}), 6.77$ (d, $J=1.1 \mathrm{~Hz}, 1 \mathrm{H}$, imidazole H), 6.40 (br s, 1H, NH), 5.54 (br m, 1H, CHN), 3.67 (s, 3H, NCH 3 ), 1.57 (d, $J=7.0 \mathrm{~Hz}$,

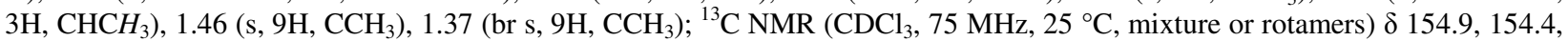
147.7, 147.0, 145.7, 144.8, 126.8, 120.9, 81.2, 80.3, 47.5, 46.9, 32.7, 28.1, 16.1; IR v $3314(\mathrm{w}), 3166$ (w), $2980(\mathrm{~m}), 2934$ (w), 1731 (s), 1697 (s), 1642 (w), 1547 (w), 1496 (m), 1479 (m), 1454 (m), 1393 (s), 1367 (s), 1319 (s), 1282 (s), 1245 (s), 1170 (s), 1125 (w), 1094 (w), 1057 (m), 1023 (w), 994 (w), 922 (w), 858 (w), $756(w), 733(\mathrm{~m}), 646(\mathrm{w}), 590(\mathrm{w}), 521$ (w); MS (ESI) 341.4 $(\mathrm{M}+\mathrm{H}), 363.2(\mathrm{M}+\mathrm{Na})$; Anal. calcd for $\mathrm{C}_{16} \mathrm{H}_{28} \mathrm{~N}_{4} \mathrm{O}_{4}$ : C, 56.45; H, 8.29; N, 16.46. Found: C, 56.54; H, 8.43; N, 16.17.

$N$-(1-Pyridin-2-yl-ethyl)- $N$ '-(tert-butoxycarbonyl)hydrazinecarboxylic acid tert-butyl ester (136)

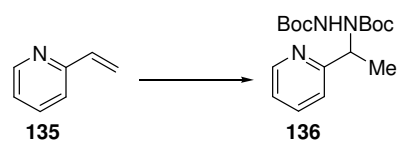

Following GP2 Hydrohydrazination product $136(130 \mathrm{mg}, 0.385 \mathrm{mmol}$, 77\%) was obtained as a colorless gel with vinylpyridine 135 (filered over $\mathrm{Al}_{2} \mathrm{O}_{3}, 54 \mu \mathrm{L}, 0.50 \mathrm{mmol}, 1.0$ equiv) in $4 \mathrm{~h}$ with $2.5 \mathrm{~mol} \%$ catalyst 20 after purification by column chromatography (AcOEt/hexane 1:5-1:2). Following GP4 Hydrohydrazination product 136 (132 mg, $0.391 \mathrm{mmol}, 78 \%)$ was obtained as a colorless gel with vinylpyridine 135 (filered over $\mathrm{Al}_{2} \mathrm{O}_{3}, 54 \mu \mathrm{L}, 0.50$ mmol, 1.0 equiv) in $13 \mathrm{~h}$ with $1 \mathrm{~mol} \%$ catalyst 23 after purification by column chromatography (AcOEt/hexane 1:3). $\mathrm{R}_{\mathrm{f}}(\mathrm{AcOEt} / \mathrm{hexane} 1: 2) 0.30 ;{ }^{1} \mathrm{H} \mathrm{NMR}\left(\mathrm{CDCl}{ }_{3}, 300 \mathrm{MHz}\right.$, $\left.52{ }^{\circ} \mathrm{C}\right) \delta 8.47(\mathrm{~d}, J=4.7 \mathrm{~Hz}, 1 \mathrm{H}$, pyridine $\mathrm{H}), 7.59(\mathrm{dd}, J=9.4,7.7 \mathrm{~Hz}, 1 \mathrm{H}$, pyridine $\mathrm{H}), 7.22(\mathrm{br} \mathrm{m}, 1 \mathrm{H}$, pyridine $\mathrm{H}), 7.10(\mathrm{dd}, J$ = 7.2, $5.2 \mathrm{~Hz}, 1 \mathrm{H}$, pyridine $\mathrm{H}), 6.80(\mathrm{br} \mathrm{s}, 1 \mathrm{H}, \mathrm{NH}), 5.37$ (br m, $1 \mathrm{H}, \mathrm{CHN}), 1.53\left(\mathrm{~d}, J=7.1 \mathrm{~Hz}, 3 \mathrm{H}, \mathrm{CHCH}_{3}\right), 1.44\left(\mathrm{~s}, 9 \mathrm{H}, \mathrm{CCH}_{3}\right)$, 1.38 (br s, 9H, $\left.\mathrm{CCH}_{3}\right) ;{ }^{13} \mathrm{C} \mathrm{NMR}\left(\mathrm{CDCl}_{3}, 75 \mathrm{MHz}, 52{ }^{\circ} \mathrm{C}\right) \delta 161.6,155.4,148.7,136.3,134.0,121.8,121.2,81.1,80.4,57.2$, 28.3, 28.2, 17.5; IR v 3312 (w), 2980 (m), 2934 (w), 1708 (s), 1594 (w), $1572(w), 1477$ (m), 1456 (m), 1435 (w), 1392 (s), 1367 (s), $1317(\mathrm{~m}), 1245$ (s), $1161(\mathrm{~s}), 1092(\mathrm{w}), 1049$ (w), $1021(\mathrm{w}), 989(\mathrm{w}), 913(\mathrm{w}), 858(\mathrm{w}), 779(\mathrm{w}), 751(\mathrm{w}), 734(\mathrm{w}), 700(\mathrm{w})$, $668(w), 592(w), 501(w)$; MS (ESI) $338.1(\mathrm{M}+\mathrm{H}), 360.0(\mathrm{M}+\mathrm{Na})$; Anal. calcd for $\mathrm{C}_{17} \mathrm{H}_{27} \mathrm{~N}_{3} \mathrm{O}_{4}$ : C, 60.51; H, 8.07; N, 12.45. Found: C, 60.36; H, 8.11; N, 12.26.

$N$-(1-Pyrazin-2-yl-ethyl)-N'-(tert-butoxycarbonyl)hydrazinecarboxylic acid tert-butyl ester (137)

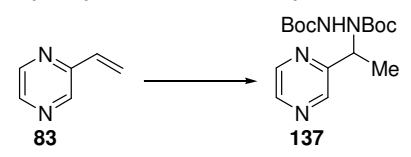

Following GP2 Hydrohydrazination product $137(110 \mathrm{mg}, 0.325 \mathrm{mmol}$, 63\%) was obtained as a colorless gel with vinylpyrazine 83 (55 mg, $0.52 \mathrm{mmol}, 1.0$ equiv) in $24 \mathrm{~h}$ with $2.5 \mathrm{~mol} \%$ catalyst 20 after purification by column chromatography (AcOEt/hexane 1:2). Following GP4 Hydrohydrazination product 137 (112 $\mathrm{mg}, 0.331 \mathrm{mmol}, 64 \%)$ was obtained as a colorless gel with vinylpyrazine $83(55 \mathrm{mg}, 0.52 \mathrm{mmol}, 1.0$ equiv) in $10 \mathrm{~h}$ with $1 \mathrm{~mol} \%$ catalyst 23 after purification by column chromatography (AcOEt/hexane 1:4-1:2). $\mathrm{R}_{\mathrm{f}}$ (AcOEt/hexane 1:2) $0.20 ;{ }^{1} \mathrm{H} \mathrm{NMR}\left(\mathrm{CDCl}_{3}, 300 \mathrm{MHz}, 52{ }^{\circ} \mathrm{C}\right) \delta 8.58(\mathrm{~s}, 1 \mathrm{H}$, pyrazine $\mathrm{H}), 8.41(\mathrm{~m}, 2 \mathrm{H}$, pyrazine $\mathrm{H}), 6.52(\mathrm{br} \mathrm{s}, 1 \mathrm{H}, \mathrm{NH}), 5.45$ (br m, $1 \mathrm{H}, \mathrm{CHN}), 1.56(\mathrm{~d}, \mathrm{~J}=7.2 \mathrm{~Hz}, 3 \mathrm{H}, \mathrm{CHCH}), 1.42(\mathrm{~s}, 9 \mathrm{H}$, 
$\mathrm{CCH}_{3}$ ), 1.39 (br s, 9H, $\left.\mathrm{CCH}_{3}\right) ;{ }^{13} \mathrm{C} \mathrm{NMR}\left(\mathrm{CDCl}_{3}, 75 \mathrm{MHz}, 52{ }^{\circ} \mathrm{C}\right) \delta 156.8,155.3,154.9,143.6,143.3,142.9,81.5,80.8,55.6$, 28.2, 16.8; IR v $3312(\mathrm{w}), 2980$ (m), 2935 (w), 1706 (s), 1477 (m), 1456 (m), 1392 (s), 1368 (s), 1317 (s), 1247 (s), 1159 (s), 1095 (m), 1053 (m), 1019 (m), 994 (w), 913 (w), 852 (m), 762 (w), 734 (m), 648 (w), 595 (w), 461 (w); MS (ESI) 361.2 (M+Na); Anal. calcd for $\mathrm{C}_{16} \mathrm{H}_{26} \mathrm{~N}_{4} \mathrm{O}_{4}$ : C, 56.79; $\mathrm{H}, 7.74 ; \mathrm{N}, 16.56$. Found: $\mathrm{C}, 56.99 ; \mathrm{H}, 7.85 ; \mathrm{N}, 16.35$.

\section{$N$-(1-(1H-Indol-2-yl)-ethyl)- $N$ '-(tert-butoxycarbonyl)hydrazinecarboxylic acid tert-butyl ester (138)}

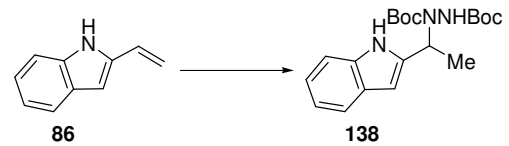

Following GP2 Hydrohydrazination product $138(153 \mathrm{mg}, 0.408 \mathrm{mmol}, 82 \%)$ was obtained as a colorless solid with vinylindole 86 (73 mg, $0.50 \mathrm{mmol}, 1.0$ equiv) in $5 \mathrm{~h}$ with $2.5 \mathrm{~mol} \%$ catalyst 20 after purification by column chromatography (AcOEt/hexane 1:7). Following GP4 Hydrohydrazination product $138(135 \mathrm{mg}, 0.360 \mathrm{mmol}, 72 \%)$ was obtained as a colorless solid with vinylindole 86 (73 mg, $0.50 \mathrm{mmol}, 1.0$ equiv) in $5 \mathrm{~h}$ with 1 mol\% catalyst 23 after purification by column chromatography (AcOEt/hexane 1:7). $\mathrm{R}_{\mathrm{f}}(\mathrm{AcOEt} / \mathrm{hexane} 1: 5)$ 0.35; Mp 73-75 ${ }^{\circ} \mathrm{C} ;{ }^{1} \mathrm{H} \mathrm{NMR}\left(\mathrm{CDCl}_{3}, 300 \mathrm{MHz}, 52{ }^{\circ} \mathrm{C}\right) \delta 9.46(\mathrm{br} \mathrm{s}, 1 \mathrm{H}$, indole NH), $7.55(\mathrm{~d}, J=7.8 \mathrm{~Hz}, 1 \mathrm{H}$, indole $\mathrm{CH}), 7.34(\mathrm{~d}, J=8.1 \mathrm{~Hz}, 1 \mathrm{H}$, indole $\mathrm{CH}), 7.17-7.04(\mathrm{~m}, 2 \mathrm{H}$, indole $\mathrm{CH}), 6.35(\mathrm{~s}, 1 \mathrm{H}$, indole $\mathrm{CH})$, 6.24 (br s, 1H, hydrazine NH), 5.47 (br m, $1 \mathrm{H}, \mathrm{CHN}), 1.64$ (d, J = 7.2 Hz, 3H, $\left.\mathrm{CHCH}_{3}\right), 1.46\left(\mathrm{~s}, 18 \mathrm{H}, \mathrm{CCH}_{3}\right) ;{ }^{13} \mathrm{C} \mathrm{NMR}\left(\mathrm{CDCl}_{3}\right.$, $\left.75 \mathrm{MHz}, 52{ }^{\circ} \mathrm{C}\right) \delta 156.1,154.9,139.3,136.1,133.9,128.1,121.5,120.0,119.3,110.9,99.5,81.9,81.5,52.6,28.2,28.1,16.7$; IR v $3320(\mathrm{~m}), 3059$ (w), 2980 (m), 2935 (w), 1694 (s), 1619 (w), 1585 (w), 1479 (m), 1456 (m), 1393 (s), 1368 (s), 1302 (m), 1281 (m), 1252 (m), 1157 (s), 1092 (w), 1061 (w), 1025 (w), 1000 (w), 959 (w), 910 (m), 855 (w), 789 (w), 735 (m), 700 (w), 649 (w), $555(w)$; MS (ESI) $376.3(\mathrm{M}+\mathrm{H}), 398.2(\mathrm{M}+\mathrm{Na})$; Anal. calcd for $\mathrm{C}_{20} \mathrm{H}_{29} \mathrm{~N}_{3} \mathrm{O}_{4}: \mathrm{C}, 63.98 ; \mathrm{H}, 7.78 ; \mathrm{N}, 11.19$. Found: C, 64.18; $\mathrm{H}$, $7.92 ; \mathrm{N}, 10.95$.

$N$-[1-(4-Amino-phenyl)-ethyl]- $N$ '-(tert-butoxycarbonyl)hydrazinecarboxylic acid tert-butyl ester (139)

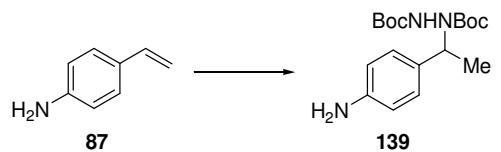

Following GP2 Hydrohydrazination product 139 was obtained as the minor product $(<40 \%)$ of a mixture of non-identified products with 4-vinylaniline 87 (90\%, $67 \mathrm{mg}, 0.50 \mathrm{mmol}, 1.0$ equiv) in $20 \mathrm{~h}$ with $2.5 \mathrm{~mol} \%$ catalyst 20. Purification by column chromatography (AcOEt/hexane 1:1) was not successful.

Following GP4 Hydrohydrazination product 139 (144 mg, 81\% pure, $0.332 \mathrm{mmol}, 66 \%$ ) was obtained as a colorless solid with 4-vinylaniline $87(90 \%, 67 \mathrm{mg}, 0.50 \mathrm{mmol}, 1.0$ equiv) in $4 \mathrm{~h}$ with 1 mol\% catalyst 23 after purification by column chromatography (AcOEt/hexane 1:1).

Via Deprotection of 140

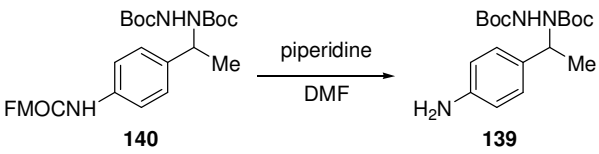

A mixture of piperidine $(0.16 \mathrm{~mL}, 1.6 \mathrm{mmol}, 9.2$ equiv) and DMF $(2.8 \mathrm{~mL})$ was added to hydrohydrazination product 140 (100 $\mathrm{mg}, 0.174 \mathrm{mmol}, 1.00$ equiv) and the reaction mixture was stirred $14 \mathrm{~h}$ at $23{ }^{\circ} \mathrm{C}$. The solvent was removed under reduced pressure and the isolated product was purified by column chromatography (AcOEt/hexane 1:1) to afford $\mathbf{1 3 9}(55 \mathrm{mg}, 0.16 \mathrm{mmol}, 90 \%)$ as a colorless solid. $\mathrm{R}_{\mathrm{f}}$ (AcOEt/hexane 1:1) 0.35; Mp 62-67 ${ }^{\circ} \mathrm{C} ;{ }^{1} \mathrm{H} \mathrm{NMR}\left(\mathrm{CDCl}_{3}, 300 \mathrm{MHz}, 52{ }^{\circ} \mathrm{C}\right) \delta 7.12(\mathrm{~d}, \mathrm{~J}=8.2 \mathrm{~Hz}, 2 \mathrm{H}, \mathrm{Ar} \mathrm{H})$, $6.62(\mathrm{~d}, J=8.5 \mathrm{~Hz}, 2 \mathrm{H}, \mathrm{Ar} \mathrm{H}), 5.83$ (br s, $1 \mathrm{H}$, hydrazine $\mathrm{NH}), 5.35$ (br m, 1H, CHN), 3.61 (br s, 2H, NH$), 1.49-1.47(\mathrm{~m}, 3 \mathrm{H}$, $\left.\mathrm{CHCH}_{3}\right), 1.47\left(\mathrm{~s}, 9 \mathrm{H}, \mathrm{CCH}_{3}\right), 1.44\left(\right.$ br s, $\left.9 \mathrm{H}, \mathrm{CCH}_{3}\right) ;{ }^{13} \mathrm{C} \mathrm{NMR}\left(\mathrm{CDCl}_{3}, 75 \mathrm{MHz}, 52{ }^{\circ} \mathrm{C}\right) \delta 155.7,154.8,145.8,131.1,128.4$, 114.9, 81.0, 80.7, 55.3, 28.3, 28.1, 16.9; IR $\vee 3366(\mathrm{w}), 2979(\mathrm{~m}), 2933(\mathrm{w}), 1694(\mathrm{~s}), 1626(\mathrm{~m}), 1548(\mathrm{w}), 1518(\mathrm{~m}), 1479(\mathrm{~m})$, 1454 (m), 1392 (s), 1367 (s), 1317 (m), 1253 (m), 1172 (s), 1048 (w), 1022 (w), 992 (w), $911(\mathrm{w}), 832$ (w), 756 (w), 734 (m), 647 $(\mathrm{w}), 620(\mathrm{w}), 535(\mathrm{w})$; MS (ESI) $352.2(\mathrm{M}+\mathrm{H}), 374.2(\mathrm{M}+\mathrm{Na}), 703.0(2 \mathrm{M}+\mathrm{H}), 725.0(2 \mathrm{M}+\mathrm{Na})$; Anal. calcd for $\mathrm{C}_{18} \mathrm{H}_{29} \mathrm{~N}_{3} \mathrm{O}_{4}$ : $\mathrm{C}$, $61.52 ; \mathrm{H}, 8.32 ; \mathrm{N}, 11.96$. Found: $\mathrm{C}, 61.81 ; \mathrm{H}, 8.58 ; \mathrm{N}, 11.72$.

$N$-\{1-[4-(9,9a-Dihydro-4aH-fluoren-9-ylmethoxycarbonylamino)-phenyl]-ethyl\}- $N$ '-(tertbutoxycarbonyl)hydrazinecarboxylic acid tert-butyl ester (140)

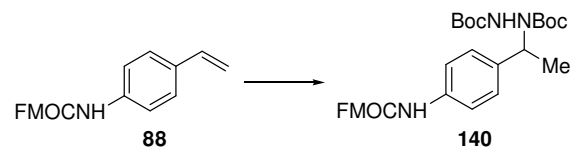

Following GP2 Hydrohydrazination product 140 (282 mg, $0.492 \mathrm{mmol}, 98 \%)$ was obtained as a colorless solid with protected vinylaniline 88 (171 mg, $0.501 \mathrm{mmol}, 1.00$ equiv) in 4 h with $2.5 \mathrm{~mol} \%$ catalyst 20 after purification by column chromatography (AcOEt/ $\mathrm{CH}_{2} \mathrm{Cl}_{2}$ 1:10). Following GP4 Hydrohydrazination product 140 (202 $\left.\mathrm{mg}, 0.352 \mathrm{mmol}, 70 \%\right)$ was obtained as a colorless solid with protected vinylaniline 88 (171 mg, $0.501 \mathrm{mmol}, 1.00$ equiv) in $4 \mathrm{~h}$ with $1 \mathrm{~mol} \%$ catalyst 23 after purification by column chromatography (AcOEt/CH $\mathrm{CH}_{2}$ 1:10). $\mathrm{Mp} \mathrm{105-112}{ }^{\circ} \mathrm{C} ;{ }^{1} \mathrm{H} \mathrm{NMR}\left(\mathrm{CDCl}_{3}, 300 \mathrm{MHz}, 52{ }^{\circ} \mathrm{C}\right) \delta 7.77(\mathrm{~d}, J=7.5 \mathrm{~Hz}, 2 \mathrm{H}, \mathrm{Ar} \mathrm{H})$, 
$7.61(\mathrm{~d}, J=7.5 \mathrm{~Hz}, 2 \mathrm{H}, \mathrm{Ar} \mathrm{H}), 7.41$ (t, $J=7.2 \mathrm{~Hz}, 2 \mathrm{H}, \mathrm{Ar} \mathrm{H}), 7.34-7.25$ (m, 6H, Ar H), 6.65 (s, 1H, FMOCNH), 5.88 (br s, $1 \mathrm{H}$, hydrazine NH), $5.40($ br m, $1 \mathrm{H}, \mathrm{CHN}), 4.54\left(\mathrm{~d}, J=6.5 \mathrm{~Hz}, 2 \mathrm{H}, \mathrm{CH}_{2} \mathrm{O}\right), 4.27\left(\mathrm{t}, J=6.5 \mathrm{~Hz}, 1 \mathrm{H}, \mathrm{CHCH}_{2} \mathrm{O}\right), 1.51(\mathrm{~d}, J=7.2 \mathrm{~Hz}$,

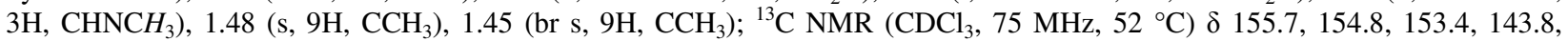
$141.5,137.0,128.0,127.8,127.1,124.9,120.0,119.0,81.3,81.0,66.9,55.3,47.3,28.3,28.2,16.9$; IR $v 3313(\mathrm{~m}), 3066(\mathrm{w})$, $2979(\mathrm{~m}), 2935(\mathrm{w}), 1702(\mathrm{~s}), 1601(\mathrm{~m}), 1535(\mathrm{~s}), 1478(\mathrm{~m}), 1451(\mathrm{~m}), 1393(\mathrm{~s}), 1368(\mathrm{~s}), 1316(\mathrm{~s}), 1222(\mathrm{~s}), 1166(\mathrm{~s}), 1106(\mathrm{w})$, $1086(\mathrm{~m}), 1055$ (m), 1018 (w), 993 (w), $910(\mathrm{~m}), 841(\mathrm{w}), 758(\mathrm{~m}), 737$ (s), $668(\mathrm{w}), 648(\mathrm{w}), 622(\mathrm{w})$; MS (ESI) 596.0 (M+Na); Anal. calcd for $\mathrm{C}_{33} \mathrm{H}_{39} \mathrm{~N}_{3} \mathrm{O}_{6}$ : C, 69.09; H, 6.85; N, 7.32. Found: C, 69.12; H, 7.04; N, 7.08.

$N$-\{1-[1-(Toluene-4-sulfonyl)-1H-pyrrol-2-yl]-ethyl\}- $N$ '-(tert-butoxycarbonyl)hydrazine carboxylic acid tert-butyl ester (141)

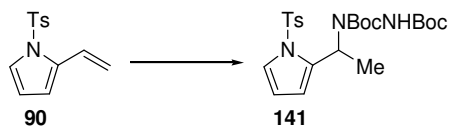

Following GP2 Hydrohydrazination product $141(168 \mathrm{mg}, 0.350 \mathrm{mmol}, 74 \%)$ was obtained as a colorless solid with vinylpyrrole 90 (96\% pure, $122 \mathrm{mg}, 0.474 \mathrm{mmol}, 1.00$ equiv) in $11 \mathrm{~h}$ with $2.5 \mathrm{~mol} \%$ catalyst 20 after purification by column chromatography (AcOEt/hexane 1:6). Following GP4 Hydrohydrazination product 141 (161 mg, 0.336 mmol, 70\%) was obtained as a colorless solid with vinylpyrrole 90 (96\% pure, $124 \mathrm{mg}, 0.481 \mathrm{mmol}, 1.00$ equiv) in $11 \mathrm{~h}$ with 1 mol\% catalyst 23 after purification by column chromatography (AcOEt/hexane 1:6). $\mathrm{R}_{\mathrm{f}}(\mathrm{AcOEt} / \mathrm{hexane} 1: 5)$ 0.25; Mp 58-60 ${ }^{\circ} \mathrm{C} ;{ }^{1} \mathrm{H} \mathrm{NMR}\left(\mathrm{CDCl} \mathrm{N}_{3}, 300\right.$ $\left.\mathrm{MHz}, 52{ }^{\circ} \mathrm{C}\right) \delta 7.68(\mathrm{~d}, J=8.1 \mathrm{~Hz}, 2 \mathrm{H}$, tosyl $\mathrm{CH}), 7.34(\mathrm{~m}, 1 \mathrm{H}$, pyrrole $\mathrm{H}), 7.28(\mathrm{~d}, J=8.1 \mathrm{~Hz}, 2 \mathrm{H}$, tosyl $\mathrm{CH}), 6.31(\mathrm{~m}, 1 \mathrm{H}$, pyrrole $\mathrm{H}), 6.22(\mathrm{t}, J=3.4 \mathrm{~Hz}, 1 \mathrm{H}$, pyrrole $\mathrm{H}), 5.57$ (br m, $2 \mathrm{H}, \mathrm{NH}$ and $\mathrm{CHN}), 2.40\left(\mathrm{~s}, 3 \mathrm{H}\right.$, tosyl $\left.\mathrm{CH}_{3}\right), 1.51\left(\mathrm{~s}, 9 \mathrm{H}, \mathrm{CCH}_{3}\right), 1.46$ $\left(\mathrm{m}, 3 \mathrm{H}, \mathrm{CHCH}_{3}\right), 1.41$ (br s, $\left.9 \mathrm{H}, \mathrm{CCH}_{3}\right) ;{ }^{13} \mathrm{C} \mathrm{NMR}\left(\mathrm{CDCl}_{3}, 75 \mathrm{MHz}, 52{ }^{\circ} \mathrm{C}\right) \delta 155.5,153.8,144.7,136.3,133.7,129.8,126.7$, 123.7, 115.4, 110.9, 81.0, 80.6, 49.9, 28.3, 28.2, 21.5, 18.4; IR v 3375 (w), 3153 (w), $2980(\mathrm{~m}), 2934$ (w), $1748(\mathrm{~m}), 1707$ (s), $1597(\mathrm{w}), 1479(\mathrm{~m}), 1456(\mathrm{~m}), 1393(\mathrm{~s}), 1368(\mathrm{~s}), 1310(\mathrm{~m}), 1254(\mathrm{~m}), 1176(\mathrm{~s}), 1155$ (s), $1094(\mathrm{~m}), 1080(\mathrm{~m}), 1048(\mathrm{~m}), 981(\mathrm{w})$, $912(\mathrm{w}), 874(\mathrm{w}), 858(\mathrm{w}), 814(\mathrm{w}), 756(\mathrm{w}), 733(\mathrm{~m}), 705(\mathrm{~m}), 686(\mathrm{~m}), 670(\mathrm{~m}), 590(\mathrm{~s}), 545(\mathrm{~m}) ; \mathrm{MS}(\mathrm{ESI}) 480.2(\mathrm{M}+\mathrm{H}), 502.2$ $(\mathrm{M}+\mathrm{Na}), 981.4(2 \mathrm{M}+\mathrm{Na})$; Anal. calcd for $\mathrm{C}_{23} \mathrm{H}_{33} \mathrm{~N}_{3} \mathrm{O}_{6} \mathrm{~S}$ : C, 57.60; H, 6.94; N, 8.76. Found: C, 57.68; H, 6.96; N, 8.63.

2-[1-(N,N'-Di-tert-butoxycarbonyl-hydrazino)-ethyl]-pyrrole-1-carboxylic acid tert-butyl ester (142)

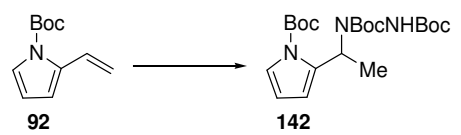

Following GP2 Hydrohydrazination product $142(135 \mathrm{mg}, 0.317 \mathrm{mmol}, 67 \%)$ was obtained as a colorless solid with vinylpyrrole 92 (92\% pure, $100 \mathrm{mg}, 0.476 \mathrm{mmol}, 1.00$ equiv) in $11 \mathrm{~h}$ with $2.5 \mathrm{~mol} \%$ catalyst 20 after purification by column chromatography (AcOEt/hexane 1:8). Following GP4 Hydrohydrazination product 142 (146 mg, 0.343 mmol, 73\%) was obtained as a colorless solid with vinylpyrrole 92 (92\% pure, $99 \mathrm{mg}, 0.47 \mathrm{mmol}, 1.0$ equiv) in $11 \mathrm{~h}$ with 1 mol\% catalyst 23 after purification by column chromatography (AcOEt/hexane 1:7). $\mathrm{R}_{\mathrm{f}}$ (AcOEt/hexane 1:5) 0.30; Mp 54-55 ${ }^{\circ} \mathrm{C} ;{ }^{1} \mathrm{H}$ NMR $\left(\mathrm{CDCl}{ }_{3}, 300\right.$ $\left.\mathrm{MHz}, 52{ }^{\circ} \mathrm{C}\right) \delta 7.18(\mathrm{dd}, J=3.1,1.9 \mathrm{~Hz}, 1 \mathrm{H}$, pyrrole $\mathrm{H}), 6.22(\mathrm{~m}, 1 \mathrm{H}$, pyrrole $\mathrm{H}), 6.07(\mathrm{t}, J=3.1 \mathrm{~Hz}, 1 \mathrm{H}$, pyrrole H), $5.92(\mathrm{br} \mathrm{m}$, $2 \mathrm{H}, \mathrm{NH}$ and $\mathrm{CHN}), 1.59$ (s, 9H, pyrrole-Boc $\left.\mathrm{CH}_{3}\right), 1.52\left(\mathrm{~d}, \mathrm{~J}=6.9 \mathrm{~Hz}, 3 \mathrm{H}, \mathrm{CHCH}_{3}\right), 1.46\left(\mathrm{~s}, 9 \mathrm{H}\right.$, hydrazine-Boc $\left.\mathrm{CH}_{3}\right), 1.42(\mathrm{br} \mathrm{s}$, 9H, hydrazine-Boc $\left.\mathrm{CH}_{3}\right) ;{ }^{13} \mathrm{C} \mathrm{NMR}\left(\mathrm{CDCl}_{3}, 75 \mathrm{MHz}, 52{ }^{\circ} \mathrm{C}\right) \delta$ 155.4, 154.2, 134.4, 121.9, 112.9, 109.5, 83.6, 80.6, 51.1, 28.3; 28.2, 28.0, 17.7; IR v $3335(\mathrm{w}), 3159(\mathrm{w}), 2979(\mathrm{~m}), 2935$ (m), 1748 (s), $1710(\mathrm{~s}), 1479(\mathrm{~m}), 1456(\mathrm{~m}), 1393$ (s), $1368(\mathrm{~s}), 1328$ (s), 1253 (s), $1167(\mathrm{~s}), 1137(\mathrm{~s}), 1088(\mathrm{w}), 1054(\mathrm{~m}), 1013(\mathrm{~m}), 987(\mathrm{~m}), 912(\mathrm{~m}), 883(\mathrm{w}), 851(\mathrm{~m}), 819(\mathrm{w}), 772(\mathrm{~m}), 733(\mathrm{~m})$, 648 (w), 597 (w), 564 (w), $486(w), 458$ (w); MS (ESI) $448.3(\mathrm{M}+\mathrm{Na})$; Anal. calcd for $\mathrm{C}_{21} \mathrm{H}_{35} \mathrm{~N}_{3} \mathrm{O}_{6}$ : C, 59.28; H, 8.29; N, 9.87. Found: C, 59.13; H, 8.34; N, 9.83.

$N$-\{1-[1-(Toluene-4-sulfonyl)-1H-indol-3-yl]-ethyl $\}-N$ '-(tert-butoxycarbonyl)hydrazine carboxylic acid tert-butyl ester (143)

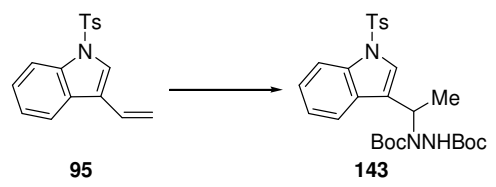

Following GP2 Hydrohydrazination product $143(220 \mathrm{mg}, 0.415 \mathrm{mmol}, 85 \%)$ was obtained as a colorless solid with vinylindole 95 (92\% pure, $157 \mathrm{mg}, 0.486 \mathrm{mmol}, 1.00$ equiv) in $11 \mathrm{~h}$ with $2.5 \mathrm{~mol} \%$ catalyst 20 after purification by column chromatography (pentane $/ \mathrm{CH}_{2} \mathrm{Cl}_{2} / \mathrm{Et}_{2} \mathrm{O}$ 1:1:0.2). Following GP4 Hydrohydrazination product 143 (126 mg, $0.238 \mathrm{mmol}, 51 \%$ ) was obtained as a colorless solid with vinylindole $\mathbf{9 5}(92 \%$ pure, $150 \mathrm{mg}, 0.464 \mathrm{mmol}, 1.00$ equiv) in $11 \mathrm{~h}$ with $1 \mathrm{~mol} \%$ catalyst $\mathbf{2 3}$ after purification by column chromatography (pentane/ $\left.\mathrm{CH}_{2} \mathrm{Cl}_{2} / \mathrm{Et}_{2} \mathrm{O} 1: 1: 0.2\right)$. $\mathrm{R}_{\mathrm{f}}\left(\mathrm{AcOEt} /\right.$ hexane 1:3) 0.30; $\mathrm{Mp} \mathrm{168-169}{ }^{\circ} \mathrm{C} ;{ }^{1} \mathrm{H} \mathrm{NMR}\left(\mathrm{CDCl}{ }_{3}, 300\right.$ $\left.\mathrm{MHz}, 52{ }^{\circ} \mathrm{C}\right) \delta 7.95(\mathrm{dm}, J=8.1 \mathrm{~Hz}, 1 \mathrm{H}$, indole $\mathrm{CH}), 7.75(\mathrm{~d}, J=8.4 \mathrm{~Hz}, 2 \mathrm{H}$, tosyl CH), $7.57($ br m, 1H, indole $\mathrm{CH}), 7.51(\mathrm{~s}, 1 \mathrm{H}$, indole $\mathrm{CH}$ ), 7.32-7.18 (m, $2 \mathrm{H}$, indole $\mathrm{CH}), 7.22(\mathrm{~d}, J=7.8 \mathrm{~Hz}, 2 \mathrm{H}$, tosyl $\mathrm{CH}), 5.70$ (br m, $2 \mathrm{H}, \mathrm{NH}$ and $\mathrm{CHN}), 2.34(\mathrm{~s}, 3 \mathrm{H}$, tosyl

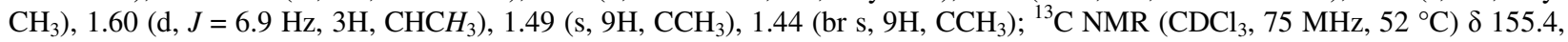
154.3, 144.7, 135.3, 135.3, 129.7, 126.6, 124.7, 124.5, 123.3, 122.7, 120.1, 113.6, 81.3, 80.9, 48.5, 28.3, 28.1, 21.5, 16.7; IR v 3354 (w), 3153 (w), 3053 (w), 2980 (m), 2934 (w), 1698 (s), 1598 (w), 1564 (w), 1494 (m), 1477 (m), 1450 (s), 1393 (s), 1368 (s), 1313 (s), 1279 (m), 1256 (m), 1214 (m), 1175 (s), 1129 (s), $1091(\mathrm{~s}), 1060$ (m), 1020 (m), 995 (w), 960 (m), 911 (m), 856 (w), 
843 (w), 813 (m), 734 (s), 704 (w), 670 (s), 648 (w), 618 (w), 578 (s), 538 (m); MS (ESI) 552.0 (M+Na); Anal. calcd for $\mathrm{C}_{27} \mathrm{H}_{35} \mathrm{~N}_{3} \mathrm{O}_{6} \mathrm{~S}: \mathrm{C}, 61.23 ; \mathrm{H}, 6.66 ; \mathrm{N}, 7.93$. Found: C, 61.13; H, 6.85; N, 7.78.

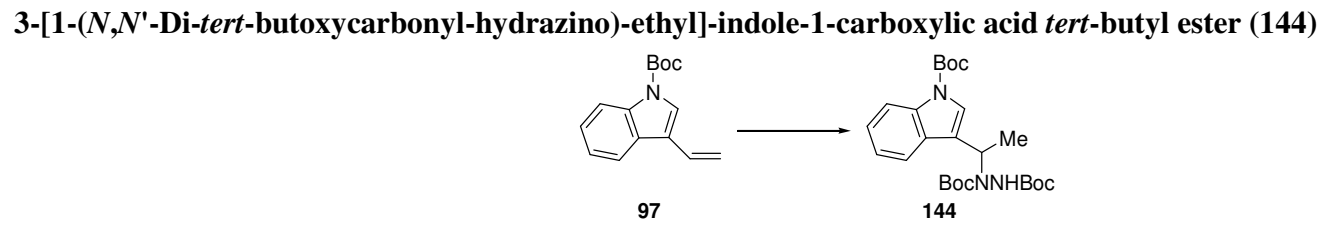

Following GP2 Hydrohydrazination product $144(172 \mathrm{mg}, 0.362 \mathrm{mmol}, 76 \%)$ was obtained as a colorless solid with vinylindole 97 (92\% pure, $126 \mathrm{mg}, 0.476 \mathrm{mmol}, 1.00$ equiv) in $11 \mathrm{~h}$ with $2.5 \mathrm{~mol} \%$ catalyst 20 after purification by column chromatography (AcOEt/hexane 1:7). Following GP4 Hydrohydrazination product 144 (169 mg, $0.355 \mathrm{mmol}, 73 \%)$ was obtained as a colorless solid with vinylindole 97 (92\% pure, $128 \mathrm{mg}, 0.484 \mathrm{mmol}, 1.00$ equiv) in $11 \mathrm{~h}$ with 1 mol\% catalyst 23 after purification by column chromatography (AcOEt/hexane 1:7). $\mathrm{R}_{\mathrm{f}}$ (AcOEt/hexane 1:5) $0.40 ; \mathrm{Mp} \mathrm{79-80}{ }^{\circ} \mathrm{C} ;{ }^{1} \mathrm{H} \mathrm{NMR}\left(\mathrm{CDCl}_{3}, 300 \mathrm{MHz}, 52{ }^{\circ} \mathrm{C}\right) \delta$ $8.12(\mathrm{~d}, J=8.1 \mathrm{~Hz}, 1 \mathrm{H}$, indole $\mathrm{CH}), 7.61(\mathrm{br} \mathrm{m}, 1 \mathrm{H}$, indole $\mathrm{CH}), 7.51(\mathrm{~s}, 1 \mathrm{H}$, indole $\mathrm{CH}), 7.33-7.18(\mathrm{~m}, 2 \mathrm{H}$, indole $\mathrm{CH}), 5.75(\mathrm{br}$ $\mathrm{m}, 2 \mathrm{H}, \mathrm{NH}$ and $\mathrm{CHN}), 1.69\left(\mathrm{~s}, 9 \mathrm{H}\right.$, indole-Boc $\left.\mathrm{CH}_{3}\right), 1.61\left(\mathrm{~d}, J=6.8 \mathrm{~Hz}, 3 \mathrm{H}, \mathrm{CHCH}_{3}\right), 1.52\left(\mathrm{~s}, 9 \mathrm{H}\right.$, hydrazine-Boc $\left.\mathrm{CH}_{3}\right), 1.44(\mathrm{br}$ s, 9H, hydrazine-Boc $\left.\mathrm{CH}_{3}\right) ;{ }^{13} \mathrm{C} \mathrm{NMR}\left(\mathrm{CDCl}_{3}, 75 \mathrm{MHz}, 52{ }^{\circ} \mathrm{C}\right) \delta 155.4,154.3,149.5,135.5,129.3,124.4,123.9,122.6,120.7$, 119.5, 115.1, 83.7, 81.1, 80.7, 48.7, 28.3, 28.3, 16.8; IR v 3326 (w), 3159 (w), 3053 (w), 2980 (s), 2935 (m), 1732 (s), 1609 (w), 1570 (w), 1478 (m), 1455 (s), 1369 (s), 1310 (s), 1256 (s), 1224 (s), 1160 (s), 1108 (s), 1064 (m), 1026 (m), 1010 (m), 973 (w), $911(\mathrm{~m}), 857(\mathrm{~m}), 768(\mathrm{~m}), 734(\mathrm{~s}), 648(\mathrm{w}), 582(\mathrm{w}), 457(\mathrm{w})$; MS (ESI) $498.2(\mathrm{M}+\mathrm{Na})$; Anal. calcd for $\mathrm{C}_{25} \mathrm{H}_{37} \mathrm{~N}_{3} \mathrm{O}_{6}$ : C, 63.14 ; H, 7.84; N, 8.84. Found: C, 63.05; H, 7.84; N, 8.66.

\section{$N$-(1-Methyl-1-phenyl-ethyl)- $N$ '-(tert-butoxycarbonyl)hydrazinecarboxylic acid tert-butyl ester (146)}

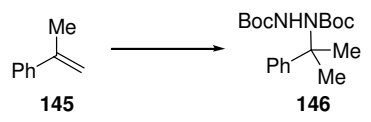

Following GP2 Hydrohydrazination product $146(155 \mathrm{mg}, 0.443 \mathrm{mmol}, 88 \%)$ was obtained as a viscous oil with $\alpha$-methyl styrene (145) (freshly filtered through neutral $\mathrm{Al}_{2} \mathrm{O}_{3}, 65 \mu \mathrm{L}, 0.50 \mathrm{mmol}, 1.0$ equiv) in $2 \mathrm{~h}$ with 5 mol\% catalyst after purification by column chromatography (AcOEt/hexane 1:10). $\mathrm{R}_{\mathrm{f}}$ (AcOEt/hexane 1:5) $0.33 ;{ }^{1} \mathrm{H}$ NMR $\left(\mathrm{CDCl}_{3}, 300 \mathrm{MHz}, 52{ }^{\circ} \mathrm{C}\right) \delta 7.45(\mathrm{~d}, J=$ $7.5 \mathrm{~Hz}, 2 \mathrm{H}, \mathrm{Ar} \mathrm{H}), 7.27$ (t, $J=7.5 \mathrm{~Hz}, 2 \mathrm{H}, \mathrm{Ar} \mathrm{H}), 7.16(\mathrm{t}, J=7.5 \mathrm{~Hz}, 2 \mathrm{H}, \mathrm{Ar} \mathrm{H}), 6.42(\mathrm{br} \mathrm{s}, 1 \mathrm{H}, \mathrm{NH}), 1.71\left(\mathrm{~s}, 3 \mathrm{H}, \mathrm{CNCH}_{3}\right), 1.56$ $\left(\mathrm{s}, 3 \mathrm{H}, \mathrm{CNCH}_{3}\right), 1.52\left(\mathrm{~s}, 9 \mathrm{H}, \mathrm{CCH}_{3}\right), 1.16\left(\mathrm{~s}, 9 \mathrm{H}, \mathrm{CCH}_{3}\right) ;{ }^{13} \mathrm{C} \mathrm{NMR}\left(\mathrm{CDCl}_{3}, 300 \mathrm{MHz}, 52{ }^{\circ} \mathrm{C}\right) \delta 156.6,154.6,149.0,127.9$, 125.9, 124.6, 81.0, 63.8, 28.7, 28.3, 28.0, 27.9; IR v 3269 (m), 3089 (w), $3061(\mathrm{w}), 2979$ (s), 2933 (m), 2175 (w), $1949(\mathrm{w}), 1712$ (s), $1602(\mathrm{w}), 1495(\mathrm{~m}), 1479(\mathrm{~m}), 1450(\mathrm{~m}), 1390$ (s), 1367 (s), 1341 (s), 1250 (s), 1158 (s), $1114(\mathrm{~m}), 1097$ (m), 1078 (m), 1047 (m), 1019 (m), 911 (m), 857 (w), $764(\mathrm{~m}), 734$ (m), 700 (s), 648 (w), 602 (w); HRMS (ESI) calcd for $\mathrm{C}_{19} \mathrm{H}_{30} \mathrm{~N}_{2} \mathrm{O}_{4} \mathrm{Na}^{+}(\mathrm{M}+\mathrm{Na})$ 373.2098, found 373.2103; Anal. calcd for $\mathrm{C}_{19} \mathrm{H}_{30} \mathrm{~N}_{2} \mathrm{O}_{4}$ : C, 65.12; H, 8.63; N, 7.99. Found: C, 65.23; H, 8.50; N, 8.10.

\section{$N$-(1-Phenyl-propyl)- $N$ '-(tert-butoxycarbonyl)hydrazinecarboxylic acid tert-butyl ester (148)}

$$
\text { Ph } \overbrace{147} \longrightarrow{ }_{148}^{\text {BocNHNBoc }} \mathrm{Me}
$$

Following GP1 Hydrohydrazination product 148 (154 mg, $0.440 \mathrm{mmol}, 88 \%)$ was obtained as a colorless solid with $\beta$-methylstyrene (147) $(65 \mu \mathrm{L}, 0.50 \mathrm{mmol} 1.0$ equiv) in $3 \mathrm{~h}$ with $5 \mathrm{~mol} \%$ catalyst $\mathbf{2 0}$ after purification by column chromatography (AcOEt/hexane 1:10). Following GP2 Hydrohydrazination product 148 (165 mg, $0.471 \mathrm{mmol}, 94 \%)$ was obtained as a colorless solid with $\beta$-methyl-styrene (147) $(65 \mu \mathrm{L}, 0.50 \mathrm{mmol} 1.0$ equiv) in $2 \mathrm{~h}$ with $2 \mathrm{~mol} \%$ catalyst 23 after purification by column chromatography (AcOEt/hexane 1:10). $\mathrm{R}_{\mathrm{f}}\left(\mathrm{AcOEt} /\right.$ hexane 1:5) $0.30 ; \mathrm{Mp} 97-98{ }^{\circ} \mathrm{C} ;{ }^{1} \mathrm{H} \mathrm{NMR}\left(\mathrm{CDCl}_{3}, 300 \mathrm{MHz}, 52{ }^{\circ} \mathrm{C}\right) \delta 7.33-$ 7.22 (m, 5H, Ar H), 5.78 (br s, 1H, NH), 5.12 (br m, 1H, CHN), 2.11-1.81 (m, 2H, $\left.\mathrm{CH}_{2}\right), 1.47$ (s, 9H, $\left.\mathrm{CCH}_{3}\right), 1.43($ br s, $9 \mathrm{H}$, $\left.\mathrm{CCH}_{3}\right), 0.96\left(\mathrm{t}, J=7.2 \mathrm{~Hz}, 3 \mathrm{H}, \mathrm{CH}_{2} \mathrm{CH}_{3}\right) ;{ }^{13} \mathrm{C} \mathrm{NMR}\left(\mathrm{CDCl}_{3}, 75 \mathrm{MHz}, 52{ }^{\circ} \mathrm{C}\right) \delta 155.5,154.9,139.6,128.2,128.2,127.4,81.2$, 80.8, 62.3, 28.4, 28.2, 24.0, 11.3; IR v $3221(\mathrm{~m}), 3151(\mathrm{~m}), 2970(\mathrm{~m}), 2935(\mathrm{~m}), 2878(\mathrm{w}), 1714(\mathrm{~s}), 1694(\mathrm{~s}), 1497(\mathrm{~m}), 1478(\mathrm{~m})$, $1456(\mathrm{~m}), 1404$ (s), 1366 (s), 1319 (s), 1283 (m), 1258 (m), 1169 (s), 1138 (s), 1082 (m), 1050 (m), 1017 (m), 942 (m), 907 (w), $875(\mathrm{~m}), 767(\mathrm{~m}), 749(\mathrm{~m}), 698(\mathrm{~s}), 632(\mathrm{~m}), 603$ (w), 521 (w); HRMS (ESI) calcd for $\mathrm{C}_{19} \mathrm{H}_{30} \mathrm{~N}_{2} \mathrm{O}_{4} \mathrm{Na}^{+}$(M+Na) 373.2098, found 373.2090;Anal. calcd for $\mathrm{C}_{19} \mathrm{H}_{30} \mathrm{~N}_{2} \mathrm{O}_{4}$ : C, 65.12; H, 8.63; N, 7.99. Found: C, 64.88; H, 8.43; N, 8.03.

\section{$N$-(3-Hydroxy-1-phenyl-propyl)- $N$ '-(tert-butoxycarbonyl)hydrazinecarboxylic acid tert-butyl ester (150)}

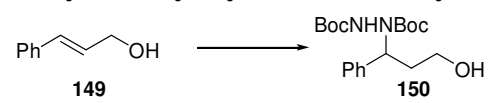

Following GP1 Hydrohydrazination product 150 (167 mg, $0.456 \mathrm{mmol}, 91 \%)$ was obtained as a colorless solid with cinnamoyl alcohol (149) (67 mg, $0.50 \mathrm{mmol}, 1.0$ equiv) in $1 \mathrm{~h}$ with $5 \mathrm{~mol} \%$ catalyst 20 after purification by column chromatography (AcOEt/hexane 1:5-1:1). $\mathrm{R}_{\mathrm{f}}\left(\mathrm{AcOEt} /\right.$ hexane 1:2) 0.20; $\mathrm{Mp} \mathrm{102-104}{ }^{\circ} \mathrm{C} ;{ }^{1} \mathrm{H} \mathrm{NMR}\left(\mathrm{CDCl}_{3}, 300 \mathrm{MHz}, 52{ }^{\circ} \mathrm{C}\right) \delta 7.31-7.21(\mathrm{~m}, 5 \mathrm{H}$, Ar H), 5.96 (br s, 1H, NH), 5.40 (br s, $1 \mathrm{H}, \mathrm{CHN}$ ), 3.89 (br s, $\left.1 \mathrm{H}, \mathrm{CH}_{2} \mathrm{OH}\right), 3.73$ (br s, $\left.1 \mathrm{H}, \mathrm{CH}_{2} \mathrm{OH}\right), 3.18\left(\right.$ br s, $\left.1 \mathrm{H}, \mathrm{CH} \mathrm{H}_{2} \mathrm{OH}\right), 2.22$ (br m, 1H, CHNCH $\left.\mathrm{CH}_{2}\right), 1.99$ (br m, $\left.1 \mathrm{H}, \mathrm{CHNCH}_{2}\right), 1.46\left(\mathrm{~s}, 9 \mathrm{H}, \mathrm{CCH}_{3}\right), 1.42$ (br s, 9H, $\left.\mathrm{CCH}_{3}\right) ;{ }^{13} \mathrm{C} \mathrm{NMR}\left(\mathrm{CDCl}_{3}, 75 \mathrm{MHz}^{2} 52{ }^{\circ} \mathrm{C}\right)$ $\delta \quad 155.6,155.3,139.3,128.2,127.9,127.5,81.6,81.1,59.7,57.4,33.7,28.3,28.1$; IR v $3425(\mathrm{w}, \mathrm{sh}), 3306(\mathrm{~m}), 3032(\mathrm{w}), 2979$ (m), 2933 (m), 1703 (s), 1478 (m), 1455 (m), 1393 (s), 1368 (s), 1324 (s), 1253 (s), 1162 (s), 1050 (m), 912 (m), 863 (w), 734 
(m), $700(\mathrm{~m})$; MS (ESI) $367.1(\mathrm{M}+\mathrm{H}), 389.2(\mathrm{M}+\mathrm{Na}), 755.1(2 \mathrm{M}+\mathrm{Na})$; Anal. calcd for $\mathrm{C}_{19} \mathrm{H}_{30} \mathrm{~N}_{2} \mathrm{O}_{5}$ : C, 62.27; H, 8.25; N, 7.64. Found: C, 62.20; H, 8.18; N, 7.60.

$N$-(1H-Inden-1-yl)-N'-(tert-butoxycarbonyl)hydrazinecarboxylic acid tert-butyl ester (152)

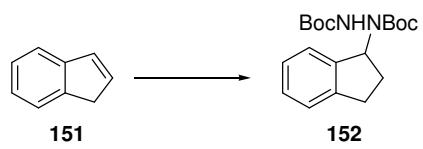

Following GP1 Hydrohydrazination product $152(163 \mathrm{mg}, 0.468 \mathrm{mmol}, 94 \%)$ was obtained as a colorless solid with indene (151) $(59 \mu \mathrm{L}, 0.50 \mathrm{mmol}, 1.0$ equiv) in $1 \mathrm{~h}$ with $5 \mathrm{~mol} \%$ catalyst 20 after purification by column chromatography (AcOEt/hexane 1:10). $\mathrm{R}_{\mathrm{f}}$ (AcOEt/hexane 1:5) 0.35; Mp 93-95 ${ }^{\circ} \mathrm{C} ;{ }^{1} \mathrm{H} \mathrm{NMR}\left(\mathrm{CDCl}_{3}, 300 \mathrm{MHz}, 52{ }^{\circ} \mathrm{C}\right) \delta$ 7.22-7.14 (m, 4H, Ar H), $5.93(\mathrm{br} \mathrm{s}, 1 \mathrm{H}$, $\mathrm{NH}), 5.76($ br s, $1 \mathrm{H}, \mathrm{CHN}), 3.00\left(\mathrm{~m}, 1 \mathrm{H}, \mathrm{CH}_{2}\right), 2.81\left(\mathrm{~m}, 1 \mathrm{H}, \mathrm{CH}_{2}\right), 2.38\left(\mathrm{~m}, 1 \mathrm{H}, \mathrm{CH}_{2}\right), 2.17\left(\mathrm{~m}, 1 \mathrm{H}, \mathrm{CH}_{2}\right), 1.51\left(\mathrm{~s}, 9 \mathrm{H}, \mathrm{CCH}_{3}\right)$, 1.42 (br s, 9H, $\left.\mathrm{CCH}_{3}\right) ;{ }^{13} \mathrm{C} \mathrm{NMR}\left(\mathrm{CDCl}_{3}, 75 \mathrm{MHz}, 52{ }^{\circ} \mathrm{C}\right) \delta 155.5,155.1,144.1,141.2,127.9,126.4,124.8,124.2,81.3,80.9$, 63.1, 30.5, 29.0, 28.3, 28.1; IR v $3314(\mathrm{~m}), 3072(\mathrm{w}), 2979(\mathrm{~m}), 2933(\mathrm{~m}), 1705(\mathrm{~s}), 1480(\mathrm{~m}), 1458(\mathrm{~m}), 1393(\mathrm{~s}), 1367(\mathrm{~s}), 1332$ (m), $1297(\mathrm{~m}), 1246$ (s), $1161(\mathrm{~s}), 1052(\mathrm{~m}), 1020(\mathrm{w}), 940(\mathrm{w}), 920(\mathrm{w}), 854(\mathrm{w}), 830(\mathrm{w}), 758(\mathrm{~m}), 737(\mathrm{~m}), 647(\mathrm{w}), 591(\mathrm{w})$; MS (ESI) $349.3(\mathrm{M}+\mathrm{H}), 371.1(\mathrm{M}+\mathrm{Na})$; Anal. calcd for $\mathrm{C}_{19} \mathrm{H}_{28} \mathrm{~N}_{2} \mathrm{O}_{4}$ : C, 65.49; H, 8.10; N, 8.04. Found: C, 65.31; H, 8.13; N, 7.87 .

\section{$N$-(1-Phenyl-cyclohexyl)-N'-(tert-butoxycarbonyl)hydrazinecarboxylic acid tert-butyl ester (154)}

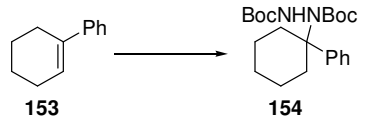

Following GP1 Hydrohydrazination product $154(157 \mathrm{mg}, 0.403 \mathrm{mmol}, 80 \%)$ was obtained as a colorless viscous oil with 1 phenyl cyclohexene (153) $(80 \mu \mathrm{L}, 0.50 \mathrm{mmol}, 1.0$ equiv), phenylsilane ( $97 \mu \mathrm{L}, 0.75 \mathrm{mmol}, 1.5$ equiv) and di-tert-butyl azodicarboxylate (2) $(0.26 \mathrm{~g}, 1.1 \mathrm{mmol}, 2.2$ equiv) in $8 \mathrm{~h}$ with $5 \mathrm{~mol} \%$ catalyst 20 after purification by column chromatography (AcOEt/hexane 1:10). $\mathrm{R}_{\mathrm{f}}(\mathrm{AcOEt} / \mathrm{hexane} 1: 5)$ 0.35; ${ }^{1} \mathrm{H} \mathrm{NMR}\left(\mathrm{CDCl}_{3}, 300 \mathrm{MHz}, 52{ }^{\circ} \mathrm{C}\right) \delta 7.46(\mathrm{~d}, J=7.5 \mathrm{~Hz}, 2 \mathrm{H}, \mathrm{Ar} \mathrm{H}), 7.27(\mathrm{~m}$, $2 \mathrm{H}, \mathrm{Ar} \mathrm{H}), 7.17(\mathrm{~m}, 1 \mathrm{H}, \mathrm{Ar} \mathrm{H}), 6.11(\mathrm{br} \mathrm{s}, 1 \mathrm{H}, \mathrm{NH}), 2.52\left(\mathrm{~m}, 1 \mathrm{H}, \mathrm{CH}_{2}\right), 2.35-2.06\left(\mathrm{~m}, 3 \mathrm{H}, \mathrm{CH}_{2}\right), 1.83-1.34\left(\mathrm{~m}, 6 \mathrm{H}, \mathrm{CH}_{2}\right), 1.47(\mathrm{~s}$, $\left.9 \mathrm{H}, \mathrm{CCH}_{3}\right), 1.26\left(\mathrm{~s}, 9 \mathrm{H}, \mathrm{CCH}_{3}\right) ;{ }^{13} \mathrm{C} \mathrm{NMR}\left(\mathrm{CDCl}_{3}, 75 \mathrm{MHz}, 52{ }^{\circ} \mathrm{C}\right) \delta 156.3,155.0,145.7,127.9,126.4,126.4,81.0,80.8,66.3$, 36.1, 35.6, 28.2, 28.0, 25.7, 23.0, 22.7; IR v 3263 (m), 3156 (w), 3093 (w), 3059 (w), 2977 (s), $2933(\mathrm{~s}), 2866$ (m), 1705 (s), 1602 (w), 1479 (m), 1454 (s), 1392 (s), 1367 (s), 1332 (s), 1248 (s), 1163 (s), 1108 (m), 1085 (m), 1047 (m), 1015 (m), 911 (m), 884 $(\mathrm{w}), 857(\mathrm{w}), 754(\mathrm{~m}), 734(\mathrm{~s}), 700(\mathrm{~m}), 647(\mathrm{w}), 613(\mathrm{w}), 576(\mathrm{w}), 529(\mathrm{w})$; MS (ESI) 413.2 (M+Na); Anal. calcd for $\mathrm{C}_{22} \mathrm{H}_{34} \mathrm{~N}_{2} \mathrm{O}_{4}$ : C, 67.66; H, 8.78; N, 7.17. Found: C, 67.67; H, 8.92; N, 7.45.

$N$-(1-Methyl-1-pyridin-2-yl-ethyl)- $N$ '-(tert-butoxycarbonyl)hydrazinecarboxylic acid tert-butyl ester (155)

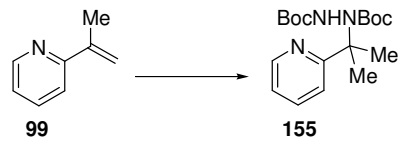

Following GP2 Hydrohydrazination product 155 (106 mg, $0.302 \mathrm{mmol}, 60 \%)$ was obtained as a colorless gel with vinylpyridine 99 (60 mg, $0.50 \mathrm{mmol}, 1.0$ equiv) in $5 \mathrm{~h}$ with $2.5 \mathrm{~mol} \%$ catalyst 20 after purification by column chromatography (AcOEt/hexane 1:4). Following GP4 Hydrohydrazination product 155 (75 mg, $0.21 \mathrm{mmol}, 42 \%$ ) was obtained as a colorless gel with vinylpyridine $99(60 \mathrm{mg}, 0.50 \mathrm{mmol}, 1.0$ equiv) in $5 \mathrm{~h}$ with $1 \mathrm{~mol} \%$ catalyst 23 after purification by column chromatography (AcOEt/hexane 1:4). ${ }^{1} \mathrm{H}$ NMR $\left(\mathrm{CDCl}_{3}, 300 \mathrm{MHz}, 52{ }^{\circ} \mathrm{C}\right) \delta 8.42(\mathrm{~m}, 1 \mathrm{H}$, pyridine $\mathrm{CH}), 7.66-7.54(\mathrm{~m}, 2 \mathrm{H}, \mathrm{pyridine} \mathrm{CH}), 7.04(\mathrm{~m}$, $1 \mathrm{H}$, pyridine $\mathrm{CH}), 6.65$ (br s, $1 \mathrm{H}, \mathrm{NH}), 1.74\left(\mathrm{~s}, 3 \mathrm{H}, \mathrm{C}\left(\mathrm{CH}_{3}\right)_{2}\right), 1.54\left(\mathrm{~s}, 3 \mathrm{H}, \mathrm{C}\left(\mathrm{CH}_{3}\right)_{2}\right), 1.48\left(\mathrm{~s}, 9 \mathrm{H}, \mathrm{OC}\left(\mathrm{CH}_{3}\right)_{3}\right), 1.15(\mathrm{~s}, 9 \mathrm{H}$, $\left.\mathrm{OC}\left(\mathrm{CH}_{3}\right)_{3}\right) ;{ }^{13} \mathrm{C} \mathrm{NMR}\left(\mathrm{CDCl}_{3}, 75 \mathrm{MHz}, 25{ }^{\circ} \mathrm{C}\right) \delta 166.5,156.3,154.7,147.7,136.1,121.0,119.9,80.9,80.5,65.3,28.3,27.9$, 27.2; IR v 3286 (m), 3053 (w), 2980 (s), 2934 (s), 1715 (s), 1593 (s), 1571 (m), 1480 (s), 1430 (s), 1368 (s), 1249 (s), 1157 (s), $1082(\mathrm{~m}), 1048(\mathrm{~m}), 1020(\mathrm{~m}), 995(\mathrm{w}), 911(\mathrm{~m}), 858(\mathrm{w}), 839(\mathrm{w}), 788(\mathrm{~m}), 733$ (s), $647(\mathrm{~m}), 605$ (s); MS (ESI) 374.4 (M+Na); Anal. calcd for $\mathrm{C}_{18} \mathrm{H}_{29} \mathrm{~N}_{3} \mathrm{O}_{4}$ : C, 61.52; H, 8.32; N, 11.96. Found: C, 61.44; H, 8.36; N, 11.86 .

$N$-(1-pyridin-2-yl-propyl)- $N$ '-(tert-butoxycarbonyl)hydrazinecarboxylic acid tert-butyl ester (156)

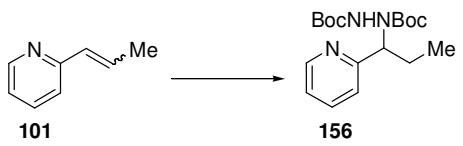

Following GP2 Hydrohydrazination product 156 (96 mg, $0.27 \mathrm{mmol}, 54 \%$ ) was obtained as a colorless gel with vinylpyridine 101 (60 mg, $0.50 \mathrm{mmol}, 1.0$ equiv) in $5 \mathrm{~h}$ with $2.5 \mathrm{~mol} \%$ catalyst 20 after purification by column chromatography (AcOEt/hexane 1:2). Following GP4 Hydrohydrazination product 156 (94 $\mathrm{mg}, 0.27 \mathrm{mmol}, 54 \%)$ was obtained as a colorless gel with vinylpyridine 101 (60 mg, $0.50 \mathrm{mmol}, 1.0$ equiv) in $5 \mathrm{~h}$ with $1 \mathrm{~mol} \%$ catalyst 23 after purification by column chromatography (AcOEt/hexane 1:4). $\mathrm{R}_{\mathrm{f}}(\mathrm{AcOEt} /$ hexane $1: 2) 0.40 ;{ }^{1} \mathrm{H} \mathrm{NMR}\left(\mathrm{CDCl}_{3}, 300 \mathrm{MHz}, 52{ }^{\circ} \mathrm{C}\right) \delta 8.50(\mathrm{dm}, J=4.8 \mathrm{~Hz}, 1 \mathrm{H}, \mathrm{pyridine} \mathrm{CH})$, $7.61(\mathrm{td}, J=7.7,1.8 \mathrm{~Hz}, 1 \mathrm{H}$, pyridine $\mathrm{CH}), 7.23(\mathrm{~m}, 1 \mathrm{H}$, pyridine $\mathrm{CH}), 7.14(\mathrm{ddd}, J=7.5,4.9,1.1 \mathrm{~Hz}, 1 \mathrm{H}$, pyridine $\mathrm{CH}), 6.84(\mathrm{br}$ s, $1 \mathrm{H}, \mathrm{NH}), 5.11(\mathrm{br} \mathrm{m}, 1 \mathrm{H}, \mathrm{CHN}), 2.04-1.92\left(\mathrm{~m}, 2 \mathrm{H}, \mathrm{CH}_{2}\right), 1.46\left(\mathrm{~s}, 9 \mathrm{H}, \mathrm{OC}\left(\mathrm{CH}_{3}\right)_{3}\right), 1.41\left(\mathrm{~s}, 9 \mathrm{H}, \mathrm{OC}\left(\mathrm{CH}_{3}\right)_{3}\right), 1.02(\mathrm{t}, J=7.3 \mathrm{~Hz}$, $\left.\mathrm{CH}_{2} \mathrm{CH}_{3}\right) ;{ }^{13} \mathrm{C} \mathrm{NMR}\left(\mathrm{CDCl}_{3}, 75 \mathrm{MHz}, 25{ }^{\circ} \mathrm{C}\right) \delta 160.5,156.0,155.0,148.9,136.1,122.0,122.0,81.0,80.2,62.8,28.1,28.0,24.8$, 11.1; IR $\vee 3306(w), 3054(w), 2978$ (m), 2934 (m), 2879 (w), 1746 (s), $1710(\mathrm{~s}), 1642$ (w), 1592 (m), 1572 (m), 1548 (w), 1512 
(w), $1478(\mathrm{~m}), 1461(\mathrm{~m}), 1435$ (m), 1392 (s), 1367 (s), 1333 (m), 1307 (m), 1254 (s), 1163 (s), $1104(\mathrm{~m}), 1050$ (m), 1017 (m), 996 (w), $940(w), 920(w), 858(w), 758(m), 647(w), 602(w), 534(w) ;$ MS (ESI) $374.2(\mathrm{M}+\mathrm{Na})$; Anal. calcd for $\mathrm{C}_{18} \mathrm{H}_{29} \mathrm{~N}_{3} \mathrm{O}_{4}$ : C, 61.52; H, 8.32; N, 11.96. Found: C, 61.55; H, 8.44; N, 11.78 .

$N$-(1-Methyl-1-thiophen-2-yl-ethyl)- $N$ '-(tert-butoxycarbonyl)hydrazinecarboxylic acid tert-butyl ester (157)

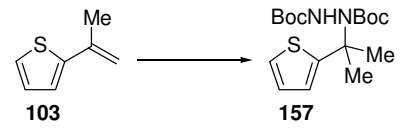

Following GP2 Hydrohydrazination product 157 (103 mg, $0.289 \mathrm{mmol}, 58 \%)$ was obtained as a colorless gel with vinylthiophene 103 (62 mg, $0.50 \mathrm{mmol}, 1.0$ equiv) in $7 \mathrm{~h}$ with $2.5 \mathrm{~mol} \%$ catalyst 20 after purification by column chromatography (AcOEt/hexane 1:6). Following GP4 Hydrohydrazination product $157(110 \mathrm{mg}, 0.309 \mathrm{mmol}, 62 \%)$ was obtained as a colorless gel with vinylthiophene $103(62 \mathrm{mg}, 0.50 \mathrm{mmol}, 1.0$ equiv) in $7 \mathrm{~h}$ with 1 mol\% catalyst 23 after purification by column chromatography (AcOEt/hexane 1:6). ${ }^{1} \mathrm{H} \mathrm{NMR}\left(\mathrm{CDCl}_{3}, 300 \mathrm{MHz}, 25{ }^{\circ} \mathrm{C}\right) \delta 7.11(\mathrm{~m}, 1 \mathrm{H}$, thiophene $\mathrm{CH}), 6.95-6.87(\mathrm{~m}, 2 \mathrm{H}$, thiophene $\mathrm{CH}), 6.35-6.00$ (br s, $1 \mathrm{H}, \mathrm{NH}), 1.89\left(\mathrm{~s}, 3 \mathrm{H}, \mathrm{C}\left(\mathrm{CH}_{3}\right)_{2}\right), 1.65\left(\mathrm{~s}, 3 \mathrm{H}, \mathrm{C}\left(\mathrm{CH}_{3}\right)_{2}\right), 1.49\left(\mathrm{~s}, 9 \mathrm{H}, \mathrm{OC}(\mathrm{CH})_{3}\right), 1.29(\mathrm{~s}, 9 \mathrm{H}$, $\left.\mathrm{OC}\left(\mathrm{CH}_{3}\right)_{3}\right) ;{ }^{13} \mathrm{C} \mathrm{NMR}\left(\mathrm{CDCl}_{3}, 75 \mathrm{MHz}, 25{ }^{\circ} \mathrm{C}\right) \delta 156.0,154.1,153.7,126.0,122.6,122.1,81.3,80.8,62.0,30.5,29.0,28.3,28.1$; IR v $3273(\mathrm{~m}), 2980(\mathrm{~s}), 2933(\mathrm{~m}), 1714(\mathrm{~s}), 1479(\mathrm{~m}), 1456(\mathrm{~m}), 1391(\mathrm{~s}), 1367(\mathrm{~s}), 1249$ (s), $1156(\mathrm{~s}), 1106(\mathrm{~m}), 1080$ (m), 1047 $(\mathrm{m}), 1018(\mathrm{~m}), 911(\mathrm{~m}), 853(\mathrm{~m}), 831(\mathrm{w}), 734(\mathrm{~m}), 693(\mathrm{~m}), 647(\mathrm{w}), 621(\mathrm{w})$; MS (ESI) 379.4 (M+Na); Anal. calcd for $\mathrm{C}_{17} \mathrm{H}_{28} \mathrm{~N}_{2} \mathrm{O}_{4} \mathrm{~S}: \mathrm{C}, 57.28 ; \mathrm{H}, 7.92 ; \mathrm{N}, 7.86$. Found: C, 57.05; H, 8.08; N, 7.74.

\section{$N$-(1-Benzothiazol-2-yl-1-methyl-ethyl)- $N$ '-(ethoxycarbonyl)hydrazinecarboxylic acid ethyl ester (158)}

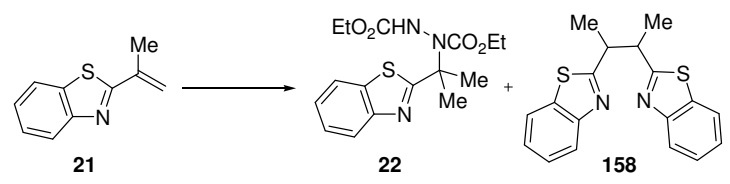

Following GP2 (slightly modified) The hydrohydrazination reaction of 21 under standard conditions GP2 or GP4 with di-tertbutyl azodicarboxylate (2) was not successful due to extensive dimerization of $\mathbf{2 1}$. However, hydrohydrazination product $\mathbf{2 2}$ (95 $\mathrm{mg}, 0.27 \mathrm{mmol}, 54 \%$ ) was obtained as a colorless solid with vinyl benzothiazole 21 ( $88 \mathrm{mg}, 0.50$ mmol, 1.0 equiv), diethyl azodicarboxylate $(0.24 \mathrm{~mL}, 1.5 \mathrm{mmol}, 3.0$ equiv) and phenylsilane $(0.13 \mathrm{~mL}, 1.0 \mathrm{mmol}, 2.0$ equiv) in $2 \mathrm{~d}$ with $0.25 \mathrm{~mol} \%$ catalyst 20 after purification by column chromatography (AcOEt/hexane 1:3). About 30\% of dimer $\mathbf{1 5 8}$ was still detected in the crude mixture via ${ }^{1} H$-NMR. $\mathrm{R}_{\mathrm{f}}(\mathrm{AcOEt} /$ hexane $1: 2)$ 0.25; $\mathrm{Mp} 43-45{ }^{\circ} \mathrm{C} ;{ }^{1} \mathrm{H}$ NMR $\left(\mathrm{CDCl}_{3}, 300 \mathrm{MHz}, 52{ }^{\circ} \mathrm{C}\right) \delta 7.95(\mathrm{dd}, J=8.1,0.6 \mathrm{~Hz}$, $1 \mathrm{H}, \operatorname{Ar~H}), 7.82$ (dt, $J=8.1,0.6 \mathrm{~Hz}, 1 \mathrm{H}, \mathrm{Ar} \mathrm{H}), 7.43$ (m, 1H, Ar H), 7.33 (m, 1H, Ar H), 6.77 (br s, 1H, NH), 4.35-4.20 (m, 2H, $\left.\mathrm{CH}_{2}\right), 4.10-4.00\left(\mathrm{~m}, 2 \mathrm{H}, \mathrm{CH}_{2}\right), 2.03\left(\mathrm{~s}, 3 \mathrm{H}, \mathrm{C}\left(\mathrm{CH}_{3}\right)_{2}\right), 1.75\left(\mathrm{~s}, 3 \mathrm{H}, \mathrm{C}\left(\mathrm{CH}_{3}\right)_{2}\right), 1.36-1.26\left(\mathrm{~m}, 3 \mathrm{H}, \mathrm{CH}_{2} \mathrm{CH}_{3}\right), 1.12-1.03(\mathrm{~m}, 3 \mathrm{H}$, $\left.\mathrm{CH}_{2} \mathrm{CH}_{3}\right) ;{ }^{13} \mathrm{C} \mathrm{NMR}\left(\mathrm{CDCl}_{3}, 75 \mathrm{MHz}, 52{ }^{\circ} \mathrm{C}\right) \delta 178.0,156.8,155.3,152.6,135.2,125.6,124.6,122.8,121.4,65.0,62.4,62.1$, 28.5, 27.4, 14.5, 14.2; IR v 3299 (m), $3063(\mathrm{w}), 2986(\mathrm{~m}), 2938(\mathrm{w}), 1723(\mathrm{~s}), 1519(\mathrm{~m}), 1456(\mathrm{~m}), 1440(\mathrm{~m}), 1402(\mathrm{~m}), 1377(\mathrm{~m})$, 1339 (m), 1314 (m), 1255 (s), 1190 (m), $1174(\mathrm{~m}), 1098(\mathrm{~s}), 1057$ (m), 1015 (m), 915 (m), 865 (w), 824 (w), $762(\mathrm{~m}), 731$ (m), 647 (w), $522(\mathrm{w})$; MS (ESI) $374.1(\mathrm{M}+\mathrm{Na})$; Anal. calcd for $\mathrm{C}_{16} \mathrm{H}_{21} \mathrm{~N}_{3} \mathrm{O}_{4} \mathrm{~S}$ : C, 54.68; H, 6.02; N, 11.96. Found: C, 54.50; H, 6.18; $\mathrm{N}, 11.79$. Characteristic ${ }^{1} \mathrm{H}$-NMR peaks for dimer 158: ${ }^{1} \mathrm{H}$ NMR $\left(\mathrm{CDCl}_{3}, 300 \mathrm{MHz}, 52{ }^{\circ} \mathrm{C}\right) \delta 8.02(\mathrm{~d}, J=8.1 \mathrm{~Hz}, 2 \mathrm{H}, \mathrm{Ar} \mathrm{H}), 7.75$ $(\mathrm{dm}, J=8.1 \mathrm{~Hz}, 2 \mathrm{H}, \mathrm{Ar} \mathrm{H}), 7.44(\mathrm{~m}, 2 \mathrm{H}, \mathrm{Ar} \mathrm{H}), 7.31(\mathrm{~m}, 2 \mathrm{H}, \mathrm{Ar} \mathrm{H}), 1.71\left(\mathrm{~s}, 12 \mathrm{H}, \mathrm{C}_{\left.\left(\mathrm{CH}_{3}\right)_{2}\right)}\right.$.

\section{$N$-(1,1-Dimethyl-butyl)- $N^{\prime}$-(tert-butoxycarbonyl)hydrazinecarboxylic acid tert-butyl ester (160)

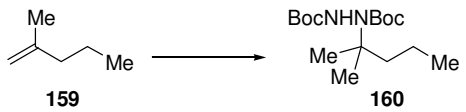

Following GP1 Hydrohydrazination product 160 (140 mg, $0.443 \mathrm{mmol}, 88 \%)$ was obtained as a colorless solid with 2methylpentene (159) $(62 \mu \mathrm{l}, 0.50 \mathrm{mmol}, 1.0$ equiv), phenylsilane ( $90 \mu \mathrm{l}, 0.75 \mathrm{mmol}, 1.5$ equiv) and di-tert-butyl azodicarboxylate (2) $(0.23 \mathrm{~g}, 1.0 \mathrm{mmol}, 2.0$ equiv) in $5 \mathrm{~h}$ with $5 \mathrm{~mol} \%$ catalyst 20 after purification by column chromatography (AcOEt/hexane 1:10). Following GP3 Hydrohydrazination product 160 (137 mg, $0.433 \mathrm{mmol}, 86 \%)$ was obtained as a colorless solid with 2methylpentene (159) $(62 \mu \mathrm{L}, 0.50 \mathrm{mmol}, 1.0$ equiv) in $2 \mathrm{~h}$ with $2 \mathrm{~mol} \%$ catalyst 23 after purification by column chromatography (AcOEt/hexane 1:10). $\mathrm{R}_{\mathrm{f}}(\mathrm{AcOEt} / \mathrm{hexane} 1: 5)$ 0.50; $\mathrm{Mp} 63-64{ }^{\circ} \mathrm{C} ;{ }^{1} \mathrm{H} \mathrm{NMR}\left(\mathrm{CDCl}_{3}, 300 \mathrm{MHz}, 52{ }^{\circ} \mathrm{C}\right) \delta 6.10(\mathrm{br} \mathrm{s}, 1 \mathrm{H}, \mathrm{NH}), 1.87$ (td, $\left.J=11.9,4.9 \mathrm{~Hz}, 1 \mathrm{H}, \mathrm{CH}_{2} \mathrm{CN}\right), 1.59\left(\mathrm{td}, J=11.9,4.9 \mathrm{~Hz}, 1 \mathrm{H}, \mathrm{CH}_{2} \mathrm{CN}\right), 1.44\left(\mathrm{~s}, 9 \mathrm{H}, \mathrm{OCCH}_{3}\right), 1.43\left(\mathrm{~s}, 9 \mathrm{H}, \mathrm{OCCH}_{3}\right), 1.39(\mathrm{~s}$, $\left.3 \mathrm{H}, \mathrm{CNCH}_{3}\right), 1.36-1.08\left(\mathrm{~m}, 2 \mathrm{H}, \mathrm{CH}_{2} \mathrm{CH}_{3}\right), 1.22\left(\mathrm{~s}, 1 \mathrm{H}, \mathrm{CNCH}_{3}\right), 0.87\left(\mathrm{t}, J=7.5 \mathrm{~Hz}, 3 \mathrm{H}, \mathrm{CH}_{2} \mathrm{CH}_{3}\right) ;{ }^{13} \mathrm{C} \mathrm{NMR}\left(\mathrm{CDCl}_{3}, 75 \mathrm{MHz}\right.$, $\left.52{ }^{\circ} \mathrm{C}\right) \delta 156.0,154.5,80.5,62.1,42.8,28.4,28.3,27.0,26.4,17.9,14.5 ; \mathrm{IR} v 3340(\mathrm{~m}), 3272(\mathrm{~m}), 3158(\mathrm{w}), 2978(\mathrm{~s}), 2933(\mathrm{~m})$, $2874(\mathrm{~m}), 1701$ (s), 1477 (m), 1456 (m), 1392 (s), 1367 (s), 1272 (s), 1252 (s), 1166 (s), 1085 (s), 1064 (m), 1047 (m), 1018 (m), $911(\mathrm{~m}), 860(\mathrm{~m}), 786(\mathrm{~m}), 764(\mathrm{~m}), 736(\mathrm{~m}), 679(\mathrm{w}), 647(\mathrm{w}), 603(\mathrm{w}), 560(\mathrm{w})$; MS (ESI) $317.3(\mathrm{M}+\mathrm{H}), 339.2(\mathrm{M}+\mathrm{Na})$; Anal. calcd for $\mathrm{C}_{16} \mathrm{H}_{32} \mathrm{~N}_{2} \mathrm{O}_{4}: \mathrm{C}, 60.73 ; \mathrm{H}, 10.19 ; \mathrm{N}, 8.85$. Found: C, 60.70; H, 10.06; N, 8.75.

$(1 R, 5 S)-N$-(2,6,6-Trimethyl-bicyclo[3.1.1]hept-2-yl)-N'-(tert-butoxycarbonyl)-hydrazinecarboxylic acid tert-butyl ester (162) 


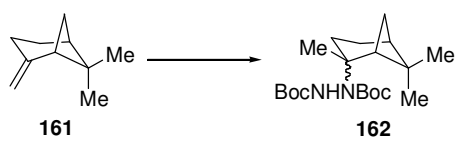

Following GP1 Hydrohydrazination product $162(155 \mathrm{mg}, 0.421 \mathrm{mmol}, 84 \%$, mixture of diastereoisomers (1:2-1:3)) was obtained as a colorless viscous oil with L- $\beta$-Pinene (161) $(78 \mu \mathrm{L}, 0.50 \mathrm{mmol}, 1.0$ equiv) in $5 \mathrm{~h}$ with 5 mol\% catalyst 20 after purification by column chromatography (AcOEt/hexane 1:15). $\mathrm{R}_{\mathrm{f}}(\mathrm{AcOEt} / \mathrm{hexane} 1: 5) 0.65 ;{ }^{1} \mathrm{H} \mathrm{NMR}\left(\mathrm{CDCl} 3,300 \mathrm{MHz}, 52{ }^{\circ} \mathrm{C}\right) \delta$ 6.03 (br s, $1 \mathrm{H}, \mathrm{NH}), 2.52-2.09\left(\mathrm{~m}, 3 \mathrm{H}, \mathrm{CH}_{2}\right.$ or $\left.\mathrm{CH}\right), 1.88-1.75\left(\mathrm{~m}, 2 \mathrm{H}, \mathrm{CH}_{2}\right.$ or $\left.\mathrm{CH}\right), 1.82\left(\mathrm{~s}, 3 \mathrm{H}, \mathrm{CNCH}_{3}\right), 1.52\left(\mathrm{~m}, 2 \mathrm{H}, \mathrm{CH}_{2}\right.$ or $\mathrm{CH}), 1.45\left(\mathrm{~s}, 9 \mathrm{H}, \mathrm{OCCH}_{3}\right), 1.45\left(\mathrm{~s}, 9 \mathrm{H}, \mathrm{OCCH}_{3}\right), 1.44\left(\mathrm{~s}, 9 \mathrm{H}, \mathrm{OCCH}_{3}\right), 1.43\left(\mathrm{~s}, 9 \mathrm{H}, \mathrm{OCCH}_{3}\right), 1.25\left(\mathrm{~s}, 3 \mathrm{H}, \mathrm{C}\left(\mathrm{CH}_{3}\right)_{2}\right), 1.13(\mathrm{~m}, 1 \mathrm{H}$,

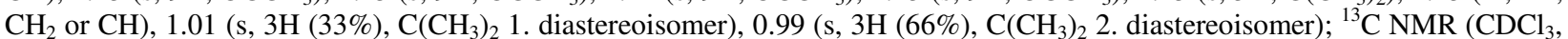
$\left.75 \mathrm{MHz}, 52{ }^{\circ} \mathrm{C}\right) \delta 155.8,154.6,80.5,68.0,67.8,52.1,51.9,40.0,39.4,38.5,37.8,30.0,29.2,28.9,28.5,28.4,28.4,27.0,26.8$, 26.5, 26.3, 23.8, 23.7; IR v 3333 (m), 3267 (m), 3157 (w), 2979 (s), 2932 (s), 2870 (m), 1707 (s), 1478 (m), 1456 (m), 1391 (s), 1367 (s), 1251 (s), 1163 (s), 1072 (m), 1049 (m), 1012 (m), 920 (m), 858 (m), 783 (m), 762 (m), 734 (m), 647 (w); HRMS (ESI) calcd for $\mathrm{C}_{20} \mathrm{H}_{36} \mathrm{~N}_{2} \mathrm{O}_{4} \mathrm{Na}^{+}(\mathrm{M}+\mathrm{Na})$ 391.2567, found 391.2555; Anal. calcd for $\mathrm{C}_{20} \mathrm{H}_{36} \mathrm{~N}_{2} \mathrm{O}_{4}$ : C, 65.19; H, 9.85; N, 7.60. Found: C, $64.98 ; \mathrm{H}, 9.98 ; \mathrm{N}, 7.50$.

$(1 S, 4 R)-N-(2,3,3-T r i m e t h y l-b i c y c l o[2.2 .1]$ hept-2-yl)-N'-(tert-butoxycarbonyl)-hydrazinecarboxylic acid tert-butyl ester (164)

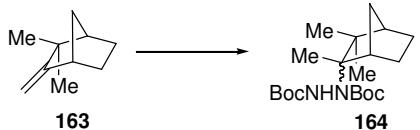

Following GP1 Hydrohydrazination product $164(124 \mathrm{mg}, 0.337 \mathrm{mmol}, 70 \%$, mixture of diastereoisomers (1:5)) was obtained as a colorless solid with D-camphene (163) $(94 \%, 70 \mathrm{mg}, 0.48 \mathrm{mmol}, 1.0$ equiv) in $5 \mathrm{~h}$ with $5 \mathrm{~mol} \%$ catalyst 20 after purification by column chromatography (AcOEt/hexane 1:15). $\mathrm{R}_{\mathrm{f}}\left(\mathrm{AcOEt} /\right.$ hexane 1:5) $0.50 ; \mathrm{Mp} \mathrm{113-114}{ }^{\circ} \mathrm{C} ;{ }^{1} \mathrm{H} \mathrm{NMR}\left(\mathrm{CDCl}_{3}, 300 \mathrm{MHz}, 52\right.$ $\left.{ }^{\circ} \mathrm{C}\right) \delta 6.08-5.91($ br s, $1 \mathrm{H}, \mathrm{NH}), 2.22\left(\mathrm{~m}, 2 \mathrm{H}, \mathrm{CH}_{2}\right.$ or $\left.\mathrm{CH}\right), 1.92-1.11\left(\mathrm{~m}, 9 \mathrm{H}, \mathrm{CH}_{2}\right.$ or $\left.\mathrm{CH}\right), 1.47\left(\mathrm{~s}, 9 \mathrm{H}, \mathrm{OCCH}_{3}\right), 1.44(\mathrm{~s}, 9 \mathrm{H}$, $\left.\mathrm{OCCH}_{3}\right), 1.05$ (s, $3 \mathrm{H}(82 \%), \mathrm{CCH}_{3}$ 1. diastereoisomer), 1.02 (s, $3 \mathrm{H}(18 \%), \mathrm{CCH}_{3} 2$. diastereoisomer), $0.97\left(\mathrm{~s}, 3 \mathrm{H}, \mathrm{CCH}_{3}\right) ;{ }^{13} \mathrm{C}$ $\mathrm{NMR}\left(\mathrm{CDCl}_{3}, 75 \mathrm{MHz}, 52{ }^{\circ} \mathrm{C}\right) \delta 156.2,155.4,80.5,72.9,72.2,51.7,50.6,48.2,47.3,36.7,35.0,28.4,28.4,27.2,26.8,24.8$, 22.6, 22.0, 19.5; IR v $3262(\mathrm{~m}), 3156(\mathrm{w}), 2978(\mathrm{~s}), 2937(\mathrm{~m}), 1704(\mathrm{~s}), 1478(\mathrm{~m}), 1455(\mathrm{~m}), 1391(\mathrm{~s}), 1367(\mathrm{~s}), 1335(\mathrm{~s}), 1253(\mathrm{~s})$, $1171(\mathrm{~s}), 1067(\mathrm{~m}), 1047(\mathrm{~m}), 1018(\mathrm{~m}), 910(\mathrm{~m}), 860(\mathrm{w}), 782(\mathrm{w}), 765(\mathrm{w}), 734(\mathrm{~m}), 648(\mathrm{w}), 618(\mathrm{w}), 569(\mathrm{w})$; MS (ESI) 369.2 $(\mathrm{M}+\mathrm{H})$; Anal. calcd for $\mathrm{C}_{20} \mathrm{H}_{36} \mathrm{~N}_{2} \mathrm{O}_{4}$ : C, 65.19; H, 9.85; N, 7.60. Found: C, 65.02; H, 9.82; N, 7.39.

\section{$N$-(1-Methyl-cyclohexyl)- $N$ '-(tert-butoxycarbonyl)hydrazinecarboxylic acid tert-butyl ester (166)}

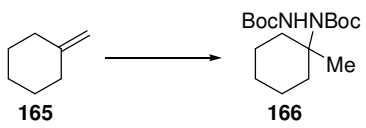

\section{Following GP1}

Hydrohydrazination product $166(147 \mathrm{mg}, 0.448 \mathrm{mmol}, 90 \%)$ was obtained as a colorless solid with methylene cyclohexane (165) $(60 \mu \mathrm{L}, 0.50 \mathrm{mmol}, 1.0$ equiv) in $5 \mathrm{~h}$ with $5 \mathrm{~mol} \%$ catalyst 20 after purification by column chromatography (AcOEt/hexane 1:15). $\mathrm{R}_{\mathrm{f}}$ (AcOEt/hexane 1:5) 0.54; $\mathrm{Mp} \mathrm{101-102}{ }^{\circ} \mathrm{C} ;{ }^{1} \mathrm{H} \mathrm{NMR}\left(\mathrm{CDCl}_{3}, 300 \mathrm{MHz}, 52{ }^{\circ} \mathrm{C}\right) \delta 6.11(\mathrm{br} \mathrm{s}, 1 \mathrm{H}, \mathrm{NH}), 2.17(\mathrm{~m}, 1 \mathrm{H}$, $\left.\mathrm{CH}_{2}\right), 1.98\left(\mathrm{~m}, 1 \mathrm{H}, \mathrm{CH}_{2}\right), 1.68-1.37\left(\mathrm{~m}, 8 \mathrm{H}, \mathrm{CH}_{2}\right), 1.47\left(\mathrm{~s}, 9 \mathrm{H}, \mathrm{OCCH}_{3}\right), 1.45\left(\mathrm{~s}, 9 \mathrm{H}, \mathrm{OCCH}_{3}\right), 1.37\left(\mathrm{~s}, 3 \mathrm{H}, \mathrm{CNCH}_{3}\right) ;{ }^{13} \mathrm{C} \mathrm{NMR}^{\mathrm{N}}$ $\left(\mathrm{CDCl}_{3}, 75 \mathrm{MHz}, 52{ }^{\circ} \mathrm{C}\right) \delta 156.3,80.5,62.1,36.8,36.2,28.4,28.3,25.8,23.9,23.2,22.7 ;$ IR $v 3344(\mathrm{w}), 2978(\mathrm{~m}), 2932(\mathrm{~m})$, 2863 (w), 1705 (s), 1479 (m), 1454 (m), 1391 (m), 1366 (s), 1341 (s), 1252 (s), 1163 (s), 1083 (m), 1069 (m), 1048 (w), 1018 (w), $962(w), 908(w), 857(w), 783(w), 762(w), 734(w)$; MS (ESI) $329.4(\mathrm{M}+\mathrm{H}), 351.3(\mathrm{M}+\mathrm{Na})$; Anal. calcd for $\mathrm{C}_{17} \mathrm{H}_{32} \mathrm{~N}_{2} \mathrm{O}_{4}$ : C, $62.17 ; \mathrm{H}, 9.82 ; \mathrm{N}, 8.53$. Found: C, 62.29; H, 9.54; N, 8.50.

2-(N, N'-Bis-(tert-butoxycarbonyl-hydrazino))-butyric acid ethyl ester (168)

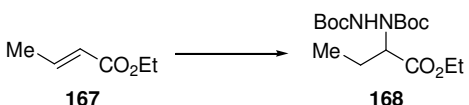

Following GP1 Hydrohydrazination product $168(113 \mathrm{mg}, 0.327 \mathrm{mmol}, 66 \%)$ was obtained as a colorless viscous oil with trans-ethyl crotonate (167) $(62 \mu \mathrm{L}, 0.50 \mathrm{mmol}, 1.0$ equiv), phenylsilane ( $90 \mu \mathrm{L}, 0.75 \mathrm{mmol}, 1.5$ equiv) and di-tert-butyl azodicarboxylate (2) $(0.23 \mathrm{~g}, 1.0 \mathrm{mmol}, 2.0$ equiv) in $12 \mathrm{~h}$ with $5 \mathrm{~mol} \%$ catalyst 20 after purification by column chromatography (AcOEt/hexane 1:10). Following GP3 Hydrohydrazination product 168 (154 mg, $0.445 \mathrm{mmol}, 88 \%)$ was obtained as a colorless viscous oil with trans-ethyl crotonate (167) $(62 \mu \mathrm{L}, 0.50 \mathrm{mmol}, 1.0$ equiv) in $6 \mathrm{~h}$ with 2 mol\% catalyst 23 after purification by column chromatography (AcOEt/hexane 1:10). $\mathrm{R}_{\mathrm{f}}(\mathrm{AcOEt} /$ hexane $1: 5) 0.30 ;{ }^{1} \mathrm{H} \mathrm{NMR}\left(\mathrm{CDCl}_{3}, 300 \mathrm{MHz}, 52{ }^{\circ} \mathrm{C}\right) \delta 6.32(\mathrm{br} \mathrm{s}, 1 \mathrm{H}$, $\mathrm{NH}), 4.61($ br s, $1 \mathrm{H}, \mathrm{CHN}), 4.23-4.10\left(\mathrm{~m}, 2 \mathrm{H}, \mathrm{OCH}_{2}\right), 1.97-1.75\left(\mathrm{~m}, 1 \mathrm{H}, \mathrm{CH}_{2} \mathrm{CHN}\right), 1.48\left(\mathrm{~s}, 9 \mathrm{H}, \mathrm{OCCH}_{3}\right), 1.48(\mathrm{~s}, 9 \mathrm{H}$, $\left.\mathrm{OCCH}_{3}\right), 1.28\left(\mathrm{t}, J=7.2 \mathrm{~Hz}, 3 \mathrm{H}, \mathrm{OCH}_{2} \mathrm{CH}_{3}\right), 1.10\left(\mathrm{t}, J=7.5 \mathrm{~Hz}, 3 \mathrm{H}, \mathrm{CHNCH}_{2} \mathrm{CH}_{3}\right) ;{ }^{13} \mathrm{C} \mathrm{NMR}\left(\mathrm{CDCl}_{3}, 75 \mathrm{MHz}, 52{ }^{\circ} \mathrm{C}\right) \delta 171.7$, 155.5, 81.7, 80.8, 61.1, 28.3, 28.2, 22.4, 14.2, 11.2; IR v 3326 (w), 2980 (m), 2936 (m), 1738 (s), 1714 (s), 1479 (m), 1393 (m), $1368(\mathrm{~s}), 1330(\mathrm{~m}), 1299(\mathrm{~m}), 1237(\mathrm{~m}), 1155(\mathrm{~s}), 1087(\mathrm{~m}), 1021(\mathrm{~m}), 940(\mathrm{w}), 856(\mathrm{w}), 782(\mathrm{w}), 700$ (w); HRMS (ESI) calcd for $\mathrm{C}_{16} \mathrm{H}_{30} \mathrm{~N}_{2} \mathrm{O}_{6} \mathrm{Na}^{+}$(M+Na) 369.1996, found 369.2000; Anal. calcd for $\mathrm{C}_{16} \mathrm{H}_{30} \mathrm{~N}_{2} \mathrm{O}_{6}: \mathrm{C}, 55.47 ; \mathrm{H}, 8.73 ; \mathrm{N}$, 8.09. Found: C, 55.68; H, 8.53; N, 8.00. 
$N$-(3-Hydroxy-1,1-dimethyl-propyl)- $N$ '-(tert-butoxycarbonyl)hydrazinecarboxylic acid tert-butyl ester (170)

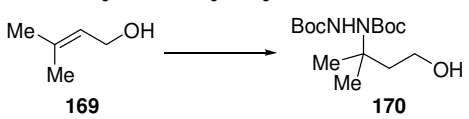

Following GP1 Hydrohydrazination product 170 (111 mg, $0.350 \mathrm{mmol}, 70 \%)$ was obtained as a colorless solid with 2-methyl2-buten-4-ol (169) $(51 \mu \mathrm{L}, 0.50 \mathrm{mmol}, 1.0$ equiv) in $8 \mathrm{~h}$ with $5 \mathrm{~mol} \%$ catalyst 20 after purification by column chromatography (AcOEt/hexane 1:7-1:2). $\mathrm{R}_{\mathrm{f}}$ (AcOEt/hexane 1:2) 0.18; Mp 100-101 ${ }^{\circ} \mathrm{C} ;{ }^{1} \mathrm{H} \mathrm{NMR}\left(\mathrm{CDCl}_{3}, 300 \mathrm{MHz}, 52{ }^{\circ} \mathrm{C}\right) \delta 6.37(\mathrm{br} \mathrm{s}, 1 \mathrm{H}, \mathrm{NH})$, 3.77-3.71 (m, $\left.2 \mathrm{H}, \mathrm{CH}_{2} \mathrm{OH}\right), 3.18$ (br s, $\left.1 \mathrm{H}, \mathrm{OH}\right), 2.22$ (br s, $\left.1 \mathrm{H}, \mathrm{CH}_{2} \mathrm{CN}\right), 1.86$ (br s, $\left.1 \mathrm{H}, \mathrm{CH}_{2} \mathrm{CN}\right), 1.45\left(\mathrm{~s}, 9 \mathrm{H}, \mathrm{OCCH}_{3}\right), 1.44(\mathrm{~s}$,

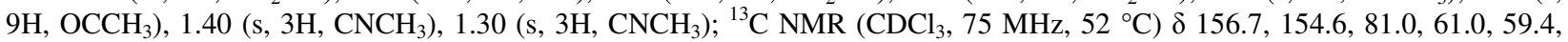
42.2, 28.4, 28.3, 27.9, 27.5; IR v $3447(\mathrm{~m}, \mathrm{sh}), 3329(\mathrm{~m}), 2980(\mathrm{~s}), 2933(\mathrm{~m}), 1713(\mathrm{~s}), 1479(\mathrm{~m}), 1456(\mathrm{~m}), 1392(\mathrm{~s}), 1368(\mathrm{~s})$, 1255 (s), 1161 (s), 1081 (s), 1050 (m), 1023 (m), 976 (w), 911 (m), 858 (w), 785 (w), 763 (m), 734 (m), 647 (w), 609 (w); MS (ESI) $341.3(\mathrm{M}+\mathrm{Na})$; Anal. calcd for $\mathrm{C}_{15} \mathrm{H}_{30} \mathrm{~N}_{2} \mathrm{O}_{5}$ : C, 56.58; H, 9.50; N, 8.80. Found: C, 56.51; H, 9.54; N, 8.83.

$N$-(Bicyclo[2.2.1]hept-2-yl)- $N$ '-(tert-butoxycarbonyl)hydrazinecarboxylic acid tert-butyl ester (172)

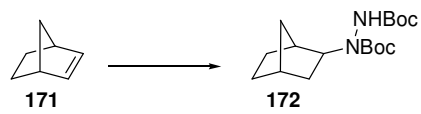

Following GP1 Hydrohydrazination product $172(103 \mathrm{mg}, 0.316 \mathrm{mmol}, 66 \%)$ was obtained as a colorless solid with norbornene (171) (45 mg, $0.48 \mathrm{mmol}, 1.0$ equiv) in $7 \mathrm{~h}$ with $5 \mathrm{~mol} \%$ catalyst 20 after purification by column chromatography (AcOEt/hexane 1:10). Following GP3 Hydrohydrazination product 172 (179 $\mathrm{mg}, 0.548 \mathrm{mmol}, 98 \%)$ was obtained as a colorless solid with norbornene (171) $(53 \mathrm{mg}, 0.56 \mathrm{mmol}, 1.0$ equiv) in $7 \mathrm{~h}$ with $2 \mathrm{~mol} \%$ catalyst 23 after purification by column chromatography (AcOEt/hexane 1:10). $\mathrm{R}_{\mathrm{f}}$ (AcOEt/hexane 1:5) 0.40; Mp 169-170 ${ }^{\circ} \mathrm{C} ;{ }^{1} \mathrm{H} \mathrm{NMR}\left(\mathrm{CDCl}_{3}, 300 \mathrm{MHz}, 52{ }^{\circ} \mathrm{C}\right) \delta 6.06(\mathrm{br}, 1 \mathrm{H}, \mathrm{NH})$, $3.92(\mathrm{~m}, 1 \mathrm{H}, \mathrm{CHN}), 2.35-2.23(\mathrm{~m}, 2 \mathrm{H}, \mathrm{CH}), 1.68-1.08\left(\mathrm{~m}, 8 \mathrm{H}, \mathrm{CH}_{2}\right), 1.47\left(\mathrm{~s}, 9 \mathrm{H}, \mathrm{OCCH}_{3}\right), 1.46\left(\mathrm{~s}, 9 \mathrm{H}, \mathrm{OCCH}_{3}\right) ;{ }^{13} \mathrm{C} \mathrm{NMR}$ $\left(\mathrm{CDCl}_{3}, 75 \mathrm{MHz}, 52{ }^{\circ} \mathrm{C}\right) \delta 155.7,155.1,80.8,60.6,41.2,37.2,36.2,35.8,28.4,28.3,27.9$; IR $v 3318(\mathrm{w}), 2958(\mathrm{~m}), 2874(\mathrm{~m})$, $1701(\mathrm{~s}), 1516(\mathrm{~m}), 1479(\mathrm{~m}), 1454(\mathrm{w}), 1410(\mathrm{~m}), 1392(\mathrm{~m}), 1366$ (s), $1348(\mathrm{~s}), 1286(\mathrm{~s}), 1253(\mathrm{~s}), 1163$ (s), $1130(\mathrm{~m}), 1111(\mathrm{~m})$, 1067 (m), 966 (m), 918 (w), 898 (w), 859 (w), $834(\mathrm{w}), 784(\mathrm{w}), 760(\mathrm{w}), 735(\mathrm{w}), 600(\mathrm{w})$; MS (ESI) 327.2 (M+H), 349.3 $(\mathrm{M}+\mathrm{Na})$; Anal. calcd for $\mathrm{C}_{17} \mathrm{H}_{30} \mathrm{~N}_{2} \mathrm{O}_{4}$ : C, 62.55; H, 9.26; N, 8.58. Found: C, 62.70; H, 8.98; N, 8.50.

$N$-(Cyclooctyl)- $N$ '-(tert-butoxycarbonyl)hydrazinecarboxylic acid tert-butyl ester (174)

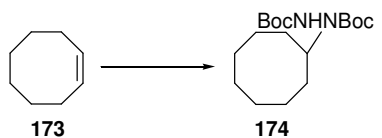

Following GP1 Hydrohydrazination product $174(104 \mathrm{mg}, 0.304 \mathrm{mmol}, 62 \%)$ was obtained as a colorless solid with cyclooctene (173) (freshly distilled, $65 \mu \mathrm{L}, 0.49 \mathrm{mmol}, 1.0$ equiv), phenylsilane (95 $\mu \mathrm{L}, 0.75 \mathrm{mmol}, 1.5$ equiv) and di-tert-butyl azodicarboxylate (2) $(0.23 \mathrm{~g}, 1.0 \mathrm{mmol}, 2.0$ equiv) in $24 \mathrm{~h}$ with $5 \mathrm{~mol} \%$ catalyst 20 after purification by column chromatography (AcOEt/hexane 1:10). Following GP3 Hydrohydrazination product 174 (163 mg, $0.476 \mathrm{mmol}$, 95\%) was obtained as a colorless solid with cyclooctene (173) (freshly distilled, $65 \mu \mathrm{L}, 0.49 \mathrm{mmol}, 1.0$ equiv) in $2.5 \mathrm{~h}$ with $2 \mathrm{~mol} \%$ catalyst 23 after purification by column chromatography (AcOEt/hexane 1:10).

$N$-(Cyclopentyl)- $N$ '-(tert-butoxycarbonyl)hydrazinecarboxylic acid tert-butyl ester (176)

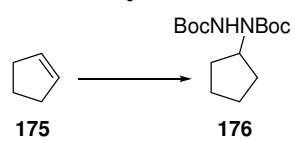

Following GP1 Hydrohydrazination product $176(111 \mathrm{mg}, 0.370 \mathrm{mmol}, 74 \%)$ was obtained as a colorless solid with cyclopentene (175) (44 $\mu \mathrm{L}, 0.50 \mathrm{mmol}, 1.0$ equiv), phenylsilane ( $95 \mu \mathrm{L}, 0.75 \mathrm{mmol}, 1.5$ equiv) and di-tert-butyl azodicarboxylate (2) $(0.23 \mathrm{~g}, 1.0 \mathrm{mmol}, 2.0$ equiv) in $8 \mathrm{~h}$ with $5 \mathrm{~mol} \%$ catalyst 20 after purification by column chromatography (AcOEt/hexane 1:10). Following GP3 Hydrohydrazination product 176 (141 mg, $0.469 \mathrm{mmol}, 94 \%)$ was obtained as a colorless solid with cyclopentene (175) (44 $\mu \mathrm{L}, 0.50 \mathrm{mmol}, 1.0$ equiv) in $2 \mathrm{~h}$ with $2 \mathrm{~mol} \%$ catalyst 23 after purification by column chromatography (AcOEt/hexane 1:10). $\mathrm{R}_{\mathrm{f}}$ (AcOEt/hexane 1:5) 0.40; Mp 154-155 ${ }^{\circ} \mathrm{C} ;{ }^{1} \mathrm{H} \mathrm{NMR}\left(\mathrm{CDCl}_{3}, 300 \mathrm{MHz}, 52{ }^{\circ} \mathrm{C}\right) \delta 6.00(\mathrm{br}, 1 \mathrm{H}, \mathrm{NH})$, $4.46(\mathrm{~m}, 1 \mathrm{H}, \mathrm{CHN}), 1.83-1.26\left(\mathrm{~m}, 8 \mathrm{H}, \mathrm{CH}_{2}\right), 1.48\left(\mathrm{~s}, 9 \mathrm{H}, \mathrm{OCCH}_{3}\right), 1.47\left(\mathrm{~s}, 9 \mathrm{H}, \mathrm{OCCH}_{3}\right) ;{ }^{13} \mathrm{C} \mathrm{NMR}\left(\mathrm{CDCl}_{3}, 75 \mathrm{MHz}, 52{ }^{\circ} \mathrm{C}\right) \delta$ 155.9, 154.9, 80.8, 59.0, 29.1, 28.4, 28.3, 23.8; IR v 3312 (w), $2976(\mathrm{~m}), 2869(\mathrm{~m}), 1700$ (s), $1517(\mathrm{~m}), 1480(\mathrm{w}), 1453(\mathrm{w}), 1405$ (s), $1366(\mathrm{~m}), 1345(\mathrm{~m}), 1292(\mathrm{~m}), 1250(\mathrm{~m}), 1157(\mathrm{~s}), 1126(\mathrm{~m}), 1054(\mathrm{w}), 1028(\mathrm{w}), 948(\mathrm{w}), 894(\mathrm{w}), 858(\mathrm{w}), 758(\mathrm{w}), 736$ (w), $607(w)$; MS (ESI) $301.2(\mathrm{M}+\mathrm{H}), 323.2(\mathrm{M}+\mathrm{Na}), 623.2(2 \mathrm{M}+\mathrm{Na})$; Anal. calcd for $\mathrm{C}_{15} \mathrm{H}_{28} \mathrm{~N}_{2} \mathrm{O}_{4}$ : C, 59.98; H, 9.39; N, 9.33. Found: C, 60.07; H, 9.39; N, 9.15.

$N$-(3-Hydroxy-1-methyl-propyl)- $N$ '-(tert-butoxycarbonyl)hydrazinecarboxylic acid tert-butyl ester (178)

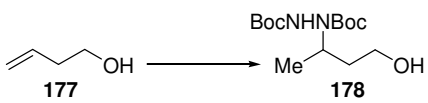

Following GP1 Hydrohydrazination product 178 (34 mg, $0.11 \mathrm{mmol}, 22 \%)$ was obtained as a colorless viscous oil with but-1ene-4-ol (177) (43 $\mu \mathrm{L}, 0.50 \mathrm{mmol}, 1.0$ equiv) in $12 \mathrm{~h}$ with 5 mol\% catalyst 20 after purification by column chromatography 
(AcOEt/hexane 1:2). Following GP3 Hydrohydrazination product 178 (111 mg, $0.36 \mathrm{mmol}, 72 \%)$ was obtained as a colorless viscous oil with but-1-ene-4-ol (177) $(43 \mu \mathrm{L}, 0.50 \mathrm{mmol}, 1.0$ equiv) in $2.5 \mathrm{~h}$ with $2 \mathrm{~mol} \%$ catalyst 23 after purification by column chromatography (AcOEt/hexane 1:2). Following GP3, but with PMHS Hydrohydrazination product 178 (90 mg, 0.30 mmol, $60 \%)$ was obtained as colorless viscous oil with but-1-ene-4-ol (177) $(43 \mu \mathrm{L}, 0.50 \mathrm{mmol}, 1.0$ equiv) and PMHS $(0.10 \mathrm{~mL}, 1.6 \mathrm{H}$ equiv) in $10 \mathrm{~h}$ at $23{ }^{\circ} \mathrm{C}$ with $2 \mathrm{~mol} \%$ catalyst 23 after purification by column chromatography (AcOEt/hexane $\left.1: 2\right)$. $\mathrm{R}_{\mathrm{f}}$ (AcOEt/hexane 1:2) 0.20; ${ }^{1} \mathrm{H} \mathrm{NMR}\left(\mathrm{CDCl}_{3}, 300 \mathrm{MHz}, 52{ }^{\circ} \mathrm{C}\right) \delta 6.11$ (br s, $\left.1 \mathrm{H}, \mathrm{NH}\right), 4.38$ (br s, $\left.1 \mathrm{H}, \mathrm{CHN}\right), 3.73-3.63(\mathrm{~m}, 2 \mathrm{H}$, $\left.\mathrm{CH}_{2} \mathrm{OH}\right), 2.77($ br s, $1 \mathrm{H}, \mathrm{OH}), 1.68-1.51\left(\mathrm{~m}, 2 \mathrm{H}, \mathrm{CH}_{2} \mathrm{CN}\right), 1.47\left(\mathrm{~s}, 9 \mathrm{H}, \mathrm{OCCH}_{3}\right), 1.47\left(\mathrm{~s}, 9 \mathrm{H}, \mathrm{OCCH}_{3}\right), 1.15(\mathrm{~d}, J=6.9 \mathrm{~Hz}, 3 \mathrm{H}$, $\left.\mathrm{CNCH}_{3}\right) ;{ }^{13} \mathrm{C} \mathrm{NMR}\left(\mathrm{CDCl}_{3}, 75 \mathrm{MHz}, 52{ }^{\circ} \mathrm{C}\right) \delta 155.9,155.6,81.4,81.1,59.7,50.0,37.2,28.3,28.3,18.3$; IR v 3449 (w, sh), 3312 (m), 2979 (m), 2935 (m), 1705 (s), 1479 (m), 1456 (m), 1394 (s), 1368 (s), 1251 (s), 1162 (s), 1080 (m), 1047 (m), 1017 (m), 914 (w), $854(\mathrm{w}), 761(\mathrm{w}), 735(\mathrm{~m}), 647(\mathrm{w}), 577(\mathrm{w})$; HRMS(ESI) calcd for $\mathrm{C}_{14} \mathrm{H}_{28} \mathrm{~N}_{2} \mathrm{O}_{5} \mathrm{Na}^{+}(\mathrm{M}+\mathrm{Na}) 327.1890$, found 327.1888; Anal. calcd for $\mathrm{C}_{14} \mathrm{H}_{28} \mathrm{~N}_{2} \mathrm{O}_{5}$ : C, 55.24; H, 9.27; N, 9.20. Found: C, 55.40; H, 9.51; N, 8.93.

$N$-(3-Hydroxy-1-methyl-propyl)- $N$ '-(tert-butoxycarbonyl)hydrazinecarboxylic acid tert-butyl ester (178) and $N$-(2Hydroxy-1-ethyl-ethyl)- $N$ '-(tert-butoxycarbonyl)hydrazine carboxylic acid tert-butyl ester (180)

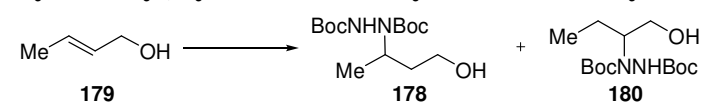

Following GP1 Hydrohydrazination product $178(67 \mathrm{mg}, 0.22 \mathrm{mmol}, 44 \%)$ was obtained as a colorless viscous oil together with the regioisomeric product $180(15 \mathrm{mg}, 0.05 \mathrm{mmol}, 10 \%)$ with crotyl alcohol (179) (43 $\mu \mathrm{L}, 0.50 \mathrm{mmol}, 1.0$ equiv), phenylsilane $(0.12 \mathrm{~mL}, 1.0 \mathrm{mmol}, 2.0$ equiv) and di-tert-butyl azodicarboxylate (2) $(0.34 \mathrm{~g}, 1.5 \mathrm{mmol}, 3.0$ equiv) in $24 \mathrm{~h}$ with 5 mol\% catalyst 20 after purification by column chromatography (AcOEt/hexane 1:3). Following GP3 Hydrohydrazination product $178(88 \mathrm{mg}, 0.29 \mathrm{mmol}, 58 \%)$ was obtained as a colorless viscous oil together with the regioisomeric product 180 (49 $\mathrm{mg}, 0.16$ mmol, $32 \%$, colorless solid) with crotyl alcohol (179) $(43 \mu \mathrm{L}, 0.50 \mathrm{mmol}, 1.0$ equiv) in $2 \mathrm{~h}$ with 2 mol\% catalyst after purification by column chromatography (AcOEt/hexane 1:6-1:1).

\section{$\boldsymbol{N}$-(2-Hydroxy-1-ethyl-ethyl)- $\boldsymbol{N}^{\prime}$-(tert-butoxycarbonyl)hydrazine carboxylic acid tert-butyl ester (180)}

$\mathrm{R}_{\mathrm{f}}$ (AcOEt/hexane 1:2) 0.33; Mp 144-145 ${ }^{\circ} \mathrm{C} ;{ }^{1} \mathrm{H} \mathrm{NMR}\left(\mathrm{CDCl}_{3}, 300 \mathrm{MHz}, 52{ }^{\circ} \mathrm{C}\right) \delta 6.17$ (br s, $\left.1 \mathrm{H}, \mathrm{NH}\right), 4.12(\mathrm{br} \mathrm{s}, 1 \mathrm{H}, \mathrm{CHN})$, 3.42-3.39 (m, 2H, $\left.\mathrm{CH}_{2} \mathrm{OH}\right), 1.48\left(\mathrm{~s}, 9 \mathrm{H}, \mathrm{OCCH}_{3}\right), 1.46\left(\mathrm{~s}, 9 \mathrm{H}, \mathrm{OCCH}_{3}\right), 1.40-1.23\left(\mathrm{~m}, 2 \mathrm{H}, \mathrm{CH}_{2} \mathrm{CH}_{3}\right), 0.86(\mathrm{t}, J=7.5 \mathrm{~Hz}, 3 \mathrm{H}$, $\left.\mathrm{CH}_{2} \mathrm{CH}_{3}\right) ;{ }^{13} \mathrm{C}$ NMR $\left(\mathrm{CDCl}_{3}, 75 \mathrm{MHz}, 25{ }^{\circ} \mathrm{C}\right) \delta 158.3,157.7,156.1,155.2,82.3,82.1,81.8,81.2,62.2,61.9,59.8,28.2,28.1$, 22.0, 21.2, 10.6; IR v 3363 (w), 3206 (w), 2973 (m), 1712 (s), 1538 (w), 1456 (w), 1394 (m), 1367 (m), 1342 (m), 1289 (m), 1256

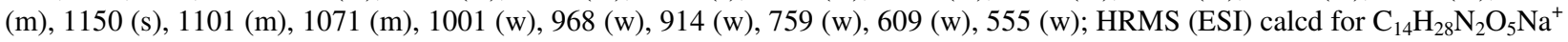
$(\mathrm{M}+\mathrm{Na})$ 327.1890, found 327.1885; Anal. calcd for $\mathrm{C}_{14} \mathrm{H}_{28} \mathrm{~N}_{2} \mathrm{O}_{5}$ : C, 55.24; H, 9.27; N, 9.20. Found: C, 55.32; H, 9.18; N, 9.22.

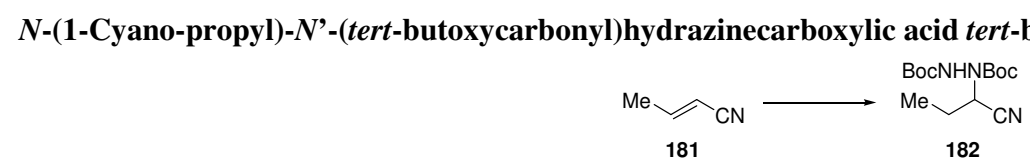

Following GP1 Hydrohydrazination product $182(69 \mathrm{mg}, 0.23 \mathrm{mmol}, 46 \%)$ was obtained as a colorless solid with crotyl nitrile (181) $(41 \mu \mathrm{L}, 0.50 \mathrm{mmol}, 1.0$ equiv) in $18 \mathrm{~h}$ with $5 \mathrm{~mol} \%$ catalyst 20 after purification by column chromatography (AcOEt/hexane 1:10). Following GP3 Hydrohydrazination product 182 (67 mg, 0.22 mmol, 44\%) was obtained as a colorless solid with crotyl nitrile (181) $(41 \mu \mathrm{L}, 0.50 \mathrm{mmol}, 1.0$ equiv) in $2.5 \mathrm{~h}$ with $2 \mathrm{~mol} \%$ catalyst 23 after purification by column chromatography (AcOEt/hexane 1:10). $\mathrm{R}_{\mathrm{f}}$ (AcOEt/hexane 1:5) 0.30; $\mathrm{Mp} 105-107{ }^{\circ} \mathrm{C} ;{ }^{1} \mathrm{H} \mathrm{NMR}\left(\mathrm{CDCl}_{3}, 300 \mathrm{MHz}, 52{ }^{\circ} \mathrm{C}\right) \delta 6.31$ (br s, $1 \mathrm{H}, \mathrm{NH}), 4.91$ (br s, $1 \mathrm{H}, \mathrm{CHN}), 1.95-1.85\left(\mathrm{~m}, 2 \mathrm{H}, \mathrm{CH}_{2}\right), 1.49\left(\mathrm{~s}, 9 \mathrm{H}, \mathrm{OCCH}_{3}\right), 1.48\left(\mathrm{~s}, 9 \mathrm{H}, \mathrm{OCCH}_{3}\right), 1.07(\mathrm{t}, J=7.5 \mathrm{~Hz}$, $\left.3 \mathrm{H}, \mathrm{CH}_{2} \mathrm{CH}_{3}\right) ;{ }^{13} \mathrm{C} \mathrm{NMR}\left(\mathrm{CDCl}_{3}, 75 \mathrm{MHz}, 52{ }^{\circ} \mathrm{C}\right) \delta 155.0,153.5,117.0,83.1,82.0,51.9,28.2,25.0,10.3 ; \mathrm{IR} v 3316(\mathrm{~m}), 2980$ (s), 2938 (s), 2883 (w), 2254 (w), 2176 (w), 1714 (s), 1479 (m), 1459 (m), 1393 (s), 1370 (s), 1299 (s), 1254 (s), 1151 (s), 1110 $(\mathrm{m}), 1093(\mathrm{~m}), 1051(\mathrm{~m}), 1017(\mathrm{~m}), 936(\mathrm{~m}), 891(\mathrm{w}), 848(\mathrm{~m}), 785(\mathrm{w}), 761(\mathrm{~m}), 737(\mathrm{w}), 597$ (w); HRMS (ESI) calcd for $\mathrm{C}_{14} \mathrm{H}_{25} \mathrm{~N}_{3} \mathrm{O}_{4} \mathrm{Na}^{+}\left(\mathrm{M}+\mathrm{Na}\right.$ ) 322.1737, found 322.1735; Anal. calcd for $\mathrm{C}_{14} \mathrm{H}_{25} \mathrm{~N}_{3} \mathrm{O}_{4}$ : C, 56.17; H, 8.42; N, 14.04. Found: C, 56.02; $\mathrm{H}, 8.40 ; \mathrm{N}, 13.97$.

$N$-(1-ethyl-propyl)-N'-(tert-butoxycarbonyl)hydrazinecarboxylic acid tert-butyl ester (184)

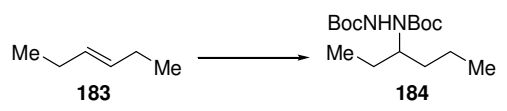

Following GP1 Hydrohydrazination product $184(25 \mathrm{mg}, 0.079 \mathrm{mmol}, 16 \%)$ was obtained as a colorless solid with trans-3hexene (182) $(62 \mu \mathrm{L}, 0.50 \mathrm{mmol}, 1.0$ equiv) in $10 \mathrm{~h}$ with $5 \mathrm{~mol} \%$ catalyst 20 after purification by column chromatography (AcOEt/hexane 1:15). Following GP3 Hydrohydrazination product 184 (105 mg, $0.332 \mathrm{mmol}$, 66\%) was obtained as a colorless solid with trans-3-hexene (182) $(62 \mu \mathrm{L}, 0.50 \mathrm{mmol}, 1.0$ equiv) in $3 \mathrm{~h}$ with $2 \mathrm{~mol} \%$ catalyst 23 after purification by column chromatography (AcOEt/hexane 1:15). $\mathrm{R}_{\mathrm{f}}$ (AcOEt/hexane 1:5) 0.50; Mp 80-82 ${ }^{\circ} \mathrm{C} ;{ }^{1} \mathrm{H}$ NMR $\left(\mathrm{CDCl}_{3}, 300 \mathrm{MHz}, 52{ }^{\circ} \mathrm{C}\right) \delta 5.87(\mathrm{br}$ $\mathrm{s}, 1 \mathrm{H}, \mathrm{NH}), 3.94($ br s, $1 \mathrm{H}, \mathrm{CHN}), 1.57-1.23\left(\mathrm{~m}, 6 \mathrm{H}, \mathrm{CH}_{2}\right), 1.46\left(\mathrm{~s}, 9 \mathrm{H}, \mathrm{OCCH}_{3}\right), 1.45\left(\mathrm{~s}, 9 \mathrm{H}, \mathrm{OCCH}_{3}\right), 0.89(\mathrm{t}, J=7.2 \mathrm{~Hz}, 6 \mathrm{H}$, $\left.\mathrm{CH}_{2} \mathrm{CH}_{3}\right) ;{ }^{13} \mathrm{C} \mathrm{NMR}\left(\mathrm{CDCl}_{3}, 75 \mathrm{MHz}, 52{ }^{\circ} \mathrm{C}\right) \delta 155.5,80.7,58.6,34.5,28.3,28.3,25.6,19.8,14.0,11.2 ; \mathrm{IR} v 3362(\mathrm{~m}), 3160$ (w), $2968(\mathrm{~s}), 2934$ (s), $2875(\mathrm{~m}), 1750$ (s), 1705 (s), 1479 (m), 1456 (m), 1393 (s), 1367 (s), 1338 (s), 1303 (m), 1254 (s), 1156 (s), $1106(\mathrm{~s}), 1046(\mathrm{~m}), 1016(\mathrm{~m}), 935(\mathrm{~m}), 858(\mathrm{w}), 797(\mathrm{w}), 760(\mathrm{~m}), 617(\mathrm{w}) ; \mathrm{MS}(\mathrm{ESI}) 317.1(\mathrm{M}+\mathrm{H}), 339.1(\mathrm{M}+\mathrm{Na})$; Anal. calcd for $\mathrm{C}_{16} \mathrm{H}_{32} \mathrm{~N}_{2} \mathrm{O}_{4}: \mathrm{C}, 60.73 ; \mathrm{H}, 10.19 ; \mathrm{N}, 8.85$. Found: $\mathrm{C}, 60.50 ; \mathrm{H}, 10.43 ; \mathrm{N}, 8.78$. 


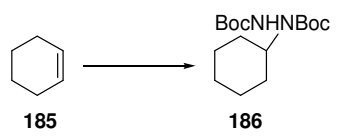

Following GP1 Hydrohydrazination product 186 (39 $\mathrm{mg}, 0.12 \mathrm{mmol}, 24 \%$ ) was obtained as a colorless solid with cyclohexene (185) $(51 \mu \mathrm{L}, 0.50 \mathrm{mmol}, 1.0$ equiv), phenylsilane ( $95 \mu \mathrm{L}, 0.75 \mathrm{mmol}, 1.5$ equiv) and di-tert-butyl azodicarboxylate $(2)(0.23 \mathrm{~g}$, $1.0 \mathrm{mmol}, 2.0$ equiv) in $24 \mathrm{~h}$ with $5 \mathrm{~mol} \%$ catalyst 20 after purification by column chromatography (AcOEt/hexane $1: 10$ ). Following GP3 Hydrohydrazination product 186 (142 mg, $0.452 \mathrm{mmol}, 90 \%)$ was obtained as a colorless solid with cyclohexene (185) $(51 \mu \mathrm{L}, 0.50 \mathrm{mmol}, 1.0$ equiv) in $2 \mathrm{~h}$ with $2 \mathrm{~mol} \%$ catalyst 23 after purification by column chromatography (AcOEt/hexane 1:10). $\mathrm{R}_{\mathrm{f}}$ (AcOEt/hexane 1:5) 0.42; Mp 147-148 ${ }^{\circ} \mathrm{C} ;{ }^{1} \mathrm{H}$ NMR $\left(\mathrm{CDCl}_{3}, 300 \mathrm{MHz}, 52{ }^{\circ} \mathrm{C}\right) \delta 5.98(\mathrm{br} \mathrm{s}, 1 \mathrm{H}, \mathrm{NH}), 3.93(\mathrm{br} \mathrm{s}, 1 \mathrm{H}$, $\mathrm{CHN}), 1.80-1.06\left(\mathrm{~m}, 10 \mathrm{H}, \mathrm{CH}_{2}\right), 1.48\left(\mathrm{~s}, 9 \mathrm{H}, \mathrm{OCCH}_{3}\right), 1.47\left(\mathrm{~s}, 9 \mathrm{H}, \mathrm{OCCH}_{3}\right) ;{ }^{13} \mathrm{C} \mathrm{NMR}\left(\mathrm{CDCl}_{3}, 75 \mathrm{MHz}, 52{ }^{\circ} \mathrm{C}\right) \delta 155.8,154.6$, 80.8, 56.9, 30.3, 28.4, 28.3, 25.7, 25.6; IR v $3314(\mathrm{~m}), 2976(\mathrm{~m}), 2931(\mathrm{~s}), 2857(\mathrm{~m}), 1699(\mathrm{~s}), 1519(\mathrm{~s}), 1453(\mathrm{~m}), 1398(\mathrm{~s}), 1365$ (s), $1318(\mathrm{~s}), 1290(\mathrm{~s}), 1268(\mathrm{~s}), 1256(\mathrm{~s}), 1236(\mathrm{~s}), 1172(\mathrm{~s}), 1152(\mathrm{~s}), 1116(\mathrm{~m}), 1060(\mathrm{~m}), 1026(\mathrm{w}), 1003(\mathrm{~m}), 932(\mathrm{w}), 904(\mathrm{~m})$, $869(\mathrm{~m}), 858(\mathrm{~m}), 784(\mathrm{w}), 758(\mathrm{~m}), 611(\mathrm{w})$; MS (ESI) $315.3(\mathrm{M}+\mathrm{H}), 337.3(\mathrm{M}+\mathrm{Na}), 651.2$ (2M+Na); Anal. calcd for $\mathrm{C}_{16} \mathrm{H}_{30} \mathrm{~N}_{2} \mathrm{O}_{4}$ : C, 61.12; H, 9.62; N, 8.91. Found: C, 61.26; H, 9.73; N, 8.91.

\section{$N$-(Tetrahydro-furan-3-yl)- $N$ '-(tert-butoxycarbonyl)hydrazinecarboxylic acid tert-butyl ester (188)}

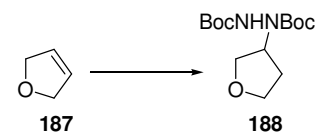

Following GP1 Hydrohydrazination product $188(47 \mathrm{mg}, 0.16 \mathrm{mmol}, 31 \%)$ was obtained as a colorless solid with 2,4dehydrofuran (187) $(38 \mu \mathrm{L}, 0.50 \mathrm{mmol}, 1.0$ equiv) in $12 \mathrm{~h}$ with $5 \mathrm{~mol} \%$ catalyst 20 after purification by column chromatography (pentane $/ \mathrm{CH}_{2} \mathrm{Cl}_{2} / \mathrm{Et}_{2} \mathrm{O}$ 2:1:1). Following GP3 Hydrohydrazination product $\mathbf{1 8 8}(123 \mathrm{mg}, 0.407 \mathrm{mmol}, 81 \%)$ was obtained as a colorless solid with 2,4-dehydrofuran (187) $(38 \mu \mathrm{L}, 0.50 \mathrm{mmol}, 1.0$ equiv) in $4 \mathrm{~h}$ with 2 mol\% catalyst 23 after purification by

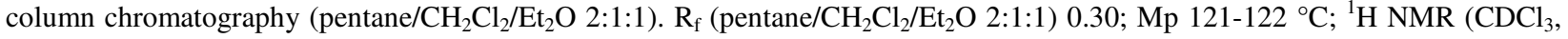
$\left.300 \mathrm{MHz}, 52{ }^{\circ} \mathrm{C}\right) \delta 6.36$ (br s, $\left.1 \mathrm{H}, \mathrm{NH}\right), 4.72$ (br s, $\left.1 \mathrm{H}, \mathrm{CHN}\right), 3.93-3.68\left(\mathrm{~m}, 4 \mathrm{H}, \mathrm{CH}_{2} \mathrm{O}\right), 2.07-2.03\left(\mathrm{~m}, 2 \mathrm{H}, \mathrm{C}_{2} \mathrm{CH}_{2}\right), 1.44(\mathrm{~s}, 9 \mathrm{H}$, $\left.\mathrm{OCCH}_{3}\right), 1.43\left(\mathrm{~s}, 9 \mathrm{H}, \mathrm{OCCH}_{3}\right) ;{ }^{13} \mathrm{C} \mathrm{NMR}\left(\mathrm{CDCl}_{3}, 75 \mathrm{MHz}, 52{ }^{\circ} \mathrm{C}\right) \delta 155.8,154.8,81.4,70.3,67.5,57.7,29.6,28.1,28.1 ; \mathrm{IR}$ $(\mathrm{KBr}) \vee 3314(\mathrm{~m}), 2980(\mathrm{~m}), 2935(\mathrm{w}), 2872(\mathrm{w}), 1704(\mathrm{~s}), 1518(\mathrm{~m}), 1458(\mathrm{w}), 1407(\mathrm{~m}), 1367(\mathrm{~s}), 1344(\mathrm{~m}), 1299(\mathrm{~m}), 1254(\mathrm{~s})$, 1160 (s), $1083(\mathrm{~m}), 1024(\mathrm{w}), 951(\mathrm{~m}), 918(\mathrm{w}), 857(\mathrm{w}), 785(\mathrm{w}), 757(\mathrm{~m}), 616(\mathrm{w}), 463(\mathrm{w})$; MS (ESI) $303.1(\mathrm{M}+\mathrm{H}), 325.0$ $(\mathrm{M}+\mathrm{Na})$; Anal. calcd for $\mathrm{C}_{14} \mathrm{H}_{26} \mathrm{~N}_{2} \mathrm{O}_{5}$ : C, 55.61; H, 8.67; N, 9.26. Found: C, 55.34; H, 8.81; N, 9.16.

$N$-(1,1,2-Trimethyl-ethyl)- $N$ '-(tert-butoxycarbonyl)hydrazinecarboxylic acid tert-butyl ester (190)

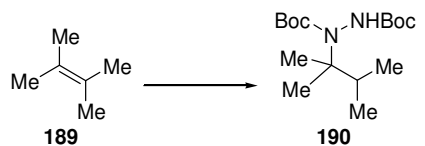

Following GP1 Hydrohydrazination product $190(22 \mathrm{mg}, 0.070 \mathrm{mmol}, 14 \%)$ was obtained as a colorless solid with 2,3dimethylbutene (189) $(60 \mu \mathrm{L}, 0.50 \mathrm{mmol}, 1.0$ equiv) in $10 \mathrm{~h}$ with 5 mol\% catalyst 20 after purification by column chromatography (AcOEt/hexane 1:15). Following GP3 Hydrohydrazination product 190 (123 mg, 0.389 mmol, 78\%) was obtained as a colorless solid with 2,3-dimethylbutene (189) $(60 \mu \mathrm{L}, 0.50 \mathrm{mmol}, 1.0$ equiv) in $3 \mathrm{~h}$ with 2 mol\% catalyst $\mathbf{2 3}$ after purification by column chromatography (AcOEt/hexane 1:15). Following GP3, but with PMHS Hydrohydrazination product 190 $(94 \mathrm{mg}, 0.30 \mathrm{mmol}, 60 \%)$ was obtained as colorless solid with 2,3-dimethylbutene (189) (60 $\mu \mathrm{L}, 0.50 \mathrm{mmol}, 1.0$ equiv) and PMHS $\left(0.10 \mathrm{~mL}, 1.6 \mathrm{H}\right.$ equiv) in $10 \mathrm{~h}$ at $23{ }^{\circ} \mathrm{C}$ with $2 \mathrm{~mol} \%$ catalyst 23 after purification by column chromatography (AcOEt/hexane 1:15). $\mathrm{R}_{\mathrm{f}}$ (AcOEt/hexane 1:5) 0.60; Mp 101-102 ${ }^{\circ} \mathrm{C} ;{ }^{1} \mathrm{H} \mathrm{NMR}\left(\mathrm{CDCl}_{3}, 300 \mathrm{MHz}, 52{ }^{\circ} \mathrm{C}\right) \delta 6.03(\mathrm{br} \mathrm{s}, 1 \mathrm{H}, \mathrm{NH})$, 2.57 (septet, $\left.J=6.9 \mathrm{~Hz}, 1 \mathrm{H}, \mathrm{CHCH}_{3}\right), 1.47\left(\mathrm{~s}, 3 \mathrm{H}, \mathrm{CNCH}_{3}\right), 1.45\left(\mathrm{~s}, 9 \mathrm{H}, \mathrm{OCCH}_{3}\right), 1.43\left(\mathrm{~s}, 9 \mathrm{H}, \mathrm{OCCH}_{3}\right), 1.09\left(\mathrm{~s}, 3 \mathrm{H}, \mathrm{CNCH}_{3}\right)$, $0.85\left(\mathrm{~d}, J=6.9 \mathrm{~Hz}, 3 \mathrm{H}, \mathrm{CHCH}_{3}\right), 0.79\left(\mathrm{~d}, J=6.9 \mathrm{~Hz}, 3 \mathrm{H}, \mathrm{CHCH}_{3}\right) ;{ }^{13} \mathrm{C} \mathrm{NMR}\left(\mathrm{CDCl}_{3}, 75 \mathrm{MHz}, 52{ }^{\circ} \mathrm{C}\right) \delta 156.1,154.5,80.5,65.8$, 34.0, 28.4, 28.3, 24.9, 20.8, 18.1, 17.8; IR v 3339 (m), 3268 (m), 3158 (w), 2977 (s), 2934 (s), 2879 (m), 1714 (s), 1477 (s), 1456 (s), 1367 (s), 1252 (s), 1171 (s), 1082 (s), 1065 (s), 1046 (m), 1018 (s), 908 (m), 889 (w), $858(\mathrm{~m}), 835$ (w), 785 (m), 762 (m), 735 $(\mathrm{m}), 710(\mathrm{w}), 593(\mathrm{w}), 462(\mathrm{w})$; MS (ESI) $317.1(\mathrm{M}+\mathrm{H})$; Anal. calcd for $\mathrm{C}_{16} \mathrm{H}_{32} \mathrm{~N}_{2} \mathrm{O}_{4}$ : C, 60.73; H, 10.19; N, 8.85. Found: C, $60.51 ; \mathrm{H}, 10.45 ; \mathrm{N}, 8.72$.

$N$-(1,2-Dimethyl-cyclohexyl)-N'-(tert-butoxycarbonyl)hydrazinecarboxylic acid tert-butyl ester (192)

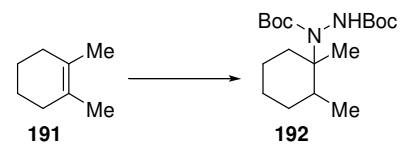

Following GP1 Hydrohydrazination product $192(30 \mathrm{mg}, 0.088 \mathrm{mmol}, 17 \%, 1: 1$ mixture of diastereoisomers) was obtained as a colorless solid with 1,2-dimethyl-cyclohexene (191) (57 mg, $0.52 \mathrm{mmol}, 1.0$ equiv, contains < 17\% 1,6-dimethyl-cyclohexene) in $20 \mathrm{~h}$ with $5 \mathrm{~mol} \%$ catalyst $\mathbf{2 0}$ after purification by column chromatography (AcOEt/hexane 1:15). Following GP3 Hydrohydrazination product $192(144 \mathrm{mg}, 0.420 \mathrm{mmol}, 79 \%, 1: 1$ mixture of diastereoisomers) was obtained as a colorless solid with 1,2-dimethyl-cyclohexene (191) (58 mg, $0.53 \mathrm{mmol}, 1.0$ equiv, contains < 17\% 1,6-dimethyl-cyclohexene) in $3 \mathrm{~h}$ with 2 
mol\% catalyst $\mathbf{2 3}$ after purification by column chromatography (AcOEt/hexane 1:15). $\mathrm{R}_{\mathrm{f}}(\mathrm{AcOEt} / \mathrm{hexane} 1: 5)$ 0.50; Mp 97-99 ${ }^{\circ} \mathrm{C}$; ${ }^{1} \mathrm{H}$ NMR $\left(\mathrm{CDCl}_{3}, 300 \mathrm{MHz}, 52{ }^{\circ} \mathrm{C}\right) \delta 6.07$ (br s, $2 \mathrm{H}, \mathrm{NH}$ (2 diastereoisomers)), 2.70-2.45 (m, 3H, $\mathrm{CHCH}_{3}$ and $\left.\mathrm{CH}_{2}\right), 1.92-1.11$ $\left(\mathrm{m}, 15 \mathrm{H}, \mathrm{CHCH}_{3}\right.$ and $\left.\mathrm{CH}_{2}\right), 1.48\left(\mathrm{~s}, 9 \mathrm{H}, \mathrm{OCCH}_{3}\right), 1.48\left(\mathrm{~s}, 9 \mathrm{H}, \mathrm{OCCH}_{3}\right), 1.46\left(\mathrm{~s}, 9 \mathrm{H}, \mathrm{OCCH}_{3}\right), 1.46(\mathrm{~s}, 9 \mathrm{H}, \mathrm{OCCH}), 1.29(\mathrm{~s}, 3 \mathrm{H}$, $\left.\mathrm{CNCH}_{3}\right), 1.17\left(\mathrm{~s}, 3 \mathrm{H}, \mathrm{CNCH}_{3}\right), 0.88\left(\mathrm{~d}, J=6.9 \mathrm{~Hz}, 3 \mathrm{H}, \mathrm{CHCH}_{3}\right), 0.78\left(\mathrm{~d}, J=6.9 \mathrm{~Hz}, 3 \mathrm{H}, \mathrm{CHCH}_{3}\right) ;{ }^{13} \mathrm{C} \mathrm{NMR}\left(\mathrm{CDCl}_{3}, 75 \mathrm{MHz}\right.$, $\left.52{ }^{\circ} \mathrm{C}\right) \delta 156.0,154.7,80.4,66.9,66.4,40.0,35.5,35.4,35.2,32.1,31.8,28.4,28.4,28.3,25.5,25.2,23.7,23.3,18.0,17.0,16.5$, 16.3; IR v 3340 (w), $3267(\mathrm{w}), 3156$ (w), 2977 (m), 2929 (m), 2862 (w), 1705 (s), 1477 (m), 1458 (m), 1391 (s), 1367 (s), 1340 (m), $1329(\mathrm{~m}), 1285(\mathrm{~m}), 1252(\mathrm{~m}), 1172(\mathrm{~s}), 1092(\mathrm{~m}), 1074(\mathrm{~m}), 1046(\mathrm{~m}), 1017(\mathrm{~m}), 894(\mathrm{w}), 862(\mathrm{w}), 783(\mathrm{w}), 761(\mathrm{w}), 701$ (w), $621(w)$; MS (ESI) $343.2(\mathrm{M}+\mathrm{H})$; Anal. calcd for $\mathrm{C}_{18} \mathrm{H}_{34} \mathrm{~N}_{2} \mathrm{O}_{4}$ : C, 63.13; H, 10.01; N, 8.18. Found: C, 63.04; H, 10.05; N, 8.05 .

Octahydro-naphthalen-4a-yl)- $N$ '-(tert-butoxycarbonyl)hydrazinecarboxylic acid-tert-butyl ester (194)

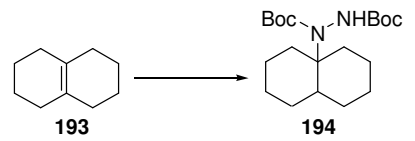

Following GP1 Hydrohydrazination product $194(18 \mathrm{mg}, 0.049 \mathrm{mmol}, 10 \%)$ was obtained as a colorless solid with octalin 193 (70 mg, $0.51 \mathrm{mmol}, 1.0$ equiv, contains $<7 \%$ trisubstituted olefin) in $18 \mathrm{~h}$ with $5 \mathrm{~mol} \%$ catalyst 20 after purification by column chromatography (AcOEt/hexane 1:15). Following GP3 Hydrohydrazination product 194 (140 mg, 0.380 mmol, 74\%) was obtained as a colorless solid with octalin $193(70 \mathrm{mg}, 0.51 \mathrm{mmol}, 1.0$ equiv, contains < 7\% trisubstituted olefin) in $18 \mathrm{~h}$ with 2

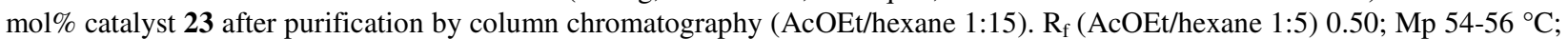
${ }^{1} \mathrm{H} \mathrm{NMR}\left(\mathrm{CDCl}_{3}, 300 \mathrm{MHz}, 52{ }^{\circ} \mathrm{C}\right) \delta 6.12$ (br s, $\left.1 \mathrm{H}, \mathrm{NH}\right), 2.51$ (br s, $\left.1 \mathrm{H}, \mathrm{CH}\right), 2.20-1.96\left(\mathrm{~m}, 2 \mathrm{H}, \mathrm{CH}_{2}\right), 1.80-1.72\left(\mathrm{~m}, 2 \mathrm{H}, \mathrm{CH}_{2}\right)$, $1.60-1.23\left(\mathrm{~m}, 12 \mathrm{H}, \mathrm{CH}_{2}\right), 1.47\left(\mathrm{~s}, 9 \mathrm{H}, \mathrm{OCCH}_{3}\right), 1.44\left(\mathrm{~s}, 9 \mathrm{H}, \mathrm{OCCH}_{3}\right) ;{ }^{13} \mathrm{C} \mathrm{NMR}\left(\mathrm{CDCl}_{3}, 75 \mathrm{MHz}, 52{ }^{\circ} \mathrm{C}\right) \delta 156.2,154.7,80.4$, 77.2, 66.2, 36.6, 30.9, 29.0, 28.8, 28.4, 28.2, 23.2, 23.0; IR v $3331(\mathrm{w}), 3261(\mathrm{w}), 3156(\mathrm{w}), 2977(\mathrm{~s}), 2930(\mathrm{~s}), 2865(\mathrm{~s}), 1749(\mathrm{~s})$, 1704 (s), 1478 (m), 1454 (m), 1392 (s), 1367 (s), 1328 (s), 1308 (m), 1292 (m), 1253 (s), 1161 (s), 1104 (m), 1081 (m), 1047 (m), $1020(\mathrm{~m}), 978(\mathrm{~m}), 914(\mathrm{~m}), 857(\mathrm{w}), 762(\mathrm{w}), 734(\mathrm{~m}), 647(\mathrm{w}), 618(\mathrm{w}), 463(\mathrm{w})$; MS (ESI) 369.2 (M+H); Anal. calcd for $\mathrm{C}_{20} \mathrm{H}_{36} \mathrm{~N}_{2} \mathrm{O}_{4}$ : C, 65.19; H, 9.85; N, 7.60. Found: C, 65.03; H, 9.85; N, 7.41.

$N$-(1,2-Dimethyl-1-phenyl-propyl)- $N$ '-(tert-butoxycarbonyl)hydrazinecarboxylic acid tert-butyl ester (196)

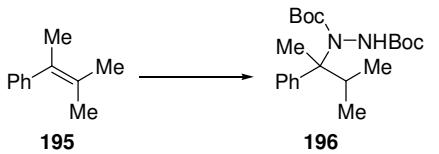

Following GP1 Hydrohydrazination product $196(24 \mathrm{mg}, 0.063 \mathrm{mmol}, 13 \%)$ was obtained as a gel with 2-methyl-1phenylbutene (195) (71 mg, $0.49 \mathrm{mmol}, 1.0$ equiv) in $20 \mathrm{~h}$ with $5 \mathrm{~mol} \%$ catalyst 20 after purification by column chromatography (AcOEt/hexane 1:10). Following GP3 Hydrohydrazination product 196 (94 mg, $0.25 \mathrm{mmol}, 51 \%$ ) was obtained as a gel with 2methyl-1-phenylbutene (195) (71 mg, $0.49 \mathrm{mmol}, 1.0$ equiv) in $4 \mathrm{~h}$ with 2 mol\% catalyst 23 after purification by column chromatography (AcOEt/hexane 1:10). $\mathrm{R}_{\mathrm{f}}$ (AcOEt/hexane 1:5) $0.50 ;{ }^{1} \mathrm{H} \mathrm{NMR}\left(\mathrm{CDCl}_{3}, 300 \mathrm{MHz}, 52{ }^{\circ} \mathrm{C}\right.$, mixture of rotamers major/minor: 7:3) $\delta$ 7.50-7.47 (m, 2H, Ar H), 7.30-7.13 (m, 3H, Ar H), 6.19-5.95 (br m, 1H, NH), 2.95 (br s, 0.3H, CH, minor rotamer), 2.78 (quintet, $J=6.9 \mathrm{~Hz}, 0.7 \mathrm{H}, \mathrm{CH}$, major rotamer), $1.61\left(\mathrm{~s}, 0.9 \mathrm{H}, \mathrm{CNCH}_{3}\right.$, minor rotamer), $1.58\left(\mathrm{~s}, 2.7 \mathrm{H}, \mathrm{OCCH}_{3}\right.$, minor rotamer), $1.52\left(\mathrm{~s}, 6.3 \mathrm{H}, \mathrm{OCCH}_{3}\right.$, major rotamer), $1.49\left(\mathrm{~s}, 2.1 \mathrm{H}, \mathrm{CNCH}_{3}\right.$, major rotamer), $1.28\left(\mathrm{~s}, 2.7 \mathrm{H}, \mathrm{OCCH}_{3}, \mathrm{minor}\right.$ rotamer), $1.15\left(\mathrm{~s}, 6.3 \mathrm{H}, \mathrm{OCCH}_{3}\right.$, major rotamer), $1.00\left(\mathrm{~d}, J=6.9 \mathrm{~Hz}, 0.9 \mathrm{H}, \mathrm{CHCH}_{3}\right.$, minor rotamer $), 0.92-0.87(\mathrm{~m}, 4.2 \mathrm{H}, \mathrm{CHCH}$, major rotamer), $0.69(\mathrm{~d}, J=6.9 \mathrm{~Hz}, 0.9 \mathrm{H}$, minor rotamer $) ;{ }^{13} \mathrm{C} \mathrm{NMR}\left(\mathrm{CDCl}_{3}, 75 \mathrm{MHz}, 52{ }^{\circ} \mathrm{C}\right) \delta 156.4,155.1,154.8,146.3$, 144.4, 127.4, 127.3, 126.5, 126.2, 125.7, 81.0, 70.9, 69.4, 34.7, 34.0, 28.4, 28.1, 28.0, 21.8, 19.8, 19.1, 18.9, 18.3; IR v 3263 (m), $3090(\mathrm{w}), 3058(\mathrm{w}), 2978(\mathrm{~s}), 2933(\mathrm{~m}), 2882(\mathrm{~m}), 1711(\mathrm{~s}), 1602(\mathrm{w}), 1495(\mathrm{~m}), 1478(\mathrm{~m}), 1455(\mathrm{~m}), 1392(\mathrm{~s}), 1367(\mathrm{~s}), 1248(\mathrm{~s})$, $1163(\mathrm{~s}), 1093(\mathrm{~m}), 1047(\mathrm{~m}), 1018(\mathrm{~m}), 911(\mathrm{~m}), 855(\mathrm{~m}), 759(\mathrm{~m}), 734(\mathrm{~s}), 703(\mathrm{~s}), 646$ (w), 609 (w); MS (ESI) 401.2 (M+Na); Anal. calcd for $\mathrm{C}_{21} \mathrm{H}_{34} \mathrm{~N}_{2} \mathrm{O}_{4}$ : C, 66.64; H, 9.05; N, 7.40. Found: C, 66.44; H, 9.01; N, 7.25.

2-(N,N'-Di-tert-butoxycarbonyl-hydrazino)-butyric acid 4,4-dimethyl-2-oxo-tetrahydro-furan-(R)-3-yl ester (26)
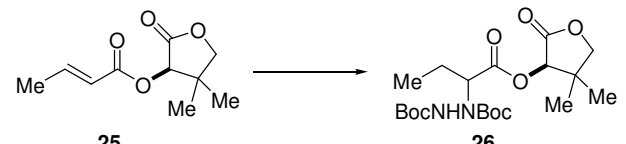

Following GP1 Hydrohydrazination product 26 (156 mg, $0.362 \mathrm{mmol}, 74 \%, 78: 22$ mixture of diastereoisomers by integration of the singulet at 5.30 respectively 5.33 in the ${ }^{1} \mathrm{H}$-NMR) was obtained as a colorless solid with olefin 25 (97 mg, $0.49 \mathrm{mmol}, 1.0$ equiv) in $20 \mathrm{~h}$ with $5 \mathrm{~mol} \%$ catalyst 20 after purification by column chromatography ( $\mathrm{AcOEt} / \mathrm{CH}_{2} \mathrm{Cl}_{2}$ 1:50). Following GP3 Hydrohydrazination product 26 (145 mg, $0.337 \mathrm{mmol}, 75 \%, 81: 19$ mixture of diastereoisomers by integration of the singulet at 5.30 respectively 5.33 in the ${ }^{1} \mathrm{H}$-NMR) was obtained as a colorless solid with olefin $\mathbf{2 5}(90 \mathrm{mg}, 0.45 \mathrm{mmol}, 1.0$ equiv) in $3 \mathrm{~h}$ with $2.5 \mathrm{~mol} \%$ catalyst 23 after purification by column chromatography $\left(\mathrm{AcOEt} / \mathrm{CH}_{2} \mathrm{Cl}_{2}\right.$ 1:50). $\mathrm{R}_{\mathrm{f}}\left(\mathrm{AcOEt} / \mathrm{CH}_{2} \mathrm{Cl} \mathbf{2}_{1: 20)} \mathbf{0 . 4 0 ;} \mathrm{Mp}\right.$ $107-108{ }^{\circ} \mathrm{C} ;{ }^{1} \mathrm{H} \mathrm{NMR}\left(\mathrm{CDCl}_{3}, 300 \mathrm{MHz}, 52{ }^{\circ} \mathrm{C}\right) \delta 6.34$ (br s, $\left.1 \mathrm{H}, \mathrm{NH}\right), 5.33\left(\mathrm{~s}, 1 \mathrm{H}, \mathrm{C}_{2} \mathrm{CHO}\right.$, minor diastereoisomer), $5.30(\mathrm{~s}, 1 \mathrm{H}$, $\mathrm{C}_{2} \mathrm{CHO}$, major diastereoisomer), 4.68 (br m, $\left.1 \mathrm{H}, \mathrm{CHN}\right), 4.04-3.97\left(\mathrm{~m}, 2 \mathrm{H}, \mathrm{CH}_{2} \mathrm{O}\right), 2.01-1.81\left(\mathrm{~m}, 2 \mathrm{H}, \mathrm{CH}_{2} \mathrm{CH}_{3}\right), 1.45(\mathrm{~s}, 9 \mathrm{H}$, $\left.\mathrm{OC}\left(\mathrm{CH}_{3}\right)_{3}\right), 1.45\left(\mathrm{~s}, 9 \mathrm{H}, \mathrm{OC}\left(\mathrm{CH}_{3}\right)_{3}\right), 1.20\left(\mathrm{~s}, 3 \mathrm{H}, \mathrm{C}\left(\mathrm{CH}_{3}\right)_{2}\right), 1.11\left(\mathrm{~s}, 3 \mathrm{H}, \mathrm{C}\left(\mathrm{CH}_{3}\right)_{2}\right), 1.16-1.06\left(\mathrm{~m}, 3 \mathrm{H}, \mathrm{CH}_{2} \mathrm{CH}_{3}\right) ;{ }^{13} \mathrm{C} \mathrm{NMR}$ $\left(\mathrm{CDCl}_{3}, 75 \mathrm{MHz}, 52{ }^{\circ} \mathrm{C}\right) \delta 171.4,171.2,155.3,155.1,82.1,81.0,76.3,76,2,75.7,61.7,40.3,40.2,29.7,28.2,28.2,28.0,23.0$, 
22.9, 22.4, 19.9, 11.1; IR v $3337(\mathrm{~m}), 2979(\mathrm{~s}), 2936(\mathrm{~m}), 2881(\mathrm{~m}), 1795(\mathrm{~s}), 1753(\mathrm{~s}), 1480(\mathrm{~s}), 1393$ (s), 1369 (s), 1299 (s), 1255 (s), 1156 (s), 1112 (s), 1089 (s), 1033 (m), 1014 (m), 998 (m), 943 (m), 917 (m), 856 (m), 770 (m), 734 (m), 648 (w), 562 (w), $544(w)$; MS (ESI) $431.3(\mathrm{M}+\mathrm{H}), 453.2(\mathrm{M}+\mathrm{Na}), 469.1(\mathrm{M}+2 \mathrm{Na})$; Anal. calcd for $\mathrm{C}_{20} \mathrm{H}_{34} \mathrm{~N}_{2} \mathrm{O}_{8}$ : C, 55.80; H, 7.96; N, 6.51. Found: C, 55.54; H, 7.90; N, 6.40 .

\section{The Co-Catalyzed Hydroazidation of Olefins}

\subsection{Catalysts and Azide Transfer Reagents}

The Co-salen catalysts described in this work (28-31) were all prepared using the same known procedure, ${ }^{29}$ and were used as obtained without further purification and characterization. Co catalysts $\mathbf{3 2} \mathbf{a}-\mathbf{3 2} \mathbf{g}$ were synthesized following the same procedure as for $\mathbf{2 0}$ and were used as obtained without further purification and characterization.

Potassium\{[1-(3,5-di-tert-butyl-2-hydroxy-phenyl)-meth-(E)-ylidene]-amino\}-diphenyl-acetate (37) and [N-3,5-di-tertbutyl-salicylidene-2,2-diphenyl-glycinato]- cobalt (III) (36)

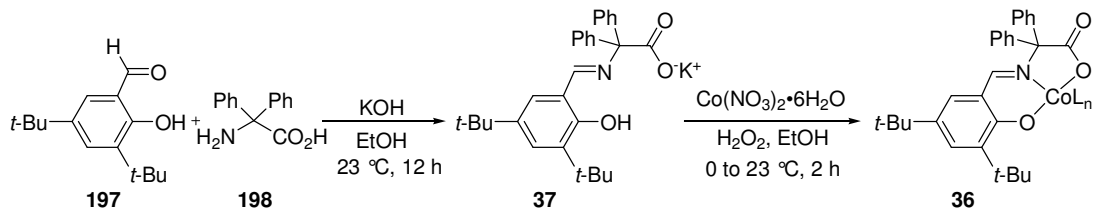

Potassium hydroxide (0.5 M in EtOH, $9.3 \mathrm{~mL}, 4.6 \mathrm{mmol}, 1.1$ equiv) was added to a suspension of $\alpha, \alpha$-diphenyl glycine (198) $(1.0 \mathrm{~g}, 4.4 \mathrm{mmol}, 1.1 \mathrm{equiv})$ in ethanol $(50 \mathrm{~mL})$ at $23{ }^{\circ} \mathrm{C}$ under argon. After $30 \mathrm{~min}, 3,5$-di-tert-butyl-salicylaldehyde $(\mathbf{1 9 7})(1.0 \mathrm{~g}$, $4.4 \mathrm{mmol}, 1.0$ equiv) was added to the clear colorless solution, and the color changed to intensive yellow. The reaction mixture was stirred at $23{ }^{\circ} \mathrm{C}$ for $10 \mathrm{~h}$ and the solvent was removed under reduced pressure. The isolated hygroscopic yellow solid was further dried in high vacuo for $8 \mathrm{~h}$ to furnish $\mathbf{4 3}(2.2 \mathrm{~g}, 4.5 \mathrm{mmol}, 100 \%)$, which was used without further purifications. A solution of $\mathrm{Co}\left(\mathrm{NO}_{3}\right)_{2} \cdot 6 \mathrm{H}_{2} \mathrm{O}(61 \mathrm{mg}, 0.21 \mathrm{mmol}, 1.0$ equiv) in ethanol $(1 \mathrm{~mL})$ was added to a solution of ligand $\mathbf{4 3}(100 \mathrm{mg}, 0.21 \mathrm{mmol}$, 1.0 equiv) in ethanol $(0.5 \mathrm{~mL})$ and $\mathrm{CH}_{2} \mathrm{Cl}_{2}(1.5 \mathrm{~mL})$ at $0{ }^{\circ} \mathrm{C}$ under argon, whereas an orange solid precipitated. The reaction mixture was warmed to $23{ }^{\circ} \mathrm{C}$, stirred for $6 \mathrm{~h}$ and hydrogen peroxide $\left(43 \mu \mathrm{L}, 0.42 \mathrm{mmol}, 2.0\right.$ equiv) was added dropwise at $0{ }^{\circ} \mathrm{C}$. After $30 \mathrm{~min}$, the reaction mixture was warmed to $23{ }^{\circ} \mathrm{C}$, stirred for $1.5 \mathrm{~h}$ and the dark red solution was filtered. The solvent was removed under reduced pressure, co-evaporated with $\mathrm{CH}_{2} \mathrm{Cl}_{2}(2 \mathrm{x})$, the residues were dissolved in $\mathrm{CH}_{2} \mathrm{Cl}_{2}(10 \mathrm{~mL})$ and filtered. The solvent was removed under reduced pressure and the remaining solid dissolved in $\mathrm{Et}_{2} \mathrm{O}(20 \mathrm{~mL})$ and filtered. The solvent was removed under reduced pressure and the obtained dark red solid triturated in hexane $(30 \mathrm{~mL})$, filtered, washed with hexane $(10$ $\mathrm{mL})$ and dried $12 \mathrm{~h}$ in high vacuo to afford a dark red solid $(84 \mathrm{mg})$, which appear to be the nearly pure $1: 1$ complex $\mathbf{4 2}$ by ${ }^{1} H$ NMR, the coordination sphere of the complex being probably completed with water. ${ }^{1} \mathrm{H}$ NMR (DMSO- $\left.\mathrm{d}_{6}, 300 \mathrm{MHz}\right) \delta 8.03(\mathrm{~s}$, $1 \mathrm{H}$, imine H), 7.48-7.41 (m, 4H, Ar H), 7.27-7.20 (m, 6H, ArH), 6.87-6.85 (m, 2H, Ar H), 3.53 (br s, 5 to 10H, $\mathrm{H}_{2} \mathrm{O}$ ), 1.08 (s, 9H, $\left.\mathrm{CCH}_{3}\right), 0.70\left(\mathrm{~s}, 9 \mathrm{H}, \mathrm{CCH}_{3}\right) ;{ }^{13} \mathrm{C}$ NMR $\left(\mathrm{DMSO}-\mathrm{d}_{6}, 75 \mathrm{MHz}\right)^{30} \delta 168.9,163.1,143.8,143.1,140.9,133.4,130.0,129.7,129.6$, 128.4, 127.6, 127.5, 127.0, 126.9, 126.6, 118.9, 82.5, 34.6, 33.2, 31.2, 29.5; IR (KBr) v $3390(\mathrm{~m}), 2955(\mathrm{~m}), 1762(\mathrm{w}), 1635(\mathrm{~s})$, $1527(\mathrm{w}), 1431(\mathrm{~m}), 1385$ (s), $1270(\mathrm{w}), 1254(\mathrm{w}), 1200(\mathrm{w}), 1169(\mathrm{w}), 1090(\mathrm{w}), 1049(\mathrm{w}), 1027(\mathrm{w}), 916(\mathrm{w}), 870(\mathrm{w}), 844(\mathrm{w})$, 826 (w), 743 (w), 700 (m), 595 (w), 545 (w); HRMS (MALDI) calcd for $\mathrm{C}_{29} \mathrm{H}_{31} \mathrm{CoNNaO}_{3}{ }^{+}$(M+Na): 523.1528, found 523.1519.

Ethanesulfonyl azide (200) ${ }^{31}$

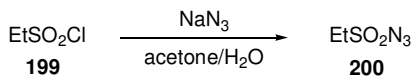

Following a reported procedure,${ }^{31}$ a solution of sodium azide $(9.5 \mathrm{~g}, 0.15 \mathrm{~mol}, 1.5$ equiv) in water $(60 \mathrm{~mL})$ was added dropwise over $1 \mathrm{~h}$ to a solution of ethanesulfonyl chloride $(\mathbf{1 9 9})\left(8.9 \mathrm{~mL}, 93 \mathrm{mmol}, 1.0\right.$ equiv) in acetone $(200 \mathrm{~mL})$ at $0{ }^{\circ} \mathrm{C}$. The reaction was let to warm up to $23{ }^{\circ} \mathrm{C}$ and stirred for $11 \mathrm{~h}$, the acetone was removed under reduced pressure at $25{ }^{\circ} \mathrm{C}$ and the reaction mixture was extracted with AcOEt $(2 \times 100 \mathrm{~mL})$. The combined organic layers were washed with water $(2 \times 100 \mathrm{~mL}), 5 \% \mathrm{Na}_{2} \mathrm{CO}_{3}$ $(2 \times 100 \mathrm{~mL})$ and water $(2 \times 100 \mathrm{~mL})$, dried over $\mathrm{Na}_{2} \mathrm{SO}_{4}$ and the solvent was removed under reduced pressure. The crude product was purified by distillation $\left(p=0.6 \mathrm{mbar}, b p=45-48{ }^{\circ} \mathrm{C}\right)$ to afford ethanesulfonyl azide (200) $(10.5 \mathrm{~g}, 7.77 \mathrm{mmol}, 83 \%)$ as a colorless liquid. ${ }^{1} \mathrm{H} \mathrm{NMR}\left(\mathrm{CDCl}_{3}, 300 \mathrm{MHz}\right) \delta 3.33\left(\mathrm{q}, J=7.2 \mathrm{~Hz}, 2 \mathrm{H}, \mathrm{CH}_{2}\right), 1.45\left(\mathrm{t}, J=7.2 \mathrm{~Hz}, 3 \mathrm{H}, \mathrm{CH}_{3}\right) ;{ }^{13} \mathrm{C} \mathrm{NMR}\left(\mathrm{CDCl}_{3}, 75\right.$ MHz) $\delta$ 50.4, 8.0; IR v 3309 (w), 2987 (w), 2948 (w), 2886 (w), 2369 (w), 2138 (s), 1458 (m), 1411 (w), 1360 (s), 1289 (w), 1199 (s), $1159(\mathrm{~s}), 1050(\mathrm{~m}), 978(\mathrm{w}), 783(\mathrm{~s}), 745(\mathrm{~m}), 613(\mathrm{~m}), 568(\mathrm{~m}), 519(\mathrm{~m})$.

Methanesulfonyl azide (201), benzenesulfonyl azide (202), toluenesulfonyl azide (203) mesitylenesulfonyl azide (204), $p$-nitrotoluenesulfonyl azide (205), p-methoxy-benzenesulfonyl azide (42) and camphor-derived sulfonyl azide 41 were synthezised following the same procedure, ${ }^{31}$ but distillation was omitted, as these compounds were already pure enough.

Methanesulfonyl azide (201) $)^{32}$

${ }^{1} \mathrm{H}$ NMR $\left(\mathrm{CDCl}_{3}, 300 \mathrm{MHz}\right) \delta 3.24\left(\mathrm{~s}, 3 \mathrm{H}, \mathrm{CH}_{3}\right) ;{ }^{13} \mathrm{C} \mathrm{NMR}\left(\mathrm{CDCl}_{3}, 75 \mathrm{MHz}\right) \delta 42.6$; IR v $3318(\mathrm{w}), 3036(\mathrm{w}), 2938(\mathrm{w}), 2141$ (s), $1413(\mathrm{w}), 1360$ (s), $1167(\mathrm{~s}), 968(\mathrm{~m}), 781(\mathrm{~s}), 730(\mathrm{~m}), 574(\mathrm{~s}), 509(\mathrm{~s})$. 


\section{Benzenesulfonyl azide (202) $)^{31}$}

${ }^{1} \mathrm{H}$ NMR $\left(\mathrm{CDCl}_{3}, 300 \mathrm{MHz}\right) \delta$ 7.96-7.92 (m, 2H, Ar H), 7.75-7.69 (m, 1H, Ar H), 7.64-7.57 (m, 2H, Ar H); ${ }^{13} \mathrm{C} \mathrm{NMR}\left(\mathrm{CDCl}{ }_{3}\right.$, $75 \mathrm{MHz}) \delta$ 138.1, 134.6, 129.5, 127.2; IR v 3276 (w), 3069 (w), 2347 (w), 2129 (s), 1584 (w), 1477 (w), 1450 (m), 1372 (s), 1313 (w), 1298 (w), 1170 (s), 1088 (m), 1023 (w), 1000 (w), 751 (m), 685 (m), 601 (s), 564 (s).

Toluenesulfonyl azide $(\mathbf{2 0 3})^{33}$

${ }^{1} \mathrm{H}$ NMR $\left(\mathrm{CDCl}_{3}, 300 \mathrm{MHz}\right) \delta 7.80(\mathrm{~d}, J=8.4 \mathrm{~Hz}, 2 \mathrm{H}, \mathrm{Ar} \mathrm{H}), 7.38(\mathrm{~d}, J=8.7 \mathrm{~Hz}, 2 \mathrm{H}, \mathrm{Ar} \mathrm{H}), 2.45\left(\mathrm{~s}, 3 \mathrm{H}, \mathrm{CH}_{3}\right) ;{ }^{13} \mathrm{C} \mathrm{NMR}$ $\left(\mathrm{CDCl}_{3}, 75 \mathrm{MHz}\right) \delta$ 146.0, 135.1, 130.0, 127.2, 21.6; IR v $3275(\mathrm{w}), 3068(\mathrm{w}), 2927(\mathrm{w}), 2872(\mathrm{w}), 2351(\mathrm{w}), 2127(\mathrm{~s}), 1924(\mathrm{w})$, $1808(\mathrm{w}), 1596(\mathrm{~m}), 1495(\mathrm{w}), 1451(\mathrm{w}), 1399(\mathrm{w}), 1372(\mathrm{~s}), 1308(\mathrm{w}), 1297(\mathrm{w}), 1168(\mathrm{~s}), 1121(\mathrm{w}), 1086(\mathrm{~m}), 1041(\mathrm{w}), 1018$ (w), $814(\mathrm{~m}), 800(\mathrm{w}), 748(\mathrm{~s}), 703(\mathrm{~m}), 662(\mathrm{~s}), 593(\mathrm{~s}), 540(\mathrm{~s}), 501(\mathrm{w})$.

Mesitylenesulfonyl azide (204) ${ }^{34}$

${ }^{1} \mathrm{H} \mathrm{NMR}\left(\mathrm{CDCl}_{3}, 300 \mathrm{MHz}\right) \delta 7.01(\mathrm{~s}, 2 \mathrm{H}, \mathrm{Ar} \mathrm{H}), 2.65\left(\mathrm{~s}, 6 \mathrm{H}, \mathrm{CH}_{3}\right), 2.33\left(\mathrm{~s}, 3 \mathrm{H}, \mathrm{CH}_{3}\right) ;{ }^{13} \mathrm{C} \mathrm{NMR}\left(\mathrm{CDCl}_{3}, 75 \mathrm{MHz}\right) \delta 144.4$, 139.6, 132.9, 131.9, 22.7, 21.0; IR v 3276 (w), $2982(\mathrm{w}), 2943(\mathrm{w}), 2362(\mathrm{w}), 2123(\mathrm{~s}), 1603(\mathrm{~m}), 1565$ (w), $1455(\mathrm{~m}), 1405$ (m), 1366 (s), 1292 (w), 1278 (w), 1191 (s), 1167 (s), 1052 (m), 1035 (m), 965 (w), 854 (m), 744 (m), 658 (s), 596 (s), 572 (m), 530 (s).

\section{p-Nitro-toluenesulfonyl azide $(\mathbf{2 0 5})^{35}$}

${ }^{1} \mathrm{H} \mathrm{NMR}\left(\mathrm{CDCl}_{3}, 300 \mathrm{MHz}\right) \delta 8.46(\mathrm{~d}, J=9.0 \mathrm{~Hz}, 2 \mathrm{H}, \mathrm{Ar} \mathrm{H}), 8.17(\mathrm{~d}, J=9.0 \mathrm{~Hz}, 2 \mathrm{H}, \mathrm{Ar} \mathrm{H}) ;{ }^{13} \mathrm{C} \mathrm{NMR}\left(\mathrm{CDCl}_{3}, 75 \mathrm{MHz}\right) \delta$ 151.0, 143.5, 128.8, 124.8. IR $v 3107(\mathrm{w}), 2311(\mathrm{w}), 2142(\mathrm{~s}), 1605(\mathrm{w}), 1535(\mathrm{~m}), 1477(\mathrm{w}), 1404(\mathrm{w}), 1369(\mathrm{~m}), 1350(\mathrm{~m}), 1311$ (w), $1177(\mathrm{~s}), 1159$ (s), 1110 (w), 1085 (m), 1013 (w), 907 (w), 868 (w), 854 (m), 770 (m), 744 (m), 732 (m), 681 (m), 607 (s), $585(\mathrm{~s}), 545(\mathrm{~m}), 461(\mathrm{~m})$

\section{p-Methoxy-benzenesulfonyl azide (42) ${ }^{35}$}

${ }^{1} \mathrm{H}$ NMR $\left(\mathrm{CDCl}_{3}, 300 \mathrm{MHz}\right) \delta 7.86(\mathrm{~d}, J=9.0 \mathrm{~Hz}, 2 \mathrm{H}, \mathrm{Ar} \mathrm{H}), 7.04(\mathrm{~d}, J=9.0 \mathrm{~Hz}, 2 \mathrm{H}, \mathrm{Ar} \mathrm{H}), 3.89\left(\mathrm{~s}, 3 \mathrm{H}, \mathrm{CH}_{3}\right) ;{ }^{13} \mathrm{C} \mathrm{NMR}$ $\left(\mathrm{CDCl}_{3}, 75 \mathrm{MHz}\right) \delta 164.4,129.7,129.4,114.7,55.8$; IR v $3276(\mathrm{w}), 3102(\mathrm{w}), 3080(\mathrm{w}), 3015(\mathrm{w}), 2977(\mathrm{w}), 2949(\mathrm{w}), 2845(\mathrm{w})$, 2350 (w), 2128 (s), 1909 (w), 1811 (w), 1772 (w), 1595 (s), 1578 (s), 1499 (s), 1463 (m), 1442 (m), 1418 (m), 1370 (s), 1315 (m), 1267 (s), 1163 (s), $1114(\mathrm{~m}), 1089$ (s), 1023 (m), 915 (w), 836 (s), 806 (s), 748 (s), 715 (w), $668(\mathrm{~m}), 628$ (m), $594(\mathrm{~s}), 551(\mathrm{~s})$, $521(\mathrm{w}), 486(\mathrm{w})$.

(7,7-Dimethyl-2-oxo-bicyclo[2.2.1]hept-1-yl)-methanesulfonyl azide (41) ${ }^{36}$

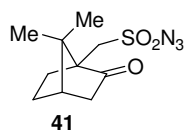

${ }^{1} \mathrm{H}$ NMR $\left(\mathrm{CDCl}_{3}, 300 \mathrm{MHz}\right) \delta 3.79\left(\mathrm{~d}, J=14.9 \mathrm{~Hz}, 1 \mathrm{H}, \mathrm{CH}_{2} \mathrm{SO}_{2} \mathrm{~N}_{3}\right), 3.21\left(\mathrm{~d}, J=14.9 \mathrm{~Hz}, 1 \mathrm{H}, \mathrm{CH}_{2} \mathrm{SO}_{2} \mathrm{~N}_{3}\right), 2.43-2.27(\mathrm{~m}, 2 \mathrm{H}$, $\mathrm{CH}_{2}$ or $\left.\mathrm{CH}\right), 2.27-1.92\left(\mathrm{~m}, 3 \mathrm{H}, \mathrm{CH}_{2}\right.$ or $\left.\mathrm{CH}\right), 1.80-1.51\left(\mathrm{~m}, 1 \mathrm{H}, \mathrm{CH}_{2}\right.$ or $\left.\mathrm{CH}\right), 1.51-1.42\left(\mathrm{~m}, 1 \mathrm{H}, \mathrm{CH}_{2}\right.$ or $\left.\mathrm{CH}\right), 1.06\left(\mathrm{~s}, 3 \mathrm{H}, \mathrm{CH}_{3}\right)$, $0.87\left(\mathrm{~s}, 3 \mathrm{H}, \mathrm{CH}_{3}\right) ;{ }^{13} \mathrm{C} \mathrm{NMR}\left(\mathrm{CDCl}_{3}, 75 \mathrm{MHz}\right) \delta 213.8,58.4,53.4,48.4,42.7,42.4,26.9,24.9,19.7,19.5 ; \mathrm{IR} \vee 2964(\mathrm{~m}), 2892$ (w), $2357(\mathrm{w}), 2139$ (s), 1748 (s), 1472 (w), $1456(\mathrm{w}), 1417$ (w), 1395 (w), 1366 (s), $1280(\mathrm{w}), 1198(\mathrm{~m}), 1162(\mathrm{~s}), 1106(\mathrm{w})$, $1069(\mathrm{w}), 1052(\mathrm{~m}), 1028(\mathrm{w}), 1002(\mathrm{w}), 968(\mathrm{w}), 936(\mathrm{w}), 907(\mathrm{w}), 854(\mathrm{w}), 815(\mathrm{w}), 795(\mathrm{~m}), 734(\mathrm{~m}), 681(\mathrm{w}), 615(\mathrm{~m}), 572$ (m), $545(\mathrm{~m}), 519(\mathrm{~m}), 469(\mathrm{w}), 446(\mathrm{w})$.

Diphenylphosphonyl azide $(\mathbf{4 0})^{37}$

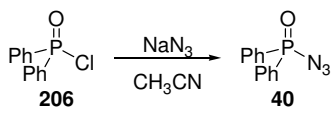

Following a reported procedure, ${ }^{37}$ diphenylphosphonyl chloride (206) (1.0 mL, $5.1 \mathrm{mmol}, 1.0$ equiv) was added dropwise to a suspension of sodium azide $(0.51 \mathrm{~g}, 7.8 \mathrm{mmol}, 1.5 \mathrm{equiv})$ in acetonitrile $(10 \mathrm{~mL})$ at $0{ }^{\circ} \mathrm{C}$ under argon. After stirring at $23{ }^{\circ} \mathrm{C}$ for $12 \mathrm{~h}$, the reaction mixture was filtered, the solvent was removed under reduced pressure at $25{ }^{\circ} \mathrm{C}$ and the reaction mixture was diluted with AcOEt $(20 \mathrm{~mL})$. The organic layer was washed with water $(2 \times 5 \mathrm{~mL}), 5 \% \mathrm{Na}_{2} \mathrm{CO}_{3}(2 \times 5 \mathrm{~mL})$, water $(5 \mathrm{~mL})$ and brine $(5 \mathrm{~mL})$, dried over $\mathrm{Na}_{2} \mathrm{SO}_{4}$, the solvent was removed under reduced pressure and the residues dried $3 \mathrm{~h}$ in high vacuo to yield azide 40 (1.3 g, $5.3 \mathrm{mmol}, 100 \%) .{ }^{1} \mathrm{H} \mathrm{NMR}\left(\mathrm{CDCl}_{3}, 300 \mathrm{MHz}\right) \delta 7.79-7.72(\mathrm{~m}, 6 \mathrm{H}, \mathrm{Ar} \mathrm{H}), 7.53-7.37(\mathrm{~m}, 9 \mathrm{H}, \mathrm{Ar} \mathrm{H}) ;{ }^{13} \mathrm{C} \mathrm{NMR}$ $\left(\mathrm{CDCl}_{3}, 75 \mathrm{MHz}\right) \delta 132.7,132.7,131.1,130.9,130.8,130.5,128.7,128.6,128.4$; IR v $3533(\mathrm{w}), 3059(\mathrm{~m}), 2994(\mathrm{w}), 2507$ (w), 2144 (s), $1971(\mathrm{w}), 1905$ (w), $1824(\mathrm{w}), 1779(\mathrm{w}), 1616(\mathrm{w}), 1591(\mathrm{~m}), 1484(\mathrm{~m}), 1439$ (s), $1392(\mathrm{w}), 1311(\mathrm{~m}), 1262$ (s), 1227 (s), $1184(\mathrm{~m}), 1161(\mathrm{~m}), 1128(\mathrm{~s}), 1107$ (m), $1072(\mathrm{~m}), 1028(\mathrm{w}), 998(\mathrm{~m}), 935(\mathrm{w}), 853(\mathrm{w}), 729(\mathrm{~s}), 694(\mathrm{~s}), 618(\mathrm{~m}), 590(\mathrm{~s})$, $532(\mathrm{~s}), 505(\mathrm{~m})$.

\section{3,5-Dichloro-2-hydroxy-benzenesulfonyl azide (45)}

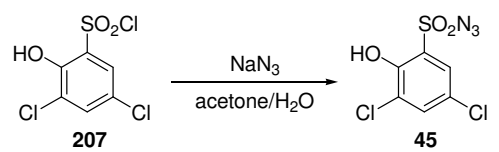

A solution of sodium azide $(0.37 \mathrm{~g}, 5.7 \mathrm{mmol}, 1.5$ equiv) in water $(2.7 \mathrm{~mL})$ was added dropwise over 20 min to a solution of sulfonyl chloride 207 (1.0 g, $3.8 \mathrm{mmol}, 1.0$ equiv) in acetone $(8.7 \mathrm{~mL})$ at $0{ }^{\circ} \mathrm{C}$. The reaction was let to warm up to $23{ }^{\circ} \mathrm{C}$ and 
stirred for $12 \mathrm{~h}$, the acetone was removed under reduced pressure at $25{ }^{\circ} \mathrm{C}$ and the reaction mixture was extracted with AcOEt $(2 \times 30 \mathrm{~mL})$. The combined organic layers were washed with water $(2 \times 20 \mathrm{~mL})$, and brine $(20 \mathrm{~mL})$, dried over $\mathrm{Na}_{2} \mathrm{SO}_{4}$, the solvent was removed under reduced pressure and the residues were dried under high vacuo for $2 \mathrm{~h}$ to afford sulfonyl azide 45 ( $0.55 \mathrm{~g}, 2.1$ mmol, 54\%) as a colorless solid. Mp 94-95 ${ }^{\circ} \mathrm{C} ;{ }^{1} \mathrm{H}$ NMR $\left(\mathrm{CDCl}_{3}, 300 \mathrm{MHz}\right) \delta 7.74(\mathrm{~d}, J=2.5 \mathrm{~Hz}, 1 \mathrm{H}, \mathrm{Ar} \mathrm{H}), 7.67(\mathrm{~d}, J=2.7 \mathrm{~Hz}$, $1 \mathrm{H}, \mathrm{Ar} \mathrm{H}), 7.50$ (s, 1H, OH); ${ }^{13} \mathrm{C} \mathrm{NMR}\left(\mathrm{CDCl}_{3}, 75 \mathrm{MHz}\right) \delta 149.1,135.8,127.4,125.9,125.8,124.1$; IR v 3416 (w), 3086 (w), 2356 (w), 2149 (s), 1573 (w), 1469 (s), 1395 (m), 1368 (m), $1316(\mathrm{~m}), 1277$ (m), 1233 (m), $1178(\mathrm{~s}), 1083$ (w), $869(\mathrm{~m}), 810(\mathrm{~m})$, $752(\mathrm{~m}), 621(\mathrm{~m}), 597(\mathrm{~m}), 556(\mathrm{~m}), 521(\mathrm{w}), 446(\mathrm{w}) ; \mathrm{MS}(\mathrm{ESI}) 266.1\left(\mathrm{M}\left({ }^{35} \mathrm{Cl}^{35} \mathrm{Cl}\right)-\mathrm{H}\right), 268.1\left(\mathrm{M}\left({ }^{37} \mathrm{Cl}{ }^{35} \mathrm{Cl}\right)-\mathrm{H}\right), 270.1$ $\left(\mathrm{M}\left({ }^{37} \mathrm{Cl}^{37} \mathrm{Cl}\right)-\mathrm{H}\right)$; Anal. calcd for $\mathrm{C}_{6} \mathrm{H}_{3} \mathrm{~N}_{3} \mathrm{O}_{3} \mathrm{SCl}_{2}$ : C, 26.88; H, 1.13; N, 15.67. Found: C, 26.99; H, 1.16; N, 15.64.

5-Metyl-2-methoxy-benzenesulfonyl chloride (209) and 5-Metyl-2-methoxy-benzenesulfonyl azide (46)

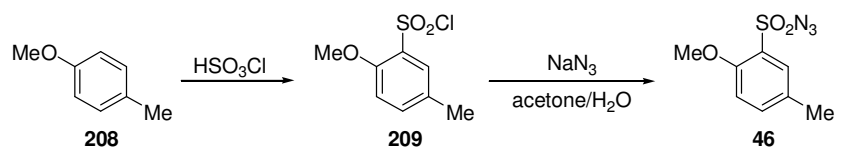

5-Metyl-2-methoxy-benzenesulfonyl chloride (209) ${ }^{38}$

Following a reported procedure, ${ }^{38}$-methoxy toluene $(\mathbf{2 0 8})(10 \mathrm{~mL}, 79 \mathrm{mmol}, 1.0$ equiv) was added dropwise over $20 \mathrm{~min}$ to chlorosulfonic acid ( $26 \mathrm{~mL}, 0.39 \mathrm{~mol}, 5.0$ equiv) at $0{ }^{\circ} \mathrm{C}$ under argon. The reaction mixture was stirred at $0{ }^{\circ} \mathrm{C}$ for $30 \mathrm{~min}$ and at $23{ }^{\circ} \mathrm{C}$ for $5 \mathrm{~h}$, poured carefully onto crushed ice $(80 \mathrm{~g})$ and extracted with $\mathrm{CH}_{2} \mathrm{Cl}_{2}(3 \times 80 \mathrm{~mL})$. The combined organic layers were dried over $\mathrm{Na}_{2} \mathrm{SO}_{4}$ and the solvent was removed under reduced pressure. The isolated product was recrystallized from hexane $/ \mathrm{CH}_{2} \mathrm{Cl}_{2}$ to yield sulfonyl chloride $209(11.2 \mathrm{~g}, 50.8 \mathrm{mmol}, 64 \%)$ as a colorless solid. ${ }^{1} \mathrm{H} \mathrm{NMR}(\mathrm{CDCl} 3,300 \mathrm{MHz}) \delta 7.74$ $(\mathrm{d}, J=1.9 \mathrm{~Hz}, 1 \mathrm{H}, \mathrm{Ar} \mathrm{H}), 7.47(\mathrm{dm}, J=8.5 \mathrm{~Hz}, 1 \mathrm{H}, \mathrm{Ar} \mathrm{H}), 7.02(\mathrm{~d}, J=8.5 \mathrm{~Hz}, 1 \mathrm{H}, \mathrm{Ar} \mathrm{H}), 4.02\left(\mathrm{~s}, 3 \mathrm{H}, \mathrm{OCH}_{3}\right), 2.35(\mathrm{~s}, 3 \mathrm{H}, \mathrm{Ar}$ $\left.\mathrm{CH}_{3}\right) ;{ }^{13} \mathrm{C} \mathrm{NMR}\left(\mathrm{CDCl}_{3}, 75 \mathrm{MHz}\right) \delta 155.2,137.8,131.3,130.0,129.6,113.2,56.6,20.2$; IR v $3122(\mathrm{w}), 2991(\mathrm{w}), 2958(\mathrm{w})$, 2925 (w), 2851 (w), 1608 (w), $1580(w), 1563(w), 1506(\mathrm{~m}), 1459$ (w), $1441(w), 1398(w), 1369(\mathrm{~s}), 1290(\mathrm{~m}), 1264(\mathrm{~m}), 1220$ (w), $1175(\mathrm{~s}), 1064(\mathrm{w}), 1016(\mathrm{~m}), 958(\mathrm{w}), 885(\mathrm{w}), 822(\mathrm{~m}), 745(\mathrm{w}), 716(\mathrm{w}), 692(\mathrm{w}), 587(\mathrm{~s}), 550(\mathrm{~m}), 525(\mathrm{~m}), 445(\mathrm{w})$.

\section{5-Metyl-2-methoxy-benzenesulfonyl azide (46)}

A solution of sodium azide $(4.5 \mathrm{~g}, 69 \mathrm{~mol}, 1.5$ equiv) in water $(30 \mathrm{~mL})$ was added dropwise over 30 min to a solution of sulfonyl chloride (209) $\left(10.1 \mathrm{~g}, 45.7 \mathrm{mmol}, 1.00\right.$ equiv) in acetone $(100 \mathrm{~mL})$ at $0{ }^{\circ} \mathrm{C}$. The reaction was let to warm up to $23{ }^{\circ} \mathrm{C}$ and stirred for $11 \mathrm{~h}$, the acetone was removed under reduced pressure at $25^{\circ} \mathrm{C}$ and the reaction mixture was extracted with AcOEt $(3 \times 50 \mathrm{~mL})$. The combined organic layers were washed with water $(2 \times 50 \mathrm{~mL}), 5 \% \mathrm{Na}_{2} \mathrm{CO}_{3}(2 \times 50 \mathrm{~mL})$, water $(50 \mathrm{~mL})$ and brine $(50 \mathrm{~mL})$, dried over $\mathrm{Na}_{2} \mathrm{SO}_{4}$, the solvent was removed under reduced pressure and the isolated product was dried $2 \mathrm{~h}$ in high vacuo to afford sulfonyl azide $46(10.3 \mathrm{~g}, 45.2 \mathrm{mmol}, 99 \%)$ as a colorless solid. Mp $72-73{ }^{\circ} \mathrm{C} ;{ }^{1} \mathrm{H} \mathrm{NMR}\left(\mathrm{CDCl}_{3}, 300 \mathrm{MHz}\right) \delta 7.68$ $(\mathrm{d}, J=1.9 \mathrm{~Hz}, 1 \mathrm{H}, \mathrm{Ar} \mathrm{H}), 7.41(\mathrm{dm}, J=9.0 \mathrm{~Hz}, 1 \mathrm{H}, \mathrm{Ar} \mathrm{H}), 6.99(\mathrm{~d}, J=9.0 \mathrm{~Hz}, 1 \mathrm{H}, \mathrm{Ar} \mathrm{H}), 3.99\left(\mathrm{~s}, 3 \mathrm{H}, \mathrm{OCH}_{3}\right), 2.33(\mathrm{~s}, 3 \mathrm{H}, \mathrm{Ar}$ $\left.\mathrm{CH}_{3}\right) ;{ }^{13} \mathrm{C} \mathrm{NMR}\left(\mathrm{CDCl}_{3}, 75 \mathrm{MHz}\right) \delta 154.9,136.7,130.2,130.2,127.0,112.2,56.3,20.2 ; \mathrm{IR} v 2955(\mathrm{w}), 2361(\mathrm{w}), 2142(\mathrm{~s}), 1606$ (w), $1585(\mathrm{w}), 1569$ (w), $1498(\mathrm{~s}), 1465(\mathrm{~m}), 1441(\mathrm{w}), 1402(\mathrm{w}), 1360(\mathrm{~s}), 1286(\mathrm{~m}), 1259(\mathrm{~m}), 1221(\mathrm{w}), 1168(\mathrm{~s}), 1068(\mathrm{~m})$, $1019(\mathrm{~m}), 888(\mathrm{~m}), 814(\mathrm{~m}), 749(\mathrm{~s}), 692(\mathrm{~m}), 612(\mathrm{~s}), 593(\mathrm{~s}), 560(\mathrm{~m}), 544(\mathrm{~m}), 477(\mathrm{w}), 459(\mathrm{w}), 431(\mathrm{w})$; MS (ESI) 250.2 $(\mathrm{M}+\mathrm{Na})$; Anal. calcd for $\mathrm{C}_{8} \mathrm{H}_{9} \mathrm{~N}_{3} \mathrm{O}_{3} \mathrm{~S}: \mathrm{C}, 42.28 ; \mathrm{H}, 3.99 ; \mathrm{N}, 18.49$. Found: C, 42.52; H, 4.05; N, 18.29.

2-Chlorosulfonyl-benzoic acid methyl ester (211) and 2-Azidosulfonyl-benzoic acid methyl ester (47)

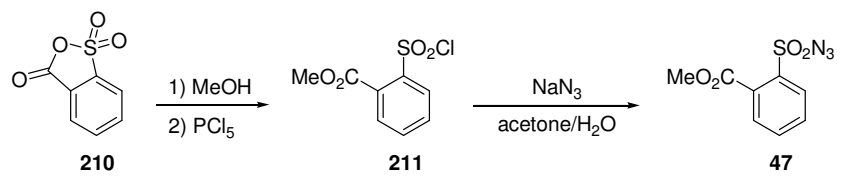

\section{2-Chlorosulfonyl-benzoic acid methyl ester (211) ${ }^{39}$}

Following a known procedure, ${ }^{40}$ a suspension of mixed anhydride $\mathbf{2 1 0}$ (2.0 g, 11 mmol, 1.0 equiv) in methanol (5 mL) was heated to reflux for $1 \mathrm{~h}$. The clear solution was cooled to $23{ }^{\circ} \mathrm{C}$, the solvent was removed under reduced pressure and the isolated sulfonic acid was dried $2 \mathrm{~h}$ in high vacuo. $\mathrm{PCl}_{5}(5.7 \mathrm{~g}, 27 \mathrm{mmol}, 2.5$ equiv) was then added slowly and the viscous mixture was heated to $95{ }^{\circ} \mathrm{C}$ and stirred at this temperature for $2.5 \mathrm{~h}$. The reaction mixture was cooled to $23{ }^{\circ} \mathrm{C}$ and $\mathrm{POCl}_{3}$ was removed under reduced pressure. The residues were suspended in $\mathrm{Et}_{2} \mathrm{O}(30 \mathrm{~mL})$, washed rapidly with ice-water (caution: exothermic reaction, $4 \times 10 \mathrm{~mL}$ ), dried over $\mathrm{Na}_{2} \mathrm{SO}_{4}$, the solvent was removed under reduced pressure and the isolated product was dried $3 \mathrm{~h}$ in high vacuo to yield sulfonyl chloride $211(2.51 \mathrm{~g}, 10.7 \mathrm{mmol}, 97 \%)$ as a viscous oil. ${ }^{1} \mathrm{H} \mathrm{NMR}\left(\mathrm{CDCl}_{3}, 300 \mathrm{MHz}\right) \delta 8.14(\mathrm{dm}, J=8.4$

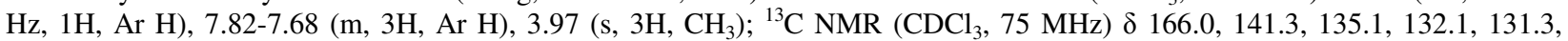
129.9, 128.9, 53.4; IR v 3100 (w), 3012 (w), 2956 (w), 2844 (w), 1792 (w), 1739 (s), 1592 (w), 1571 (w), $1434(\mathrm{~m}), 1378$ (s), 1298 (s), 1260 (s), 1185 (s), 1132 (m), 1119 (m), 1054 (m), 989 (w), 955 (w), 867 (w), 830 (w), 784 (m), 759 (m), 730 (m), 712 (m), $651(\mathrm{~m}), 580(\mathrm{~s}), 559(\mathrm{~s}), 468(\mathrm{w})$.

\section{2-Azidosulfonyl-benzoic acid methyl ester (47)}

A solution of sodium azide $(1.0 \mathrm{~g}, 15 \mathrm{~mol}, 1.5$ equiv) in water $(7.3 \mathrm{~mL})$ was added dropwise over 30 min to a solution of sulfonyl chloride (211) $\left(2.33 \mathrm{~g}, 9.93 \mathrm{mmol}, 1.00\right.$ equiv) in acetone $(24 \mathrm{~mL})$ at $0{ }^{\circ} \mathrm{C}$. The reaction was let to warm up to $23{ }^{\circ} \mathrm{C}$ and stirred for $11 \mathrm{~h}$, the acetone was removed under reduced pressure at $25^{\circ} \mathrm{C}$ and the reaction mixture was extracted with AcOEt $(3 \times 15 \mathrm{~mL})$. The combined organic layers were washed with water $(2 \times 15 \mathrm{~mL}), 5 \% \mathrm{Na}_{2} \mathrm{CO}_{3}(2 \times 15 \mathrm{~mL})$, water $(15 \mathrm{~mL})$ and brine $(15 \mathrm{~mL})$, dried over $\mathrm{Na}_{2} \mathrm{SO}_{4}$, the solvent was removed under reduced pressure and the isolated product was dried $3 \mathrm{~h}$ in high 
vacuo to afford sulfonyl azide $47(2.15 \mathrm{~g}, 8.91 \mathrm{mmol}, 90 \%)$ as a colorless solid. Mp 63-64 ${ }^{\circ} \mathrm{C} ;{ }^{1} \mathrm{H} \mathrm{NMR}\left(\mathrm{CDCl}_{3}, 300 \mathrm{MHz}\right) \delta 8.11$ $(\mathrm{dm}, J=8.4 \mathrm{~Hz}, 1 \mathrm{H}, \mathrm{Ar} \mathrm{H}), 7.78-7.66(\mathrm{~m}, 3 \mathrm{H}, \mathrm{Ar} \mathrm{H}), 3.99\left(\mathrm{~s}, 3 \mathrm{H}, \mathrm{CH}_{3}\right) ;{ }^{13} \mathrm{C} \mathrm{NMR}\left(\mathrm{CDCl}_{3}, 75 \mathrm{MHz}\right) \delta 166.5,137.1,134.5,132.3$, 131.5, 130.2, 130.1, 53.5; IR v 3102 (w), 3012 (w), 2957 (w), 2845 (w), 2364 (w), 2146 (s), 1738 (s), 1626 (w), 1592 (w), 1572 (w), $1476(\mathrm{w}), 1435(\mathrm{~m}), 1370(\mathrm{~s}), 1297(\mathrm{~s}), 1259(\mathrm{~m}), 1175(\mathrm{~s}), 1119(\mathrm{~m}), 1058(\mathrm{~m}), 955(\mathrm{w}), 889(\mathrm{w}), 831(\mathrm{w}), 785(\mathrm{w}), 744(\mathrm{~s})$, $655(\mathrm{~m}), 610$ (s), $568(\mathrm{~s})$; MS (ESI) $264.3(\mathrm{M}+\mathrm{Na})$; Anal. calcd for $\mathrm{C}_{8} \mathrm{H}_{7} \mathrm{~N}_{3} \mathrm{O}_{4} \mathrm{~S}: \mathrm{C}, 39.83 ; \mathrm{H}, 2.92 ; \mathrm{N}, 17.42$. Found: C, 40.04; $\mathrm{H}$, $3.09 ; \mathrm{N}, 17.37$.

\subsection{Synthesis of Alkene Substrates} (3-Methyl-but-3-enyl)-naphthalene (213) ${ }^{41}$

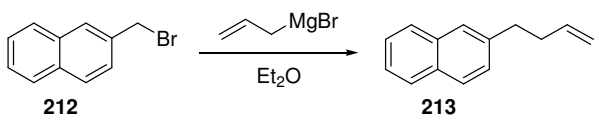

A solution of 2-bromomethyl-naphthalene (212) $\left(5.0 \mathrm{~g}, 23 \mathrm{mmol}, 1.0\right.$ equiv) in dry $\mathrm{Et}_{2} \mathrm{O}(25 \mathrm{~mL})$ was added dropwise over 90 min to a solution of allyl magnesium bromide $\left(1 \mathrm{M}\right.$ in $\mathrm{Et}_{2} \mathrm{O}, 45 \mathrm{~mL}, 45 \mathrm{mmol}, 2.0$ equiv) at $0{ }^{\circ} \mathrm{C}$ under argon. The reaction mixture was warmed to $23{ }^{\circ} \mathrm{C}$ over $14 \mathrm{~h}$, cooled to $0{ }^{\circ} \mathrm{C}$ and quenched with sat. $\mathrm{NH}_{4} \mathrm{Cl}$ solution $(30 \mathrm{~mL})$. After diluting with $\mathrm{H}_{2} \mathrm{O}$ $(30 \mathrm{~mL})$, the reaction mixture was extracted with $\mathrm{Et}_{2} \mathrm{O}(3 \times 50 \mathrm{~mL})$, the combined organic layers were washed with brine $(30 \mathrm{~mL})$, dried over $\mathrm{Na}_{2} \mathrm{SO}_{4}$ and the solvent was removed under reduced pressure. The isolated product was purified by column chromatography (hexane) to afford (3-methyl-but-3-enyl)-naphthalene (213) (3.09 g, $16.9 \mathrm{mmol}, 73 \%)$ as a colorless oil. $\mathrm{R}_{\mathrm{f}}$ (hexane) $0.35 ;{ }^{1} \mathrm{H}$ NMR $\left(\mathrm{CDCl}_{3}, 300 \mathrm{MHz}\right) \delta 7.82-7.76(\mathrm{~m}, 3 \mathrm{H}$, naphthyl $\mathrm{H}), 7.63(\mathrm{~d}, J=0.6 \mathrm{~Hz}, 1 \mathrm{H}$, naphthyl H), 7.48-7.39 (m, $3 \mathrm{H}$, naphthyl H), 7.35 (dd, $J=8.4,1.9 \mathrm{~Hz}, 1 \mathrm{H}$, naphthyl H), 5.95-5.84 (m, 1H, alkene H), 5.11-4.98 (m, 2H, alkene H), 2.91 (t, $J$ $=7.5 \mathrm{~Hz}, 2 \mathrm{H}$, naphthyl- $\left.\mathrm{CH}_{2}\right), 2.51-2.44\left(\mathrm{~m}, 2 \mathrm{H}\right.$, alkene- $\left.\mathrm{CH}_{2}\right) ;{ }^{13} \mathrm{C} \mathrm{NMR}\left(\mathrm{CDCl}_{3}, 75 \mathrm{MHz}\right) \delta 139.3,138.0,133.6,132.0,127.8$, 127.6, 127.4, 127.3, 126.4, 125.8, 125.1, 115.0, 35.5, 35.4; IR v 3053 (m), 3018 (w), 2977 (w), 2925 (m), 2853 (w), 1912 (w), 1821 (w), 1639 (m), 1600 (m), 1508 (m), 1439 (w), 1365 (w), $1332(\mathrm{w}), 1270(\mathrm{w}), 1169(\mathrm{w}), 1143(\mathrm{w}), 1125(\mathrm{w}), 1017(\mathrm{w}), 996$ (w), 959 (w), 911 (s), 889 (w), 853 (s), 815 (s), 745 (s), 648 (w), 617 (w), 475 (s).

But-3-enyloxy-tert-butyl-diphenyl-silane (214) ${ }^{42}$

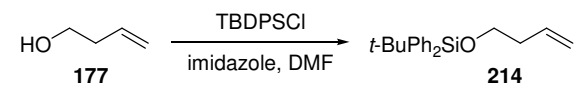

tert-Butyldiphenyl-silyl-chloride ( $3.1 \mathrm{~mL}, 12 \mathrm{mmol}, 1.2$ equiv) was added dropwise to a solution of 3-butenol (177) (0.86 mL, $10 \mathrm{mmol}, 1.0$ equiv) and imidazole $\left(0.82 \mathrm{~g}, 12 \mathrm{mmol}, 1.2\right.$ equiv) in dry DMF $(15 \mathrm{~mL})$ at $23{ }^{\circ} \mathrm{C}$ under argon. After stirring for 20 $\mathrm{h}$, the reaction mixture was quenched with water $(30 \mathrm{~mL})$ and extracted with AcOEt $(3 \times 30 \mathrm{~mL})$. The combined organic layers were washed with water $(4 \times 20 \mathrm{~mL})$ and brine $(20 \mathrm{~mL})$, dried over $\mathrm{Na}_{2} \mathrm{SO}_{4}$ and the solvent was removed under reduced pressure. The isolated product was purified by column chromatography (gradient: hexane, then hexane/ AcOEt 200:1) to afford 214 (3.0 g, $9.8 \mathrm{mmol}, 98 \%)$ as a colorless oil. ${ }^{1} \mathrm{H} \mathrm{NMR}\left(\mathrm{CDCl}_{3}, 300 \mathrm{MHz}\right) \delta 7.70-7.66(\mathrm{~m}, 4 \mathrm{H}, \mathrm{Ar} \mathrm{H}), 7.45-7.35(\mathrm{~m}, 6 \mathrm{H}, \mathrm{Ar} \mathrm{H}), 5.89-5.77$ $\left(\mathrm{m}, 1 \mathrm{H}\right.$, alkene-H), 5.10-4.99 (m, 2H, alkene-H), $3.72\left(\mathrm{t}, J=6.5 \mathrm{~Hz}, 2 \mathrm{H}, \mathrm{CH}_{2} \mathrm{O}\right), 2.36-2.28(\mathrm{~m}, 2 \mathrm{H}$, alkene-CH$), 1.06(\mathrm{~s}, 9 \mathrm{H}$, $\left.\mathrm{CH}_{3}\right) ;{ }^{13} \mathrm{C} \mathrm{NMR}\left(\mathrm{CDCl}_{3}, 75 \mathrm{MHz}\right) \delta 135.6,135.4,134.0,129.5,127.6,116.4,63.5,37.2,26.8,19.2 ; \mathrm{IR} v 3071(\mathrm{~m}), 3050(\mathrm{w})$, 3013 (w), 2958 (m), 2930 (m), $2894(\mathrm{~m}), 2857(\mathrm{~m}), 1958(\mathrm{w}), 1887(\mathrm{w}), 1822(\mathrm{w}), 1641(\mathrm{w}), 1589(\mathrm{w}), 1472(\mathrm{~m}), 1462(\mathrm{w}), 1428$ (m), 1389 (w), 1361 (w), 1329 (w), 1305 (w), 1263 (w), 1227 (w), 1188 (w), $1111(\mathrm{~s}), 1007$ (w), 998 (w), 988 (w), 912 (m), 823 (m), 737 (m), 701 (s), 689 (m), 613 (m), 506 (s), 491 (m).

Prop-2-enyloxy-tert-butyl-diphenyl-silane (215) $)^{43}$

$$
\underset{119}{\stackrel{\text { TBDPSCl }}{\text { imidazole, DMF }}} t \text {-BuPh }_{2} \mathrm{SiO}_{215}
$$

tert-Butyldiphenyl-silyl-chloride $(8.4 \mathrm{~mL}, 32 \mathrm{mmol}, 1.1$ equiv) was added dropwise to a solution of allyl alcohol (119) $(2.0 \mathrm{~mL}$, $29 \mathrm{mmol}, 1.0$ equiv) and imidazole $\left(2.4 \mathrm{~g}, 35 \mathrm{mmol}, 1.2\right.$ equiv) in dry DMF $(25 \mathrm{~mL})$ at $23^{\circ} \mathrm{C}$ under argon. After stirring for $2 \mathrm{~h}$, the reaction mixture was quenched with water $(60 \mathrm{~mL})$ and extracted with $\mathrm{Et}_{2} \mathrm{O}(3 \times 60 \mathrm{~mL})$. The combined organic layers were washed with water $(4 \times 40 \mathrm{~mL})$ and brine $(40 \mathrm{~mL})$, dried over $\mathrm{Na}_{2} \mathrm{SO}_{4}$ and the solvent was removed under reduced pressure. The isolated product was purified by column chromatography (hexane/AcOEt 50:1) to afford 215 (8.91 g, 30.0 mmol, 100\%) as a colorless oil. ${ }^{1} \mathrm{H}$ NMR $\left(\mathrm{CDCl}_{3}, 300 \mathrm{MHz}\right) \delta$ 7.71-7.67 (m, 4H, Ar H), 7.46-7.35 (m, 6H, Ar H), 5.98-5.87 (m, 1H, alkene-H), 5.42-5.34 (m, 1H, alkene-H), 5.15-5.09 (m, 1H, alkene-H), 4.22-4.20 (m, 2H, $\left.\mathrm{CH}_{2} \mathrm{O}\right), 1.07\left(\mathrm{~s}, 9 \mathrm{H}, \mathrm{CH}_{3}\right) ;{ }^{13} \mathrm{C} \mathrm{NMR}\left(\mathrm{CDCl}_{3}, 75\right.$ MHz) $\delta 136.9,135.4,133.6,129.5,127.5,113.8,64.6,26.9,19.4$; IR v 3070 (m), 3050 (w), 3013 (w), 2998 (w), 2958 (m), 2930 $(\mathrm{m}), 2891(\mathrm{~m}), 2857(\mathrm{~s}), 1959(\mathrm{w}), 1888(\mathrm{w}), 1824(\mathrm{w}), 1771(\mathrm{w}), 1646(\mathrm{w}), 1589(\mathrm{w}), 1487(\mathrm{w}), 1472(\mathrm{~m}), 1462(\mathrm{~m}), 1427(\mathrm{~s})$, 1402 (w), 1398 (w), 1378 (w), $1361(\mathrm{w}), 1306(\mathrm{w}), 1288$ (w), $1261(\mathrm{w}), 1188(\mathrm{w}), 1134$ (m), $1112(\mathrm{~s}), 1079(\mathrm{~m}), 1035$ (m), 1008 (m), $998(\mathrm{~m}), 916$ (m), $824(\mathrm{~m}), 811(\mathrm{~m}), 740(\mathrm{~m}), 701(\mathrm{~s}), 620(\mathrm{~m}), 616(\mathrm{~m}), 564(\mathrm{w}), 505(\mathrm{~s}), 490(\mathrm{~m})$.

Pent-4-enoic acid benzyl ester $(217)^{44}$

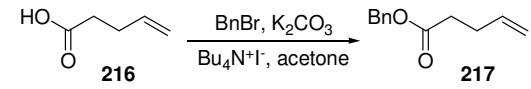

Benzyl bromide (1.3 mL, $12 \mathrm{mmol}, 1.2$ equiv) was added to a solution of 4-pentanoic acid (216) (1.0 mL, $9.8 \mathrm{mmol}, 1.0$ equiv) in acetone $(20 \mathrm{~mL})$ at $23{ }^{\circ} \mathrm{C}$ under argon. Potassium carbonate $(6.8 \mathrm{~g}, 49 \mathrm{mmol}, 5.0$ equiv) and tetrabutylammonium iodide $(0.24$ $\mathrm{g}, 1.0 \mathrm{mmol}, 0.10$ equiv) were added and the resulting colorless suspension was stirred for $15 \mathrm{~h}$, filtered and the solvent was 
removed under reduced pressure. The reaction mixture was then dissolved in AcOEt (40 mL), washed with $2 \mathrm{M} \mathrm{HCl}(30 \mathrm{~mL})$, sat. $\mathrm{NaHCO}_{3}(30 \mathrm{~mL})$ and brine $(30 \mathrm{~mL})$, dried over $\mathrm{Na}_{2} \mathrm{SO}_{4}$ and the solvent was removed under reduced pressure. The isolated product was purified by column chromatography (AcOEt/hexane 30:1) to afford $217(1.65 \mathrm{~g}, 8.67 \mathrm{mmol}, 88 \%)$ as a colorless oil. ${ }^{1} \mathrm{H}$ NMR $\left(\mathrm{CDCl}_{3}, 300 \mathrm{MHz}\right) \delta$ 7.39-7.33 (m, 5H, Ar H), 5.91-5.78 (m, 1H, alkene-H), $5.14\left(\mathrm{~s}, 2 \mathrm{H}, \mathrm{PhCH}_{2}\right), 5.14-5.00(\mathrm{~m}, 2 \mathrm{H}$, alkene-H), 2.52-2.39 (m, 4H, $\mathrm{CH}_{2} \mathrm{CO}$ and alkene- $\left.\mathrm{CH}_{2}\right) ;{ }^{13} \mathrm{C} \mathrm{NMR}\left(\mathrm{CDCl}_{3}, 75 \mathrm{MHz}\right) \delta 172.6,136.4,135.8,128.4,128.0,115.4$, 66.1, 33.5, 28.9; IR v 3067 (w), 3034 (w), 2979 (w), 2959 (w), 1738 (s), 1642 (w), 1608 (w), 1587 (w), 1498 (w), 1455 (m), 1418 (w), $1381(\mathrm{~m}), 1350(\mathrm{~m}), 1257(\mathrm{~m}), 1213(\mathrm{~m}), 1165(\mathrm{~s}), 1115(\mathrm{w}), 1002(\mathrm{~m}), 916(\mathrm{~m}), 844(\mathrm{w}), 825(\mathrm{w}), 786(\mathrm{w}), 751(\mathrm{~m}), 698(\mathrm{~s})$, $577(\mathrm{w}), 500(\mathrm{w}), 450(\mathrm{w})$.

\section{1-Phenyl-pent-4-en-1-one (219) ${ }^{45}$}

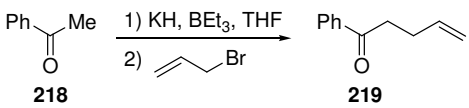

A solution of acetophenone $(\mathbf{2 1 8})(2.6 \mathrm{~mL}, 22 \mathrm{mmol}, 1.0$ equiv) in dry THF ( $8 \mathrm{~mL})$ was added dropwise over $10 \mathrm{~min}$ to a suspension of potassium hydride $\left(1.1 \mathrm{~g}, 27 \mathrm{mmol}, 1.2\right.$ equiv) in dry THF $(45 \mathrm{~mL})$ at $0{ }^{\circ} \mathrm{C}$ under argon. The yellow suspension was stirred at $23{ }^{\circ} \mathrm{C}$ for $30 \mathrm{~min}$ and $\mathrm{BEt}_{3}\left(4.0 \mathrm{~mL}, 28 \mathrm{mmol}, 1.2\right.$ equiv) was added dropwise at $15{ }^{\circ} \mathrm{C}$ over $15 \mathrm{~min}$. After stirring the resulting clear yellow solution at $23{ }^{\circ} \mathrm{C}$ for $15 \mathrm{~min}$, allyl bromide (freshly distilled, $2.9 \mathrm{~mL}, 34 \mathrm{mmol}, 1.5$ equiv) was added dropwise over $10 \mathrm{~min}$ and the resulting suspension was stirred for $4 \mathrm{~h}$ at $23{ }^{\circ} \mathrm{C}$ and quenched with a $1: 1 \mathrm{mixture}$ of $30 \% \mathrm{NaOH}$ and $30 \% \mathrm{H}_{2} \mathrm{O}_{2}(24 \mathrm{~mL})$ at $0^{\circ} \mathrm{C}$ over 15 min. The reaction mixture was then diluted with $\mathrm{H}_{2} \mathrm{O}(40 \mathrm{~mL})$, the layers were separated and the organic layer diluted with $\mathrm{Et}_{2} \mathrm{O}(100 \mathrm{~mL})$ and washed with water $(2 \times 50 \mathrm{~mL})$. The combined water layers were extracted with $\mathrm{CH}_{2} \mathrm{Cl}_{2}(2 \times 50 \mathrm{~mL})$ and the combined organic layers were dried over $\mathrm{Na}_{2} \mathrm{SO}_{4}$ and the solvents were removed under reduced pressure. The isolated product was purified by column chromatography (AcOEt/hexane 1:30) to afford 219 (2.85 g, $17.8 \mathrm{mmol}$, $80 \%)$ as a colorless oil. ${ }^{1} \mathrm{H}$ NMR $\left(\mathrm{CDCl}_{3}, 300 \mathrm{MHz}\right) \delta 7.97(\mathrm{~d}, J=7.2 \mathrm{~Hz}, 2 \mathrm{H}, \mathrm{Ar} \mathrm{H}), 7.59-7.43(\mathrm{~m}, 3 \mathrm{H}, \mathrm{Ar} \mathrm{H}), 5.98-5.84(\mathrm{~m}, 1 \mathrm{H}$, alkene-H), 5.13-4.99 (m, 2H, alkene-H), 3.11-3.06 (m, 2H, $\left.\mathrm{CH}_{2} \mathrm{CO}\right), 2.54-2.46\left(\mathrm{~m}, 2 \mathrm{H}\right.$, alkene- $\left.\mathrm{CH}_{2}\right) ;{ }^{13} \mathrm{C} \mathrm{NMR}\left(\mathrm{CDCl}{ }_{3}, 75 \mathrm{MHz}\right)$ $\delta$ 199.1, 137.1, 136.8, 132.9, 128.4, 127.9, 115.2, 37.8, 28.2; IR v 3067 (w), 2977 (w), $2922(w), 2855$ (w), 1820 (w), 1687 (s), 1641 (w), 1597 (w), 1580 (w), 1448 (m), $1411(w), 1361(w), 1268$ (w), 1207 (m), $1180(w), 1159$ (w), $1102(w), 1076(w), 1001$ (w), $971(\mathrm{w}), 913(\mathrm{~m}), 744(\mathrm{~m}), 690(\mathrm{~m}), 656(\mathrm{w}), 567(\mathrm{w})$.

\section{(3-Methyl-but-3-enyl)-benzene (221 $)^{46}$}

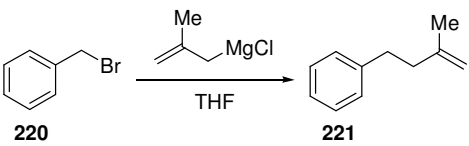

Methallyl chloride (5.3 mL, $54 \mathrm{mmol}, 2.5$ equiv) was added slowly (10 min) to a suspension of magnesium turnings (1.3 g, 55 mmol, 2.5 equiv) in dry THF $(40 \mathrm{~mL})$ at $15{ }^{\circ} \mathrm{C}$. The resulting suspension was stirred at $23{ }^{\circ} \mathrm{C}$ for $2 \mathrm{~h}$ and refluxed for $2 \mathrm{~h}$. After cooling to $23{ }^{\circ} \mathrm{C}$, the suspension was decanted and transferred via canula to a $100 \mathrm{~mL}-S c h l e n k$ flask. The solution was titrated (quenched with exact amount of $0.2 \mathrm{M} \mathrm{HCl}$, neutralisation of excess $\mathrm{HCl}$ with $0.2 \mathrm{M} \mathrm{NaOH}$ over phenolphthalein) and appeared to be $0.6 \mathrm{M}$ (24 mmol Grignard reagent, 1.1 equiv). Benzyl bromide (220) $\left(2.6 \mathrm{~mL}, 22 \mathrm{mmol}, 1.0\right.$ equiv) was then added at $0{ }^{\circ} \mathrm{C}$ over $3 \mathrm{~h}$ via syringe pump and the reaction mixture was refluxed for $3 \mathrm{~h}$, cooled to $0{ }^{\circ} \mathrm{C}$ and quenched with sat. $\mathrm{NH}_{4} \mathrm{Cl}(20 \mathrm{~mL})$. After diluting with $\mathrm{H}_{2} \mathrm{O}(20 \mathrm{~mL})$, the reaction mixture was extracted with $\mathrm{Et}_{2} \mathrm{O}(3 \times 40 \mathrm{~mL})$, the combined organic layers were washed with brine $(20 \mathrm{~mL})$, dried over $\mathrm{Na}_{2} \mathrm{SO}_{4}$ and the solvent was removed under reduced pressure $\left(p=50 \mathrm{mbar}, T=40{ }^{\circ} \mathrm{C}\right)$. The isolated product was purified by column chromatography (pentane) to afford (3-methyl-but-3-enyl)-benzene (221) (2.22 g, $15.2 \mathrm{mmol}, 69 \%)$ as a colorless liquid. $\mathrm{R}_{\mathrm{f}}$ (hexane) $0.60 ;{ }^{1} \mathrm{H} \mathrm{NMR}\left(\mathrm{CDCl}_{3}, 300 \mathrm{MHz}\right) \delta 7.34-7.18(\mathrm{~m}, 5 \mathrm{H}, \mathrm{Ar} \mathrm{H}), 4.78-4.75(\mathrm{~m}$, $2 \mathrm{H}$, alkene $\mathrm{H}), 2.82-2.76\left(\mathrm{~m}, 2 \mathrm{H}, \mathrm{PhCH}_{2}\right), 2.38-2.33\left(\mathrm{~m} .2 \mathrm{H}\right.$, alkene- $\left.\mathrm{CH}_{2}\right), 1.81\left(\mathrm{~s}, 3 \mathrm{H}, \mathrm{CH}_{3}\right) ;{ }^{13} \mathrm{C} \mathrm{NMR}\left(\mathrm{CDCl}_{3}, 75 \mathrm{MHz}\right) \delta$ 145.2, 142.1, 128.2, 125.6, 110.1, 39.7, 34.3, 22.7; IR v 3064 (w), 3027 (m), 2968 (m), $2936(\mathrm{~m}), 2857$ (w), $1800(\mathrm{w}), 1649$ (m), 1604 (w), 1495 (m), 1453 (s), 1374 (w), 1078 (w), 1031 (w), 887 (s), 745 (m), 697 (s), 574 (w), 520 (w).

tert-Butyl-(2-methyl-allyloxy)-diphenyl-silane $(\mathbf{2 2 3})^{47}$

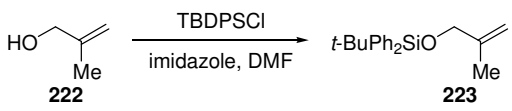

tert-Butyldiphenyl-silyl-chloride $(3.1 \mathrm{~mL}, 12 \mathrm{mmol}, 1.2$ equiv) was added dropwise to a solution of methallyl alcohol (222) ( $0.85 \mathrm{~mL}, 10 \mathrm{mmol}, 1.0$ equiv) and imidazole $\left(0.82 \mathrm{~g}, 12 \mathrm{mmol}, 1.2\right.$ equiv) in dry DMF $(15 \mathrm{~mL})$ at $23{ }^{\circ} \mathrm{C}$ under argon. After stirring for $15 \mathrm{~h}$, the reaction mixture was quenched with water $(30 \mathrm{~mL})$ and extracted with AcOEt $(3 \times 30 \mathrm{~mL})$. The combined organic layers were washed with water $(4 \times 20 \mathrm{~mL})$ and brine $(20 \mathrm{~mL})$, dried over $\mathrm{Na}_{2} \mathrm{SO}_{4}$ and the solvent was removed under reduced pressure. The isolated product was purified by column chromatography (pentane/Et ${ }_{2} \mathrm{O} 100: 1$ ) to afford 223 (3.1 g, 9.9 mmol, 99\%) as a colorless oil. ${ }^{1} \mathrm{H}$ NMR $\left(\mathrm{CDCl}_{3}, 300 \mathrm{MHz}\right) \delta 7.71-7.68(\mathrm{~m}, 4 \mathrm{H}, \mathrm{Ar} \mathrm{H}), 7.43-7.35(\mathrm{~m}, 6 \mathrm{H}, \mathrm{Ar} \mathrm{H}), 5.13(\mathrm{~s}, 1 \mathrm{H}$, alkene-H), $4.86\left(\mathrm{~s}, 1 \mathrm{H}\right.$, alkene-H), $4.07\left(\mathrm{~s}, 2 \mathrm{H}, \mathrm{CH}_{2} \mathrm{O}\right), 1.69$ (s, 3H, alkene- $\left.\mathrm{CH}_{3}\right), 1.07\left(\mathrm{~s}, 9 \mathrm{H}, \mathrm{CCH}_{3}\right) ;{ }^{13} \mathrm{C} \mathrm{NMR}\left(\mathrm{CDCl}_{3}, 75 \mathrm{MHz}\right)$ $\delta$ 144.0, 135.4, 133.6, 129.5, 127.6, 109.1, 67.3, 26.9, 19.4, 19.1; IR v $3071(\mathrm{w}), 3050$ (w), $2998(\mathrm{w}), 2959(\mathrm{~m}), 2931(\mathrm{~m}), 2893$ (m), 2857 (m), 1959 (w), 1889 (w), 1822 (w), 1659 (w), 1589 (w), $1486(\mathrm{w}), 1472$ (m), $1462(\mathrm{w}), 1428$ (m), $1390(\mathrm{w}), 1361(\mathrm{w})$, $1306(\mathrm{w}), 1262(\mathrm{w}), 1188$ (w), $1112(\mathrm{~s}), 1029(\mathrm{w}), 1007$ (w), 998 (w), 938 (w), $896(\mathrm{~m}), 826(\mathrm{~m}), 740(\mathrm{~m}), 701(\mathrm{~s}), 614(\mathrm{~m}), 504$ (s), $490(\mathrm{~m}), 436(\mathrm{w})$.

tert-Butyl-(3-methyl-but-2-enyloxy)-diphenyl-silane $(\mathbf{2 2 5})^{48}$ 


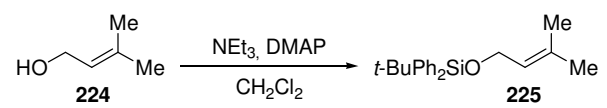

tert-Butyldiphenyl-silyl-chloride ( $2.1 \mathrm{~mL}, 22 \mathrm{mmol}, 1.2$ equiv) was added dropwise to a solution of 3-methy-2-butenol (224) $\left(1.0 \mathrm{~mL}, 10 \mathrm{mmol}, 1.0\right.$ equiv) in dry $\mathrm{CH}_{2} \mathrm{Cl}_{2}(17 \mathrm{~mL})$ at $23{ }^{\circ} \mathrm{C}$ under argon. Triethylamine (freshly distilled, $1.7 \mathrm{~mL}, 12 \mathrm{mmol}, 1.2$ equiv) and 4-dimethylamino-pyridine ( $48 \mathrm{mg}, 0.39 \mathrm{mmol}, 0.039$ equiv) were added. After stirring $48 \mathrm{~h}$ at $23{ }^{\circ} \mathrm{C}$ the reaction mixture was diluted with $\mathrm{CH}_{2} \mathrm{Cl}_{2}$ and washed with water $(2 \times 50 \mathrm{~mL})$, dried over $\mathrm{Na}_{2} \mathrm{SO}_{4}$ and the solvent was removed under reduced pressure. The isolated product was purified by column chromatography (AcOEt/hexane 1:100) to afford 225 (2.54 g, 7.83 mmol, $78 \%)$ as a colorless oil. ${ }^{1} \mathrm{H}$ NMR $\left(\mathrm{CDCl}_{3}, 300 \mathrm{MHz}\right) \delta$ 7.75-7.71 (m, 4H, Ar H), 7.47-7.37 (m, 6H, Ar H), 5.44-5.38 (m, $1 \mathrm{H}$, alkene-H), $4.23\left(\mathrm{~d}, J=6.5 \mathrm{~Hz}, 2 \mathrm{H}, \mathrm{CH}_{2} \mathrm{O}\right), 1.72\left(\mathrm{~m}, 3 \mathrm{H}\right.$, alkene- $\left.\mathrm{CH}_{3}\right), 1.48\left(\mathrm{~s}, 3 \mathrm{H}\right.$, alkene- $\left.\mathrm{CH}_{3}\right), 1.08\left(\mathrm{~s}, 9 \mathrm{H}, \mathrm{CCH}_{3}\right) ;{ }^{13} \mathrm{C}$ NMR $\left(\mathrm{CDCl}_{3}, 75 \mathrm{MHz}\right) \delta 135.6,134.0,133.8,129.5,127.6,124.2,61.1,26.8,25.7,19.2,17.9 ;$ IR $v 3070$ (w), 3049 (w), 2960 (m), 2930 (m), $2891(\mathrm{~m}), 2857(\mathrm{~m}), 2104(\mathrm{w}), 1958(\mathrm{w}), 1888(\mathrm{w}), 1824(\mathrm{w}), 1771(\mathrm{w}), 1722(\mathrm{w}), 1675(\mathrm{w}), 1589(\mathrm{w}), 1487(\mathrm{w})$, $1472(\mathrm{~m}), 1462(\mathrm{w}), 1446(\mathrm{w}), 1428(\mathrm{~m}), 1380(\mathrm{w}), 1361(\mathrm{w}), 1329(\mathrm{w}), 1261(\mathrm{w}), 1189(\mathrm{w}), 1112(\mathrm{~s}), 1062(\mathrm{~s}), 1028(\mathrm{~m}), 1007$ (w), $998(\mathrm{w}), 938(\mathrm{w}), 913(\mathrm{w}), 855(\mathrm{w}), 823(\mathrm{~m}), 774(\mathrm{w}), 739(\mathrm{~m}), 701(\mathrm{~s}), 689(\mathrm{~m}), 613(\mathrm{~m}), 504(\mathrm{~s}), 440(\mathrm{w})$.

\section{((E)-3-Methyl-pent-3-enyl)-benzene $(227)^{49}$}

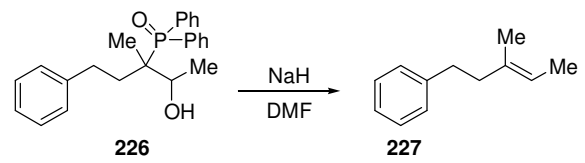

A suspension of 3-(diphenyl-phosphinoyl)-3-methyl-5-phenyl-pentan-2-ol (226) (666 mg, 1.76 mmol, 1.00 equiv, diastereomerically pure, synthesized following a published procedure $)^{49}$ in dry DMF $(30 \mathrm{~mL})$ was added to a suspension of sodium hydride (45 mg, $1.8 \mathrm{mmol}, 1.0$ equiv) in dry DMF $(15 \mathrm{~mL})$. After heating the resulting suspension at $50{ }^{\circ} \mathrm{C}$ for $1 \mathrm{~h}$, the reaction mixture was cooled to $0{ }^{\circ} \mathrm{C}$, quenched with water $(50 \mathrm{~mL})$, diluted with water $(20 \mathrm{~mL})$ and brine $(70 \mathrm{~mL})$ and extracted with $\mathrm{Et}_{2} \mathrm{O}(3 \times 100 \mathrm{~mL})$. The combined organic layers were washed with water $(4 \times 50 \mathrm{~mL})$ and brine $(50 \mathrm{~mL}), \mathrm{dried}^{\circ} \mathrm{over} \mathrm{Na}_{2} \mathrm{SO}_{4}$ and the solvent was removed under reduced pressure $\left(p=100 \mathrm{mbar}, T=40{ }^{\circ} \mathrm{C}\right)$. The isolated product was purified by column chromatography (pentane) to afford $227(101 \mathrm{mg}, 0.631 \mathrm{mmol}, 36 \%)$ as a colorless liquid. ${ }^{1} \mathrm{H} \mathrm{NMR}\left(\mathrm{CDCl}_{3}, 300 \mathrm{MHz}\right) \delta 7.31-$ 7.25 (m, 2H, Ar H), 7.20-7.15 (m, 3H, Ar H), 5.27-5.20 (m, 1H, alkene-H), 2.73-2.68 (m, 2H, $\left.\mathrm{PhCH}_{2}\right), 2.30-2.25(\mathrm{~m}, 2 \mathrm{H}$, alkene$\left.\mathrm{CH}_{2}\right), 1.66\left(\mathrm{~s}, 3 \mathrm{H}, \mathrm{CH}_{3}\right), 1.58\left(\mathrm{~d}, J=6.5 \mathrm{~Hz}, 3 \mathrm{H}, \mathrm{CH}_{3}\right) ;{ }^{13} \mathrm{C} \mathrm{NMR}\left(\mathrm{CDCl}_{3}, 75 \mathrm{MHz}\right) \delta 142.4,135.2,128.3,128.1,125.5,118.7$, 41.7, 34.9, 15.9, 13.5; IR v 3026 (w), 2922 (m), 2858 (w), 1602 (w), $1496(w), 1453$ (w), $1380(w), 1030(w), 913(\mathrm{~m}), 807$ (w), $743(\mathrm{~s}), 697(\mathrm{~s}), 516(\mathrm{w}), 451(\mathrm{w})$.

(2-Methyl-allyloxymethyl)-benzene (228) ${ }^{50}$

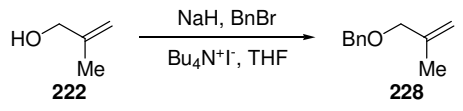

A solution of methallyl alcohol (222) $(2.0 \mathrm{~mL}, 24 \mathrm{mmol}, 1.0$ equiv) in THF ( $8 \mathrm{~mL}$ ) was added dropwise to a suspension of NaH $\left(0.63 \mathrm{~g}, 25 \mathrm{mmol}, 1.0\right.$ equiv) in THF $(8 \mathrm{~mL})$ at $23{ }^{\circ} \mathrm{C}$ under argon. Ater $1 \mathrm{~h}$, a solution of benzyl bromide $(2.8 \mathrm{~mL}, 24 \mathrm{mmol}, 1$ equiv) in THF $(8 \mathrm{~mL})$ and tetrabutylammonium iodide $(0.24 \mathrm{~g}, 0.65 \mathrm{mmol}, 0.027$ equiv) were added slowly and the reaction mixture was heated to $40^{\circ} \mathrm{C}$ for $3.5 \mathrm{~h}$. After cooling to $23{ }^{\circ} \mathrm{C}$, the reaction mixture was quenched with water $(20 \mathrm{~mL})$ and extracted with $\mathrm{Et}_{2} \mathrm{O}(3 \times 50 \mathrm{~mL})$. The combined organic layers were washed with water $(30 \mathrm{~mL})$ and brine $(30 \mathrm{~mL})$, dried over $\mathrm{Na}_{2} \mathrm{SO}_{4}$ and the solvent was removed under reduced pressure. The isolated product was distilled $\left(p=0.5 \mathrm{mbar}, b p=45^{\circ} \mathrm{C}\right)$ to afford 228 (3.58 g, $22.1 \mathrm{mmol}, 92 \%)$ as a colorless liquid. ${ }^{1} \mathrm{H} \mathrm{NMR}\left(\mathrm{CDCl}_{3}, 300 \mathrm{MHz}\right) \delta 7.46-7.33(\mathrm{~m}, 5 \mathrm{H}, \mathrm{Ar} \mathrm{H}), 5.11(\mathrm{~m}, 1 \mathrm{H}$, alkene $\mathrm{H}), 5.02(\mathrm{~m}, 1 \mathrm{H}$, alkene $\mathrm{H}), 4.58\left(\mathrm{~s}, 2 \mathrm{H}, \mathrm{CH}_{2} \mathrm{O}\right), 4.02\left(\mathrm{~s}, 2 \mathrm{H}, \mathrm{CH}_{2} \mathrm{O}\right), 1.87\left(\mathrm{~d}, \mathrm{~J}=0.6 \mathrm{~Hz}, 3 \mathrm{H}\right.$, alkene $\left.\mathrm{CH}_{3}\right) ;{ }^{13} \mathrm{C} \mathrm{NMR}$ $\left(\mathrm{CDCl}_{3}, 75 \mathrm{MHz}\right) \delta 141.9,138.2,128.1,127.4,127.3,112.1,74.0,71.7,19.5$; IR v $3066(\mathrm{w}), 3031(\mathrm{w}), 2974(\mathrm{w}), 2915(\mathrm{~m}), 2854$ (m), 1950 (w), 1870 (w), 1809 (w), 1656 (w), 1605 (w), 1496 (m), 1453 (s), 1363 (m), 1350 (m), 1310 (w), 1254 (w), 1203 (w), 1099 (s), 1075 (s), 1028 (m), 985 (w), 944 (w), 901 (s), 819 (w), 736 (s), 697 (s), 610 (w), 553 (w), 467 (w).

\subsection{The Hydroazidation of Olefins}

\subsubsection{General Procedures}

General Procedure for the Co-Catalyzed Hydroazidation of Olefins with Phenylsilane (GP5)

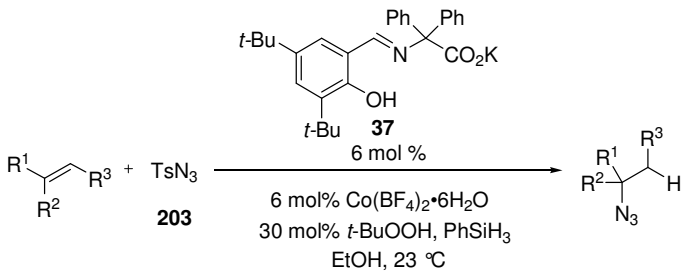


$\mathrm{Co}\left(\mathrm{BF}_{4}\right)_{2} \bullet 6 \mathrm{H}_{2} \mathrm{O}(10 \mathrm{mg}, 0.030 \mathrm{mmol}, 0.060$ equiv) and ligand 37 (14 mg, $0.030 \mathrm{mmol}, 0.060$ equiv) were dissolved in ethanol $(2.5 \mathrm{~mL})$ at $23{ }^{\circ} \mathrm{C}$ under argon. After $10 \mathrm{~min}$, the alkene $(0.50 \mathrm{mmol}, 1.0$ equiv) was added to the homogenous orange solution, followed by tosyl azide $(\mathbf{2 0 3})(0.23 \mathrm{~mL}, 1.5 \mathrm{mmol}, 3.0$ equiv) and tert-butyl hydroperoxide $(5.5 \mathrm{M}$ in decane, $25 \mu \mathrm{L}, 0.14 \mathrm{mmol}$, 0.28 equiv). After $5 \mathrm{~min}$, phenylsilane $(0.10 \mathrm{~mL}, 0.80 \mathrm{mmol}, 1.6$ equiv) was added dropwise. The resulting brown solution was stirred at $23{ }^{\circ} \mathrm{C}$ and the reaction monitored by TLC (AcOEt/hexane 1:40). After completion the reaction was quenched with $\mathrm{H}_{2} \mathrm{O}$ $(2 \mathrm{~mL})$. Sat. $\mathrm{NaHCO}_{3}(2 \mathrm{~mL})$ and brine $(5 \mathrm{~mL})$ were added and the reaction mixture was extracted with AcOEt $(3 \times 10 \mathrm{~mL})$. The combined organic layers were dried over $\mathrm{Na}_{2} \mathrm{SO}_{4}$, filtered and the solvents were removed under reduced pressure $(p=50 \mathrm{mbar}, T$ $=40^{\circ} \mathrm{C}$ ). The isolated product was purified by column chromatography to afford the desired alkyl azide.

General Procedure for the Co-Catalyzed Hydroazidation of Olefins with TMDSO (260) (GP6)

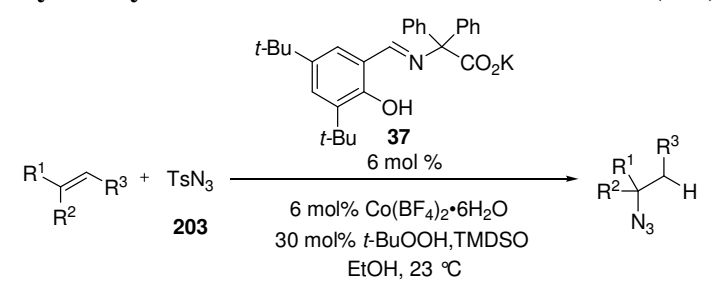

$\mathrm{Co}\left(\mathrm{BF}_{4}\right)_{2} \bullet 6 \mathrm{H}_{2} \mathrm{O}$ (10 mg, $0.030 \mathrm{mmol}, 0.060$ equiv) and ligand 37 (14 mg, $0.030 \mathrm{mmol}, 0.060$ equiv) were dissolved in ethanol $(2.5 \mathrm{~mL})$ at $23{ }^{\circ} \mathrm{C}$ under argon. After $10 \mathrm{~min}$, the alkene $(0.50 \mathrm{mmol}, 1.0$ equiv) was added to the homogenous orange solution, followed by tosyl azide $(\mathbf{2 0 3})(0.23 \mathrm{~mL}, 1.5 \mathrm{mmol}, 3.0$ equiv) and tert-butyl hydroperoxide $(5.5 \mathrm{M}$ in decane, $25 \mu \mathrm{L}, 0.14 \mathrm{mmol}$, 0.28 equiv). After $5 \mathrm{~min}$, TMDSO ( $0.18 \mathrm{~mL}, 1.0 \mathrm{mmol}, 2.0$ equiv, for some substrates: $0.26 \mathrm{~mL}, 1.5 \mathrm{mmol}, 3.0$ equiv) was added dropwise. The resulting dark brown-green solution was stirred at $23{ }^{\circ} \mathrm{C}$ and the reaction monitored by TLC (AcOEt/hexane 1:40). After completion the reaction was quenched with $\mathrm{H}_{2} \mathrm{O}(2 \mathrm{~mL})$. Sat. $\mathrm{NaHCO}_{3}(2 \mathrm{~mL})$ and brine $(5 \mathrm{~mL})$ were added and the reaction mixture was extracted with AcOEt $(3 \times 10 \mathrm{~mL})$. The combined organic layers were dried over $\mathrm{Na}_{2} \mathrm{SO}_{4}$, filtered and the solvents were removed under reduced pressure $\left(p=50 \mathrm{mbar}, T=40{ }^{\circ} \mathrm{C}\right)$. The isolated product was purified by column chromatography to afford the desired alkyl azide.

General Procedure for the Co-Catalyzed Hydroazidation of Olefins with TMDSO and sulfonylazide 52 (GP7)

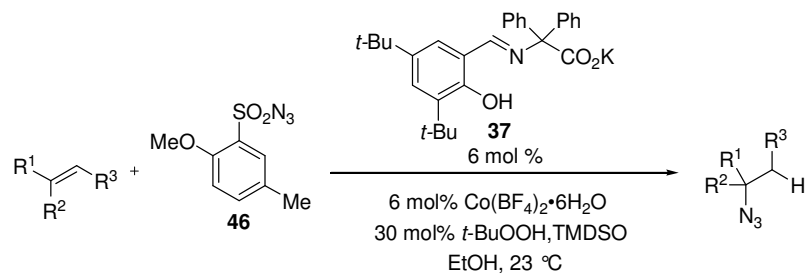

$\mathrm{Co}\left(\mathrm{BF}_{4}\right)_{2} \bullet 6 \mathrm{H}_{2} \mathrm{O}(10 \mathrm{mg}, 0.030 \mathrm{mmol}, 0.060$ equiv) and ligand 37 (14 mg, $0.030 \mathrm{mmol}, 0.060$ equiv) were dissolved in ethanol $(2.5 \mathrm{~mL})$ at $23{ }^{\circ} \mathrm{C}$ under argon. After $10 \mathrm{~min}$, the alkene $(0.50 \mathrm{mmol}, 1.0$ equiv) was added to the homogenous orange solution, followed by sulfonyl azide $46(0.17 \mathrm{~g}, 0.75 \mathrm{mmol}, 1.5$ equiv) and tert-butyl hydroperoxide $(5.5 \mathrm{M}$ in decane, $25 \mu \mathrm{L}, 0.14 \mathrm{mmol}$, 0.28 equiv). After $5 \mathrm{~min}$, TMDSO (0.13 mL, $0.75 \mathrm{mmol}, 1.5$ equiv) was added dropwise. The resulting dark brown-green to dark green solution was stirred at $23{ }^{\circ} \mathrm{C}$ and the reaction monitored by TLC (AcOEt/hexane 1:40). After completion the reaction was quenched with $\mathrm{H}_{2} \mathrm{O}(2 \mathrm{~mL})$. Sat. $\mathrm{NaHCO}_{3}(2 \mathrm{~mL})$ and brine $(5 \mathrm{~mL})$ were added and the reaction mixture was extracted with AcOEt $(3 \times 10 \mathrm{~mL})$. The combined organic layers were dried over $\mathrm{Na}_{2} \mathrm{SO}_{4}$, filtered and the solvents were removed under reduced pressure ( $p=50 \mathrm{mbar}, T=40^{\circ} \mathrm{C}$ ). The isolated product was purified by column chromatography to afford the desired alkyl azide.

General Procedure for the Co-Catalyzed Hydroazidation of Olefins with TMDSO and sulfonylazide 47 (GP8)

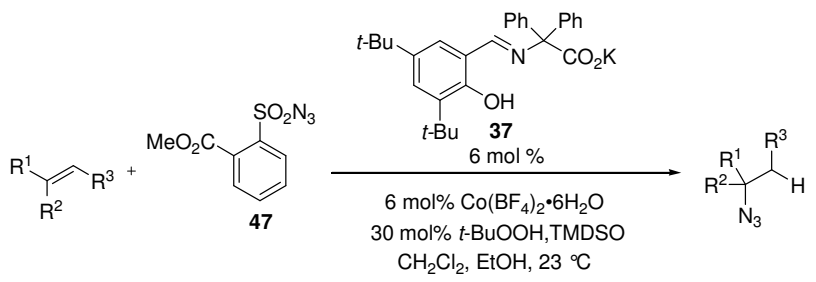

$\mathrm{Co}\left(\mathrm{BF}_{4}\right)_{2} \bullet 6 \mathrm{H}_{2} \mathrm{O}$ (10 mg, $0.030 \mathrm{mmol}, 0.060$ equiv) and ligand 37 (14 mg, $0.030 \mathrm{mmol}, 0.060$ equiv) were dissolved in ethanol $(2.5 \mathrm{~mL})$ at $23{ }^{\circ} \mathrm{C}$ under argon. After $10 \mathrm{~min}$, the alkene $(0.50 \mathrm{mmol}, 1.0$ equiv) was added to the homogenous orange solution, followed by sulfonyl azide $46\left(0.18 \mathrm{~g}, 0.75 \mathrm{mmol}, 1.5\right.$ equiv), $\mathrm{CH}_{2} \mathrm{Cl}_{2}(1.0 \mathrm{~mL})$ and tert-butyl hydroperoxide $(5.5 \mathrm{M}$ in decane, 25 $\mu \mathrm{L}, 0.14 \mathrm{mmol}, 0.28$ equiv). After $5 \mathrm{~min}$, TMDSO $(0.13 \mathrm{~mL}, 0.75 \mathrm{mmol}, 1.5$ equiv) was added dropwise. The resulting dark brown-green to dark green solution was stirred at $23{ }^{\circ} \mathrm{C}$ and the reaction monitored by TLC (AcOEt/hexane 1:40). After completion the reaction was quenched with $\mathrm{H}_{2} \mathrm{O}(2 \mathrm{~mL})$. Sat. $\mathrm{NaHCO}_{3}(2 \mathrm{~mL})$ and brine $(5 \mathrm{~mL})$ were added and the reaction mixture was extracted with AcOEt $(3 \times 10 \mathrm{~mL})$. The combined organic layers were dried over $\mathrm{Na}_{2} \mathrm{SO}_{4}$, filtered and the solvents were removed under reduced pressure $\left(p=50 \mathrm{mbar}, T=40^{\circ} \mathrm{C}\right)$. The isolated product was purified by column chromatography to afford the desired alkyl azide. 


\subsubsection{Hydroazidation Products}

(3-Azido-butyl)-benzene (27) ${ }^{51}$

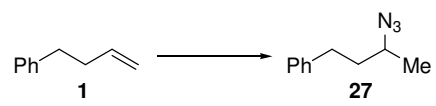

First Lead Result Phenylsilane (65 $\mu \mathrm{L}, 0.52 \mathrm{mmol}, 1.0$ equiv) and ethanesulfonyl azide (200) ( $0.10 \mathrm{~g}, 0.75 \mathrm{mmol}, 1.5 \mathrm{equiv})$ were added to a solution of Co catalyst $20(10 \mathrm{mg}, 0.025 \mathrm{mmol}, 0.050$ equiv) and 4-phenylbutene (1) $(75 \mu \mathrm{L}, 0.50 \mathrm{mmol}, 1.0$ equiv) at $23{ }^{\circ} \mathrm{C}$ under argon. The resulting dark red solution was stirred at $23{ }^{\circ} \mathrm{C}$ and the reaction monitored by TLC (AcOEt/hexane 1:40). After $48 \mathrm{~h}$, the reaction was still not completed and was quenched with $\mathrm{H}_{2} \mathrm{O}(2 \mathrm{~mL}) . \mathrm{Sat} . \mathrm{NaHCO}_{3}(2 \mathrm{~mL})$ and brine $(5 \mathrm{~mL})$ were added and the reaction mixture was extracted with AcOEt $(3 \times 10 \mathrm{~mL})$. The combined organic layers were dried over $\mathrm{Na}_{2} \mathrm{SO}_{4}$, filtered and the solvents were removed under reduced pressure $\left(p=50 \mathrm{mbar}, T=40{ }^{\circ} \mathrm{C}\right)$. The isolated product was purified by column chromatography (AcOEt/hexane 1:60) to afford the desired alkyl azide $\mathbf{3 3}$ (45 $\mathrm{mg}, 0.25 \mathrm{mmol}, 50 \%)$ as a colorless liquid and recovered alkene 1 (11 mg, $0.083 \mathrm{mmol}, 16 \%)$. Following GP5 Azide 33 (79 mg, 0.45 mmol, $90 \%)$ was obtained as a colorless liquid with 4-phenylbutene (1) $(75 \mu \mathrm{L}, 0.50 \mathrm{mmol}, 1.0$ equiv) in $2 \mathrm{~h}$ after purification by column chromatography (solvent gradient: pentane, then pentane/ $\mathrm{Et}_{2} \mathrm{O}$ 60:1). Gas chromatography showed full conversion (>99\%) after $2 \mathrm{~h}$ and a selectivity of 89:11 for the azidation of the alkene compared to simple reduction. Following GP6 Azide 33 (75 mg, 0.43 mmol, 86\%) was obtained as colorless liquid with 4-phenylbutene (1) (75 $\mu \mathrm{L}, 0.50 \mathrm{mmol}, 1.0$ equiv) and TMDSO (0.18 mL, 1.0 mmol, 2.0 equiv) in $3 \mathrm{~h}$ after purification by column chromatography (solvent gradient: pentane, then pentane/Et $\mathrm{O}_{2} \mathrm{O} 0: 1$ ). $\mathrm{Gas}$ chromatography showed full conversion ( $>99 \%$ ) after $3 \mathrm{~h}$ and a selectivity of 96:4 for the azidation of the alkene compared to simple reduction. Following GP7 (slightly modified) Azide 33 ( $83 \mathrm{mg}, 0.47 \mathrm{mmol}, 94 \%)$ was obtained as colorless liquid with 4 phenylbutene (1) $(75 \mu \mathrm{L}, 0.50 \mathrm{mmol}, 1.0$ equiv), TMDSO (88 $\mu \mathrm{L}, 0.50 \mathrm{mmol}, 1.0$ equiv) and azide $52(0.14 \mathrm{~g}, 0.60 \mathrm{mmol}, 1.2$ equiv) in $5 \mathrm{~h}$ after purification by column chromatography (solvent gradient: pentane, then pentane/Et $\mathrm{O}_{2} \mathrm{O}$ 60:1). Following GP8 Azide 33 (80 mg, $0.46 \mathrm{mmol}, 91 \%)$ was obtained as colorless liquid with 4-phenylbutene (1) $(75 \mu \mathrm{L}, 0.50 \mathrm{mmol}, 1.0 \mathrm{equiv})$ in $4 \mathrm{~h}$ after purification by column chromatography (solvent gradient: pentane, then pentane/Et ${ }_{2} \mathrm{O} 60: 1$ ). Following GP5, but with CoSalen Complex 34b Azide 33 (60 mg, $0.34 \mathrm{mmol}, 68 \%)$ was obtained as colorless liquid with 4-phenylbutene (1) (75 $\mu \mathrm{L}, 0.50$ mmol, 1.0 equiv), Co-salen catalyst $\mathbf{3 4 b}(15 \mathrm{mg}, 0.025 \mathrm{mmol}, 0.050$ equiv) in $5 \mathrm{~h}$ after purification by column chromatography (solvent gradient: pentane, then pentane/ $\mathrm{Et}_{2} \mathrm{O}$ 60:1). Separation of the two enantiomers of 33 via HPLC was not directly possible. However, separation of the two enantiomers of the triazole obtained from the cycloaddition reaction of $\mathbf{3 3}$ with phenyl acetylene following a reported procedure ${ }^{52}$ was easy, but the obtained triazole was racemic (Daicel Chiracel OD-H column $250 \times 4.6 \mathrm{~mm}$, hexane/i-PrOH 80:20, flow: $1.0 \mathrm{~mL} / \mathrm{min}, \mathrm{t}_{\mathrm{R}}: 12.0,15.4 \mathrm{~min}$, detector: $254 \mathrm{~nm}$ ).

\section{Gas chromatographic measurements:}

An aliquot of $0.10 \mathrm{~mL}$ of the reaction mixture was diluted with $0.90 \mathrm{~mL}$ hexane and filtered through a short silica gel plug (2.5 $\mathrm{cm}$ ) to remove the catalyst. $2 \mu \mathrm{L}$ of the obtained solution were injected in the gas chromatographer (Varian 3300 gas chromatographer) and the following temperature program was followed:- $10 \mathrm{~min}$ at $60{ }^{\circ} \mathrm{C}$ - to $110{ }^{\circ} \mathrm{C}$ with a temperature gradient of $8{ }^{\circ} \mathrm{C} / \mathrm{min}-1 \mathrm{~min}$ at $110{ }^{\circ} \mathrm{C}$ - to $180{ }^{\circ} \mathrm{C}$ with a temperature gradient of $20{ }^{\circ} \mathrm{C} / \mathrm{min}$. Using this procedure, 4-phenylbutene (1) eluted after $12.8 \mathrm{~min}$, 4-phenylbutane (44) after $13.5 \mathrm{~min}$ and (3-azido-butyl)-benzene (33) after $23.8 \mathrm{~min}$.

$\mathrm{R}_{\mathrm{f}}$ (AcOEt/hexane 1:40) 0.30; ${ }^{1} \mathrm{H}$ NMR $\left(\mathrm{CDCl}_{3}, 300 \mathrm{MHz}\right) \delta$ 7.37-7.32 (m, 2H, Ar H), 7.27-7.22 (m, 3H, Ar H), 3.52-3.43 (m, $\left.1 \mathrm{H}, \mathrm{CHN}_{3}\right), 2.86-2.66\left(\mathrm{~m}, 2 \mathrm{H}, \mathrm{PhCH}_{2}\right), 1.92-1.75\left(\mathrm{~m}, 2 \mathrm{H}, \mathrm{CH}_{2} \mathrm{CHN}_{3}\right), 1.33\left(\mathrm{~d}, J=6.6 \mathrm{~Hz}, 3 \mathrm{H}, \mathrm{CH}_{3}\right) ;{ }^{13} \mathrm{C} \mathrm{NMR}\left(\mathrm{CDCl}_{3}, 75 \mathrm{MHz}\right)$ $\delta$ 141.1, 128.4, 128.3, 126.0, 57.1, 37.8, 32.2, 19.4; IR v $3064(\mathrm{w}), 3028(\mathrm{~m}), 2972(\mathrm{~m}), 2931(\mathrm{~m}), 2862(\mathrm{w}), 2100(\mathrm{~s}), 1947(\mathrm{w})$, $1881(\mathrm{w}), 1805(\mathrm{w}), 1604(\mathrm{w}), 1496(\mathrm{~m}), 1454(\mathrm{~m}), 1380(\mathrm{~m}), 1330(\mathrm{~m}), 1250(\mathrm{~s}), 1125(\mathrm{w}), 1047(\mathrm{w}), 1031(\mathrm{w}), 916(\mathrm{w}), 857$ (w), $832(\mathrm{w}), 800(\mathrm{w}), 747(\mathrm{~m}), 699(\mathrm{~s}), 578(\mathrm{w}), 562(\mathrm{w}), 515(\mathrm{w}), 466(\mathrm{w})$.

(3-Azido-3-methyl-butyl)-naphthalene (229)

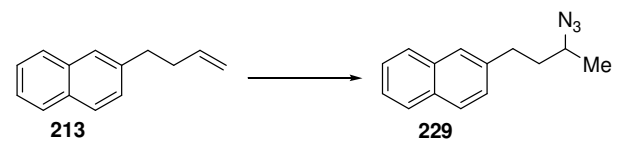

Following GP5 Azide 229 (80 mg, $0.36 \mathrm{mmol}, 72 \%$ ) was obtained as an oil with (3-methyl-but-3-enyl)-naphthalene (213) (89 $\mathrm{mg}, 0.49 \mathrm{mmol}, 1.0$ equiv) in $8 \mathrm{~h}$ after purification by column chromatography (solvent gradient: pentane, then pentane/Et $\mathrm{O}_{2}$ 60:1). Following GP6 Azide 229 (76 mg, $0.34 \mathrm{mmol}, 69 \%)$ and (3-methyl-but-3-enyl)-naphthalene (213) $(23 \mathrm{mg}, 0.13 \mathrm{mmol}$, $26 \%$ recovery) were obtained as oils with (3-methyl-but-3-enyl)-naphthalene (213) (77 mg, $0.53 \mathrm{mmol}, 1.0$ equiv) and TMDSO $(0.18 \mathrm{~mL}, 1.0 \mathrm{mmol}, 2.0$ equiv) in $12 \mathrm{~h}$ after purification by column chromatography (solvent gradient: pentane, then pentane/Et $\left.{ }_{2} \mathrm{O} 60: 1\right) . \mathrm{R}_{\mathrm{f}}\left(\mathrm{AcOEt} / \mathrm{h}\right.$ exane 1:40) 0.30; ${ }^{1} \mathrm{H} \mathrm{NMR}\left(\mathrm{CDCl}_{3}, 300 \mathrm{MHz}\right) \delta 7.85-7.79(\mathrm{~m}, 3 \mathrm{H}$, naphthyl H), $7.65(\mathrm{~s}, 1 \mathrm{H}$, naphthyl $\mathrm{H}), 7.51-7.42(\mathrm{~m}, 3 \mathrm{H}$, naphthyl $\mathrm{H}), 7.35(\mathrm{dd}, J=8.4,1.9 \mathrm{~Hz}, 1 \mathrm{H}$, naphthyl H), 3.52-3.45 (m, $1 \mathrm{H}, \mathrm{CHN}), 2.99-2.80(\mathrm{~m}$, $2 \mathrm{H}$, naphthyl- $\left.\mathrm{CH}_{2}\right), 2.00-1.82\left(\mathrm{~m}, 2 \mathrm{H}, \mathrm{CH}_{2} \mathrm{CHN}_{3}\right), 1.33\left(\mathrm{~d}, J=6.5 \mathrm{~Hz}, 3 \mathrm{H}, \mathrm{CH}_{3}\right) ;{ }^{13} \mathrm{C} \mathrm{NMR}\left(\mathrm{CDCl}_{3}, 75 \mathrm{MHz}\right) \delta 139.0,133.9$, 132.3, 128.4 127.9, 127.7, 127.4, 126.8, 126.3, 125.6, 57.4, 38.0, 32.7, 19.8; IR v 3052 (w), 3019 (w), 2969 (m), $2929(\mathrm{~m}), 2861$ (w), $2099(\mathrm{~s}), 1633(\mathrm{w}), 1600(\mathrm{w}), 1507(\mathrm{~m}), 1452(\mathrm{~m}), 1379(\mathrm{~m}), 1332(\mathrm{~m}), 1270(\mathrm{~m}), 1246(\mathrm{~m}), 1125(\mathrm{w}), 1059(\mathrm{w}), 1018(\mathrm{w})$, 960 (w), 891 (w), 854 (m), 818 (s), 746 (s), 650 (w), 621 (w), 560 (w), 477 (m); MS (EI-HIRES) 182.0974 (M-HN 3 ), 196.1130 $\left(\mathrm{M}-\mathrm{HN}_{2}\right), 197.1201\left(\mathrm{M}-\mathrm{N}_{2}\right), 219.1375\left(\mathrm{M}-\mathrm{HN}_{2}+\mathrm{Na}\right)$; Anal. calcd for $\mathrm{C}_{14} \mathrm{H}_{15} \mathrm{~N}_{3}: \mathrm{C}, 74.64 ; \mathrm{H}, 6.71 ; \mathrm{N}, 18.65$. Found: C, 74.71; H, $6.88 ; \mathrm{N}, 18.37$. 


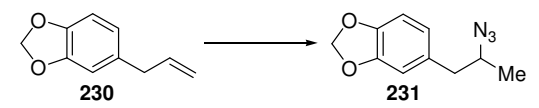

Following GP5 Azide 231 (67 mg, $0.33 \mathrm{mmol}, 66 \%$ ) and safrole (230) (9 mg, $0.06 \mathrm{mmol}, 11 \%$ recovery) were obtained as oils with safrole (230) $\left(74 \mu \mathrm{L}, 0.50 \mathrm{mmol}, 1.0\right.$ equiv) in $20 \mathrm{~h}$ after purification by column chromatography (pentane/Et $\left.{ }_{2} \mathrm{O} 120: 1\right)$. Following GP6 Azide 231 (64 mg, $0.31 \mathrm{mmol}, 62 \%)$ and safrole (230) (12 mg, $0.075 \mathrm{mmol}, 15 \%$ recovery) were obtained as oils with safrole (230) $(74 \mu \mathrm{L}, 0.50 \mathrm{mmol}, 1.0$ equiv) and TMDSO $(0.18 \mathrm{~mL}, 1.0 \mathrm{mmol}, 2.0$ equiv) in $20 \mathrm{~h}$ after purification by column chromatography (pentane/Et $\left.{ }_{2} \mathrm{O} 120: 1\right) . \mathrm{R}_{\mathrm{f}}$ (AcOEt/hexane 1:40) $0.25 ;{ }^{1} \mathrm{H}$ NMR $\left(\mathrm{CDCl}_{3}, 300 \mathrm{MHz}\right) \delta 6.96-6.63(\mathrm{~m}, 3 \mathrm{H}, \mathrm{Ar}$ $\mathrm{H}), 5.94\left(\mathrm{~s}, 2 \mathrm{H}\right.$, acetal-H), 3.66-3.60 (m, $\left.1 \mathrm{H}, \mathrm{CHN}_{3}\right), 2.77-2.61\left(\mathrm{~m}, 2 \mathrm{H}, \mathrm{ArCH}_{2}\right), 1.25\left(\mathrm{~d}, \mathrm{~J}=6.6 \mathrm{~Hz}, 3 \mathrm{H}, \mathrm{CH}_{3}\right) ;{ }^{13} \mathrm{C} \mathrm{NMR}\left(\mathrm{CDCl}_{3}\right.$, $75 \mathrm{MHz}) \delta 147.5,146.2,131.3,122.2,109.5,108.2,100.9,59.2,42.3,19.1$; IR $v 2973$ (w), 2894 (w), 2105 (s), 1844 (w), 1608 (w), 1503 (s), 1490 (s), 1443 (m), 1379 (w), 1362 (w), 1327 (w), 1250 (s), 1190 (m), 1123 (w), 1100 (w), 1040 (s), 931 (m), 913

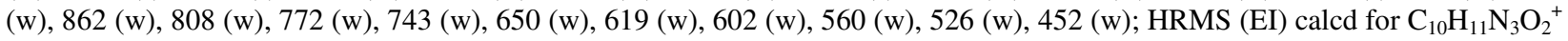
(M): 205.0846, found 205.0846; Anal. calcd for $\mathrm{C}_{10} \mathrm{H}_{11} \mathrm{~N}_{3} \mathrm{O}_{2}$ : C, 58.53; H, 5.40; N, 20.48. Found: C, 58.53; H, 5.52; N, 20.41.

(3-Azido-butoxy)-tert-butyl-diphenyl-silane (232)

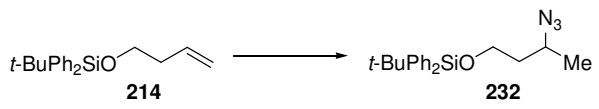

Following GP5 Azide 232 (127 mg, $0.359 \mathrm{mmol}, 73 \%$ ) and butoxy-tert-butyl-diphenyl-silane (25 mg, 0.080 mmol, 16\%) were obtained as oils with but-3-enyloxy-tert-butyl-diphenyl-silane (214) (152 $\mathrm{mg}, 0.490 \mathrm{mmol}, 1.00 \mathrm{equiv})$ in $3 \mathrm{~h}$ after purification by column chromatography (solvent gradient: pentane, then pentane/ $\mathrm{Et}_{2} \mathrm{O}$ 60:1). Following GP6 Azide 232 (146 mg, $0.413 \mathrm{mmol}$, $85 \%)$ and butoxy-tert-butyl-diphenyl-silane $(9 \mathrm{mg}, 0.03 \mathrm{mmol}, 6 \%)$ were obtained as oils with but-3-enyloxy-tert-butyl-diphenylsilane (214) (150 mg, $0.483 \mathrm{mmol}, 1.00$ equiv) and TMDSO (0.26 mL, $1.5 \mathrm{mmol}, 3.0$ equiv) in $3 \mathrm{~h}$ after purification by column chromatography (solvent gradient: pentane, then pentane/Et $\left.{ }_{2} \mathrm{O} 60: 1\right) . \mathrm{R}_{\mathrm{f}}\left(\mathrm{AcOEt} /\right.$ hexane 1:40) 0.27; ${ }^{1} \mathrm{H} \mathrm{NMR}\left(\mathrm{CDCl}{ }_{3}, 300 \mathrm{MHz}\right)$ א 7.70-7.66 (m, $4 \mathrm{H}, \mathrm{Ar} \mathrm{H}), 7.45-7.38(\mathrm{~m}, 6 \mathrm{H}, \mathrm{Ar} \mathrm{H}), 3.81-3.70\left(\mathrm{~m}, 3 \mathrm{H}, \mathrm{CH}_{2} \mathrm{O}\right.$ and $\left.\mathrm{CHN}_{3}\right), 1.74-1.67\left(\mathrm{~m}, 2 \mathrm{H}, \mathrm{CH}_{2} \mathrm{CHN}_{3}\right), 1.29(\mathrm{~d}$,

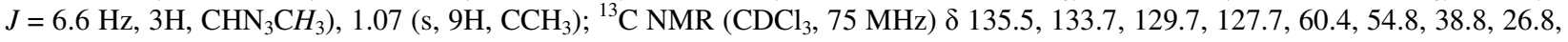
19.6, 19.2; IR v $3070(\mathrm{w}), 3049(\mathrm{w}), 2959(\mathrm{~m}), 2931(\mathrm{~m}), 2878(\mathrm{~m}), 2857(\mathrm{~m}), 2103(\mathrm{~s}), 1959(\mathrm{w}), 1887(\mathrm{w}), 1822(\mathrm{w}), 1657(\mathrm{w})$, $1589(\mathrm{w}), 1472(\mathrm{~m}), 1462(\mathrm{w}), 1428(\mathrm{~m}), 1389(\mathrm{w}), 1361(\mathrm{w}), 1332(\mathrm{w}), 1268(\mathrm{w}), 1188(\mathrm{w}), 1112(\mathrm{~s}), 1007(\mathrm{w}), 997(\mathrm{w}), 982$ (w), 939 (w), 899 (w), 868 (w), 823 (m), 737 (m), 702 (s), 614 (m), 505 (s), 488 (m); MS (ESI) 354.3 (M+H), 376.3 (M+Na); Anal. calcd for $\mathrm{C}_{20} \mathrm{H}_{27} \mathrm{~N}_{3} \mathrm{OSi}$ : C, 67.95; H, 7.70; N, 11.89. Found: C, 68.16; H, 7.66; N, 11.91 .

\section{(3-Azido-butoxy)-tert-butyl-diphenyl-silane (233)}

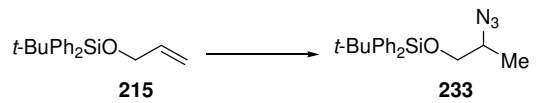

Following GP5 Azide 233 (93 mg, 0.27 mmol, 55\%, about 95\% purity in ${ }^{1} H$-NMR) and propoxy-tert-butyl-diphenyl-silane (36 $\mathrm{mg}, 0.12 \mathrm{mmol}, 24 \%$ ) were obtained as oils with prop-3-enyloxy-tert-butyl-diphenyl-silane (215) (148 $\mathrm{mg}, 0.499 \mathrm{mmol}, 1.00$

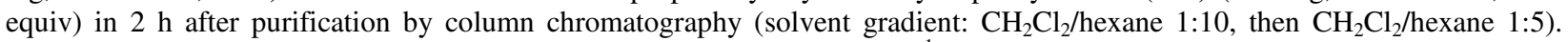
Following GP6 Azide 233 (114 mg, $0.34 \mathrm{mmol}, 67 \%$, about 90\% purity in ${ }^{1} \mathrm{H}$-NMR), propoxy-tert-butyl-diphenyl-silane (7 mg, $0.02 \mathrm{mmol}, 4 \%)$ and prop-3-enyloxy-tert-butyl-diphenyl-silane (215) (18 $\mathrm{mg}, 0.061 \mathrm{mmol}, 12 \%$ recovery) were obtained as oils with prop-3-enyloxy-tert-butyl-diphenyl-silane (215) (147 mg, $0.496 \mathrm{mmol}, 1.00$ equiv) and TMDSO (0.18 mL, $1.0 \mathrm{mmol}, 2.0$ equiv) in $18 \mathrm{~h}$ after purification by column chromatography (solvent gradient: $\mathrm{CH}_{2} \mathrm{Cl}_{2} /$ hexane 1:10, then $\mathrm{CH}_{2} \mathrm{Cl}_{2} / \mathrm{hexane}^{1: 5}$ ). Following GP7 Azide 233 (32 mg, $0.95 \mathrm{mmol}$, 19\%, about 95\% purity in ${ }^{1} \mathrm{H}$ NMR), was obtained as a colorless oil with prop-3enyloxy-tert-butyl-diphenyl-silane (215) (148 mg, $0.499 \mathrm{mmol}, 1.00$ equiv) in $18 \mathrm{~h}$ after purification by column chromatography (solvent gradient: $\mathrm{CH}_{2} \mathrm{Cl}_{2}$ /hexane 1:10, then $\mathrm{CH}_{2} \mathrm{Cl}_{2}$ /hexane 1:5). Following GP8 Azide 233 (89 mg, 0.22 mmol, 44\%, about 90\% purity in ${ }^{1} \mathrm{H}$-NMR), propoxy-tert-butyl-diphenyl-silane (18 $\left.\mathrm{mg}, 0.060 \mathrm{mmol}, 12 \%\right)$ and prop-3-enyloxy-tert-butyl-diphenyl-silane (215) (5 mg, $0.02 \mathrm{mmol}, 4 \%$ recovery) were obtained as oils with prop-3-enyloxy-tert-butyl-diphenyl-silane (215) (148 mg, 0.499 mmol, 1.00 equiv) in $18 \mathrm{~h}$ after purification by column chromatography (solvent gradient: $\mathrm{CH}_{2} \mathrm{Cl}_{2} / \mathrm{hexane} 1: 10$, then $\mathrm{CH}_{2} \mathrm{Cl}_{2}$ /hexane 1:5). Analytically pure samples of the desired product $\mathbf{2 3 3}$ were obtained after a second purification by column chromatography (solvent gradient: $\mathrm{CH}_{2} \mathrm{Cl}_{2}$ /hexane 1:10, then $\mathrm{CH}_{2} \mathrm{Cl}_{2}$ /hexane 1:5). $\mathrm{R}_{\mathrm{f}}(\mathrm{AcOEt} /$ hexane $1: 40)$ 0.30; ${ }^{1} \mathrm{H} \mathrm{NMR}$ $\left(\mathrm{CDCl}_{3}, 300 \mathrm{MHz}\right) \delta$ 7.71-7.66 (m, 4H, Ar H), 7.47-7.37 (m, 6H, Ar H), 3.67-3.53 (m, 3H, $\mathrm{CH}_{2} \mathrm{O}$ and $\left.\mathrm{CHN}_{3}\right), 1.17(\mathrm{~d}, J=6.5 \mathrm{~Hz}$, $\left.3 \mathrm{H}, \mathrm{CHN}_{3} \mathrm{CH}_{3}\right), 1.08\left(\mathrm{~s}, 9 \mathrm{H}, \mathrm{CCH}_{3}\right) ;{ }^{13} \mathrm{C} \mathrm{NMR}\left(\mathrm{CDCl}_{3}, 75 \mathrm{MHz}\right) \delta 135.5,133.0,129.7,127.6,68.0,58.9,26.8,19.3,16.0 ; \mathrm{IR} v$ $3070(\mathrm{w}), 3050(\mathrm{w}), 2959(\mathrm{~m}), 2931$ (m), $2893(\mathrm{w}), 2858(\mathrm{~m}), 2123$ (s), 2109 (s), $1890(\mathrm{w}), 1824(\mathrm{w}), 1652(\mathrm{w}), 1589(\mathrm{w}), 1472$ (w), 1463 (w), $1428(\mathrm{~m}), 1390(\mathrm{w}), 1362(\mathrm{w}), 1332(\mathrm{w}), 1271(\mathrm{~m}), 1188(\mathrm{w}), 1112(\mathrm{~s}), 1022(\mathrm{w}), 1008(\mathrm{w}), 938(\mathrm{w}), 912(\mathrm{~m}), 824$ (m), 805 (w), 741 (s), 702 (s), 615 (m), 504 (s), 489 (m); MS (ESI) $339.2(\mathrm{M}), 362.0(\mathrm{M}+\mathrm{Na})$; Anal. calcd for $\mathrm{C}_{19} \mathrm{H}_{25} \mathrm{~N}_{3} \mathrm{OSi}: \mathrm{C}_{\text {, }}$ 67.22; H, 7.42; N, 12.38. Found: C, 67.48; H, 7.56; N, 12.29.

(2-Azido-propoxymethyl)-benzene (234)

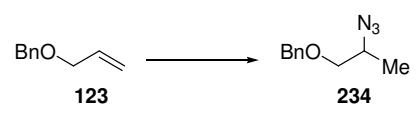


Following GP5 Azide 234 (33 mg, $0.17 \mathrm{mmol}, 35 \%$ ) and allyl benzyl ether (123) (14 mg, $0.094 \mathrm{mmol}, 19 \%$ recovery) were obtained as oils with allyl benzyl ether $(\mathbf{1 2 3})(77 \mu \mathrm{L}, 0.50 \mathrm{mmol}, 1.0$ equiv) in $48 \mathrm{~h}$ after purification by column chromatography (pentane/ $\mathrm{Et}_{2} \mathrm{O}$ 100:1). Following GP6 Azide 234 (37 mg, $\left.0.19 \mathrm{mmol}, 39 \%\right)$ and allyl benzyl ether (123) (16 mg, $0.11 \mathrm{mmol}, 22 \%$ recovery) were obtained as oils with allyl benzyl ether $(\mathbf{1 2 3})(77 \mu \mathrm{L}, 0.50 \mathrm{mmol}, 1.0$ equiv) and TMDSO $(0.26 \mathrm{~mL}, 1.5 \mathrm{~mol}, 3.0$ equiv) in $48 \mathrm{~h}$ after purification by column chromatography (pentane/Et ${ }_{2} \mathrm{O}$ 100:1). Following GP7 Azide 234 was detected only as a minor product $(<20 \%)$ in the crude ${ }^{1} H$-NMR of the reaction mixture and was not isolated. Following GP8 Azide 234 (27 $\mathrm{mg}, 0.14 \mathrm{mmol}, 28 \%)$ and allyl benzyl ether (123) $(8 \mathrm{mg}, 0.05 \mathrm{mmol}, 10 \%$ recovery) were obtained as oils with allyl benzyl ether (123) $\left(77 \mu \mathrm{L}, 0.50 \mathrm{mmol}, 1.0\right.$ equiv) in $48 \mathrm{~h}$ after purification by column chromatography (pentane/Et $\left.\mathrm{E}_{2} \mathrm{O} 100: 1\right)$. $\mathrm{R}_{\mathrm{f}}$ (AcOEt/hexane 1:40) 0.25; ${ }^{1} \mathrm{H}$ NMR $\left(\mathrm{CDCl}_{3}, 300 \mathrm{MHz}\right) \delta 7.38-7.28(\mathrm{~m}, 5 \mathrm{H}, \mathrm{Ar} \mathrm{H}), 4.58\left(\mathrm{~s}, 2 \mathrm{H}, \mathrm{PhCH}_{2}\right), 3.74-3.68(\mathrm{~m}, 1 \mathrm{H}$, $\left.\mathrm{CHN}_{3}\right), 3.54-3.42\left(\mathrm{~m}, 2 \mathrm{H}, \mathrm{CH}_{2} \mathrm{O}\right), 1.22\left(\mathrm{~d}, J=6.8 \mathrm{~Hz}, 3 \mathrm{H}, \mathrm{CH}_{3}\right) ;{ }^{13} \mathrm{C} \mathrm{NMR}\left(\mathrm{CDCl}_{3}, 75 \mathrm{MHz}\right) \delta 137.7,128.3,127.6,127.5,73.8$, 73.3, 57.0, 16.3; IR $v 3064(\mathrm{w}), 3030(\mathrm{w}), 2976(\mathrm{w}), 2861(\mathrm{w}), 2124(\mathrm{~s}), 2103(\mathrm{~s}), 1496(\mathrm{w}), 1453(\mathrm{w}), 1379(\mathrm{w}), 1362(\mathrm{w}), 1330$ (w), 1266 (m), 1206 (w), 1102 (m), 1028 (w), 998 (w), 913 (s), 743 (s), 698 (m), 651 (w), $621(\mathrm{w}), 558$ (w), 458 (w), 441 (w); MS (EI-HIRES) $162.0912\left(\mathrm{M}-\mathrm{HN}_{2}\right), 163.0947\left(\mathrm{M}-\mathrm{N}_{2}\right)$; Anal. calcd for $\mathrm{C}_{10} \mathrm{H}_{13} \mathrm{~N}_{3} \mathrm{O}: \mathrm{C}, 62.81 ; \mathrm{H}, 6.85 ; \mathrm{N}, 21.97$. Found: C, 63.04; H, $7.00 ; \mathrm{N}, 21.79$.

4-Azido-pentanoic acid benzyl ester (235)

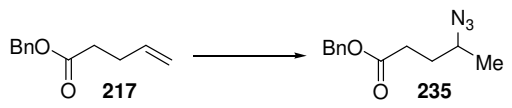

Following GP5 Azide 235 (86 mg, 0.37 mmol, 75\%) was obtained as an oil with pent-4-enoic acid benzyl ester (217) (94 mg, $0.49 \mathrm{mmol}, 1.0$ equiv) in $10 \mathrm{~h}$ after purification by column chromatography (solvent gradient: $\mathrm{CH}_{2} \mathrm{Cl}_{2} / \mathrm{hexane} 1: 2$, then $\mathrm{CH}_{2} \mathrm{Cl}_{2} /$ hexane 1:1). Following GP6 Azide 235 (90 mg, $\left.0.39 \mathrm{mmol}, 77 \%\right)$ was obtained as an oil with pent-4-enoic acid benzyl ester (217) (95 mg, $0.50 \mathrm{mmol}, 1.0$ equiv) and TMDSO (0.18 mL, $1.0 \mathrm{mmol}, 2.0$ equiv) in $10 \mathrm{~h}$ after purification by column chromatography (solvent gradient: $\mathrm{CH}_{2} \mathrm{Cl}_{2} /$ hexane 1:2, then $\mathrm{CH}_{2} \mathrm{Cl}_{2} /$ hexane 1:1). $\mathrm{R}_{\mathrm{f}}(\mathrm{AcOEt} /$ hexane $1: 10) 0.40 ;{ }^{1} \mathrm{H} \mathrm{NMR}\left(\mathrm{CDCl}_{3}\right.$, $300 \mathrm{MHz}) \delta$ 7.42-7.31 (m, 5H, Ar H), $5.14\left(\mathrm{~s}, 2 \mathrm{H}, \mathrm{PhCH}_{2}\right), 3.55-3.48\left(\mathrm{~m}, 1 \mathrm{H}, \mathrm{CHN}_{3}\right), 2.51-2.42\left(\mathrm{~m}, 2 \mathrm{H}, \mathrm{CH}_{2} \mathrm{CO}\right), 1.92-1.71(\mathrm{~m}$, $\left.2 \mathrm{H}, \mathrm{CH}_{2} \mathrm{CHN}_{3}\right), 1.29\left(\mathrm{~d}, J=6.5 \mathrm{~Hz}, 3 \mathrm{H}, \mathrm{CH}_{3}\right) ;{ }^{13} \mathrm{C} \mathrm{NMR}\left(\mathrm{CDCl}_{3}, 75 \mathrm{MHz}\right) \delta 172.5,135.6,128.4,128.1,128.1,66.3,57.0,31.2$, 30.8, 19.3; IR v $3336(\mathrm{w}), 3066(\mathrm{w}), 3034(\mathrm{w}), 2972(\mathrm{~m}), 2102(\mathrm{~s}), 1736(\mathrm{~s}), 1498(\mathrm{w}), 1455(\mathrm{~m}), 1382(\mathrm{~m}), 1339(\mathrm{~m}), 1269(\mathrm{~s})$, 1167 (s), 1127 (m), 1028 (m), 965 (m), 913 (w), 868 (w), 739 (m), 697 (m); MS (EI-HIRES) 204.1027 (M-HN $)_{2}, 205.1074(\mathrm{M}-$ $\mathrm{N}_{2}$ ); Anal. calcd for $\mathrm{C}_{12} \mathrm{H}_{15} \mathrm{~N}_{3} \mathrm{O}_{2}: \mathrm{C}, 61.79 ; \mathrm{H}, 6.48 ; \mathrm{N}, 18.01$. Found: $\mathrm{C}, 62.04 ; \mathrm{H}, 6.40 ; \mathrm{N}, 17.88$.

4-Azido-1-phenyl-pentan-1-one (236)

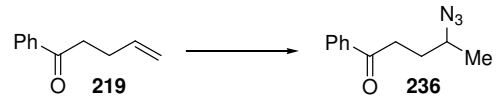

Following GP5 Azide 236 (51 mg, 0.25 mmol, 49\%) and 1-phenyl-pent-4-en-1-one (219) (26 mg, 0.16 mmol, $32 \%$ recovery) were obtained as oils with 1-phenyl-pent-4-en-1-one (219) $(82 \mathrm{mg}, 0.51 \mathrm{mmol}, 1.0$ equiv) in $24 \mathrm{~h}$ after purification by column chromatography (solvent gradient: $\mathrm{CH}_{2} \mathrm{Cl}_{2}$ /hexane 1:2, then $\mathrm{CH}_{2} \mathrm{Cl}_{2}$ /hexane 1:1). Following GP6 Azide 236 (46 mg, $0.23 \mathrm{mmol}$, 46\%) and 1-phenyl-pent-4-en-1-one (219) $(30 \mathrm{mg}, 0.19 \mathrm{mmol}, 38 \%$ recovery) were obtained as oils with 1-phenyl-pent-4-en-1one (219) (79 mg, $0.49 \mathrm{mmol}, 1.0$ equiv) and TMDSO (0.26 mL, $1.5 \mathrm{~mol}, 3.0$ equiv) in $24 \mathrm{~h}$ after purification by column chromatography (solvent gradient: $\mathrm{CH}_{2} \mathrm{Cl}_{2} /$ hexane 1:2, then $\mathrm{CH}_{2} \mathrm{Cl}_{2} /$ hexane 1:1). $\mathrm{R}_{\mathrm{f}}(\mathrm{AcOEt} /$ hexane $1: 10) 0.40 ;{ }^{1} \mathrm{H} \mathrm{NMR}\left(\mathrm{CDCl}_{3}\right.$,

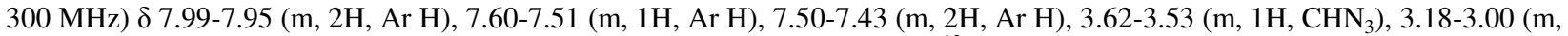

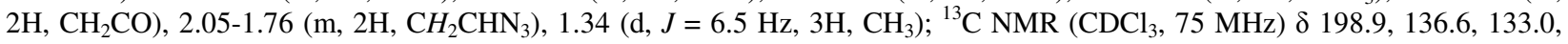
128.5, 127.9, 57.3, 34.8, 30.4, 19.6; IR v $3353(\mathrm{w}), 3062(\mathrm{w}), 2972(\mathrm{w}), 2931(\mathrm{w}), 2105$ (s), $1686(\mathrm{~s}), 1597(\mathrm{w}), 1580(\mathrm{w}), 1448$ (m), $1413(\mathrm{w}), 1381(\mathrm{w}), 1342(\mathrm{w}), 1218(\mathrm{~m}), 1208(\mathrm{~m}), 1180(\mathrm{w}), 1126(\mathrm{w}), 1071(\mathrm{w}), 1017(\mathrm{w}), 1002(\mathrm{w}), 970(\mathrm{w}), 913(\mathrm{~m})$, $744(\mathrm{~s}), 690(\mathrm{~m}), 654(\mathrm{w}), 616(\mathrm{w}), 564(\mathrm{w}), 506(\mathrm{w})$; MS (EI-HIRES) $161.0924\left(\mathrm{M}-\mathrm{N}_{3}\right), 175.0989$ (M-N ${ }_{2}$ ); Anal. calcd for $\mathrm{C}_{11} \mathrm{H}_{13} \mathrm{~N}_{3} \mathrm{O}: \mathrm{C}, 65.01 ; \mathrm{H}, 6.45 ; \mathrm{N}, 20.67$. Found: C, 64.82; H, 6.59; N, 20.76.

(3-Azido-3-methyl-butyl)-benzene $(237)^{51}$

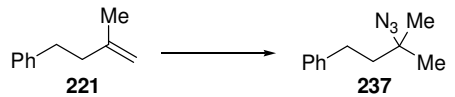

Following GP5 Azide 237 (81 mg, $0.43 \mathrm{mmol}, 86 \%$ ) was obtained as an oil with (3-methyl-but-3-enyl)-benzene (221) (73 mg, $0.50 \mathrm{mmol}, 1.0$ equiv) in $3 \mathrm{~h}$ after purification by column chromatography (solvent gradient: pentane, then pentane/Et $2 \mathrm{O} 60: 1$ ). Following GP6 Azide 237 (90 mg, $0.48 \mathrm{mmol}, 90 \%$ ) was obtained as an oil with (3-methyl-but-3-enyl)-benzene (221) (77 mg, $0.53 \mathrm{mmol}, 1.0$ equiv) and TMDSO $(0.18 \mathrm{~mL}, 1.0 \mathrm{mmol}, 2.0$ equiv) in $12 \mathrm{~h}$ after purification by column chromatography (solvent gradient: pentane, then pentane/Et $2 \mathrm{O} 60: 1) . \mathrm{R}_{\mathrm{f}}$ (AcOEt/hexane 1:40) $0.30 ;{ }^{1} \mathrm{H}$ NMR $\left(\mathrm{CDCl}_{3}, 300 \mathrm{MHz}\right) \delta 7.34-7.20(\mathrm{~m}, 5 \mathrm{H}, \mathrm{Ar}$

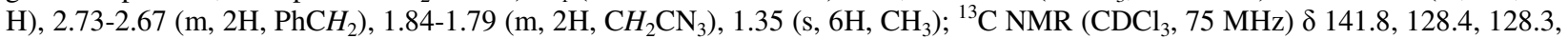
125.9, 61.4, 43.5, 30.7, 26.0; IR v 3329 (w), 3086 (w), 3062 (w), 3027 (w), 2972 (m), 2099 (s), 1603 (w), $1497(w), 1454(\mathrm{~m})$, $1388(\mathrm{w}), 1370(\mathrm{~m}), 1255(\mathrm{~s}), 1204(\mathrm{~m}), 1163(\mathrm{w}), 1124(\mathrm{w}), 1073(\mathrm{w}), 1030(\mathrm{w}), 871(\mathrm{w}), 850(\mathrm{w}), 822(\mathrm{w}), 767(\mathrm{w}), 744(\mathrm{~m})$, $699(\mathrm{~s}), 631(\mathrm{w}), 565(\mathrm{w}), 512(\mathrm{w})$.

2-Azido-2,6,6-trimethyl-bicyclo[3.1.1]heptane (238) 


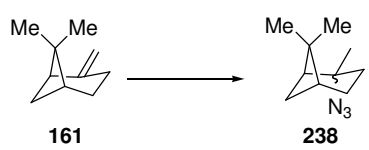

Following GP5 Azide 238 ( $80 \mathrm{mg}, 0.45 \mathrm{mmol}, 89 \%$, mixture of diastereoisomers: 4:1) was obtained as an oil with $\beta$-pinene (161) $\left(78 \mu \mathrm{L}, 0.50 \mathrm{mmol}, 1.0\right.$ equiv) in $12 \mathrm{~h}$ after purification by column chromatography (pentane/Et ${ }_{2} \mathrm{O}$ 100:1). Following GP6 Azide 238 (68 mg, $0.38 \mathrm{mmol}, 76 \%$, mixture of diastereoisomers: 4:1) was obtained as an oil with $\beta$-pinene (161) (78 $\mu \mathrm{L}, 0.50$ mmol, 1.0 equiv) and TMDSO ( $0.26 \mathrm{~mL}, 1.5 \mathrm{~mol}, 3.0$ equiv) in $12 \mathrm{~h}$ catalyst after purification by column chromatography (pentane/ $\mathrm{Et}_{2} \mathrm{O}$ 100:1). Caution: As the desired product displayed a very high tendency to co-evaporate with many solvents, the following work-up was substituted to the one described in the general procedure: After completion (12 h) the reaction was quenched with $\mathrm{H}_{2} \mathrm{O}(2 \mathrm{~mL})$. Sat. $\mathrm{NaHCO}_{3}(2 \mathrm{~mL})$ and brine $(5 \mathrm{~mL})$ were added and the reaction mixture was extracted with $\mathrm{Et}_{2} \mathrm{O}$ $(3 \times 10 \mathrm{~mL})$. The combined organic layers were washed with water $(3 \times 10 \mathrm{~mL})$ and brine $(10 \mathrm{~mL})$, dried over $\mathrm{Na}_{2} \mathrm{SO}_{4}$, filtered and the solvents were removed under reduced pressure $\left(p=700 \mathrm{mbar}, T=40{ }^{\circ} \mathrm{C}\right)$. After column chromatography, the fractions were not fully concentrated and the yield was determined via ${ }^{1} H$-NMR. $\mathrm{R}_{\mathrm{f}}(\mathrm{AcOEt} / \mathrm{hexane} 1: 40) 0.40 ;{ }^{1} \mathrm{H} \mathrm{NMR}\left(\mathrm{CDCl} \mathrm{N}_{3}, 300 \mathrm{MHz}\right) \delta$ 2.27-2.19 (m, $1 \mathrm{H}, \mathrm{CH}_{2}$ or $\left.\mathrm{CH}\right), 1.97-1.64\left(\mathrm{~m}, 7 \mathrm{H}, \mathrm{CH}_{2}\right.$ or $\left.\mathrm{CH}\right), 1.42\left(\mathrm{~s}, 3 \mathrm{H}(80 \%), \mathrm{CH}_{3}\right.$ 1. diastereoisomer), $1.41(\mathrm{~s}, 3 \mathrm{H}(20 \%)$, $\mathrm{CH}_{3}$ 2. diastereoisomer), 1.26 (s, 3H (80\%), $\mathrm{CH}_{3}$ 1. diastereoisomer), 1.23 (s, 3H (20\%), $\mathrm{CH}_{3}$ 2. diastereoisomer), 0.99 (s, $3 \mathrm{H}$, $\left.\mathrm{CH}_{3}\right) ;{ }^{13} \mathrm{C} \mathrm{NMR}\left(\mathrm{CDCl}_{3}, 75 \mathrm{MHz}\right) \delta 67.6,51.5,40.2,38.4,28.9,28.7,27.8,27.3,25.1,24.1,23.4,23.2 ; \mathrm{IR} v 2917(\mathrm{~m}), 2099(\mathrm{~s})$, 1465 (w), 1378 (w), 1252 (m), 1161 (w), 1133 (w), 1095 (w), 1075 (w), 1011 (w), 911 (w), 831 (w); MS (EI-HIRES) 137.1346 $\left(\mathrm{M}-\mathrm{N}_{3}\right)$; Anal. calcd for $\mathrm{C}_{10} \mathrm{H}_{17} \mathrm{~N}_{3}$ : C, 67.00; H, 9.56; N, 23.44. Found: C, 66.95; H, 9.38; N, 23.37.

\section{(2-Azido-2-methyl-propoxy)-tert-butyl-diphenyl-silane (239)}

$$
{ }_{t-\mathrm{BuPh}_{2} \mathrm{SiO}}^{\mathrm{Me}} \longrightarrow{ }_{223}^{\mathrm{Me}} \longrightarrow \mathrm{BuPh}_{2} \mathrm{SiO} \underbrace{\mathrm{N}_{3}}_{239} \underbrace{\mathrm{Me}}_{\mathrm{Me}}
$$

Following GP5 Azide 239 (130 mg, $0.368 \mathrm{mmol}$, 74\%) and tert-butyl-(2-methyl-allyloxy)-diphenyl-silane (223) (31 mg, 0.10 mmol, 20\% recovery) were obtained as oils with tert-butyl-(2-methyl-allyloxy)-diphenyl-silane (223) (156 mg, $0.502 \mathrm{mmol}, 1.00$ equiv) in $24 \mathrm{~h}$ after purification by column chromatography (solvent gradient: $\mathrm{CH}_{2} \mathrm{Cl}_{2} /$ hexane 1:10, then $\mathrm{CH}_{2} \mathrm{Cl}_{2} / \mathrm{hexane}$ :5). Following GP6 Azide 239 (101 mg, $0.286 \mathrm{mmol}, 58 \%)$ and tert-butyl-(2-methyl-allyloxy)-diphenyl-silane (223) (50 mg, 0.16 mmol, 33\% recovery) were obtained as oils with tert-butyl-(2-methyl-allyloxy)-diphenyl-silane (223) (152 $\mathrm{mg}, 0.490 \mathrm{mmol}, 1.00$ equiv) and TMDSO (0.26 mL, $1.5 \mathrm{mmol}, 3.0$ equiv) in $24 \mathrm{~h}$ after purification by column chromatography (solvent gradient: $\mathrm{CH}_{2} \mathrm{Cl}_{2}$ /hexane 1:10, then $\mathrm{CH}_{2} \mathrm{Cl}_{2}$ /hexane 1:5). Following GP7 Azide 239 (155 mg, $0.439 \mathrm{mmol}, 89 \%$ ) was obtained as an oil with tert-butyl-(2-methyl-allyloxy)-diphenyl-silane (223) (154 mg, $0.496 \mathrm{mmol}, 1.00$ equiv) in $20 \mathrm{~h}$ after purification by column chromatography (solvent gradient: $\mathrm{CH}_{2} \mathrm{Cl}_{2}$ /hexane 1:10, then $\mathrm{CH}_{2} \mathrm{Cl}_{2} /$ hexane 1:5). Following GP8 Azide 239 (162 mg, 0.458 mmol, 91\%) was obtained as an oil with tert-butyl-(2-methyl-allyloxy)-diphenyl-silane (223) (156 mg, $0.502 \mathrm{mmol}, 1.00$ equiv) in $20 \mathrm{~h}$ after purification by column chromatography (solvent gradient: $\mathrm{CH}_{2} \mathrm{Cl}_{2} /$ hexane 1:10, then $\mathrm{CH}_{2} \mathrm{Cl}_{2} / \mathrm{hexane}$ :5). $\mathrm{R}_{\mathrm{f}}$ (AcOEt/hexane 1:40) 0.30; ${ }^{1} \mathrm{H}$ NMR $\left(\mathrm{CDCl}_{3}, 300 \mathrm{MHz}\right) \delta 7.70-7.66(\mathrm{~m}, 4 \mathrm{H}, \mathrm{Ar} \mathrm{H}), 7.45-7.38(\mathrm{~m}, 6 \mathrm{H}, \mathrm{Ar} \mathrm{H}), 3.51\left(\mathrm{~s}, 2 \mathrm{H}, \mathrm{CH}_{2} \mathrm{O}\right)$,

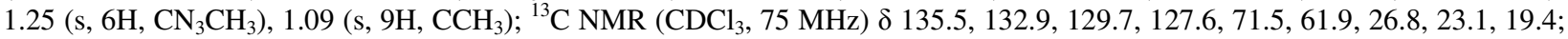
IR v 3070 (w), 3050 (w), 2961 (w), 2931 (m), 2895 (w), 2858 (m), 2134 (m), 2098 (s), 2035 (w), 1890 (w), 1824 (w), 1589 (w), $1472(\mathrm{w}), 1428(\mathrm{~m}), 1392(\mathrm{w}), 1365(\mathrm{w}), 1265(\mathrm{~m}), 1164(\mathrm{w}), 1112(\mathrm{~s}), 1007(\mathrm{w}), 998(\mathrm{w}), 938(\mathrm{w}), 913(\mathrm{w}), 866(\mathrm{w}), 825(\mathrm{~m})$, 773 (w), 741 (m), 702 (s), $614(\mathrm{~m}), 504(\mathrm{~m}), 489(\mathrm{~m})$; MS (ESI) 376.1 (M+Na); Anal. calcd for $\mathrm{C}_{20} \mathrm{H}_{27} \mathrm{~N}_{3} \mathrm{OSi}$ : C, 67.95; H, 7.70; N, 11.89. Found: C, 68.16; H, 7.93; N, 11.82 .

Azidocyclobutane (240) ${ }^{53}$

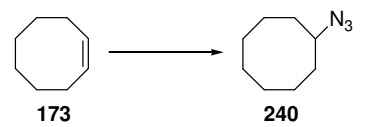

$\mathrm{Co}\left(\mathrm{BF}_{4}\right)_{2} \cdot 6 \mathrm{H}_{2} \mathrm{O}$ (20 mg, $0.060 \mathrm{mmol}, 0.060$ equiv) and ligand 43 (28 mg, $0.060 \mathrm{mmol}, 0.060$ equiv) were dissolved in ethanol $(5 \mathrm{~mL})$ at $23{ }^{\circ} \mathrm{C}$ under argon. After $10 \mathrm{~min}$, cyclooctene $(\mathbf{1 7 3})(0.13 \mathrm{~mL}, 1.0 \mathrm{mmol}, 1.0$ equiv) was added to the homogenous orange solution, followed by tosyl azide $(\mathbf{2 0 3})(0.46 \mathrm{~mL}, 3.0 \mathrm{mmol}, 3.0$ equiv) and tert-butyl hydroperoxide $(5.5 \mathrm{M}$ in decane, 50 $\mu \mathrm{L}, 0.28 \mathrm{mmol}, 0.28$ equiv). After $5 \mathrm{~min}$, phenylsilane $(0.20 \mathrm{~mL}, 1.6 \mathrm{mmol}, 1.6$ equiv) was added dropwise. The resulting brown solution was stirred at $23{ }^{\circ} \mathrm{C}$ and the reaction monitored by TLC (AcOEt/hexane 1:40). After completion (12 h) the reaction was quenched with $\mathrm{H}_{2} \mathrm{O}(5 \mathrm{~mL})$. Sat. $\mathrm{NaHCO}_{3}(2 \mathrm{~mL})$ and brine $(5 \mathrm{~mL})$ were added and the reaction mixture was extracted with $\mathrm{Et}_{2} \mathrm{O}$ $(3 \times 20 \mathrm{~mL})$. The combined organic layers were washed with water $(4 \times 10 \mathrm{~mL})$ and brine $(10 \mathrm{~mL})$, dried over $\mathrm{Na}_{2} \mathrm{SO}_{4}, \mathrm{filtered}$ and the solvents were removed under reduced pressure $\left(p=700 \mathrm{mbar}, T=40{ }^{\circ} \mathrm{C}\right)$. The isolated product was purified by column chromatography (pentane/ $\left.\mathrm{Et}_{2} \mathrm{O} 100: 1\right)$ to afford the desired alkyl azide 240 (86 $\mathrm{mg}, 0.56 \mathrm{mmol}, 56 \%$ ) as a colorless liquid. $\mathrm{R}_{\mathrm{f}}$ (AcOEt/hexane 1:40) 0.50; ${ }^{1} \mathrm{H}$ NMR $\left(\mathrm{CDCl}_{3}, 300 \mathrm{MHz}\right) \delta 3.59-3.52\left(\mathrm{~m}, 1 \mathrm{H}, \mathrm{CHN}_{3}\right), 1.92-1.42\left(\mathrm{~m}, 14 \mathrm{H}, \mathrm{CH}_{2}\right) ;{ }^{13} \mathrm{C} \mathrm{NMR}\left(\mathrm{CDCl}_{3}\right.$, $75 \mathrm{MHz}) \delta$ 62.2, 30.8, 27.2, 25.1, 23.1; IR v 2924 (s), 2855 (m), 2091 (s), 1474 (m), 1447 (m), 1362 (w), 1251 (m), 1095 (w), $1047(w), 940(w), 872(w), 806(w), 668(w)$.

\section{(3-Azido-3-methyl-butoxy)-tert-butyl-diphenyl-silane (241)}

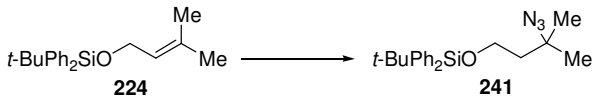


Following GP5 Azide 241 (116 mg, 0.316 mmol, 66\%) and tert-butyl-(3-methyl-but-2-enyloxy)-diphenyl-silane (224) (34 mg, $0.10 \mathrm{mmol}, 21 \%$ recovery) were obtained as oils with tert-butyl-(3-methyl-but-2-enyloxy)-diphenyl-silane (224) (157 mg, 0.484 mmol, 1.00 equiv) in $24 \mathrm{~h}$ after purification by column chromatography (solvent gradient: $\mathrm{CH}_{2} \mathrm{Cl}_{2} / \mathrm{hexane} 1: 10$, then $\mathrm{CH}_{2} \mathrm{Cl}_{2}$ /hexane 1:5). Following GP6 Azide 241 (85 mg, $0.23 \mathrm{mmol}, 50 \%$ ) and tert-butyl-(3-methyl-but-2-enyloxy)-diphenylsilane (224) (74 mg, $0.23 \mathrm{mmol}, 50 \%$ recovery) were obtained as oils with tert-butyl-(3-methyl-but-2-enyloxy)-diphenyl-silane (224) $(150 \mathrm{mg}, 0.462 \mathrm{mmol}, 1.00$ equiv) and TMDSO $(0.26 \mathrm{~mL}, 1.5 \mathrm{mmol}, 3.0$ equiv) in $24 \mathrm{~h}$ after purification by column chromatography (solvent gradient: $\mathrm{CH}_{2} \mathrm{Cl}_{2} /$ hexane 1:10, then $\mathrm{CH}_{2} \mathrm{Cl}_{2} /$ hexane 1:5). Following GP7 Azide 241 (151 mg, 0.411 mmol, 83\%) and tert-butyl-(3-methyl-but-2-enyloxy)-diphenyl-silane (224) $(9 \mathrm{mg}, 0.03 \mathrm{mmol}, 6 \%$ recovery) were obtained as oils with tert-butyl-(3-methyl-but-2-enyloxy)-diphenyl-silane (224) (161 $\mathrm{mg}, 0.496 \mathrm{mmol}, 1.00$ equiv) in $24 \mathrm{~h}$ after purification by column chromatography (solvent gradient: $\mathrm{CH}_{2} \mathrm{Cl}_{2}$ /hexane $1: 10$, then $\mathrm{CH}_{2} \mathrm{Cl}_{2} /$ hexane 1:5). Following GP8 Azide 241 (142 $\mathrm{mg}, 0.386 \mathrm{mmol}, 79 \%)$ was obtained as an oil with tert-butyl-(3-methyl-but-2-enyloxy)-diphenyl-silane (224) (158 mg, 0.487 mmol, 1.00 equiv) in $24 \mathrm{~h}$ after purification by column chromatography (solvent gradient: $\mathrm{CH}_{2} \mathrm{Cl}_{2} / \mathrm{hexane} 1: 10$, then $\mathrm{CH}_{2} \mathrm{Cl}_{2}$ /hexane 1:5). $\mathrm{R}_{\mathrm{f}}$ (AcOEt/hexane 1:40) $0.35 ;{ }^{1} \mathrm{H} \mathrm{NMR}\left(\mathrm{CDCl}_{3}, 300 \mathrm{MHz}\right) \delta 7.70-7.67(\mathrm{~m}, 4 \mathrm{H}, \mathrm{Ar} \mathrm{H}), 7.45-7.37(\mathrm{~m}, 6 \mathrm{H}, \mathrm{Ar}$ $\mathrm{H}), 3.79\left(\mathrm{t}, J=6.8 \mathrm{~Hz}, 2 \mathrm{H}, \mathrm{CH}_{2} \mathrm{O}\right), 1.81\left(\mathrm{t}, J=6.8 \mathrm{~Hz}, 2 \mathrm{H}, \mathrm{CN}_{3} \mathrm{CH}_{2}\right), 1.28\left(\mathrm{~s}, 6 \mathrm{H}, \mathrm{CN}_{3} \mathrm{CH}_{3}\right), 1.06\left(\mathrm{~s}, 9 \mathrm{H}, \mathrm{CCH}_{3}\right) ;{ }^{13} \mathrm{C} \mathrm{NMR}$ $\left(\mathrm{CDCl}_{3}, 75 \mathrm{MHz}\right) \delta 135.4,133.5,129.6,127.6,60.7,60.3,43.5,26.9,26.6,19.2 ; \mathrm{IR} v 3070(\mathrm{w}), 3050(\mathrm{w}), 2962(\mathrm{~m}), 2931(\mathrm{~m})$, 2887 (m), 2857 (m), 2099 (s), 1889 (w), 1824 (w), 1652 (w), 1589 (w), 1472 (w), 1428 (m), 1389 (w), 1370 (w), 1304 (w), 1260 (m), $1186(\mathrm{w}), 1144$ (w), 1112 (s), 1048 (w), 997 (w), 938 (w), 913 (w), 893 (w), 823 (w), 758 (w), 738 (m), 702 (s), 689 (w), 613 $(\mathrm{m}), 505(\mathrm{~m}), 490(\mathrm{~m}), 416(\mathrm{~s})$; MS (ESI) $362.1\left(\mathrm{M}-\mathrm{N}_{2}+\mathrm{Na}\right), 390.0(\mathrm{M}+\mathrm{Na})$; Anal. calcd for $\mathrm{C}_{21} \mathrm{H}_{29} \mathrm{~N}_{3} \mathrm{OSi}$ : C, 68.62; H, 7.95; N, 11.43. Found: C, $68.46 ; \mathrm{H}, 7.74 ; \mathrm{N}, 11.28$.

(3-Azido-3-methyl-pentyl)-benzene (242)

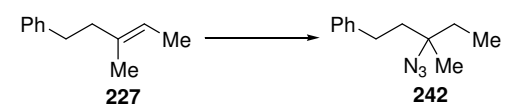

Following GP5 (slightly modified) $\mathrm{Co}\left(\mathrm{BF}_{4}\right)_{2} \bullet 6 \mathrm{H}_{2} \mathrm{O}(6 \mathrm{mg}, 0.02 \mathrm{mmol}, 0.06$ equiv) and ligand $\mathbf{4 3}(8 \mathrm{mg}, 0.02 \mathrm{mmol}, 0.06$ equiv) were dissolved in ethanol $(1.5 \mathrm{~mL})$ at $23{ }^{\circ} \mathrm{C}$ under argon. After $10 \mathrm{~min},((E)-3$-methyl-pent-3-enyl)-benzene (227) (46 mg, $0.29 \mathrm{mmol}, 1.0$ equiv) was added to the homogenous orange solution, followed by tosylazide $(\mathbf{2 0 3})(0.14 \mathrm{~mL}, 0.90 \mathrm{mmol}, 3.0$ equiv) and tert-butyl hydroperoxide $(5.5 \mathrm{M}$ in decane, $15 \mu \mathrm{L}, 0.083 \mathrm{mmol}, 0.28$ equiv). After 5 min phenylsilane $(60 \mu \mathrm{L}, 0.48$ mmol, 1.6 equiv) was added dropwise. The resulting brown solution was stirred at $23{ }^{\circ} \mathrm{C}$ and the reaction monitored by TLC (AcOEt/hexane 1:40). After $30 \mathrm{~h}$ the reaction was quenched with $\mathrm{H}_{2} \mathrm{O}(2 \mathrm{~mL})$. Sat. $\mathrm{NaHCO}_{3}(2 \mathrm{~mL})$ and brine $(5 \mathrm{~mL})$ were added and the reaction mixture was extracted with AcOEt $(3 \times 10 \mathrm{~mL})$. The combined organic layers were dried over $\mathrm{Na}_{2} \mathrm{SO}_{4}$, filtered and the solvents were removed under reduced pressure $\left(p=50 \mathrm{mbar}, T=40{ }^{\circ} \mathrm{C}\right)$. The isolated product was purified by column chromatography (solvent gradient: pentane, then pentane/Et $\mathrm{H}_{2} \mathrm{O}$ 60:1) to afford $\mathbf{2 4 2}$ (39 $\left.\mathrm{mg}, 0.19 \mathrm{mmol}, 66 \%\right)$ as a colorless liquid. Following GP11 (slightly modified) $\mathrm{Co}\left(\mathrm{BF}_{4}\right)_{2} \bullet 6 \mathrm{H}_{2} \mathrm{O}(6 \mathrm{mg}, 0.02 \mathrm{mmol}, 0.06$ equiv) and ligand $\mathbf{4 3}$ (8 $\mathrm{mg}, 0.02 \mathrm{mmol}, 0.06 \mathrm{equiv})$ were dissolved in ethanol $(1.5 \mathrm{~mL})$ at $23{ }^{\circ} \mathrm{C}$ under argon. After $10 \mathrm{~min},((E)$-3-methyl-pent-3-enyl)-benzene (227) (46 mg, 0.29 mmol, 1.0 equiv) was added to the homogenous orange solution, followed by tosylazide $(\mathbf{2 0 3})(0.14 \mathrm{~mL}, 0.90 \mathrm{mmol}, 3.0 \mathrm{equiv})$ and tert-butyl hydroperoxide (5.5 $\mathrm{M}$ in decane, $15 \mu \mathrm{L}, 0.083 \mathrm{mmol}, 0.28$ equiv). After $5 \mathrm{~min}$ TMDSO (0.16 mL, $0.90 \mathrm{mmol}, 3.0$ equiv) was added dropwise. The resulting brown solution was stirred at $23{ }^{\circ} \mathrm{C}$ and the reaction monitored by TLC (AcOEt/hexane 1:40). After $30 \mathrm{~h}$ the reaction was quenched with $\mathrm{H}_{2} \mathrm{O}(2 \mathrm{~mL})$. Sat. $\mathrm{NaHCO}_{3}(2 \mathrm{~mL})$ and brine $(5 \mathrm{~mL})$ were added and the reaction mixture was extracted with AcOEt $(3 \times 10 \mathrm{~mL})$. The combined organic layers were dried over $\mathrm{Na}_{2} \mathrm{SO}_{4}$, filtered and the solvents were removed under reduced pressure $\left(p=50 \mathrm{mbar}, T=40^{\circ} \mathrm{C}\right)$. The isolated product was purified by column chromatography (solvent gradient: pentane, then pentane/ $\mathrm{Et}_{2} \mathrm{O}$ 60:1) to afford $242\left(28 \mathrm{mg}, 0.14 \mathrm{mmol}, 48 \%\right.$ ) as a colorless liquid. $\mathrm{R}_{\mathrm{f}}$ (AcOEt/hexane 1:40) 0.30; ${ }^{1} \mathrm{H}$ NMR $\left(\mathrm{CDCl}_{3}, 300 \mathrm{MHz}\right) \delta 7.33-7.18(\mathrm{~m}, 5 \mathrm{H}, \mathrm{Ar} \mathrm{H}), 2.69-2.63\left(\mathrm{~m}, 2 \mathrm{H}, \mathrm{PhCH}_{2}\right), 1.83-1.76(\mathrm{~m}, 2 \mathrm{H}$, $\left.\mathrm{CH}_{2} \mathrm{CN}_{3}\right), 1.63\left(\mathrm{q}, J=7.5 \mathrm{~Hz}, 2 \mathrm{H}, \mathrm{CH}_{2} \mathrm{CH}_{3}\right), 1.30\left(\mathrm{~s}, 3 \mathrm{H}, \mathrm{CN}_{3} \mathrm{CH}_{3}\right), 0.97\left(\mathrm{t}, J=7.5 \mathrm{~Hz}, 3 \mathrm{H}, \mathrm{CH}_{2} \mathrm{CH}_{3}\right) ;{ }^{13} \mathrm{C} \mathrm{NMR}\left(\mathrm{CDCl}_{3}, 75\right.$ MHz) $\delta 141.8,128.4,128.2,125.8,64.3,41.1,32.1,30.5,22.9,8.5 ;$ IR $v 3063(w), 3026(w), 2971(w), 2939(w), 2880(w)$, $2092(\mathrm{~s}), 1603(\mathrm{w}), 1497(\mathrm{w}), 1455(\mathrm{w}), 1381(\mathrm{w}), 1256(\mathrm{~m}), 1190(\mathrm{w}), 1136(\mathrm{w}), 1074(\mathrm{w}), 1030(\mathrm{w}), 912(\mathrm{w}), 856(\mathrm{w}), 741(\mathrm{~m})$, $698(\mathrm{~m}), 565(\mathrm{w}), 510(\mathrm{w}), 460(\mathrm{w}), 450(\mathrm{w})$; MS (EI-HIRES) 160.1171 (M-HN $)_{3}, 174.1281\left(\mathrm{M}-\mathrm{HN}_{2}\right), 175.1343\left(\mathrm{M}-\mathrm{N}_{2}\right)$; Anal. calcd for $\mathrm{C}_{12} \mathrm{H}_{17} \mathrm{~N}_{3}$ : C, 70.90; H, 8.43; N, 20.67. Found: C, 71.15; H, 8.52; N, 20.76.

(2-Azido-2-methyl-propoxymethyl)-benzene (243)

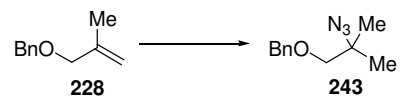

Following GP6 Azide 243 (41 mg, $0.20 \mathrm{mmol}, 40 \%$ ) and alkene 228 (22 mg, $0.13 \mathrm{mmol}, 26 \%$ recovery) were obtained as oils with alkene 228 ( $81 \mathrm{mg}, 0.50 \mathrm{mmol}, 1.0$ equiv) and TMDSO (0.18 mL, $1.0 \mathrm{mmol}, 2.0$ equiv) in $24 \mathrm{~h}$ after purification by column chromatography (pentane/Et ${ }_{2} \mathrm{O}$ 60:1). Following GP7 Azide 243 (66 mg, $0.32 \mathrm{mmol}, 64 \%$ ) was obtained as an oil with alkene 228 ( $81 \mathrm{mg}, 0.50 \mathrm{mmol}, 1.0$ equiv) in $24 \mathrm{~h}$ after purification by column chromatography (solvent gradient: pentane/Et $\mathrm{O}_{2} \mathrm{O} 80: 1$, then pentane/ $\mathrm{Et}_{2} \mathrm{O}$ 40:1). Following GP8Azide $243(79 \mathrm{mg}, 0.38 \mathrm{mmol}, 76 \%)$ was obtained as an oil with alkene $228(81 \mathrm{mg}$, $0.50 \mathrm{mmol}, 1.0$ equiv) in $24 \mathrm{~h}$ after purification by column chromatography (pentane $\left./ \mathrm{Et}_{2} \mathrm{O} 60: 1\right)$. $\mathrm{R}_{\mathrm{f}}($ AcOEt/hexane $1: 40) 0.30$; ${ }^{1} \mathrm{H}$ NMR $\left(\mathrm{CDCl}_{3}, 300 \mathrm{MHz}\right) \delta$ 7.39-7.30 (m, 5H, Ar H), $4.60\left(\mathrm{~s}, 2 \mathrm{H}, \mathrm{PhCH}_{2} \mathrm{O}\right), 3.38\left(\mathrm{~s}, 2 \mathrm{H}, \mathrm{CH}_{2} \mathrm{OBn}, 1.30\left(\mathrm{~s}, 6 \mathrm{H}, \mathrm{CH}_{3}\right) ;{ }^{13} \mathrm{C} \mathrm{NMR}\right.$ $\left(\mathrm{CDCl}_{3}, 75 \mathrm{MHz}\right) \delta 137.9,128.2,127.5,127.3,77.3,73.3,61.0,23.5$; IR v $3090(\mathrm{w}), 3066(\mathrm{w}), 3031(\mathrm{w}), 2977(\mathrm{~m}), 2935(\mathrm{w})$, $2861(\mathrm{~m}), 2502(\mathrm{w}), 2100(\mathrm{~s}), 2043(\mathrm{w}), 1587(\mathrm{w}), 1497(\mathrm{w}), 1470(\mathrm{w}), 1454(\mathrm{~m}), 1410(\mathrm{w}), 1384(\mathrm{w}), 1368(\mathrm{~m}), 1265(\mathrm{~s}), 1208$ 
(w), 1164 (w), 1104 (s), 1029 (m), $981(w), 960$ (w), 906 (w), $870(w), 783(w), 737$ (m), 698 (m), 620 (w), 605 (w), 562 (w); MS (ESI) $228.0\left(\mathrm{M}+\mathrm{Na}\right.$ );Anal. calcd for $\mathrm{C}_{11} \mathrm{H}_{15} \mathrm{~N}_{3} \mathrm{O}$ : C, 64.37; H, 7.37; N, 20.47. Found: C, 64.55; H, 7.47; N, 20.36.

\section{Mechanistic Investigations}

4.1. NMR and Deuterium-Labeling Experiments

NMR Experiments

\section{Stoichiometric Reaction with Complex 20}

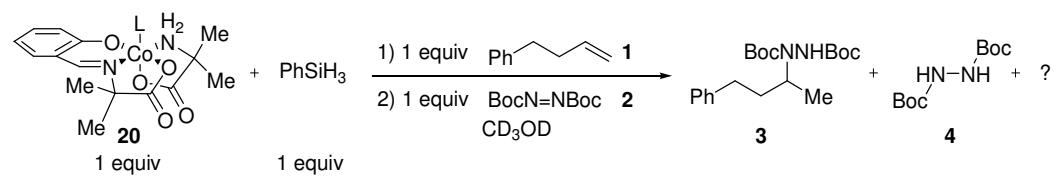

Complex 20 (10 mg, $0.025 \mathrm{mmol}, 1.0$ equiv) was dissolved in $\mathrm{CD}_{3} \mathrm{OD}(0.75 \mathrm{~mL})$ at $23{ }^{\circ} \mathrm{C}$ under argon and the first ${ }^{1} H$-NMR (300 MHz) spectrum A was measured. Phenylsilane $\left(3.1 \mu \mathrm{L}, 0.025 \mathrm{mmol}, 1.0\right.$ equiv) was added and the second ${ }^{1} H$-NMR spectrum $\mathbf{B}$ was measured as fast as possible, showing no peaks anymore corresponding to $\mathbf{2 0}$. The reaction mixture was then heated progressively to $40{ }^{\circ} \mathrm{C}$ and monitored by ${ }^{1} \mathrm{H}$-NMR spectroscopy. After $20 \mathrm{~min}$, at $40{ }^{\circ} \mathrm{C}$, spectrum $\mathbf{C}$ was measured. At this point, the reaction mixture was cooled to $23{ }^{\circ} \mathrm{C}$ and 4-phenylbutene (1) $(3.8 \mu \mathrm{L}, 0.025 \mathrm{mmol}, 1.0$ equiv) was added and the spectrum $\mathbf{D}$ was measured. Finally, after $5 \mathrm{~min}$, azodicarboxylate $2(6.0 \mathrm{mg}, 0.026 \mathrm{mmol}, 1.0$ equiv) was added and the reaction monitored via ${ }^{1} \mathrm{H}$-NMR spectroscopy for the following $30 \mathrm{~min}$. After $30 \mathrm{~min}$, spectrum $\mathbf{E}$ was obtained and no major changes were observed afterwards.

\section{Deuterium-Labeling Experiments}

$N$-(3-Phenyl-1-deuteromethyl-propyl)-N'-(tert-butoxycarbonyl)hydrazinecarboxylic acid tert-butyl ester (48)

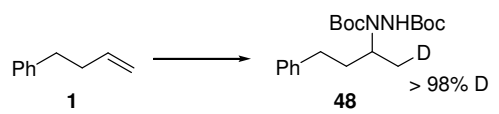

Following GP2 (with $\mathbf{P h S i D}_{3}$ ) Hydrohydrazination product 48 (132 mg, $\left.0.361 \mathrm{mmol}, 72 \%\right)$ was obtained as a colorless solid with 4-phenylbutene (1) $\left(75 \mu \mathrm{L}, 0.50 \mathrm{mmol}, 1.0\right.$ equiv) and $\mathrm{PhSiD}_{3}$ (synthesized following a reported procedure, ${ }^{54}>98 \% \mathrm{D}, 82 \%$ in $\mathrm{Et}_{2} \mathrm{O}, 70 \mathrm{mg}, 0.52 \mathrm{mmol}, 1.0$ equiv) in $10 \mathrm{~h}$ with $5 \mathrm{~mol} \%$ catalyst 20 after purification by column chromatography (AcOEt/hexane 1:10). $\mathrm{R}_{\mathrm{f}}\left(\mathrm{AcOEt} /\right.$ hexane 1:5) 0.35; Mp 127-128 ${ }^{\circ} \mathrm{C} ;{ }^{1} \mathrm{H}$ NMR $\left(\mathrm{CDCl}_{3}, 300 \mathrm{MHz}, 52{ }^{\circ} \mathrm{C}\right) \delta 7.29-7.14(\mathrm{~m}, 5 \mathrm{H}, \mathrm{Ar}$ $\mathrm{H}), 5.86$ (br s, $1 \mathrm{H}, \mathrm{NH}), 4.24$ (br s, $1 \mathrm{H}, \mathrm{CHN}), 2.65\left(\mathrm{~m}, 2 \mathrm{H}, \mathrm{PhCH}_{2}\right), 1.88\left(\mathrm{~m}, 1 \mathrm{H}, \mathrm{CH}_{2} \mathrm{CHN}\right), 1.65\left(\mathrm{~m}, 1 \mathrm{H}, \mathrm{CH} \mathrm{CHN}_{2}\right), 1.48(\mathrm{~s}$, $\left.9 \mathrm{H}, \mathrm{CCH}_{3}\right), 1.47\left(\mathrm{~s}, 9 \mathrm{H}, \mathrm{CCH}_{3}\right), 1.12\left(\mathrm{~d}, J=6.5 \mathrm{~Hz}, 2 \mathrm{H}, \mathrm{CHNCH}_{2} \mathrm{D}\right) ;{ }^{13} \mathrm{C} \mathrm{NMR}\left(\mathrm{CDCl}_{3}, 75 \mathrm{MHz}, 52{ }^{\circ} \mathrm{C}\right) \delta 156.0,155.0,142.1$, 128.3, 128.3, 125.7, 80.9, 53.0, 35.8, 32.9, 28.3, 28.2, 17.8 (t, J = 19 Hz); IR v 3269 (m), 3159 (w), 3085 (w), $3062(w), 3026(w)$, 2977 (s), 2932 (m), $2864(\mathrm{w}), 2252(\mathrm{w}), 2185(\mathrm{w}), 1945(\mathrm{w}), 1746(\mathrm{~s}), 1703(\mathrm{~s}), 1604(\mathrm{w}), 1495(\mathrm{~m}), 1478(\mathrm{~m}), 1454(\mathrm{~m}), 1392$ (s), 1366 (s), 1273 (s), 1249 (s), 1159 (s), 1099 (m), 1079 (m), 1052 (m), 1032 (m), 1015 (m), 911 (m), 859 (m), 748 (m), 755 $(\mathrm{m}), 700(\mathrm{~m}), 647(\mathrm{w})$; MS (ESI) $366.4(\mathrm{M}+\mathrm{H}), 388.2(\mathrm{M}+\mathrm{Na})$; Anal. calcd for $\mathrm{C}_{20} \mathrm{H}_{31} \mathrm{DN}_{2} \mathrm{O}_{4}$ : C, 65.73; H+D, 8.85; N, 7.66. Found: C, 65.99; H+D, 8.93; N, 7.58.

\section{$N$-(2-Deutero-1H-inden-1-yl)- $N^{\prime}$-(tert-butoxycarbonyl)hydrazinecarboxylic acid tert-butyl ester (50)}

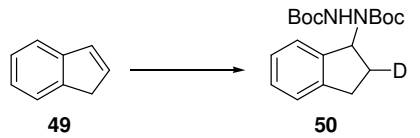

Following GP1 (with $\mathbf{P h S i D}_{3}$ ) Hydrohydrazination product 50 (1:1 mixture of diastereoisomers, $154 \mathrm{mg}, 0.441 \mathrm{mmol}, 88 \%$ ) was obtained as a colorless solid with indene (49) $\left(59 \mu \mathrm{L}, 0.50 \mathrm{mmol}, 1.0\right.$ equiv) and $\mathrm{PhSiD}_{3}$ (synthesized following a reported procedure, ${ }^{54}>98 \% \mathrm{D}, 82 \%$ in $\mathrm{Et}_{2} \mathrm{O}, 70 \mathrm{mg}, 0.52 \mathrm{mmol}, 1.0$ equiv) in $5 \mathrm{~h}$ with $5 \mathrm{~mol} \%$ catalyst 20 after purification by column chromatography (AcOEt/hexane 1:10). $\mathrm{R}_{\mathrm{f}}\left(\mathrm{AcOEt} /\right.$ hexane 1:5) $0.35 ; \mathrm{Mp} 95-97{ }^{\circ} \mathrm{C} ;{ }^{1} \mathrm{H} \mathrm{NMR}\left(\mathrm{CDCl}_{3}, 300 \mathrm{MHz}, 52{ }^{\circ} \mathrm{C}\right) \delta 7.23-$ $7.15(\mathrm{~m}, 4 \mathrm{H}, \mathrm{Ar} \mathrm{H}), 5.88$ (br s, $1 \mathrm{H}, \mathrm{NH}), 5.75(\mathrm{br} \mathrm{s}, 1 \mathrm{H}, \mathrm{CHN}), 2.99\left(\mathrm{~m}, 1 \mathrm{H}, \mathrm{CH}_{2}\right), 2.81\left(\mathrm{dd}, J=15.9,8.0 \mathrm{~Hz}, 1 \mathrm{H}, \mathrm{CH}_{2}\right), 2.37(\mathrm{~m}$,

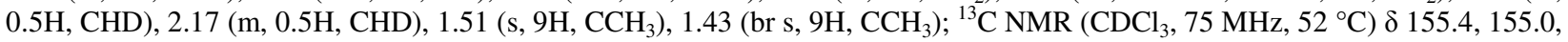
144.0, 141.1, 127.8, 126.4, 124.8, 124.1, 81.2, 80.8, 62.4, 30.3, $28.8(\mathrm{~m}), 28.2,28.1$; IR v $3313(\mathrm{~m}), 3071(\mathrm{w}), 2977(\mathrm{~s}), 2932(\mathrm{~m})$, $2855(\mathrm{w}), 2252(\mathrm{w}), 2201(\mathrm{w}), 1742(\mathrm{~s}), 1704(\mathrm{~s}), 1606(\mathrm{w}), 1479(\mathrm{~m}), 1458(\mathrm{~m}), 1392(\mathrm{~s}), 1367$ (s), 1327 (s), 1254 (s), 1158 (s), $1100(\mathrm{~m}), 1052(\mathrm{~m}), 1023(\mathrm{~m}), 987(\mathrm{w}), 940(\mathrm{w}), 918(\mathrm{w}), 874(\mathrm{w}), 854(\mathrm{w}), 750(\mathrm{~s}), 700(\mathrm{w}), 647(\mathrm{w})$; MS (ESI) 350.2 (M+H), $372.2(\mathrm{M}+\mathrm{Na})$; Anal. calcd for $\mathrm{C}_{19} \mathrm{H}_{27} \mathrm{DN}_{2} \mathrm{O}_{4}: \mathrm{C}, 65.31 ; \mathrm{H}+\mathrm{D}, 8.10 ; \mathrm{N}, 8.02$. Found: C, 65.28; H, 8.10; N, 7.85.

Di-tert-butyl-1-(8-deutero-cyclooct-2-enyl)hydrazine-1,2-dicarboxylate (53a) and Di-tert-butyl-1-(4-deutero-cyclooct-2enyl)hydrazine-1,2-dicarboxylate (53b)

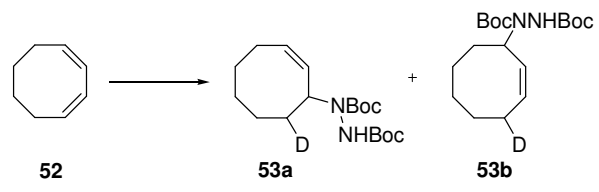


Following GP1 (with $\mathbf{P h S i D}_{\mathbf{3}}$ ) Hydrohydrazination product $\mathbf{5 3}$ (1:1 mixture of regioisomers 53a, 53b, each regioisomer 1:1 mixture of diastereoisomers, $88 \mathrm{mg}, 0.26 \mathrm{mmol}, 52 \%)$ was obtained as a colorless solid with cyclooctadiene $(\mathbf{5 2})(62 \mu \mathrm{L}, 0.50$ mmol, 1.0 equiv) and $\mathrm{PhSiD}_{3}$ (synthesized following a reported procedure, ${ }^{54}>98 \% \mathrm{D}, 82 \%$ in $\mathrm{Et}_{2} \mathrm{O}, 70 \mathrm{mg}, 0.52 \mathrm{mmol}, 1.0$ equiv) in $6 \mathrm{~h}$ with $5 \mathrm{~mol} \%$ catalyst 20 after purification by column chromatography (AcOEt/hexane 1:10). $\mathrm{R}_{\mathrm{f}}(\mathrm{AcOEt} / \mathrm{hexane}$ 1:10) 0.25; Mp 121-123 ${ }^{\circ} \mathrm{C} ;{ }^{1} \mathrm{H}$ NMR $\left(\mathrm{CDCl}_{3}, 300 \mathrm{MHz}, 50{ }^{\circ} \mathrm{C}\right) \delta 6.09$ (br s, $\left.1 \mathrm{H}, \mathrm{NH}\right), 5.70-5.52(\mathrm{~m}, 2 \mathrm{H}, \mathrm{C}=\mathrm{CH}), 5.02(\mathrm{~m}, 1 \mathrm{H}$, $\mathrm{CHN}), 2.30-2.19\left(\mathrm{~m}, 0.7-0.8 \mathrm{H}, \mathrm{CH}_{2} \mathrm{C}=\right.$ and $\left.\mathrm{CHD}=\right), 2.13-2.01\left(\mathrm{~m}, 0.7-0.8 \mathrm{H}, \mathrm{CH}_{2} \mathrm{C}=\right.$ and $\left.\mathrm{CHD}=\right), 1.68-1.24(\mathrm{~m}, 7.5-8 \mathrm{H}), 1.46(\mathrm{~s}$, $\left.9 \mathrm{H}, \mathrm{C}\left(\mathrm{CH}_{3}\right)_{3}\right), 1.45\left(\mathrm{~s}, 9 \mathrm{H}, \mathrm{C}\left(\mathrm{CH}_{3}\right)_{3}\right) ;{ }^{13} \mathrm{C} \mathrm{NMR}\left(\mathrm{CDCl}_{3}, 75 \mathrm{MHz}, 52{ }^{\circ} \mathrm{C}\right) \delta 155.9,154.5,129.3,128.7,81.0,55.4,33.3,33.0(\mathrm{~m})$, 32.7 (m), 29.3, $29.2(\mathrm{~m}), 28.4,28.3,26.8,26.6,24.5,24.4 ;{ }^{2} \mathrm{D}$ NMR $\left(\mathrm{CHCl}_{3}, 300 \mathrm{MHz}, 23{ }^{\circ} \mathrm{C}\right) \delta 2.24(\mathrm{~s}, 1 \mathrm{D}, \mathrm{CHD}=, \mathbf{5 3 b}), 2.09$ (s, 1D, CHD=, 53b), 1.75 (s, 1D, CHDCHN, 53a), 1.58 (s, 1D, CHDCHN, 53a); IR v 3319 (w), 2978 (m), 2929 (m), 2858 (w), 2157 (w), 1704 (s), 1479 (m), 1455 (m), 1412 (m), 1391 (s), 1367 (s), 1315 (m), 1246 (m), 1161 (s), 1050 (w), 1022 (w), 937 (w), 859 (w), 759 (w); MS (ESI) $342.2(\mathrm{M}+\mathrm{H}), 364.2(\mathrm{M}+\mathrm{Na}), 705.2(2 \mathrm{M}+\mathrm{Na})$; Anal. calcd for $\mathrm{C}_{18} \mathrm{H}_{31} \mathrm{DN}_{2} \mathrm{O}_{4}: \mathrm{C}, 63.31 ; \mathrm{H}+\mathrm{D}, 9.47$; $\mathrm{N}, 8.20$. Found: C, 63.35; H+D, 9.52; N, 8.22.

(3-Azido-4-deutero-butyl)-benzene (51)

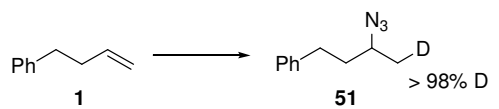

Following GP10 (with $\mathbf{P h S i D}_{3}$ ) Azide 51 (83 mg, $0.47 \mathrm{mmol}$, 94\%) was obtained as a colorless liquid with 4-phenylbutene (1) (75 $\mu \mathrm{L}, 0.50 \mathrm{mmol}, 1.0$ equiv) and $\mathrm{PhSiD}_{3}$ (synthesized following a reported procedure, ${ }^{54}>98 \% \mathrm{D}, 82 \%$ in $\mathrm{Et}_{2} \mathrm{O}, 0.10 \mathrm{~g}, 0.74$ mmol, 1.5 equiv) in $3 \mathrm{~h}$ after purification by column chromatography (solvent gradient: pentane, then pentane/Et ${ }_{2} \mathrm{O} 60: 1$ ). $\mathrm{R}_{\mathrm{f}}$ (AcOEt/hexane 1:40) 0.30; ${ }^{1} \mathrm{H} \mathrm{NMR}\left(\mathrm{CDCl}_{3}, 300 \mathrm{MHz}\right) \delta 7.33-7.18(\mathrm{~m}, 5 \mathrm{H}, \mathrm{Ar} \mathrm{H}), 3.43$ (qi, $J=6.9 \mathrm{~Hz}, 1 \mathrm{H}, \mathrm{CHN}_{3}$ ), $2.81-2.62$ $\left(\mathrm{m}, 2 \mathrm{H}, \mathrm{PhCH}_{2}\right), 1.90-1.72\left(\mathrm{~m}, 2 \mathrm{H}, \mathrm{CH}_{2} \mathrm{CHN}_{3}\right), 1.28\left(\mathrm{dt}, J=6.5 \mathrm{~Hz}, 1.9 \mathrm{~Hz}, 2 \mathrm{H}, \mathrm{CHNCH}_{2} \mathrm{D}\right) ;{ }^{13} \mathrm{C} \mathrm{NMR}\left(\mathrm{CDCl}_{3}, 75 \mathrm{MHz}^{\mathrm{N}} \delta\right.$ 141.1, 128.3, 128.3, 125.9, 57.1, 37.9, 32.4, 19.3 (t, $J=19$ Hz); IR v 3063 (w), 3027 (w), 2941 (m), 2860 (w), $2100(\mathrm{~s}), 1603$ (w), 1496 (w), 1454 (w), 1333 (w), 1261 (m), 1030 (w), 745 (w), 699 (m); HRMS (EI) calcd for $\left.\mathrm{C}_{10} \mathrm{H}_{11} \mathrm{DN}^{+}\left(\mathrm{M}^{-\mathrm{HN}}\right)_{2}\right)$ : 147.1027, found 147.1029; Anal. calcd for $\mathrm{C}_{10} \mathrm{H}_{12} \mathrm{DN}_{3}$ : C, 68.15; H+D, 7.48; N, 23.84. Found: C, 68.15; H+D, 7.70; N, 23.93.

\section{2. $\quad$ Radical Clocks}

( $N$-Pent-3-enyl)- $N$ '-(tert-butoxycarbonyl)hydrazinecarboxylic acid tert-butyl ester (245)

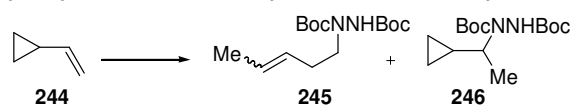

Following GP1 Hydrohydrazination product 245 (80 mg, $0.27 \mathrm{mmol}, 59 \%$, mixture of Z/E olefins (1/5, not assigned), containing traces $(<5 \%)$ of $\mathbf{2 4 6}$ (not isolated)) was obtained as a colorless solid with vinylcyclopropane (244) (synthesized following a reported procedure, ${ }^{55} 93 \%$ pure, $33 \mathrm{mg}, 0.45 \mathrm{mmol}, 1.0 \mathrm{eq}$ ) in $5 \mathrm{~h}$ with $5 \mathrm{~mol} \%$ catalyst 20 after purification by column chromatography (AcOEt/hexane 1:15-1:2). $\mathrm{R}_{\mathrm{f}}$ (AcOEt/hexane 1:5) 0.35; Mp 51-55 ${ }^{\circ} \mathrm{C} ;{ }^{1} \mathrm{H}$ NMR $\left(\mathrm{CDCl}_{3}, 300 \mathrm{MHz}, 52\right.$ $\left.{ }^{\circ} \mathrm{C}\right) \delta 6.24(\mathrm{br} \mathrm{s}, 1 \mathrm{H}, \mathrm{NH}), 5.55-5.31(\mathrm{~m}, 2 \mathrm{H}$, alkene $\mathrm{H}), 3.44\left(\mathrm{dd}, J=6.9,7.5 \mathrm{~Hz}, 2 \mathrm{H}, \mathrm{CH}_{2} \mathrm{Ph}\right), 2.35-2.19(\mathrm{~m}, 2 \mathrm{H}$, alkene-CH$)$, $1.62\left(\mathrm{dd}, J=5.9,1.2 \mathrm{~Hz}, 3 \mathrm{H}(83 \%)\right.$, alkene- $\mathrm{CH}_{3}, 1$. diastereoisomer), $1.46\left(\mathrm{~s}, 9 \mathrm{H}, \mathrm{CCH}_{3}\right), 1.44\left(\mathrm{~s}, 9 \mathrm{H}, \mathrm{CCH}_{3}\right), 1.19(\mathrm{~d}, J=6.9$ $\mathrm{Hz}$, alkene- $\mathrm{CH}_{3}, 3 \mathrm{H}(17 \%)$, 2. diastereoisomer); ${ }^{13} \mathrm{C} \mathrm{NMR}\left(\mathrm{CDCl}_{3}, 75 \mathrm{MHz}, 52{ }^{\circ} \mathrm{C}\right) \delta 155.0,127.8,126.7,125.8,80.9,80.8$, 80.6, 49.7, 30.9, 28.2, 28.2, 25.4, 17.8, 17.4, 15.1, 12.7; IR v $3318(\mathrm{~m}), 2980(\mathrm{~s}), 2934$ (m), $1706(\mathrm{~s}), 1480(\mathrm{~m}), 1455(\mathrm{~m}), 1393$ (s), 1367 (s), 1290 (s), 1252 (s), 1161 (s), 1051 (m), 1019 (m), 967 (w), 932 (w), 857 (w), 758 (m), 701 (w), 605 (w); MS(ESI) $301.2(\mathrm{M}+\mathrm{H}), 323.2(\mathrm{M}+\mathrm{Na})$; Anal. calcd for $\mathrm{C}_{15} \mathrm{H}_{28} \mathrm{~N}_{2} \mathrm{O}_{4}$ : C, 59.98; H, 9.39; N, 9.33. Found: C, 59.77; H, 9.17; N, 9.14.

$\boldsymbol{N}$-(1-Cyclopropyl-1-phenyl-ethyl)- $N$ '-(tert-butoxycarbonyl)hydrazinecarboxylic acid tert-butyl ester (248)

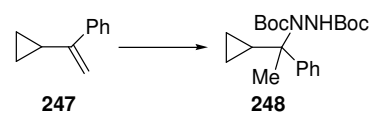

Following GP1 Hydrohydrazination product 248 (91 mg, $0.24 \mathrm{mmol}, 48 \%$ ) was obtained as a colorless solid with vinylcyclopropane 247 (synthesized following a reported procedure, ${ }^{56} 72 \mathrm{mg}, 0.50 \mathrm{mmol}, 1.0 \mathrm{eq}$ ) in $5 \mathrm{~h}$ with $5 \mathrm{~mol} \%$ catalyst $\mathbf{2 0}$ after purification by column chromatography (AcOEt/hexane 1:15-1:2). A fraction containing a mixture of ring-opened and multiaminated products (61 mg, about 20-30\%, exact structure not assigned) was also isolated. Following GP3 Hydrohydrazination product 248 (114 $\mathrm{mg}, 0.303 \mathrm{mmol}, 60 \%$ ) was obtained as a colorless solid with vinylcyclopropane $\mathbf{2 4 7}$ (synthesized following a reported procedure, $\left.{ }^{56} 72 \mathrm{mg}, 0.50 \mathrm{mmol}, 1.0 \mathrm{eq}\right)$ in $3 \mathrm{~h}$ with $2 \mathrm{~mol} \%$ catalyst 23 after purification by column chromatography (AcOEt/hexane 1:15-1:2). A fraction containing a mixture of ring-opened and multi-aminated products (48 mg, about $20 \%$, exact structure not assigned) was also isolated. $\mathrm{R}_{\mathrm{f}}(\mathrm{AcOEt} / \mathrm{h}$ exane $1: 5) 0.30 ; \mathrm{Mp} 53-55{ }^{\circ} \mathrm{C} ;{ }^{1} \mathrm{H} \mathrm{NMR}\left(\mathrm{CDCl}_{3}, 300 \mathrm{MHz}, 52{ }^{\circ} \mathrm{C}\right) \delta 7.56$ (br m, 2H, Ar H), 7.29-7.14 (m, 3H, Ar H), 6.40 (br s, 1H, NH), 1.65-1.50 (m, 1H, CH), $1.53\left(\mathrm{~s}, 9 \mathrm{H}, \mathrm{OC}\left(\mathrm{CH}_{3}\right)_{3}\right), 1.39(\mathrm{~s}, 3 \mathrm{H}$, $\left.\mathrm{CNCH}_{3}\right), 1.15\left(\mathrm{~s}, 9 \mathrm{H}, \mathrm{OC}\left(\mathrm{CH}_{3}\right)_{3}\right), 0.62-0.08\left(\mathrm{~m}, 4 \mathrm{H}, \mathrm{CH}_{2}\right) ;{ }^{13} \mathrm{C} \mathrm{NMR}\left(\mathrm{CDCl}_{3}, 75 \mathrm{MHz}, 52{ }^{\circ} \mathrm{C}\right.$, mixture of rotamers) $\delta 156.3$, 154.5, 147.9, 146.0, 128.5, 127.6, 127.4, 125.8, 125.3, 81.0, 67.7, 67.1, 28.4, 28.0, 23.2, 21.7, 21.0, 19.1, 2.6, 2.4, 0.6; IR v 3270 $(\mathrm{m}), 3086(\mathrm{w}), 3061(\mathrm{w}), 3006(\mathrm{~m}), 2978(\mathrm{~s}), 2932(\mathrm{~m}), 1952(\mathrm{w}), 1711(\mathrm{~s}), 1602(\mathrm{w}), 1493(\mathrm{~m}), 1478(\mathrm{~m}), 1455(\mathrm{~m}), 1392(\mathrm{~s})$, 1367 (s), 1338 (s), 1250 (s), 1161 (s), 1085 (m), 1073 (m), 1048 (m), 1020 (m), 912 (m), 851 (m), 764 (m), 733 (s), 703 (s), 646 (w), 615 (w); MS(ESI) $399.3(\mathrm{M}+\mathrm{Na})$; Anal. calcd for $\mathrm{C}_{21} \mathrm{H}_{32} \mathrm{~N}_{2} \mathrm{O}_{4}$ : C, 66.99; H, 8.57; N, 7.44. Found: C, 67.01; H, 8.47; N, 7.28. 
$N$-(2-Methyl-cyclopentylmethyl)- $N$ '-(tert-butoxycarbonyl)hydrazinecarboxylic acid tert-butyl ester (250) and $N$-(1Methyl-hex-5-enyl)- $N$ '-(tert-butoxycarbonyl)hydrazinecarboxylic acid tert-butyl ester (251) and $N$-[5-( $N$, $N$ '-Di-tertbutoxycarbonyl-hydrazino)-1-methyl-hexyl]- $N$ '-(tert-butoxycarbonyl)hydrazinecarboxylic acid tert-butyl ester (252)

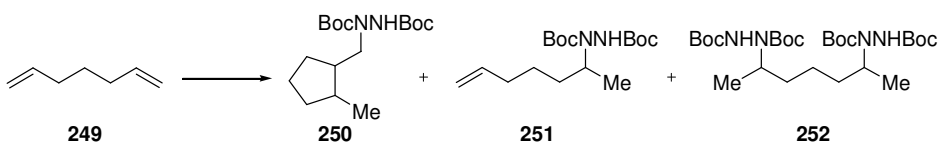

Following GP1 Hydrohydrazination products 251 together with $\mathbf{2 5 0}$ (mixture 251/250 5:1, 250 as a $1.4: 1$ mixture of diastereoisomers, $80 \mathrm{mg}, 0.24 \mathrm{mmol}, 40 \% \mathbf{2 5 1}$ and $8 \% \mathbf{2 5 0})$ and bis-hydrohydrazination product $252(68 \mathrm{mg}, 0.12 \mathrm{mmol}, 24 \%)$ were obtained as a viscous oil (250/251 mixture) and a colorless solid (252) with 1,6-heptadiene 249 (68 $\mu \mathrm{L}, 0.50 \mathrm{mmol}, 1.0 \mathrm{eq})$ in $20 \mathrm{~h}$ with $5 \mathrm{~mol} \%$ catalyst 20 after purification by column chromatography (AcOEt/hexane 1:10-1:1). Following GP3 Hydrohydrazination products $\mathbf{2 5 1}$ together with 250 (mixture 251/250 5:1, 251 as a 5:1 mixture of diastereoisomers, $68 \mathrm{mg}, 0.20$ mmol, 34\% 251 and 6\% 250) and bis-hydrohydrazination product 252 (82 $\mathrm{mg}, 0.15 \mathrm{mmol}, 30 \%)$ were obtained as a viscous oil (250/251 mixture) and a colorless solid (252) with 1,6-heptadiene $\mathbf{2 4 9}$ (68 $\mu \mathrm{L}, 0.50 \mathrm{mmol}, 1.0 \mathrm{eq})$ in $15 \mathrm{~h}$ with 2 mol\% catalyst $\mathbf{2 3}$ after purification by column chromatography (AcOEt/hexane 1:10-1:1).

$N$-(2-Methyl-cyclopentylmethyl)- $N$ '-(tert-butoxycarbonyl)hydrazinecarboxylic acid tert-butyl ester (250) and $N$-(1Methyl-hex-5-enyl)- $N$ '-(tert-butoxycarbonyl)hydrazinecarboxylic acid tert-butyl ester (251)

$\mathrm{R}_{\mathrm{f}}\left(\right.$ AcOEt/hexane 1:5) 0.30; ${ }^{1} \mathrm{H} \mathrm{NMR}\left(\mathrm{CDCl}_{3}, 300 \mathrm{MHz}, 52{ }^{\circ} \mathrm{C}\right) \delta 6.23$ (br s, $\left.1 \mathrm{H}, \mathrm{NH}, 250\right), 5.97$ (br s, 1H, NH, 251), 5.82$5.63(\mathrm{~m}, 1 \mathrm{H}$, alkene $\mathrm{H}, \mathbf{2 5 1}), 4.99-4.88(\mathrm{~m}, 2 \mathrm{H}$, alkene $\mathrm{H}, \mathbf{2 5 1}), 4.15(\mathrm{~m}, 1 \mathrm{H}, \mathrm{CHN}, \mathbf{2 5 1}), 3.41-3.27\left(\mathrm{~m}, 2 \mathrm{H}, \mathrm{CH}_{2} \mathrm{~N}, 250\right), 2.16-$ 1.97 (m, 2H, $\mathrm{CH}_{2}$-alkene, 251), 2.16-1.13 (m, 8H, $\mathrm{CH}$ and $\left.\mathrm{CH}_{2}, \mathbf{2 5 0}\right), 1.82-1.13\left(\mathrm{~m}, 4 \mathrm{H}, \mathrm{CH}_{2}, \mathbf{2 5 1}\right), 1.44\left(\mathrm{~s}, 9 \mathrm{H}, \mathrm{OC}\left(\mathrm{CH}_{3}\right)_{3}, 250\right.$ and 251), $1.43\left(\mathrm{~s}, 9 \mathrm{H}, \mathrm{OC}\left(\mathrm{CH}_{3}\right)_{3}, 250\right.$ and 251$), 1.07\left(\mathrm{~d}, J=6.8 \mathrm{~Hz}, 3 \mathrm{H}, \mathrm{CHNCH}_{3}, \mathbf{2 5 1}\right), 0.95(\mathrm{~d}, J=6.5 \mathrm{~Hz}, 3 \mathrm{H}(40 \%), \mathrm{CHCH}$, 250 1.diastereoisomer), $0.82\left(\mathrm{~d}, J=6.9 \mathrm{~Hz}, 3 \mathrm{H}(60 \%), \mathrm{CHCH}_{3}, \mathbf{2 5 0} 2\right.$. diaseteroisomer); ${ }^{13} \mathrm{C} \mathrm{NMR}\left(\mathrm{CDCl}_{3}, 75 \mathrm{MHz}, 52{ }^{\circ} \mathrm{C}\right) \delta$ $155.8,155.4,154.8,138.5,114.4,114.2,80.7,53.1,50.6,45.9,41.2,38.2,35.5,34.7,33.6,31.7,30.7,28.6,28.3,28.3,28.2$, 27.4, 26.2, 25.8, 23.7, 22.5, 19.8, 18.0, 15.0, 14.0; IR v 3314 (w), 3076 (w), 2978 (m), $2932(\mathrm{~m}), 2871(\mathrm{w}), 1704(\mathrm{~s}), 1641(\mathrm{w})$, $1478(\mathrm{~m}), 1455(\mathrm{~m}), 1393(\mathrm{~s}), 1367(\mathrm{~s}), 1333(\mathrm{~m}), 1252(\mathrm{~m}), 1159(\mathrm{~s}), 1113(\mathrm{~m}), 1048(\mathrm{w}), 1015(\mathrm{w}), 910(\mathrm{w}), 857(\mathrm{w}), 759(\mathrm{w})$; MS(ESI) $329.2(\mathrm{M}+\mathrm{H}), 351.2(\mathrm{M}+\mathrm{Na})$; Anal. calcd for $\mathrm{C}_{17} \mathrm{H}_{32} \mathrm{~N}_{2} \mathrm{O}_{4}$ : C, 62.17; H, 9.82; N, 8.53. Found: C, 62.36; H, 9.99; N, 8.69 .

$N$-[5-( $N, N^{\prime}$-Di-tert-butoxycarbonyl-hydrazino)-1-methyl-hexyl]- $N$ '-(tert-butoxycarbonyl) hydrazinecarboxylic acid tertbutyl ester (252)

$\mathrm{R}_{\mathrm{f}}\left(\mathrm{AcOEt} / \mathrm{h}\right.$ exane 1:5) 0.15; Mp 156-158 ${ }^{\circ} \mathrm{C} ;{ }^{1} \mathrm{H} \mathrm{NMR}\left(\mathrm{CDCl}_{3}, 300 \mathrm{MHz}, 52{ }^{\circ} \mathrm{C}\right) \delta 6.20(\mathrm{br} \mathrm{s}, 2 \mathrm{H}, \mathrm{NH}), 4.19(\mathrm{~m}, 2 \mathrm{H}, \mathrm{CHN})$, $1.76-1.24\left(\mathrm{~m}, 6 \mathrm{H}, \mathrm{CH}_{2}\right), 1.49\left(\mathrm{~s}, 18 \mathrm{H}, \mathrm{OC}\left(\mathrm{CH}_{3}\right)_{3}\right), 1.45\left(\mathrm{~s}, 18 \mathrm{H}, \mathrm{OC}\left(\mathrm{CH}_{3}\right)_{3}\right), 1.08\left(\mathrm{~d}, \mathrm{~J}=6.8 \mathrm{~Hz}, 6 \mathrm{H}, \mathrm{CHNCH}_{3}\right) ;{ }^{13} \mathrm{C} \mathrm{NMR}$ $\left(\mathrm{CDCl}_{3}, 75 \mathrm{MHz}, 52{ }^{\circ} \mathrm{C}\right) \delta 155.9,155.0,80.7,52.7,34.3,28.4,23.6,18.4$; IR v $3315(\mathrm{~m}), 2978(\mathrm{~m}), 2932(\mathrm{~m}), 1732(\mathrm{~s}), 1707(\mathrm{~s})$, $1499(\mathrm{~m}), 1480(\mathrm{~m}), 1456(\mathrm{~m}), 1393$ (s), 1367 (s), $1343(\mathrm{~m}), 1296(\mathrm{~m}), 1274(\mathrm{~m}), 1245$ (s), 1158 (s), 1111 (m), 1096 (m), 1048 (w), 1017 (w), $962(\mathrm{w}), 920(\mathrm{w}), 859$ (w), 838 (w), 783 (w), 757 (w), 734 (m), 688 (w), 647 (w); MS(ESI) 583.4 (M+Na); Anal. calcd for $\mathrm{C}_{27} \mathrm{H}_{52} \mathrm{~N}_{4} \mathrm{O}_{8}$ : C, 57.83; H, 9.35; N, 9.99. Found: C, 57.67; H, 9.28; N, 10.00.

$N$-(4-Methyl-tetrahydro-furan-3-ylmethyl)- $N$ '-(tert-butoxycarbonyl)hydrazinecarboxylic acid tert-butyl ester (254)

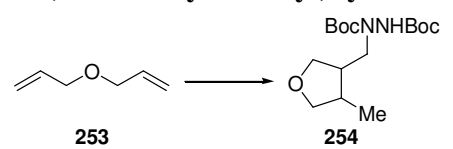

Following GP1 Hydrohydrazination product 254 (mixture of diastereoisomers trans/cis 1:1.6, $113 \mathrm{mg}, 0.342 \mathrm{mmol}, 68 \%)$ was obtained as a colorless solid with diallyl ether (253) $(61 \mu \mathrm{L}, 0.50$ mmol, $1.0 \mathrm{eq}$ ) in $15 \mathrm{~h}$ with $5 \mathrm{~mol} \%$ catalyst $\mathbf{2 0}$ after purification by column chromatography (AcOEt/hexane 1:10-1:2). Analytically pure samples of the trans and the cis isomers could be obtained after two more column chromatographies (AcOEt/hexane 1:15-1:5). Following GP3 Hydrohydrazination product 254 (mixture of diastereoisomers trans/cis 1:2.5, $145 \mathrm{mg}, 0.439$ mmol, 88\%) was obtained as a colorless solid with diallyl ether (253) $(61 \mu \mathrm{L}, 0.50 \mathrm{mmol}, 1.0 \mathrm{eq})$ in $24 \mathrm{~h}$ with $2 \mathrm{~mol} \%$ catalyst $\mathbf{2 3}$ after purification by column chromatography (AcOEt/hexane $1: 10-1: 2)$. Analytically pure samples of the trans and the cis isomers could be obtained after two more column chromatographies (AcOEt/hexane 1:15-1:5).

\section{trans-254}

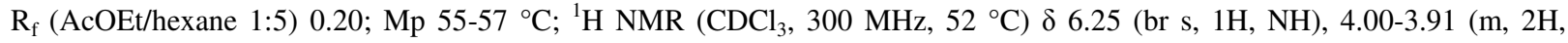
$\left.\mathrm{CH}_{2} \mathrm{O}\right)$, 3.64-3.50 (m, 2H, $\mathrm{CH}_{2} \mathrm{O}$ and/or $\left.\mathrm{CH}_{2} \mathrm{~N}\right), 3.42-3.28\left(\mathrm{~m}, 2 \mathrm{H}, \mathrm{CH}_{2} \mathrm{O}\right.$ and/or $\left.\mathrm{CH}_{2} \mathrm{~N}\right), 2.15-2.03\left(\mathrm{~m}, 1 \mathrm{H}, \mathrm{CHCH} \mathrm{H}_{2} \mathrm{~N}\right), 2.00-1.91$ $\left(\mathrm{m}, 1 \mathrm{H}, \mathrm{CHCH}_{3}\right), 1.48\left(\mathrm{~s}, 9 \mathrm{H}, \mathrm{OC}\left(\mathrm{CH}_{3}\right)_{3}\right), 1.47\left(\mathrm{~s}, 9 \mathrm{H}, \mathrm{OC}\left(\mathrm{CH}_{3}\right)_{3}\right), 1.05\left(\mathrm{~d}, J=6.5 \mathrm{~Hz}, 3 \mathrm{H}, \mathrm{CHCH}_{3}\right) ;{ }^{1} \mathrm{H} \mathrm{NMR}\left(\mathrm{CDCl}_{3}, 500 \mathrm{MHz}\right.$, $23{ }^{\circ} \mathrm{C}$ ): NOE 


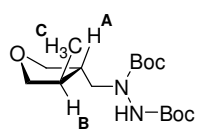

pulse on A (2.09): NOE on $\mathbf{B}$ not observed, NOE on $\mathbf{C}$ strong; pulse on $\mathbf{B}$ (1.96): NOE on $\mathbf{A}$ not observed, NOE on $\mathbf{C}$ strong; pulse on $\mathbf{C}$ (1.05): NOE on A strong, NOE on $\mathbf{B}$ strong; ${ }^{13} \mathrm{C} \mathrm{NMR}\left(\mathrm{CDCl}_{3}, 75 \mathrm{MHz}, 52{ }^{\circ} \mathrm{C}\right) \delta 155.4,81.4,74.9,72.0,52.4,46.1$, 37.6, 28.2, 17.0; IR v $3305(\mathrm{~m}), 2977(\mathrm{~s}), 2932(\mathrm{~m}), 2873(\mathrm{~m}), 1713(\mathrm{~s}), 1479(\mathrm{~m}), 1455$ (m), $1393(\mathrm{~s}), 1367$ (s), $1254(\mathrm{~s}), 1149$ (s), $1048(\mathrm{~m}), 1018(\mathrm{~m}), 922(\mathrm{~m}), 857(\mathrm{w}), 781(\mathrm{w}), 758(\mathrm{~m}), 734(\mathrm{~m}), 698(\mathrm{w}), 647(\mathrm{w})$; MS(ESI) $331.4(\mathrm{M}+\mathrm{H}), 353.4(\mathrm{M}+\mathrm{Na})$; Anal. calcd for $\mathrm{C}_{16} \mathrm{H}_{30} \mathrm{~N}_{2} \mathrm{O}_{5}$ : C, 58.16; H, 9.15; N, 8.48. Found: C, 57.74; H, 9.39; N, 8.67.

\section{cis-254}

$\mathrm{R}_{\mathrm{f}}$ (AcOEt/hexane 1:5) 0.20; $\mathrm{Mp} \mathrm{88-89}{ }^{\circ} \mathrm{C} ;{ }^{1} \mathrm{H} \mathrm{NMR}\left(\mathrm{CDCl}_{3}, 300 \mathrm{MHz}, 52{ }^{\circ} \mathrm{C}\right) \delta 6.30$ (br s, $\left.1 \mathrm{H}, \mathrm{NH}\right), 3.92-3.87(\mathrm{~m}, 2 \mathrm{H}$, $\left.\mathrm{CH}_{2} \mathrm{O}\right), 3.63-3.41\left(\mathrm{~m}, 4 \mathrm{H}, \mathrm{CH}_{2} \mathrm{O}\right.$ and $\left.\mathrm{CH}_{2} \mathrm{~N}\right), 2.58-2.46\left(\mathrm{~m}, 1 \mathrm{H}, \mathrm{CHCH}_{2} \mathrm{~N}\right), 2.41-2.28\left(\mathrm{~m}, 1 \mathrm{H}, \mathrm{CHCH}_{3}\right), 1.46\left(\mathrm{~s}, 9 \mathrm{H}, \mathrm{OC}\left(\mathrm{CH}_{3}\right)_{3}\right)$, $1.45\left(\mathrm{~s}, 9 \mathrm{H}, \mathrm{OC}\left(\mathrm{CH}_{3}\right)_{3}\right), 0.96\left(\mathrm{~d}, J=7.2 \mathrm{~Hz}, 3 \mathrm{H}, \mathrm{CHCH}_{3}\right) ;{ }^{1} \mathrm{H} \mathrm{NMR}\left(\mathrm{CDCl}_{3}, 500 \mathrm{MHz}, 23{ }^{\circ} \mathrm{C}\right): \mathrm{NOE}$

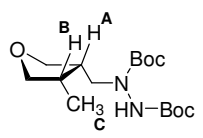

pulse on A (2.52): NOE on $\mathbf{B}$ strong, NOE on $\mathbf{C}$ weak; pulse on $\mathbf{B}$ (2.34): NOE on $\mathbf{A}$ strong, NOE on $\mathbf{C}$ strong; pulse on $\mathbf{C}$ (0.96): NOE on $\mathbf{A}$ weak, NOE on $\mathbf{B}$ strong; ${ }^{13} \mathrm{C}$ NMR $\left(\mathrm{CDCl}_{3}, 75 \mathrm{MHz}, 52{ }^{\circ} \mathrm{C}\right) \delta 155.2,81.3,75.1,70.9,48.6,40.9,35.5,28.3$, 12.9; IR v 3309 (m), 2976 (s), 2932 (m), 2877 (m), 1711 (s), 1479 (m), 1455 (m), 1393 (s), 1367 (s), 1320 (m), 1276 (s), 1254 (s), 1148 (s), 1048 (m), 1019 (w), 913 (w), 856 (w), $781(w), 758$ (w), 734 (w), 647 (w); MS(ESI) $331.4(\mathrm{M}+\mathrm{H}), 353.4$ (M+Na); Anal. calcd for $\mathrm{C}_{16} \mathrm{H}_{30} \mathrm{~N}_{2} \mathrm{O}_{5}$ : C, 58.16; H, 9.15; N, 8.48. Found: C, 58.04; H, 9.17; N, 8.37.

3-( $N, N^{\prime}$-Di-tert-butoxycarbonyl-hydrazinomethyl)-4-methyl-cyclopentane-1,1-dicarboxylic acid diethyl ester (256)

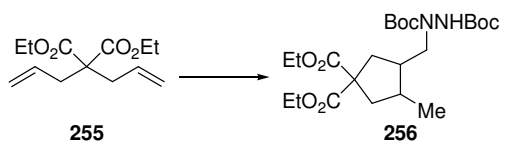

Following GP1 Hydrohydrazination product 256 (mixture of diastereoisomers 7:1, $143 \mathrm{mg}, 0.303 \mathrm{mmol}, 62 \%$ ) was obtained as a colorless solid with diallylmalonate $255(118 \mathrm{mg}, 0.491 \mathrm{mmol}, 1.00 \mathrm{eq})$ in $15 \mathrm{~h}$ with 5 mol\% catalyst 20 after purification by column chromatography (AcOEt/hexane 1:10-1:5). Following GP3 Hydrohydrazination product 256 (mixture of diastereoisomers 9:1, $221 \mathrm{mg}, 0.468 \mathrm{mmol}, 93 \%)$ was obtained as a colorless solid with diallylmalonate 255 (121 mg, 0.504 mmol, $1.00 \mathrm{eq}$ ) in $4 \mathrm{~h}$ with $2 \mathrm{~mol} \%$ catalyst 23 after purification by column chromatography (AcOEt/hexane 1:10-1:5). $\mathrm{R}_{\mathrm{f}}$ (AcOEt/hexane 1:2) 0.45; Mp 82-85 ${ }^{\circ} \mathrm{C} ;{ }^{1} \mathrm{H} \mathrm{NMR}\left(\mathrm{CDCl}_{3}, 300 \mathrm{MHz}, 52{ }^{\circ} \mathrm{C}\right) \delta 6.24$ (br s, $\left.1 \mathrm{H}, \mathrm{NH}\right), 4.19-4.05\left(\mathrm{~m}, 2 \mathrm{H}, \mathrm{CH}_{2} \mathrm{O}\right)$, 3.51-3.31 (m, $\left.2 \mathrm{H}, \mathrm{CH}_{2} \mathrm{~N}\right), 2.48-1.64\left(\mathrm{~m}, 6 \mathrm{H}, \mathrm{CH}\right.$ and $\left.\mathrm{CH}_{2}\right), 1.44\left(\mathrm{~s}, 9 \mathrm{H}, \mathrm{OC}\left(\mathrm{CH}_{3}\right)_{3}\right), 1.43\left(\mathrm{~s}, 9 \mathrm{H}, \mathrm{OC}\left(\mathrm{CH}_{3}\right)_{3}\right), 1.21(\mathrm{t}, J=7.2 \mathrm{~Hz}$, $\left.\mathrm{CH}_{2} \mathrm{CH}_{3}\right), 0.98\left(\mathrm{~d}, \mathrm{~J}=5.9 \mathrm{~Hz}, 3 \mathrm{H}, \mathrm{CHCH}_{3}\right.$, minor diastereoisomer), 0.87 (d, $\mathrm{J}=6.8 \mathrm{~Hz}, 3 \mathrm{H}, \mathrm{CHCH}$, major diastereoisomer); ${ }^{13} \mathrm{C}$ $\mathrm{NMR}\left(\mathrm{CDCl}_{3}, 75 \mathrm{MHz}, 52{ }^{\circ} \mathrm{C}\right) \delta 172.5,172.3,155.2,81.1,61.3,59.0,50.2,41.7,40.7,36.9,35.1,28.3,14.9,14.0,13.9$; IR v $3337(\mathrm{w}), 2978(\mathrm{~m}), 2934(\mathrm{~m}), 1731$ (s), $1478(\mathrm{~m}), 1456(\mathrm{~m}), 1393(\mathrm{~m}), 1367(\mathrm{~s}), 1254(\mathrm{~s}), 1152(\mathrm{~s}), 1096(\mathrm{~m}), 1054(\mathrm{~m}), 1018$ (m), $918(w), 859(w), 782(w), 758(w), 734(w) ; M S(E S I) 495.4(\mathrm{M}+\mathrm{Na})$; Anal. calcd for $\mathrm{C}_{23} \mathrm{H}_{40} \mathrm{~N}_{2} \mathrm{O}_{8}$ : C, 58.46; H, 8.53; N, 5.93. Found: C, 58.42; H, 8.43; N, 5.97.

\section{Equation Used for the Estimation of the Reaction Rate ${ }^{57}$}

$$
\frac{k_{1}}{k_{2}} \bullet\left\{\ln \left([X Y]_{i}+\frac{k_{1}}{k_{2}}\right)-\ln \left([X Y]_{f}+\frac{k_{1}}{k_{2}}\right)\right\}=[S u b]_{i} \bullet \frac{\left[R^{\prime} X\right] /[R X]}{1+\left[R^{\prime} X\right] /[R X]}
$$

In this equation, $\mathrm{k}_{1}$ is the rate of the known unimolecular radical reaction, $\mathrm{k}_{2}$ the unknown rate for the bimolecular amination reaction; $[\mathrm{XY}]_{\mathrm{i}}$ is the initial concentration $(0.3 \mathrm{M})$ and $[\mathrm{XY}]_{\mathrm{f}}$ is the final concentration $(0 \mathrm{M})$ of the radical trap (azodicarboxylate 2); $[\mathrm{Sub}]_{\mathrm{i}}$ is the initial concentration of alkene $(0.2 \mathrm{M})$; $[\mathrm{RX}]$ and $\left[\mathrm{R}^{\prime} \mathrm{X}\right]$ are the yields of unrearranged and rearranged products respectively. The solution of this equation was approximated numerically.

\section{Specific Values}

For $\boldsymbol{\alpha}$-phenylvinylcyclopropane (247): Co-catalyst 20: $[R X]=0.12 \mathrm{M},\left[\mathrm{R}^{\prime} \mathrm{X}\right]=0.04 \mathrm{M}, \mathrm{k}_{1}=4 \cdot 10^{5} \mathrm{~s}^{-1}, \mathrm{k}_{2}=1.5 \bullet 10^{8} \mathrm{~s}^{-1} \mathrm{~mol}^{-1}$; Mn-catalyst 23: $[R X]=0.10 \mathrm{M},\left[\mathrm{R}^{\prime} \mathrm{X}\right]=0.05 \mathrm{M}, \mathrm{k}_{1}=4 \cdot 10^{5} \mathrm{~s}^{-1}, \mathrm{k}_{2}=2.5 \cdot 10^{8} \mathrm{~s}^{-1} \mathrm{~mol}^{-1}$. For 1,6-heptadiene (249): Co-catalyst 20: $[R X]=0.13 \mathrm{M},\left[\mathrm{R}^{\prime} \mathrm{X}\right]=0.016 \mathrm{M}, \mathrm{k}_{1}=1 \cdot 10^{5} \mathrm{~s}^{-1}, \mathrm{k}_{2}=1.5-2.0 \cdot 10^{8} \mathrm{~s}^{-1} \mathrm{~mol}^{-1} ;$ Mn-catalyst 23: $[\mathrm{RX}]=0.13 \mathrm{M},[\mathrm{R} \mathrm{X}]=0.013 \mathrm{M}, \mathrm{k}_{1}=$ $1 \cdot 10^{5} \mathrm{~s}^{-1}, \mathrm{k}_{2}=2-2.5 \cdot 10^{8} \mathrm{~s}^{-1} \mathrm{~mol}^{-1}$.

\subsection{Kinetic Measurements \\ 4.3.1. Methods and Formula}

NMR-Method for the Monitoring of the Hydrohydrazination of 4-phenylbutene (1)

A mixture of Co catalyst 20 and azodicarboxylate 2 were dissolved in $\mathrm{CD}_{3} \mathrm{OD}(0.75 \mathrm{~mL})$ at $23{ }^{\circ} \mathrm{C}$ under argon in a NMR tube. 4-Phenylbutene (1) was added, followed by the silane (time set to zero at this point). The reaction was monitored by ${ }^{1} H$-NMR at $300 \mathrm{MHz}$. Systematic variations of one of the reagent starting from the standard conditions (4-phenyl butene (1): $19 \mu \mathrm{L}, 0.13$ 
mmol, $0.17 \mathrm{M}, 1.0$ equiv; catalyst 20: $2 \mathrm{mg}, 0.005 \mathrm{mmol}, 0.007 \mathrm{M}, 0.04$ equiv; azodicarboxylate 2: $43 \mathrm{mg}, 0.20 \mathrm{mmol}, 0.27 \mathrm{M}$, 1.5 equiv; phenylsilane: $16 \mu \mathrm{L}, 0.13 \mathrm{mmol}, 0.17 \mathrm{M}, 1.0$ equiv; $25{ }^{\circ} \mathrm{C}$; $0.75 \mathrm{~mL} \mathrm{CD}_{3} \mathrm{OD}$ ) were examined. The ratio of hydrohydrazination product 3 to alkene 1 was calculated from the ratio of the integrals between 1.14 and $1.00 \mathrm{ppm}\left(\mathrm{CH}_{3}\right.$ group of 3) and between 2.40 and $2.23 \mathrm{ppm}$ (alkene- $\mathrm{CH}_{2}$ group of $\mathbf{1}$ ). The concentration of alkene $\mathbf{1}$ was then calculated from this ratio and the initial concentration. In this way, the large absolute error of the NMR method was minimized. The relative values obtained present the advantage of being easily compared and requires less experimental work than absolute values based on internal standards.

\section{GC-Method for Monitoring of the Hydrohydrazination of 4-phenylbutene (1)}

The hydrohydrazination of 4-phenylbutene (1) with TMDSO following GP11 was used as reference for these studies. Each reagent was then varied systematically (see Appendix A). Gas chromatographic measurements: An aliquot of $0.10 \mathrm{~mL}$ of the reaction mixture was diluted with $0.90 \mathrm{~mL}$ hexane and filtered through a short silica gel plug $(2.5 \mathrm{~cm})$ to remove the catalyst. 2 $\mu \mathrm{L}$ of the obtained solution were injected in the gas chromatographer (HP 6890 Series GC System) and the following temperature program was followed: - $5 \mathrm{~min}$ at $60{ }^{\circ} \mathrm{C}$ - to $90{ }^{\circ} \mathrm{C}$ with a temperature gradient of $5{ }^{\circ} \mathrm{C} / \mathrm{min}$ - to $140{ }^{\circ} \mathrm{C}$ with a temperature gradient of $12{ }^{\circ} \mathrm{C} / \mathrm{min}$ - to $180{ }^{\circ} \mathrm{C}$ with a temperature gradient of $8{ }^{\circ} \mathrm{C} / \mathrm{min}$ - $10 \mathrm{~min}$ at $180{ }^{\circ} \mathrm{C}$ Using this procedure, 4phenylbutene (1) eluted after $12.65 \mathrm{~min}$, 4-phenylbutane (44) after $12.48 \mathrm{~min}$ and (3-azido-butyl)-benzene (33) after $18.00 \mathrm{~min}$. The ratio of hydroazidation product $\mathbf{3 3}$ to alkene $\mathbf{1}$ was calculated from the ratio of the integrals of the signals after 12.65 min and $18.00 \mathrm{~min}$. The concentration of alkene 1 was then calculated from this ratio and the initial concentration. In the rare cases where the formation of 4-phenylbutane (44) was significant, the concentration was further corrected.

\section{Statistical Methods and Formula ${ }^{58}$}

\section{Reaction Rate}

The initial rates $r$ of the reactions were determined using standard linear regression programs (Excel) applied on the linear region of the concentration curves. The standard deviation and the confidence interval of the data were calculated using following formula:

$$
\text { Standard deviation of the rate: } s_{r}^{2}=\frac{n}{n \cdot \sum_{i=1}^{n} t_{i}^{2}-\left(\sum_{i=1}^{n} t_{i}\right)^{2}} \cdot \sum_{i=1}^{n}\left(c_{i}-I-r \cdot t_{i}\right)^{2} \cdot \frac{1}{n-2} \quad \text { Confidence interval (95\%) of the rate: ; } \quad v_{r}=t_{s} \cdot \frac{s_{r}}{\sqrt{n}}
$$

Whereas $\mathrm{n}$ is the amount of data points measured, $\mathrm{t}$ is the time of measurement after the addition of the silane, $\mathrm{c}$ is the measured concentration of 4-phenylbutene $(\mathbf{1}), r$ is the calculated reaction rate, $I$ is the calculate intercept of the curve, $t_{s}$ is the student- $t$ factor corresponding to $95 \%$ probability and a degree of freedom of n-2.

\section{Van't Hoff Equation}

Van't Hoff Equation: $y=\log r=O \cdot \log c+I=a \cdot \log c+b$;

Standard deviation, confidence interval for y values: $s_{y i}=\frac{s_{r i}}{\operatorname{Ln}(10) \cdot r_{i}}, \quad v_{y i}=\frac{v_{r i}}{\operatorname{Ln}(10) \cdot r_{i}}$

Standard deviation of the reaction order:

$$
s_{O}=s_{a}=\frac{\sum_{i=1}^{m} \frac{1}{s_{y i}^{2}}}{\sum_{i=1}^{m} \frac{1}{s_{y i}^{2}} \cdot \sum_{i=1}^{m} \frac{x_{i}^{2}}{s_{y i}^{2}}-\left(\sum_{i=1}^{m} \frac{x_{i}}{s_{y i}^{2}}\right)^{2}}, x_{i}=\log c_{i}
$$

Confidence interval (95\%) of the reaction order: ; $v_{O}=v_{a}=t_{s} \cdot \frac{s_{a}}{\sqrt{m}}$

Correlation factor R: $\frac{\sum_{i=1}^{m} \frac{1}{s_{y i}^{2}} \cdot \sum_{i=1}^{m} \frac{x_{i} y_{i}}{s_{y i}^{2}}-\sum_{i=1}^{m} \frac{x_{i}}{s_{y i}^{2}} \cdot \sum_{i=1}^{m} \frac{y_{i}}{s_{y i}^{2}}}{\sqrt{\sum_{i=1}^{m} \frac{1}{s_{y i}^{2}} \cdot \sum_{i=1}^{m} \frac{x_{i}^{2}}{s_{y i}^{2}}-\left(\sum_{i=1}^{m} \frac{x_{i}}{s_{y i}^{2}}\right)^{2}} \cdot \sqrt{\sum_{i=1}^{m} \frac{1}{s_{y i}^{2}} \cdot \sum_{i=1}^{m} \frac{y_{i}^{2}}{s_{y i}^{2}}-\left(\sum_{i=1}^{m} \frac{y_{i}}{s_{y i}^{2}}\right)^{2}}}$

Whereas $\mathrm{r}$ is the calculated reaction rate with standard deviation $\mathrm{s}_{\mathrm{r}}$ and confidence interval $\mathrm{v}_{\mathrm{r}}, \mathrm{O}$ is the reaction order with standard deviation $\mathrm{s}_{\mathrm{O}}$ and confidence interval $\mathrm{v}_{\mathrm{O}}$, $\mathrm{c}$ is the concentration of the examined reagent, $\mathrm{m}$ is the amount of data points measured, $\mathrm{R}$ is the correlation factor, $\mathrm{t}_{\mathrm{s}}$ is the student- $\mathrm{t}$ factor corresponding to $95 \%$ probability and a degree of freedom of $\mathrm{n}-2$.

\section{Arrhenius Equation}

Arrhenius equation: $y=\log r=-\frac{E_{a}}{R} \cdot \frac{1}{T}+\log A+\log K=a \cdot \frac{1}{T}+b$ Standard deviation, confidence interval for y values: $s_{y i}=\frac{s_{r i}}{r_{i}}, \quad v_{y i}=\frac{v_{r i}}{r_{i}}$

Energy of activation: $E_{a}=-R \cdot a$ Standard deviation of the energy of activation: $s_{E a}=R \cdot s_{a}$ Confidence interval (95\%) of the energy of activation: $v_{E a}=R \cdot v_{a}$ Whereas $\mathrm{r}$ is the calculated reaction rate with standard deviation $\mathrm{s}_{\mathrm{r}}$ and confidence interval $\mathrm{v}_{\mathrm{r}}, \mathrm{R}=8.314$ $\mathrm{J} \cdot \mathrm{mol}^{-1} \mathrm{~K}^{-1}$, A is the Arrhenius parameter, $\mathrm{K}$ is a concentration-dependent correction factor, $\mathrm{s}_{\mathrm{a}}$ and $\mathrm{v}_{\mathrm{a}}$ and the correlation factor $\mathrm{R}$ are obtained with the same formula as for the van't Hoff equation.

Kinetic Isotope Effect

Kinetic isotope effect: $k_{i s o}=\frac{r_{H}}{r_{D}}$ Standard deviation of the kinetic isotope effect: $\quad s_{k_{i s o}}=\sqrt{\left(\frac{s_{r_{H}}}{r_{D}}\right)^{2}+\left(\frac{r_{H} \cdot s_{r_{D}}}{r_{D}^{2}}\right)^{2}}$

Confidence interval (95\%) of the kinetic isotope effect: $\quad v_{k_{\text {too }}}=\sqrt{\left(\frac{v_{r_{H}}}{r_{D}}\right)^{2}+\left(\frac{r_{H} \cdot v_{r_{D}}}{r_{D}^{2}}\right)^{2}}$ 
Whereas $\mathrm{r}_{\mathrm{H}}$ is the calculated reaction rate with $\mathrm{PhSiH}_{3}$ with standard deviation $\mathrm{s}_{\mathrm{rH}}$ and confidence interval $\mathrm{v}_{\mathrm{rH}}, \mathrm{r}_{\mathrm{D}}$ is the calculated reaction rate with $\mathrm{PhSiD}_{3}$ with standard deviation $\mathrm{S}_{\mathrm{rD}}$ and confidence interval $\mathrm{V}_{\mathrm{rD}}$.

All the calculated date in the form value(standard deviation) \pm confidence interval $(95 \%)$ and the measured data are given in the Appendix A.

\subsubsection{Graphical Description of the Results}

Figure 1: Hydrohydrazination of 4-phenylbutene (1): dependence of the reaction rate on the initial concentration of $\mathbf{1}$. The lines represent the calculated initial rates. ${ }^{59}$

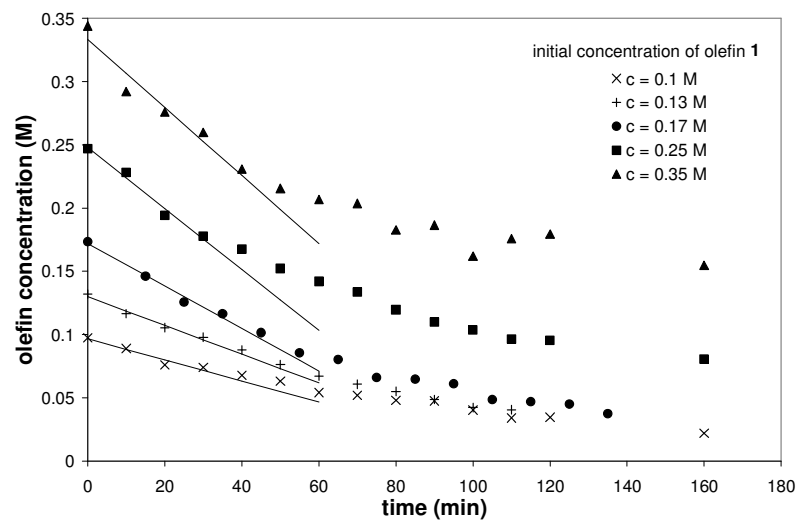

Figure 2: $\quad$ Hydrohydrazination of 4-phenylbutene (1): dependence of the reaction rate on the initial concentration of azodicarboxylate $\mathbf{2}$. The lines represent the calculated initial rates.

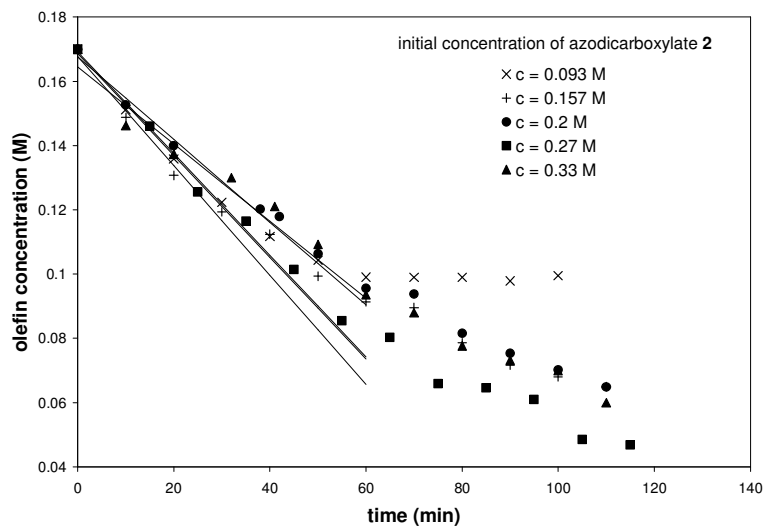

Figure 3: Hydrohydrazination of 4-phenylbutene (1): dependence of the reaction rate on the initial concentration of phenylsilane. The lines represent the calculated initial rates.

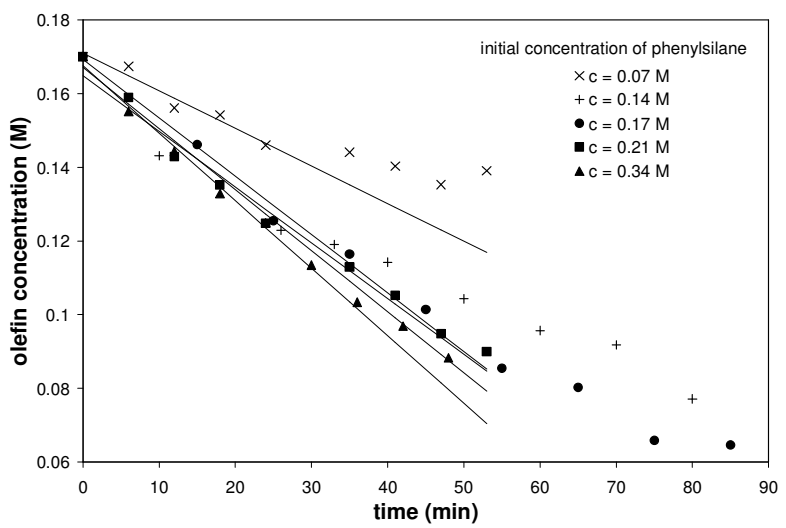


Figure 4: Hydrohydrazination of 4-phenylbutene (1): dependence of the reaction rate on the initial concentration of catalyst (20). The lines represent the calculated initial rates.

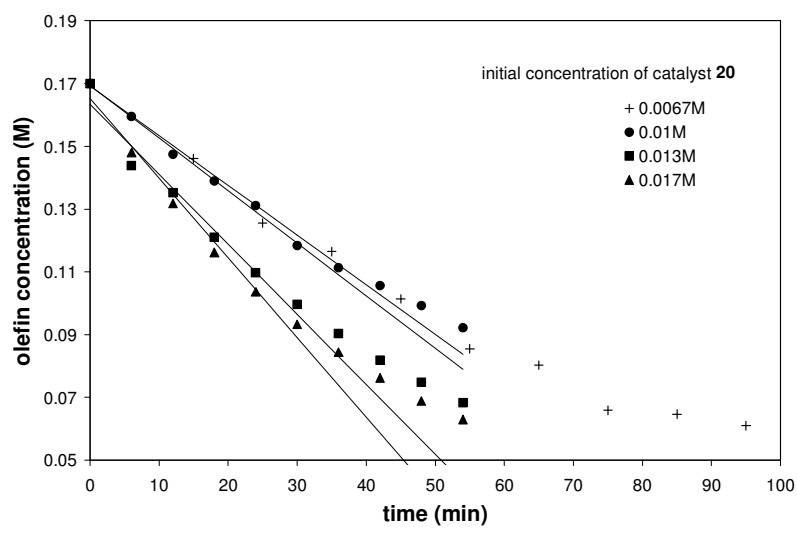

Figure 5: van't Hoff plots for the determination of the order of the reactants in the hydrohydrazination of 4-phenylbutene (1).
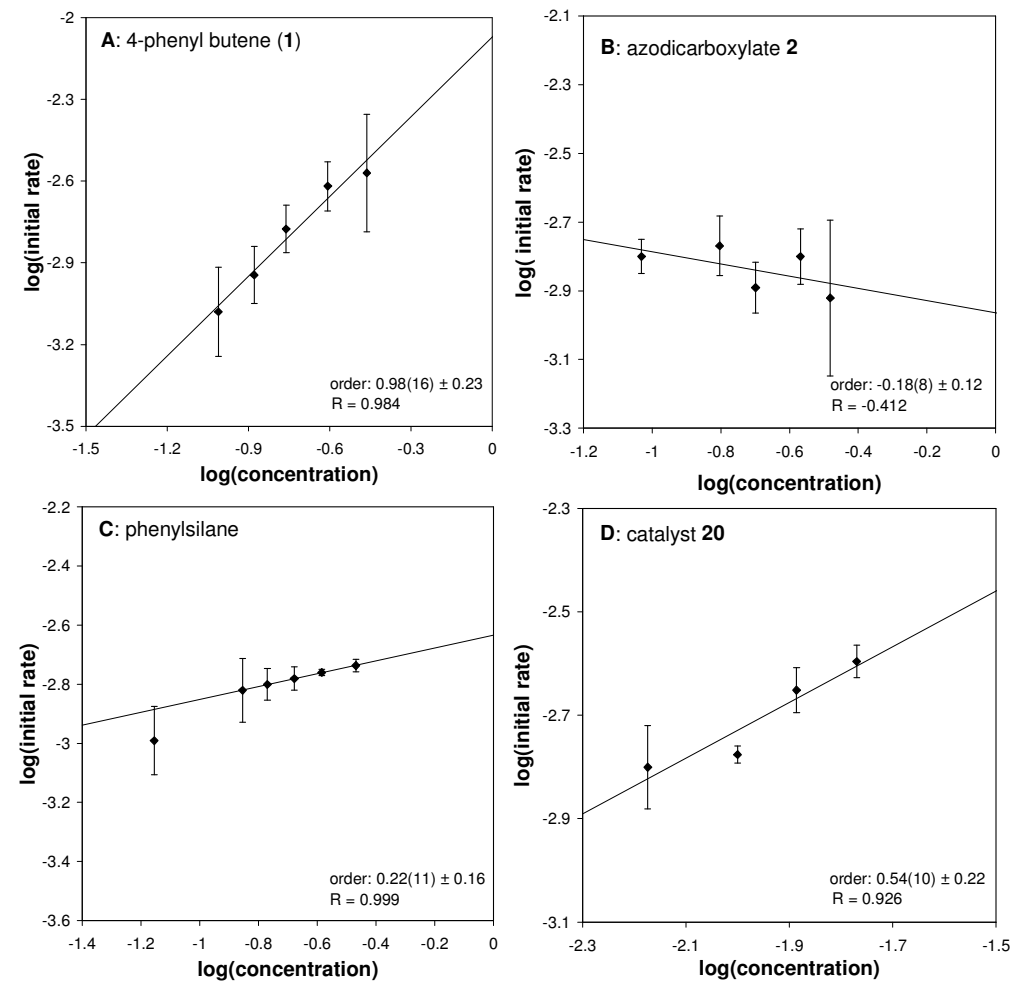
Figure 6: Dependence of the reaction rate on the temperature and Arrhenius plot for the hydrohydrazination of 4-phenylbutene (1).
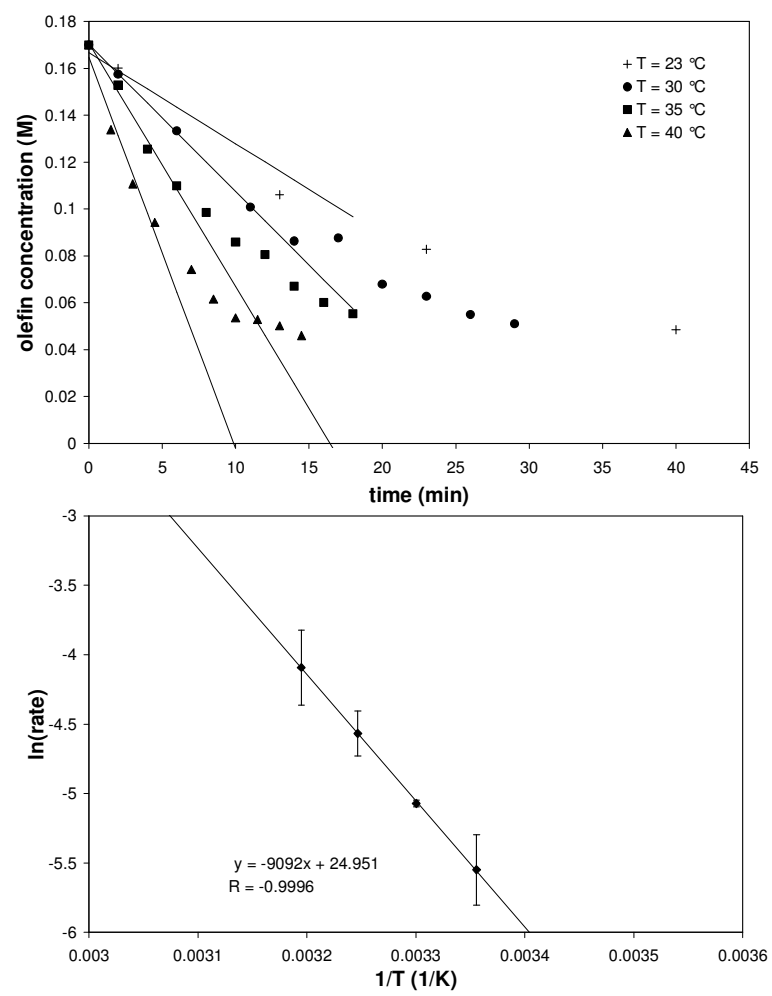

Figure 7: Kinetic isotope effect in the hydrohydrazination of 4-phenylbutene (1).

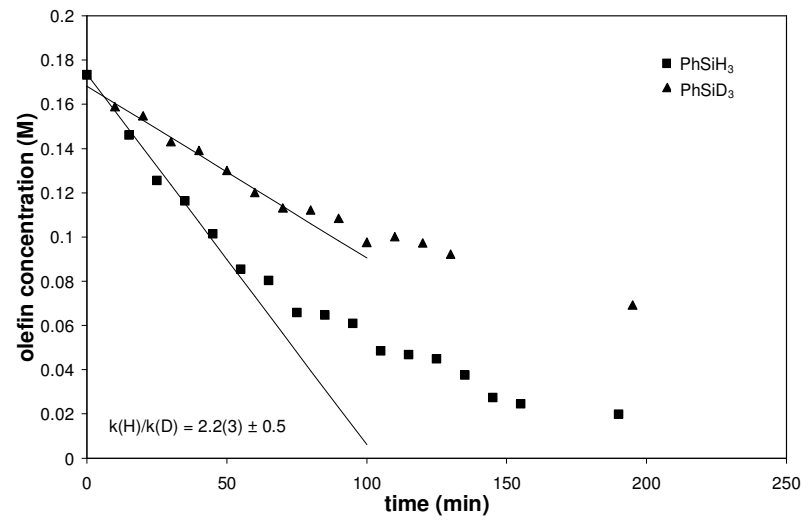


Figure 8: Hydroazidation of 4-phenylbutene (1): dependence of the reaction rate on the initial concentration of $\mathbf{1}$. The lines represent the calculated initial rates.

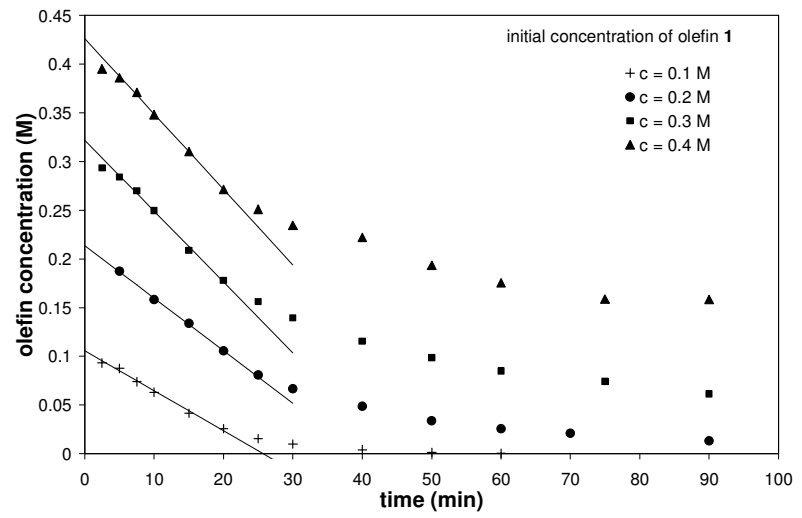

Figure 9 Hydroazidation of 4-phenylbutene (1): dependence of the reaction rate on the initial concentration of tosyl azide (203). The lines represent the calculated initial rates.

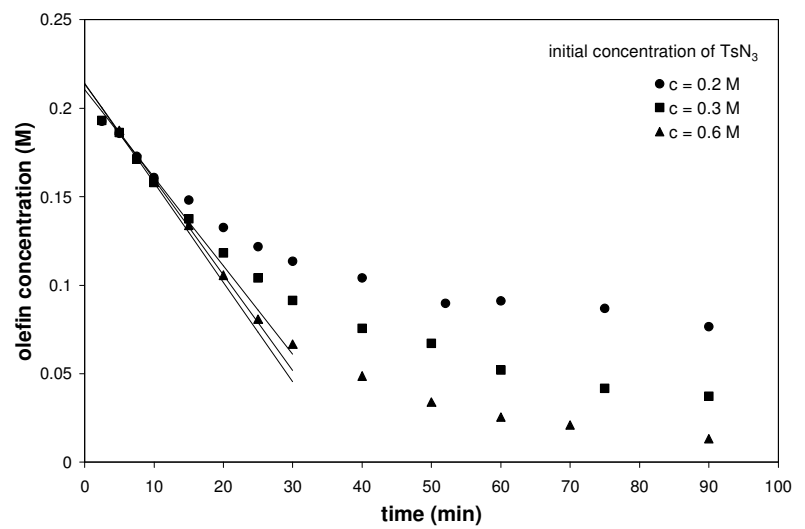

Figure 10: Hydroazidation of 4-phenylbutene (1): dependence of the reaction rate on the initial concentration of TMDSO. The lines represent the calculated initial rates.

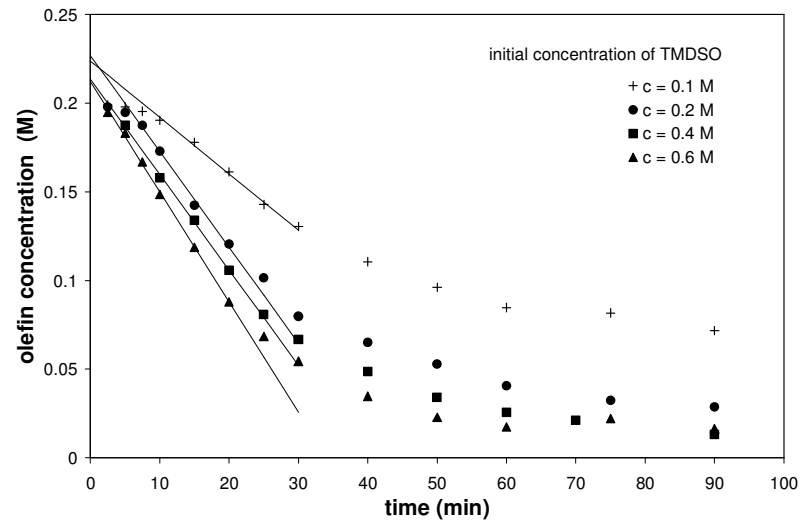


Figure 11: Hydroazidation of 4-phenylbutene (1): dependence of the reaction rate on the initial concentration of $\mathrm{Co}\left(\mathrm{BF}_{4}\right) \cdot 6 \mathrm{H}_{2} \mathrm{O}$ and ligand 43 in ratio 1:1. The lines represent the calculated initial rates.

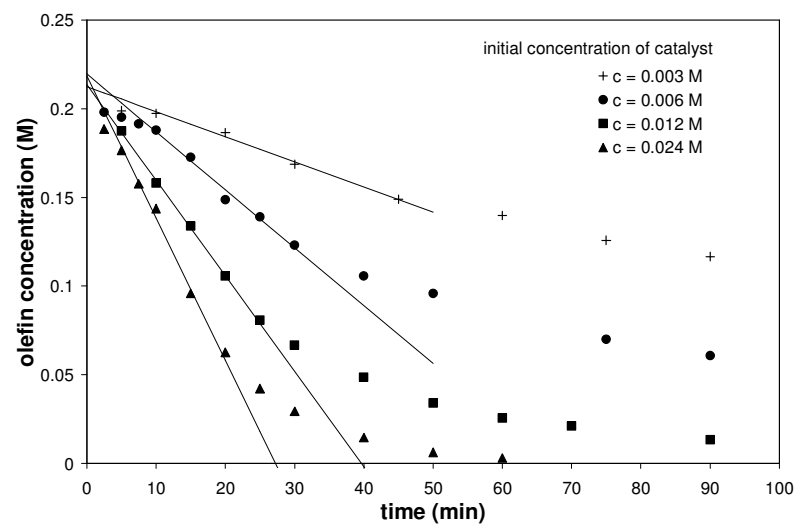

Figure 12: Hydroazidation of 4-phenylbutene (1): dependence of the reaction rate on the initial concentration of tert-butyl hydroperoxide. The lines represent the calculated initial rates.

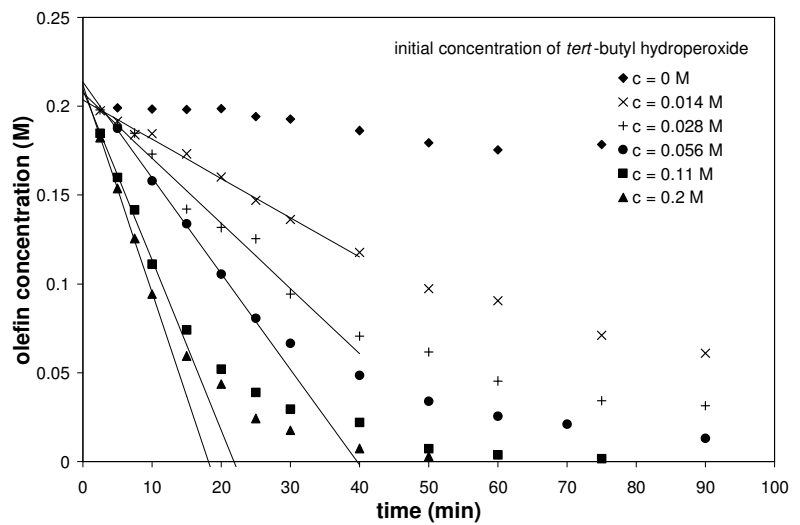


Figure 13: van't Hoff plots for the determination of the order of the reactants in the hydroazidation of 4phenylbutene (1).
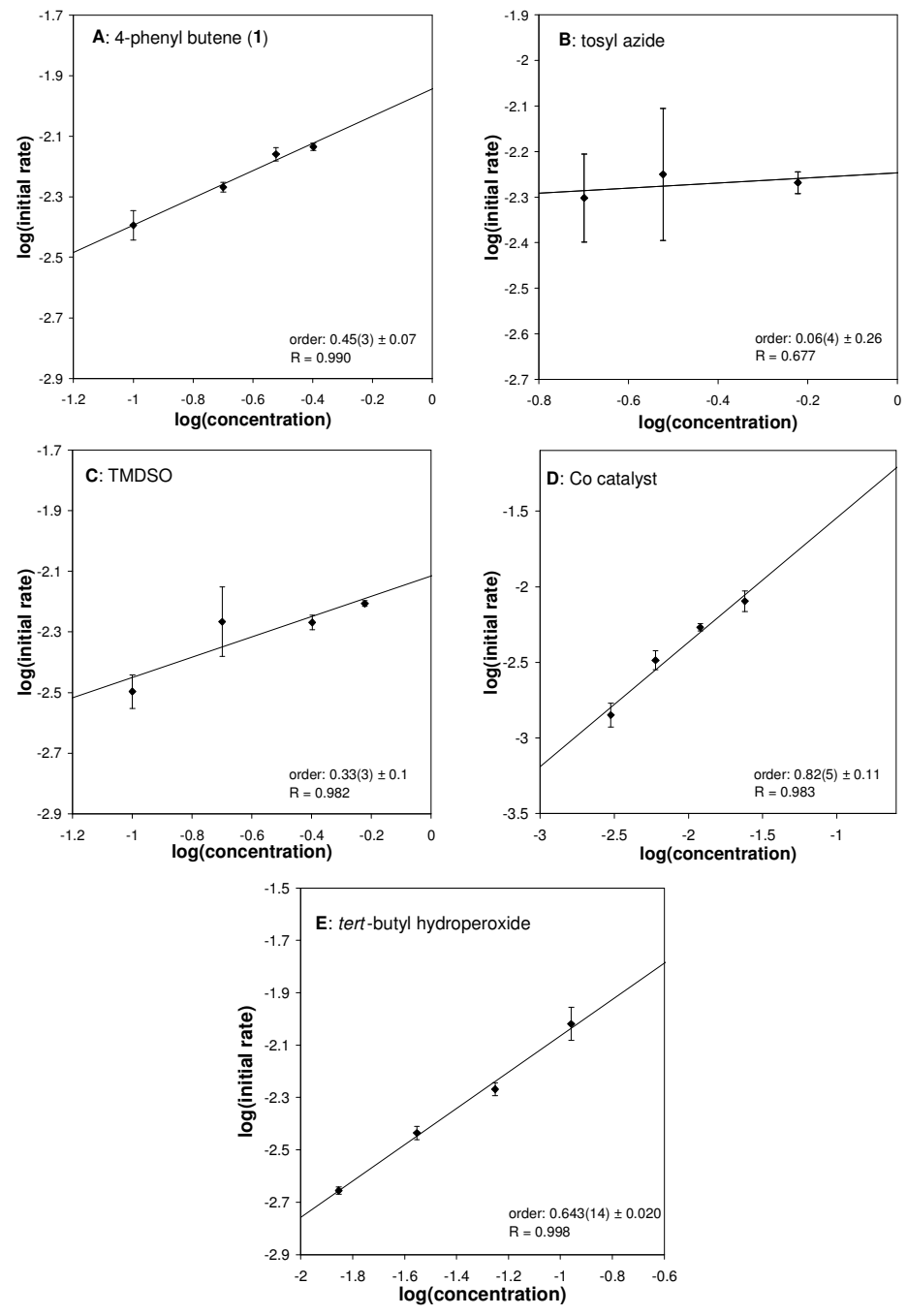
Figure 14: Dependence of the reaction rate on the temperature and Arrhenius plot for the hydroazidation of 4-phenylbutene (1).
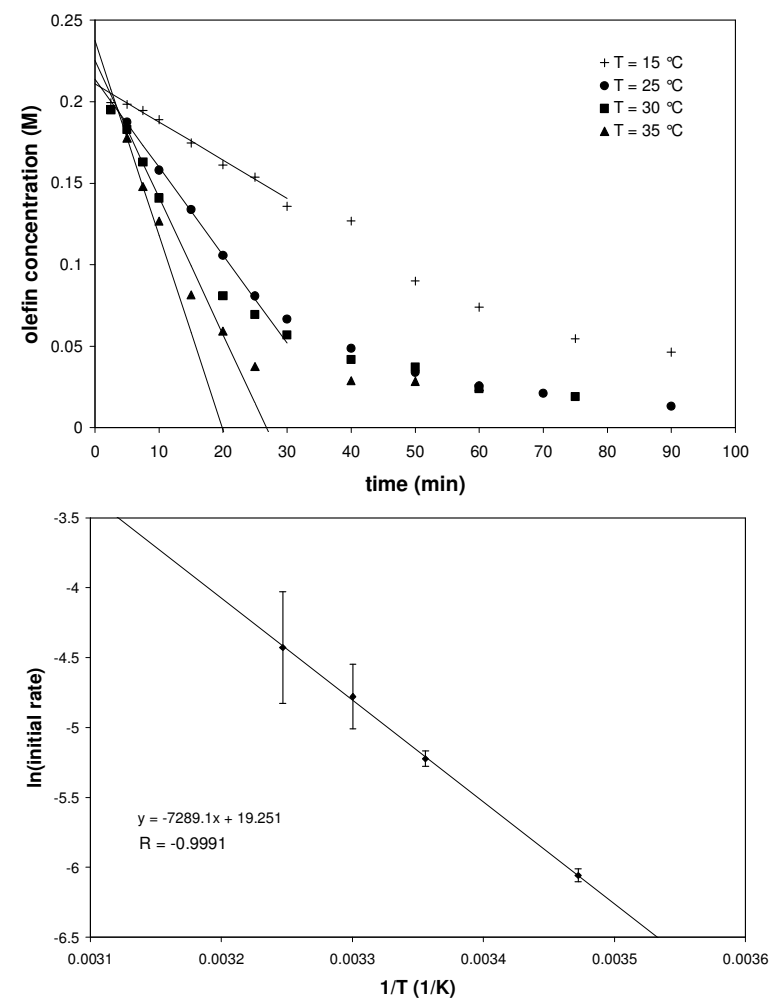

Figure 15: Kinetic isotope effect in the hydroazidation of 4-phenylbutene (1).

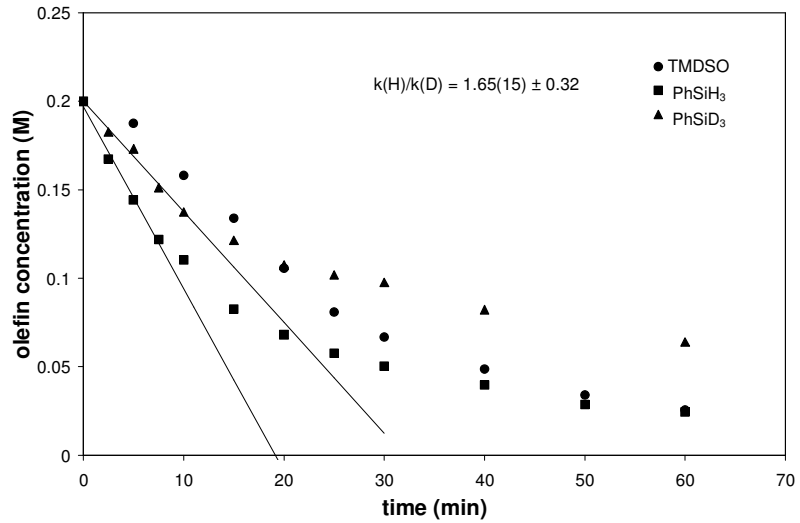

4.3.3. Kinetics Data

\section{Hydrohydrazination Reaction}

Standard Conditions Standard conditions: 4-phenyl butene (1) (19 $\mu \mathrm{L}, 0.13 \mathrm{mmol}, 0.17 \mathrm{M}, 1.0$ equiv); catalyst 20 (2 mg, $0.005 \mathrm{mmol}, 0.007 \mathrm{M}, 0.04$ equiv); azodicarboxylate 2 (43 mg, $0.20 \mathrm{mmol}, 0.27 \mathrm{M}, 1.5$ equiv); phenylsilane ( $16 \mu \mathrm{L}, 0.13 \mathrm{mmol}$, $0.17 \mathrm{M}, 1.0$ equiv); $25^{\circ} \mathrm{C} ; \mathrm{CD}_{3} \mathrm{OD}(0.75 \mathrm{~mL})$. 


\begin{tabular}{ccc}
\multicolumn{3}{c}{ standard conditions } \\
\hline time(min) & $\begin{array}{c}\text { integral ratio } \\
\mathbf{3 / 1}\end{array}$ & $\begin{array}{c}\text { concentration } \mathbf{1} \\
\text { (M) }\end{array}$ \\
\hline 0 & 0.00 & 0.173 \\
15 & 0.03 & 0.146 \\
25 & 0.19 & 0.126 \\
35 & 0.38 & 0.116 \\
45 & 0.49 & 0.101 \\
55 & 0.71 & 0.086 \\
65 & 1.03 & 0.080 \\
75 & 1.16 & 0.066 \\
85 & 1.63 & 0.065 \\
95 & 1.68 & 0.061 \\
105 & 1.84 & 0.049 \\
115 & 2.57 & 0.047 \\
125 & 2.70 & 0.045 \\
135 & 2.85 & 0.038 \\
\hline reaction rate r: & $0.00168(16) \pm 0.00034$ \\
\hline
\end{tabular}

Variation of the concentration of 4-phenylbutene (1) (Figure 1)

standard conditions, concentration $\mathbf{1}=0.10 \mathrm{M}$

\begin{tabular}{ccc}
\hline time(min) & $\begin{array}{c}\text { integral ratio } \\
\mathbf{3 / 1}\end{array}$ & $\begin{array}{c}\text { concentration } \mathbf{1} \\
(\mathrm{M})\end{array}$ \\
\hline 0 & 0.00 & 0.097 \\
10 & 0.08 & 0.089 \\
20 & 0.22 & 0.076 \\
30 & 0.37 & 0.074 \\
40 & 0.53 & 0.068 \\
50 & 0.61 & 0.063 \\
60 & 0.98 & 0.054 \\
70 & 0.98 & 0.052 \\
80 & 1.28 & 0.048 \\
90 & 1.20 & 0.047 \\
100 & 1.68 & 0.040 \\
110 & 2.34 & 0.034 \\
120 & 2.25 & 0.035 \\
160 & 4.30 & 0.022 \\
\hline reaction rate r: & $0.00083(15) \pm 0.00031$ \\
\hline
\end{tabular}

standard conditions, concentration $1=0.25 \mathrm{M}$

\begin{tabular}{ccc}
\hline time (min) & $\begin{array}{c}\text { integral ratio } \\
\mathbf{3 / 1}\end{array}$ & $\begin{array}{c}\text { concentration } \mathbf{1} \\
(\mathrm{M})\end{array}$ \\
\hline 0 & 0.00 & 0.247 \\
10 & 0.15 & 0.228 \\
20 & 0.37 & 0.194 \\
30 & 0.57 & 0.178 \\
40 & 0.78 & 0.167 \\
50 & 1.01 & 0.152 \\
60 & 1.38 & 0.142 \\
70 & 1.58 & 0.133 \\
80 & 1.96 & 0.120 \\
90 & 2.35 & 0.110 \\
100 & 2.71 & 0.104 \\
110 & 3.16 & 0.096 \\
120 & 3.21 & 0.095 \\
160 & 4.17 & 0.081 \\
\hline reaction rate $\mathrm{r}:$ & $0.0024(2) \pm 0.0005$ \\
\hline
\end{tabular}

van't Hoff reaction order

\begin{tabular}{cccc}
\hline $\mathrm{x}=\log (\mathrm{c})$ & $\mathrm{y}=\log (\mathrm{r})$ & $\mathrm{s}_{\mathrm{yi}}$ & $\mathrm{v}_{\mathrm{yi}}$ \\
\hline-1.01 & -3.08 & -0.08 & -0.16 \\
-0.88 & -2.94 & -0.05 & -0.11 \\
-0.76 & -2.78 & -0.04 & -0.09 \\
-0.61 & -2.62 & -0.04 & -0.09 \\
-0.46 & -2.57 & -0.10 & -0.22 \\
\hline
\end{tabular}

standard conditions, concentration $\mathbf{1}=0.13 \mathrm{M}$

\begin{tabular}{ccc}
\hline time(min) & $\begin{array}{c}\text { integral ratio } \\
\mathbf{3 / 1}\end{array}$ & $\begin{array}{c}\text { concentration } \mathbf{1} \\
(\mathrm{M})\end{array}$ \\
\hline 0 & 0.00 & 0.132 \\
10 & 0.31 & 0.116 \\
20 & 0.49 & 0.105 \\
30 & 0.81 & 0.098 \\
40 & 1.08 & 0.088 \\
50 & 1.61 & 0.076 \\
60 & 1.86 & 0.067 \\
70 & 2.34 & 0.061 \\
80 & 2.86 & 0.055 \\
90 & 3.65 & 0.049 \\
100 & 5.07 & 0.042 \\
110 & 4.92 & 0.041 \\
\hline reaction rate r: & $0.00114(13) \pm 0.00027$ \\
\hline
\end{tabular}

standard conditions, concentration $\mathbf{1}=0.35 \mathrm{M}$

\begin{tabular}{ccc}
\hline time(min) & $\begin{array}{c}\text { integral ratio } \\
\mathbf{3 / 1}\end{array}$ & $\begin{array}{c}\text { concentration } \mathbf{1} \\
(\mathrm{M})\end{array}$ \\
\hline 0 & 0.00 & 0.344 \\
10 & 0.10 & 0.292 \\
20 & 0.24 & 0.276 \\
30 & 0.44 & 0.260 \\
40 & 0.60 & 0.231 \\
50 & 0.79 & 0.215 \\
60 & 0.88 & 0.207 \\
70 & 1.12 & 0.204 \\
80 & 1.38 & 0.183 \\
90 & 1.53 & 0.186 \\
100 & 1.50 & 0.162 \\
110 & 1.76 & 0.176 \\
120 & 2.26 & 0.179 \\
160 & 2.46 & 0.155 \\
\hline reaction rate r: & $0.0026(6) \pm 0.0013$ \\
\hline
\end{tabular}

\begin{tabular}{|c|c|}
\hline reaction order: & $0.98(16) \pm 0.23$ \\
\hline & $R=0.984$ \\
\hline
\end{tabular}


Variation of the concentration of azodicarboxylate 2 (Figure 2)

standard conditions, concentration $2=0.093 \mathrm{M}$

\begin{tabular}{ccc}
\hline time(min) & $\begin{array}{c}\text { integral ratio } \\
\mathbf{3 / 1}\end{array}$ & $\begin{array}{c}\text { concentration } \mathbf{1} \\
(\mathrm{M})\end{array}$ \\
\hline 0 & 0.00 & 0.170 \\
10 & 0.15 & 0.151 \\
20 & 0.28 & 0.136 \\
30 & 0.51 & 0.122 \\
40 & 0.58 & 0.112 \\
50 & 0.78 & 0.104 \\
60 & 0.84 & 0.099 \\
70 & 0.75 & 0.099 \\
80 & 0.85 & 0.099 \\
90 & 0.78 & 0.098 \\
100 & 0.80 & 0.099 \\
\hline reaction rate r: & $0.00158(8) \pm 0.00018$ \\
\hline \multicolumn{3}{c}{} \\
\hline
\end{tabular}

standard conditions, concentration $2=0.20 \mathrm{M}$

\begin{tabular}{ccc}
\hline time(min) & $\begin{array}{c}\text { integral ratio } \\
\mathbf{3} \mathbf{1}\end{array}$ & $\begin{array}{c}\text { concentration } \mathbf{1} \\
(\mathrm{M})\end{array}$ \\
\hline 0 & 0.00 & 0.170 \\
10 & 0.10 & 0.153 \\
20 & 0.27 & 0.140 \\
38 & 0.55 & 0.120 \\
42 & 0.67 & 0.118 \\
50 & 0.86 & 0.106 \\
60 & 1.11 & 0.096 \\
70 & 1.05 & 0.094 \\
80 & 1.58 & 0.082 \\
90 & 1.71 & 0.075 \\
100 & 2.14 & 0.070 \\
110 & 2.36 & 0.065 \\
\hline reaction rate r: & $0.00129(10) \pm 0.00022$ \\
\hline
\end{tabular}

standard conditions, concentration $2=0.16 \mathrm{M}$

\begin{tabular}{ccc}
\hline time(min) & $\begin{array}{c}\text { integral ratio } \\
\mathbf{3 / 1}\end{array}$ & $\begin{array}{c}\text { concentration } \mathbf{1} \\
(\mathrm{M})\end{array}$ \\
\hline 0 & 0.00 & 0.170 \\
10 & 0.17 & 0.149 \\
20 & 0.37 & 0.131 \\
30 & 0.50 & 0.119 \\
40 & 0.75 & 0.112 \\
50 & 1.00 & 0.099 \\
60 & 1.30 & 0.091 \\
70 & 1.20 & 0.090 \\
80 & 1.48 & 0.079 \\
90 & 1.86 & 0.072 \\
100 & 1.89 & 0.068 \\
110 & 2.10 & 0.065 \\
\hline reaction rate r: & \multicolumn{2}{|c}{$0.00170(16) \pm 0.0003$} \\
\hline
\end{tabular}

standard conditions, concentration $2=0.33 \mathrm{M}$

\begin{tabular}{ccc}
\hline time(min) & $\begin{array}{c}\text { integral ratio } \\
\mathbf{3} \mathbf{1}\end{array}$ & $\begin{array}{c}\text { concentration } \mathbf{1} \\
(\mathrm{M})\end{array}$ \\
\hline 0 & 0.00 & 0.170 \\
10 & 0.15 & 0.146 \\
20 & 0.23 & 0.137 \\
30 & 0.44 & 0.130 \\
40 & 0.67 & 0.121 \\
50 & 0.92 & 0.109 \\
60 & 1.09 & 0.094 \\
70 & 1.20 & 0.088 \\
80 & 1.49 & 0.078 \\
90 & 1.65 & 0.073 \\
100 & 1.95 & 0.070 \\
110 & 2.53 & 0.060 \\
\hline reaction rate r: & \multicolumn{2}{c}{$0.0012(3) \pm 0.006$} \\
\hline
\end{tabular}

van't Hoff reaction order

\begin{tabular}{cccc}
\hline $\mathrm{x}=\log (\mathrm{c})$ & $\mathrm{y}=\log (\mathrm{r})$ & $\mathrm{s}_{\mathrm{yi}}$ & $\mathrm{v}_{\mathrm{yi}}$ \\
\hline-1.03 & -2.80 & -0.02 & -0.05 \\
-0.80 & -2.77 & -0.04 & -0.09 \\
-0.70 & -2.89 & -0.03 & -0.07 \\
-0.57 & -2.80 & -0.04 & -0.08 \\
-0.48 & -2.92 & -0.11 & -0.23
\end{tabular}

\begin{tabular}{|l|l}
\hline reaction order: & $-0.18(8) \pm 0.12$ \\
& $R=-0.412$
\end{tabular}


Variation of the concentration of phenylsilane (Figure 3)

standard conditions, concentration $\mathrm{PhSiH}_{3}=0.07 \mathrm{M}$

\begin{tabular}{ccc}
\hline time(min) & $\begin{array}{c}\text { integral ratio } \\
\mathbf{3} / \mathbf{1}\end{array}$ & $\begin{array}{c}\text { concentration } \mathbf{1} \\
(\mathrm{M})\end{array}$ \\
\hline 0 & 0.01 & 0.170 \\
6 & 0.03 & 0.167 \\
12 & 0.06 & 0.156 \\
18 & 0.11 & 0.154 \\
24 & 0.15 & 0.146 \\
35 & 0.23 & 0.144 \\
41 & 0.30 & 0.140 \\
47 & 0.35 & 0.135 \\
53 & 0.38 & 0.139 \\
\hline reaction rate r: & $0.00102(13) \pm 0.00018$ \\
\hline
\end{tabular}

standard conditions, concentration $\mathrm{PhSiH}_{3}=0.21 \mathrm{M}$

\begin{tabular}{ccc}
\hline time(min) & $\begin{array}{c}\text { integral ratio } \\
\mathbf{3} / \mathbf{1}\end{array}$ & $\begin{array}{c}\text { concentration } \mathbf{1} \\
(\mathrm{M})\end{array}$ \\
\hline 0 & 0.02 & 0.170 \\
6 & 0.07 & 0.159 \\
12 & 0.20 & 0.143 \\
18 & 0.30 & 0.135 \\
24 & 0.41 & 0.125 \\
35 & 0.65 & 0.113 \\
41 & 0.81 & 0.105 \\
47 & 0.99 & 0.095 \\
53 & 1.17 & 0.090 \\
\hline reaction rate r: & $0.00166(13) \pm 0.00015$ \\
\hline
\end{tabular}

van't Hoff reaction order

\begin{tabular}{|c|c|c|c|}
\hline$x=\log (c)$ & $y=\log (r)$ & $\mathrm{s}_{\mathrm{yi}}$ & $v_{y i}$ \\
\hline-1.15 & -2.99 & -0.05 & -0.12 \\
\hline-0.85 & -2.82 & -0.08 & -0.11 \\
\hline-0.77 & -2.80 & -0.04 & -0.05 \\
\hline-0.68 & -2.78 & -0.03 & -0.04 \\
\hline-0.59 & -2.76 & -0.010 & -0.011 \\
\hline \multirow[t]{3}{*}{-0.47} & -2.74 & -0.019 & -0.021 \\
\hline & reaction order: & \multicolumn{2}{|c|}{$0.22(11) \pm 0.16$} \\
\hline & & \multicolumn{2}{|c|}{$R=0.999$} \\
\hline
\end{tabular}

standard conditions, concentration $\mathrm{PhSiH}_{3}=0.14 \mathrm{M}$

\begin{tabular}{ccc}
\hline time $(\mathrm{min})$ & $\begin{array}{c}\text { integral ratio } \\
\mathbf{3 / 1}\end{array}$ & $\begin{array}{c}\text { concentration } \mathbf{1} \\
(\mathrm{M})\end{array}$ \\
\hline 0 & 0.00 & 0.170 \\
10 & 0.19 & 0.143 \\
26 & 0.38 & 0.123 \\
33 & 0.57 & 0.119 \\
40 & 0.65 & 0.114 \\
50 & 0.73 & 0.104 \\
60 & 0.98 & 0.096 \\
70 & 1.09 & 0.092 \\
80 & 1.37 & 0.077 \\
90 & 1.53 & 0.077 \\
100 & 1.89 & 0.070 \\
\hline reaction rate r: & $0.00151(26) \pm 0.0005$
\end{tabular}

standard conditions, concentration $\mathrm{PhSiH}_{3}=0.26 \mathrm{M}$

\begin{tabular}{ccc}
\hline time(min) & $\begin{array}{c}\text { integral ratio } \\
\mathbf{3} \mathbf{1}\end{array}$ & $\begin{array}{c}\text { concentration } \mathbf{1} \\
(\mathrm{M})\end{array}$ \\
\hline 0 & 0.03 & 0.170 \\
6 & 0.09 & 0.160 \\
12 & 0.18 & 0.148 \\
18 & 0.28 & 0.139 \\
24 & 0.39 & 0.127 \\
32 & 0.60 & 0.115 \\
38 & 0.73 & 0.109 \\
44 & 0.85 & 0.103 \\
50 & 1.04 & 0.094 \\
56 & 1.20 & 0.090 \\
\hline \multirow{2}{*}{ reaction rate r: } & $0.00174(4) \pm 0.00005$ \\
\hline \multicolumn{2}{c}{} \\
\hline
\end{tabular}

standard conditions, concentration $\mathrm{PhSiH}_{3}=0.34 \mathrm{M}$

\begin{tabular}{ccc}
\hline time(min) & $\begin{array}{c}\text { integral ratio } \\
\mathbf{3} / \mathbf{1}\end{array}$ & $\begin{array}{c}\text { concentration } \mathbf{1} \\
(\mathrm{M})\end{array}$ \\
\hline 0 & 0.04 & 0.170 \\
6 & 0.11 & 0.155 \\
12 & 0.18 & 0.144 \\
18 & 0.31 & 0.133 \\
24 & 0.43 & 0.125 \\
30 & 0.55 & 0.113 \\
36 & 0.70 & 0.103 \\
42 & 0.85 & 0.097 \\
48 & 1.04 & 0.088 \\
54 & 1.20 & 0.082 \\
\hline reaction rate r: & $0.00183(8) \pm 0.00009$
\end{tabular}


Variation of the concentration of catalyst 20 (Figure 4)

\begin{tabular}{ccc}
\multicolumn{3}{c}{ standard conditions, concentration $\mathbf{2 0}=0.010 \mathrm{M}$} \\
\hline time(min) & $\begin{array}{c}\text { integral ratio } \\
\mathbf{3 / 1}\end{array}$ & $\begin{array}{c}\text { concentration } \mathbf{1} \\
(\mathrm{M})\end{array}$ \\
\hline 0 & 0.04 & 0.170 \\
6 & 0.09 & 0.159 \\
12 & 0.20 & 0.147 \\
18 & 0.29 & 0.139 \\
24 & 0.39 & 0.131 \\
30 & 0.52 & 0.118 \\
36 & 0.62 & 0.111 \\
42 & 0.72 & 0.106 \\
48 & 0.85 & 0.099 \\
54 & 1 & 0.092 \\
\hline reaction rate r: & $0.00167(5) \pm 0.00007$ \\
\hline
\end{tabular}

van't Hoff reaction order

\begin{tabular}{cccc}
\hline $\mathrm{x}=\log (\mathrm{c})$ & $\mathrm{y}=\log (\mathrm{r})$ & $\mathrm{s}_{\mathrm{yi}}$ & $\mathrm{V}_{\mathrm{yi}}$ \\
\hline-2.17 & -2.80 & -0.04 & -0.08 \\
-2.00 & -2.78 & -0.015 & -0.017 \\
-1.89 & -2.65 & -0.04 & -0.04 \\
-1.77 & -2.60 & -0.03 & -0.03 \\
\hline
\end{tabular}

reaction order: $\quad 0.54(10) \pm 0.22$

$R=0.926$ standard conditions, concentration $20=0.013 \mathrm{M}$

\begin{tabular}{ccc}
\hline time(min) & $\begin{array}{c}\text { integral ratio } \\
\mathbf{3 / 1}\end{array}$ & $\begin{array}{c}\text { concentration } \mathbf{1} \\
(\mathrm{M})\end{array}$ \\
\hline 0 & 0.06 & 0.170 \\
6 & 0.12 & 0.144 \\
12 & 0.40 & 0.135 \\
18 & 0.60 & 0.121 \\
24 & 0.78 & 0.110 \\
30 & 1.06 & 0.100 \\
36 & 1.29 & 0.090 \\
42 & 1.54 & 0.082 \\
48 & 1.82 & 0.075 \\
54 & 2.08 & 0.068 \\
\hline reaction rate r: & $0.00223(19) \pm 0.00023$ \\
\hline
\end{tabular}

standard conditions, concentration $20=0.017 \mathrm{M}$

\begin{tabular}{ccc}
\hline time(min) & $\begin{array}{c}\text { integral ratio } \\
\mathbf{3 / 1}\end{array}$ & $\begin{array}{c}\text { concentration } \mathbf{1} \\
(\mathrm{M})\end{array}$ \\
\hline 0 & 0.07 & 0.170 \\
6 & 0.24 & 0.148 \\
12 & 0.42 & 0.132 \\
18 & 0.61 & 0.116 \\
24 & 0.83 & 0.104 \\
30 & 1.09 & 0.093 \\
36 & 1.33 & 0.084 \\
42 & 1.59 & 0.076 \\
48 & 1.91 & 0.069 \\
54 & 2.12 & 0.063 \\
\hline reaction rate r: & $0.00254(16) \pm 0.00018$ \\
\hline
\end{tabular}


Variation of the temperature (Figure 6)

\begin{tabular}{ccc}
\multicolumn{3}{c}{ standard conditions, $\mathrm{T}=296 \mathrm{~K}$} \\
\hline time $(\mathrm{min})$ & $\begin{array}{c}\text { integral ratio } \\
\mathbf{3 / 1}\end{array}$ & $\begin{array}{c}\text { concentration } \mathbf{1} \\
(\mathrm{M})\end{array}$ \\
\hline 0 & 0.00 & 0.170 \\
2 & 0.06 & 0.160 \\
13 & 0.60 & 0.106 \\
23 & 1.05 & 0.083 \\
40 & 2.50 & 0.049 \\
55 & 3.58 & 0.037 \\
74 & 4.67 & 0.030 \\
88 & 5.66 & 0.026 \\
116 & 6.04 & 0.024 \\
\hline \multirow{2}{*}{ reaction rate $\mathrm{r}:$} & $0.0039(5) \pm 0.0010$
\end{tabular}

standard conditions, $\mathrm{T}=308 \mathrm{~K}$

\begin{tabular}{ccc}
\hline time(min) & $\begin{array}{c}\text { integral ratio } \\
\mathbf{3 / 1}\end{array}$ & $\begin{array}{c}\text { concentration } \mathbf{1} \\
(\mathrm{M})\end{array}$ \\
\hline 0 & 0.00 & 0.170 \\
2 & 0.11 & 0.153 \\
4 & 0.35 & 0.125 \\
6 & 0.55 & 0.110 \\
8 & 0.73 & 0.098 \\
10 & 0.98 & 0.086 \\
12 & 1.11 & 0.080 \\
14 & 1.54 & 0.067 \\
16 & 1.83 & 0.060 \\
18 & 2.08 & 0.055 \\
\hline reaction rate $\mathrm{r}:$ & $0.0104(8) \pm 0.0017$ \\
\hline
\end{tabular}

\begin{tabular}{cccc}
\multicolumn{4}{c}{ Arrhenius plot } \\
\hline $\mathrm{x}=1 / \mathrm{T}$ & $\mathrm{y}=\ln (\mathrm{r})$ & $\mathrm{s}_{\mathrm{yi}}$ & $\mathrm{v}_{\mathrm{yi}}$ \\
\hline 0.00336 & -5.55 & -0.12 & -0.25 \\
0.00330 & -5.07 & -0.012 & -0.03 \\
0.00325 & -4.57 & -0.08 & -0.16 \\
0.00319 & -4.09 & -0.13 & -0.27 \\
\hline
\end{tabular}

standard conditions, $\mathrm{T}=303 \mathrm{~K}$

\begin{tabular}{ccc}
\hline time(min) & $\begin{array}{c}\text { integral ratio } \\
\mathbf{3 / 1}\end{array}$ & $\begin{array}{c}\text { concentration } \mathbf{1} \\
(\mathrm{M})\end{array}$ \\
\hline 0 & 0.00 & 0.170 \\
2 & 0.08 & 0.157 \\
6 & 0.28 & 0.133 \\
11 & 0.69 & 0.101 \\
14 & 0.97 & 0.086 \\
17 & 0.94 & 0.088 \\
20 & 1.50 & 0.068 \\
23 & 1.71 & 0.063 \\
26 & 2.09 & 0.055 \\
29 & 2.33 & 0.051 \\
\hline reaction rate $\mathrm{r}:$ & $0.0064(4) \pm 0.0010$ \\
\hline
\end{tabular}

standard conditions, $\mathrm{T}=313 \mathrm{~K}$

\begin{tabular}{ccc}
\hline time(min) & $\begin{array}{c}\text { integral ratio } \\
\mathbf{3 / 1}\end{array}$ & $\begin{array}{c}\text { concentration } \mathbf{1} \\
(\mathrm{M})\end{array}$ \\
\hline 0 & 0.00 & 0.170 \\
1.5 & 0.27 & 0.134 \\
3 & 0.54 & 0.111 \\
4.5 & 0.80 & 0.094 \\
7 & 1.29 & 0.074 \\
8.5 & 1.76 & 0.062 \\
10 & 2.17 & 0.054 \\
11.5 & 2.22 & 0.053 \\
13 & 2.39 & 0.050 \\
14.5 & 2.70 & 0.046 \\
\hline
\end{tabular}

reaction rate $\mathrm{r}: \quad 0.0167(21) \pm 0.0045$

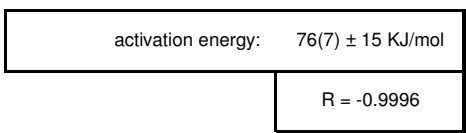

\section{Kinetic isotope effect (Figure 7)}

standard conditions, with $\mathrm{PhSiD}_{3}$

\begin{tabular}{ccc}
\hline time(min) & $\begin{array}{c}\text { integral ratio } \\
\mathbf{2 7 / 1}\end{array}$ & $\begin{array}{c}\text { concentration } \mathbf{1} \\
(\mathrm{M})\end{array}$ \\
\hline 10 & 0.07 & 0.159 \\
20 & 0.10 & 0.155 \\
30 & 0.19 & 0.143 \\
40 & 0.22 & 0.139 \\
50 & 0.31 & 0.130 \\
60 & 0.41 & 0.120 \\
70 & 0.50 & 0.113 \\
80 & 0.52 & 0.112 \\
90 & 0.57 & 0.108 \\
100 & 0.74 & 0.098 \\
110 & 0.70 & 0.100 \\
120 & 0.75 & 0.097 \\
130 & 0.84 & 0.092 \\
195 & 1.46 & 0.069 \\
\hline reaction rate $\mathrm{r}:$ & $0.00168(16) \pm 0.00034$ \\
\hline
\end{tabular}

kinetic isotope effect: $\quad 2.2(3) \pm 0.5$

\section{Hydroazidation Reaction}

Standard Conditions Standard conditions: 4-phenylbutene (1) $\left(75 \mu \mathrm{L}, 0.50 \mathrm{mmol}, 0.20 \mathrm{M}, 1.0\right.$ equiv); $\mathrm{Co}\left(\mathrm{BF}_{4}\right)_{2} \bullet 6 \mathrm{H}_{2} \mathrm{O}(10 \mathrm{mg}$, $0.030 \mathrm{mmol}, 0.012 \mathrm{M}, 0.060$ equiv); ligand 43 (14 mg, $0.030 \mathrm{mmol}, 0.012 \mathrm{M}, 0.060$ equiv); tert-butyl hydroperoxide (25 $\mu$ l, 0.14 
mmol, 0. $056 \mathrm{M}, 0.28$ equiv); tosylazide (203) $(0.23 \mathrm{~mL}, 1.5 \mathrm{mmol}, 0.60 \mathrm{M}, 3.0$ equiv); TMDSO (0.18 mL, $1.0 \mathrm{mmol}, 0.40 \mathrm{M}$, 2.0 equiv); $23^{\circ} \mathrm{C} ; \mathrm{EtOH}(2.5 \mathrm{~mL})$.

\begin{tabular}{ccc}
\multicolumn{3}{c}{ standard conditions } \\
\hline time(min) & $\begin{array}{c}\text { integral ratio } \\
\mathbf{3 3 / 1}\end{array}$ & $\begin{array}{c}\text { concentration } \mathbf{1} \\
(\mathrm{M})\end{array}$ \\
\hline 5 & 0.07 & 0.187 \\
10 & 0.26 & 0.158 \\
15 & 0.48 & 0.134 \\
20 & 0.87 & 0.106 \\
25 & 1.44 & 0.081 \\
30 & 1.95 & 0.067 \\
40 & 3.02 & 0.049 \\
50 & 4.75 & 0.034 \\
60 & 6.63 & 0.026 \\
70 & 8.26 & 0.021 \\
90 & 13.75 & 0.013 \\
\hline reaction rate r: & $0.00539(14) \pm 0.0003$ \\
\end{tabular}

Variation of the concentration of 4-phenylbutene (1) (Figure 8)

\begin{tabular}{ccc}
\multicolumn{3}{c}{ standard conditions, concentration $\mathbf{1}=0.10 \mathrm{M}$} \\
\hline time(min) & $\begin{array}{c}\text { integral ratio } \\
\mathbf{3 3 / 1}\end{array}$ & $\begin{array}{c}\text { concentration } \mathbf{1} \\
(\mathrm{M})\end{array}$ \\
\hline 2.5 & 0.07 & 0.093 \\
5 & 0.14 & 0.088 \\
7.5 & 0.34 & 0.074 \\
10 & 0.57 & 0.063 \\
15 & 1.37 & 0.042 \\
20 & 2.82 & 0.026 \\
25 & 5.32 & 0.015 \\
30 & 8.87 & 0.010 \\
40 & 22.58 & 0.004 \\
50 & 74.45 & 0.001 \\
60 & 363.08 & 0.000 \\
\hline reaction rate r: & $0.00411(21) \pm 0.0003$ \\
\hline \multicolumn{3}{l}{}
\end{tabular}

van't Hoff reaction order

\begin{tabular}{cccc}
\hline $\mathrm{x}=\log (\mathrm{c})$ & $\mathrm{y}=\log (\mathrm{r})$ & $\mathrm{s}_{\mathrm{yi}}$ & $\mathrm{v}_{\mathrm{yi}}$ \\
\hline-1.00 & -2.39 & -0.023 & -0.05 \\
-0.70 & -2.27 & -0.011 & -0.016 \\
-0.52 & -2.16 & -0.015 & -0.022 \\
-0.40 & -2.14 & -0.009 & -0.012 \\
\hline
\end{tabular}

reaction order: $\quad 0.45(3) \pm 0.07$

$R=0.990$ standard conditions, concentration $\mathbf{1}=0.30 \mathrm{M}$

\begin{tabular}{ccc}
\hline time(min) & $\begin{array}{c}\text { integral ratio } \\
\mathbf{3 3 / 1}\end{array}$ & $\begin{array}{c}\text { concentration } \mathbf{1} \\
(\mathrm{M})\end{array}$ \\
\hline 2.5 & 0.02 & 0.293 \\
5 & 0.05 & 0.284 \\
7.5 & 0.11 & 0.270 \\
10 & 0.20 & 0.250 \\
15 & 0.43 & 0.209 \\
20 & 0.67 & 0.178 \\
25 & 0.90 & 0.156 \\
30 & 1.12 & 0.139 \\
40 & 1.56 & 0.115 \\
50 & 1.99 & 0.099 \\
60 & 2.46 & 0.085 \\
75 & 2.96 & 0.074 \\
90 & 3.78 & 0.061 \\
\hline reaction rate r: & $0.00729(25) \pm 0.0004$
\end{tabular}

standard conditions, concentration $1=0.40 \mathrm{M}$

\begin{tabular}{ccc}
\hline time(min) & $\begin{array}{c}\text { integral ratio } \\
\mathbf{3 3 / 1}\end{array}$ & $\begin{array}{c}\text { concentration } \mathbf{1} \\
(\mathrm{M})\end{array}$ \\
\hline 2.5 & 0.01 & 0.395 \\
5 & 0.04 & 0.386 \\
7.5 & 0.08 & 0.371 \\
10 & 0.15 & 0.348 \\
15 & 0.28 & 0.310 \\
20 & 0.46 & 0.271 \\
25 & 0.58 & 0.251 \\
30 & 0.69 & 0.235 \\
40 & 0.78 & 0.222 \\
50 & 1.04 & 0.194 \\
60 & 1.24 & 0.176 \\
75 & 1.48 & 0.159 \\
90 & 1.49 & 0.158 \\
\hline reaction rate r: & $0.00774(15) \pm 0.00021$ \\
\hline
\end{tabular}


Variation of the concentration of tosylazide (203) (Figure 9)

standard conditions, concentration $\mathrm{TsN}_{3}=0.30 \mathrm{M}$

\begin{tabular}{ccc}
\hline time(min) & $\begin{array}{c}\text { integral ratio } \\
\mathbf{3 3 / 1}\end{array}$ & $\begin{array}{c}\text { concentration } \mathbf{1} \\
(\mathrm{M})\end{array}$ \\
\hline 2.5 & 0.03 & 0.193 \\
5 & 0.07 & 0.186 \\
7.5 & 0.16 & 0.171 \\
10 & 0.26 & 0.158 \\
15 & 0.44 & 0.137 \\
20 & 0.67 & 0.118 \\
25 & 0.90 & 0.104 \\
30 & 1.16 & 0.091 \\
40 & 1.60 & 0.076 \\
50 & 1.93 & 0.067 \\
60 & 2.77 & 0.052 \\
75 & 3.70 & 0.042 \\
90 & 4.27 & 0.037 \\
\hline \multirow{2}{*}{ reaction rate r: } & $0.00562(26) \pm 0.0019$ \\
\hline \multicolumn{2}{c}{} \\
\hline
\end{tabular}

van't Hoff reaction order

\begin{tabular}{cccc}
\hline $\mathrm{x}=\log (\mathrm{c})$ & $\mathrm{y}=\log (\mathrm{r})$ & $\mathrm{s}_{\mathrm{yi}}$ & $\mathrm{v}_{\mathrm{yi}}$ \\
\hline-0.22 & -2.27 & -0.011 & -0.02 \\
-0.52 & -2.25 & -0.020 & -0.145 \\
-0.70 & -2.30 & -0.013 & -0.096 \\
\hline
\end{tabular}

Variation of the concentration of TMDSO (Figure 10)

standard conditions, concentration TMDSO $=0.10 \mathrm{M}$

\begin{tabular}{ccc}
\hline time(min) & $\begin{array}{c}\text { integral ratio } \\
\mathbf{3 3 / 1}\end{array}$ & $\begin{array}{c}\text { concentration } \mathbf{1} \\
(\mathrm{M})\end{array}$ \\
\hline 2.5 & 0.00 & 0.199 \\
5 & 0.01 & 0.198 \\
7.5 & 0.02 & 0.195 \\
10 & 0.05 & 0.190 \\
15 & 0.12 & 0.178 \\
20 & 0.23 & 0.161 \\
25 & 0.39 & 0.143 \\
30 & 0.52 & 0.130 \\
40 & 0.79 & 0.111 \\
50 & 1.05 & 0.096 \\
60 & 1.32 & 0.085 \\
75 & 1.41 & 0.082 \\
90 & 1.74 & 0.072 \\
\hline reaction rate r: & $0.00319(19) \pm 0.0004$ \\
\hline
\end{tabular}

van't Hoff reaction order

\begin{tabular}{cccc}
\hline $\mathrm{x}=\log (\mathrm{c})$ & $\mathrm{y}=\log (\mathrm{r})$ & $\mathrm{S}_{\mathrm{yi}}$ & $\mathrm{v}_{\mathrm{yi}}$ \\
\hline-0.22 & -2.21 & -0.008 & -0.01 \\
-0.40 & -2.27 & -0.011 & -0.024 \\
-0.70 & -2.27 & -0.053 & -0.114 \\
-1.00 & -2.50 & -0.026 & -0.055 \\
\hline \multicolumn{3}{c}{} \\
\cline { 2 - 3 } & \multicolumn{2}{c}{$0.33(3) \pm 0.10$} \\
\cline { 2 - 3 } & \multicolumn{2}{c}{$\mathrm{R}=0.982$} \\
\hline
\end{tabular}

standard conditions, concentration $\mathrm{TsN}_{3}=0.20 \mathrm{M}$

\begin{tabular}{ccc}
\hline time(min) & $\begin{array}{c}\text { integral ratio } \\
\mathbf{3 3 / 1}\end{array}$ & $\begin{array}{c}\text { concentration } \mathbf{1} \\
(\mathrm{M})\end{array}$ \\
\hline 2.5 & 0.04 & 0.193 \\
5 & 0.07 & 0.186 \\
7.5 & 0.15 & 0.173 \\
10 & 0.24 & 0.161 \\
15 & 0.34 & 0.148 \\
20 & 0.49 & 0.133 \\
25 & 0.62 & 0.122 \\
30 & 0.74 & 0.114 \\
40 & 0.90 & 0.104 \\
52 & 1.19 & 0.090 \\
60 & 1.16 & 0.091 \\
75 & 1.27 & 0.087 \\
90 & 1.57 & 0.077 \\
\hline reaction rate r: & $0.00499(15) \pm 0.0011$ \\
\hline
\end{tabular}

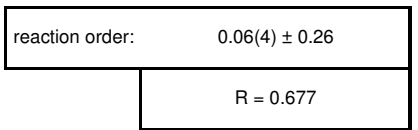

standard conditions, concentration TMDSO $=0.20 \mathrm{M}$

\begin{tabular}{ccc}
\hline time(min) & $\begin{array}{c}\text { integral ratio } \\
\mathbf{3 3 / 1}\end{array}$ & $\begin{array}{c}\text { concentration } \mathbf{1} \\
(\mathrm{M})\end{array}$ \\
\hline 2.5 & 0.01 & 0.198 \\
5 & 0.03 & 0.195 \\
7.5 & 0.07 & 0.187 \\
10 & 0.15 & 0.173 \\
15 & 0.39 & 0.143 \\
20 & 0.64 & 0.120 \\
25 & 0.95 & 0.101 \\
30 & 1.47 & 0.080 \\
40 & 2.02 & 0.065 \\
50 & 2.71 & 0.053 \\
60 & 3.82 & 0.041 \\
75 & 5.05 & 0.032 \\
90 & 5.82 & 0.029 \\
\hline reaction rate r: & $0.0054(6) \pm 0.0013$ \\
\hline
\end{tabular}

standard conditions, concentration TMDSO $=0.60 \mathrm{M}$

\begin{tabular}{ccc}
\hline time(min) & $\begin{array}{c}\text { integral ratio } \\
\mathbf{3 3 / 1}\end{array}$ & $\begin{array}{c}\text { concentration } \mathbf{1} \\
(\mathrm{M})\end{array}$ \\
\hline 2.5 & 0.03 & 0.195 \\
5 & 0.09 & 0.183 \\
7.5 & 0.19 & 0.167 \\
10 & 0.34 & 0.149 \\
15 & 0.67 & 0.119 \\
20 & 1.24 & 0.088 \\
25 & 1.87 & 0.068 \\
30 & 2.60 & 0.054 \\
40 & 4.63 & 0.035 \\
50 & 7.58 & 0.023 \\
60 & 10.22 & 0.017 \\
75 & 7.86 & 0.022 \\
90 & 10.91 & 0.016 \\
\hline reaction rate r: & $0.00622(11) \pm 0.00013$ \\
\hline
\end{tabular}


Variation of the concentration of $\mathrm{Co}\left(\mathrm{BF}_{4}\right)_{2} \cdot 6 \mathrm{H}_{2} \mathrm{O} /$ Ligand 43 (Figure 11)

\begin{tabular}{ccc}
\multicolumn{3}{c}{ standard conditions, concentration catalyst $=0.003 \mathrm{M}$} \\
\hline time(min) & $\begin{array}{c}\text { integral ratio } \\
\mathbf{3 3 / 1}\end{array}$ & $\begin{array}{c}\text { concentration } \mathbf{1} \\
(\mathrm{M})\end{array}$ \\
\hline 5 & 0.01 & 0.199 \\
10 & 0.01 & 0.197 \\
20 & 0.07 & 0.187 \\
30 & 0.18 & 0.169 \\
45 & 0.33 & 0.149 \\
60 & 0.42 & 0.140 \\
75 & 0.57 & 0.126 \\
90 & 0.70 & 0.117 \\
105 & 0.83 & 0.108 \\
120 & 0.91 & 0.103 \\
150 & 1.23 & 0.088 \\
180 & 1.32 & 0.085 \\
\hline reaction rate r: & $0.00142(12) \pm 0.00026$ \\
\hline
\end{tabular}

van't Hoff reaction order

\begin{tabular}{cccc}
\hline $\mathrm{x}=\log (\mathrm{c})$ & $\mathrm{y}=\log (\mathrm{r})$ & $\mathrm{s}$ & $\mathrm{s}_{\mathrm{yi}}$ \\
\hline-1.62 & -2.10 & -0.032 & -0.07 \\
-1.92 & -2.27 & -0.011 & -0.024 \\
-2.22 & -2.49 & -0.043 & -0.062 \\
-2.52 & -2.85 & -0.037 & -0.079 \\
\hline \multicolumn{4}{c}{$0.82(5) \pm 0.11$} \\
\cline { 2 - 3 } & \multicolumn{2}{c}{$\mathrm{R}=0.983$} \\
\cline { 2 - 3 } & \multicolumn{2}{c}{ reaction order: }
\end{tabular}

standard conditions, concentration catalyst $=0.006 \mathrm{M}$

\begin{tabular}{ccc}
\hline time(min) & $\begin{array}{c}\text { integral ratio } \\
\mathbf{3 3 / 1}\end{array}$ & $\begin{array}{c}\text { concentration } \mathbf{1} \\
(\mathrm{M})\end{array}$ \\
\hline 2.5 & 0.01 & 0.198 \\
5 & 0.02 & 0.195 \\
7.5 & 0.04 & 0.191 \\
10 & 0.06 & 0.188 \\
15 & 0.15 & 0.173 \\
20 & 0.34 & 0.149 \\
25 & 0.43 & 0.139 \\
30 & 0.61 & 0.123 \\
40 & 0.87 & 0.106 \\
50 & 1.06 & 0.096 \\
75 & 1.81 & 0.070 \\
90 & 2.23 & 0.061 \\
\hline reaction rate r: & $0.0033(3) \pm 0.0005$ \\
\hline
\end{tabular}

standard conditions, concentration catalyst $=0.024 \mathrm{M}$

\begin{tabular}{ccc}
\hline time(min) & $\begin{array}{c}\text { integral ratio } \\
\mathbf{3 3 / 1}\end{array}$ & $\begin{array}{c}\text { concentration } \mathbf{1} \\
(\mathrm{M})\end{array}$ \\
\hline 2.5 & 0.06 & 0.189 \\
5 & 0.13 & 0.177 \\
7.5 & 0.26 & 0.158 \\
10 & 0.38 & 0.144 \\
15 & 1.06 & 0.096 \\
20 & 2.13 & 0.063 \\
25 & 3.65 & 0.042 \\
30 & 5.69 & 0.029 \\
40 & 12.50 & 0.014 \\
50 & 30.53 & 0.006 \\
60 & 64.10 & 0.003 \\
\hline reaction rate r: & $0.0080(6) \pm 0.0013$ \\
\hline \multicolumn{2}{c}{} \\
\hline
\end{tabular}


Variation of the concentration of tert-butyl hydroperoxide (Figure 12)

\begin{tabular}{ccc}
\multicolumn{3}{c}{ standard conditions, concentration peroxide $=0.0 \mathrm{M}$} \\
\hline time(min) & $\begin{array}{c}\text { integral ratio } \\
\mathbf{3 3 / 1}\end{array}$ & $\begin{array}{c}\text { concentration } \mathbf{1} \\
(\mathrm{M})\end{array}$ \\
\hline 5 & 0.00 & 0.199 \\
10 & 0.01 & 0.198 \\
15 & 0.01 & 0.198 \\
20 & 0.01 & 0.199 \\
25 & 0.03 & 0.194 \\
30 & 0.04 & 0.193 \\
40 & 0.07 & 0.186 \\
50 & 0.11 & 0.180 \\
60 & 0.14 & 0.175 \\
75 & 0.12 & 0.178 \\
90 & 0.16 & 0.172 \\
\hline reaction rate $\mathrm{r}:$ & \multicolumn{3}{|c}{$<0.00037$} \\
\hline \multicolumn{3}{c}{}
\end{tabular}

\begin{tabular}{|c|c|c|c|}
\hline time $(\min )$ & $\begin{array}{c}\text { integral ratio } \\
33 / 1\end{array}$ & $\begin{array}{c}\text { concentration } \mathbf{1} \\
\text { (M) }\end{array}$ & \\
\hline 2.5 & 0.01 & 0.198 & \\
\hline 5 & 0.06 & 0.189 & \\
\hline 7.5 & 0.08 & 0.185 & \\
\hline 10 & 0.15 & 0.173 & \\
\hline 15 & 0.40 & 0.142 & \\
\hline 20 & 0.50 & 0.132 & \\
\hline 25 & 0.58 & 0.125 & \\
\hline 30 & 1.09 & 0.094 & \\
\hline 40 & 1.78 & 0.071 & \\
\hline 50 & 2.18 & 0.062 & \\
\hline 60 & 3.31 & 0.045 & \\
\hline 75 & 4.69 & 0.034 & \\
\hline 90 & 5.22 & 0.031 & \\
\hline reaction rate $\mathrm{r}$ : & \multicolumn{2}{|c|}{$0.00367(24) \pm 0.00021$} & \\
\hline \multicolumn{4}{|c|}{ van't Hoff reaction order } \\
\hline$x=\log (c)$ & $y=\log (r)$ & $\mathrm{s}_{\mathrm{yi}}$ & $\mathrm{v}_{\mathrm{yi}}$ \\
\hline-0.70 & -1.93 & -0.007 & -0.016 \\
\hline-0.96 & -2.02 & -0.03 & -0.06 \\
\hline-1.25 & -2.27 & -0.011 & -0.024 \\
\hline-1.55 & -2.44 & -0.03 & -0.03 \\
\hline \multirow[t]{3}{*}{-1.85} & -2.66 & -0.017 & -0.014 \\
\hline & reaction order: & \multicolumn{2}{|c|}{$0.643(14) \pm 0.020$} \\
\hline & & \multicolumn{2}{|c|}{$R=0.998$} \\
\hline
\end{tabular}

standard conditions, concentration peroxide $=0.014 \mathrm{M}$

\begin{tabular}{ccc}
\hline time(min) & $\begin{array}{c}\text { integral ratio } \\
\mathbf{3 3 / 1}\end{array}$ & $\begin{array}{c}\text { concentration } \mathbf{1} \\
(\mathrm{M})\end{array}$ \\
\hline 2.5 & 0.01 & 0.198 \\
5 & 0.04 & 0.192 \\
7.5 & 0.08 & 0.184 \\
10 & 0.08 & 0.185 \\
15 & 0.15 & 0.173 \\
20 & 0.24 & 0.160 \\
25 & 0.35 & 0.147 \\
30 & 0.46 & 0.136 \\
40 & 0.68 & 0.118 \\
50 & 1.02 & 0.097 \\
60 & 1.17 & 0.091 \\
75 & 1.76 & 0.071 \\
90 & 2.22 & 0.061 \\
\hline reaction rate r: & $0.00221(9) \pm 0.00007$ \\
\hline
\end{tabular}

standard conditions, concentration peroxide $=0.11 \mathrm{M}$

\begin{tabular}{ccc}
\hline time(min) & $\begin{array}{c}\text { integral ratio } \\
\mathbf{3 3 / 1}\end{array}$ & $\begin{array}{c}\text { concentration } \mathbf{1} \\
(\mathrm{M})\end{array}$ \\
\hline 2.5 & 0.08 & 0.185 \\
5 & 0.24 & 0.160 \\
7.5 & 0.40 & 0.142 \\
10 & 0.78 & 0.111 \\
15 & 1.66 & 0.074 \\
20 & 2.75 & 0.052 \\
25 & 4.01 & 0.039 \\
30 & 5.60 & 0.030 \\
40 & 7.85 & 0.022 \\
50 & 25.26 & 0.007 \\
60 & 51.38 & 0.004 \\
75 & 104.77 & 0.002 \\
\hline
\end{tabular}

reaction rate $\mathrm{r}$ :

$0.0096(6) \pm 0.0014$

standard conditions, concentration peroxide $=0.20 \mathrm{M}$

\begin{tabular}{ccc}
\hline time(min) & $\begin{array}{c}\text { integral ratio } \\
\mathbf{3 3 / 1}\end{array}$ & $\begin{array}{c}\text { concentration } \mathbf{1} \\
(\mathrm{M})\end{array}$ \\
\hline 2.5 & 0.09 & 0.182 \\
5 & 0.29 & 0.154 \\
7.5 & 0.58 & 0.126 \\
10 & 1.09 & 0.094 \\
15 & 2.29 & 0.060 \\
20 & 3.48 & 0.044 \\
25 & 7.02 & 0.024 \\
30 & 10.03 & 0.018 \\
40 & 25.07 & 0.007 \\
50 & 63.68 & 0.003 \\
60 & 151.50 & 0.001 \\
\hline reaction rate r: & $0.01168(20) \pm 0.0004$ \\
\hline \multicolumn{2}{c}{}
\end{tabular}


Variation of the temperature (Figure 14)

\begin{tabular}{ccc}
\multicolumn{3}{l}{ standard conditions, $\mathrm{T}=285 \mathrm{~K}$} \\
\hline time $(\mathrm{min})$ & $\begin{array}{c}\text { integral ratio } \\
\mathbf{3 3 / 1}\end{array}$ & $\begin{array}{c}\text { concentration } \mathbf{1} \\
(\mathrm{M})\end{array}$ \\
\hline 2.5 & 0.00 & 0.199 \\
5 & 0.01 & 0.198 \\
7.5 & 0.03 & 0.195 \\
10 & 0.06 & 0.189 \\
15 & 0.14 & 0.175 \\
20 & 0.23 & 0.161 \\
25 & 0.29 & 0.154 \\
30 & 0.46 & 0.136 \\
40 & 0.55 & 0.127 \\
50 & 1.18 & 0.090 \\
60 & 1.64 & 0.074 \\
75 & 2.58 & 0.055 \\
90 & 3.20 & 0.046 \\
\hline reaction rate r: & $0.00234(12) \pm 0.00011$ \\
\hline
\end{tabular}

Arrhenius plot

\begin{tabular}{cccc}
\hline $\mathrm{x}=1 / \mathrm{T}$ & $\mathrm{y}=\ln (\mathrm{r})$ & $\mathrm{s}_{\mathrm{yi}}$ & $\mathrm{v}_{\mathrm{yi}}$ \\
\hline 0.00347 & -6.06 & -0.05 & -0.05 \\
0.00336 & -5.22 & -0.026 & -0.06 \\
0.00330 & -4.78 & -0.03 & -0.23 \\
0.00325 & -4.43 & $-0.22(5 \%)$ & $-0.40(10 \%)$ \\
\hline
\end{tabular}

\begin{tabular}{|c|c|}
\hline activation energy: & $60(3) \pm 6 \mathrm{KJ} / \mathrm{mol}$ \\
\hline & $\mathrm{R}=-0.9991$ \\
\hline
\end{tabular}

Kinetic isotope effect (Figure 15)

\begin{tabular}{ccc}
\multicolumn{3}{l}{ standard conditions, with $\mathrm{PhSiH}_{3}(0.20 \mathrm{M})$} \\
\hline time $(\mathrm{min})$ & $\begin{array}{c}\text { integral ratio } \\
\mathbf{3 3 / 1}\end{array}$ & $\begin{array}{c}\text { concentration } \mathbf{1} \\
(\mathrm{M})\end{array}$ \\
\hline 0 & 0.00 & 0.200 \\
2.5 & 0.17 & 0.167 \\
5 & 0.34 & 0.144 \\
7.5 & 0.56 & 0.122 \\
10 & 0.71 & 0.110 \\
15 & 1.24 & 0.083 \\
20 & 1.70 & 0.068 \\
25 & 2.18 & 0.058 \\
30 & 2.60 & 0.050 \\
40 & 3.55 & 0.040 \\
50 & 5.33 & 0.028 \\
60 & 6.36 & 0.024 \\
75 & 8.14 & 0.019 \\
90 & 11.43 & 0.014 \\
\hline reaction rate r: & $0.0103(7) \pm 0.0015$ \\
\hline
\end{tabular}

standard conditions, $\mathrm{T}=303 \mathrm{~K}$

\begin{tabular}{ccc}
\hline time(min) & $\begin{array}{c}\text { integral ratio } \\
\mathbf{3 3 / 1}\end{array}$ & $\begin{array}{c}\text { concentration } \mathbf{1} \\
(\mathrm{M})\end{array}$ \\
\hline 2.5 & 0.03 & 0.195 \\
5 & 0.09 & 0.183 \\
7.5 & 0.22 & 0.163 \\
10 & 0.40 & 0.141 \\
20 & 1.41 & 0.081 \\
25 & 1.79 & 0.069 \\
30 & 2.39 & 0.057 \\
40 & 3.57 & 0.042 \\
50 & 4.09 & 0.037 \\
60 & 6.97 & 0.024 \\
75 & 9.05 & 0.019 \\
\hline \multirow{2}{*}{ reaction rate r: } & $0.00841(26) \pm 0.0019$ \\
\hline \multicolumn{3}{c}{} \\
\hline \multicolumn{2}{c}{}
\end{tabular}

standard conditions, $\mathrm{T}=308 \mathrm{~K}$

\begin{tabular}{ccc}
\hline time(min) & $\begin{array}{c}\text { integral ratio } \\
\mathbf{3 3 / 1}\end{array}$ & $\begin{array}{c}\text { concentration } \mathbf{1} \\
(\mathrm{M})\end{array}$ \\
\hline 5 & 0.12 & 0.178 \\
7.5 & 0.34 & 0.148 \\
10 & 0.55 & 0.127 \\
15 & 1.39 & 0.082 \\
20 & 2.27 & 0.059 \\
25 & 4.13 & 0.038 \\
40 & 5.65 & 0.029 \\
50 & 5.70 & 0.028 \\
60 & 6.61 & 0.025 \\
\hline reaction rate r: & \multicolumn{2}{c}{0.012} \\
\end{tabular}

standard conditions, with $\mathrm{PhSiD}_{3}(0.20 \mathrm{M})$

\begin{tabular}{ccc}
\hline time(min) & $\begin{array}{c}\text { integral ratio } \\
\mathbf{5 4 / 1}\end{array}$ & $\begin{array}{c}\text { concentration } \mathbf{1} \\
(\mathrm{M})\end{array}$ \\
\hline 0 & 0.00 & 0.200 \\
2.5 & 0.09 & 0.183 \\
5 & 0.16 & 0.173 \\
7.5 & 0.31 & 0.151 \\
10 & 0.45 & 0.137 \\
15 & 0.64 & 0.121 \\
20 & 0.85 & 0.107 \\
25 & 0.95 & 0.102 \\
30 & 1.03 & 0.098 \\
40 & 1.47 & 0.082 \\
60 & 2.07 & 0.064 \\
\hline reaction rate r: & $0.0063(4) \pm 0.0008$ \\
\hline \multicolumn{2}{c}{} \\
\hline
\end{tabular}

kinetic isotope effect:

$.65(15) \pm 0.3$

\section{Useful Transformation of Azide and Hydrazine Products}

Conversion of Boc-Protected Hydrazines to Amine Hydrochlorides

1-Methyl-3-phenyl-propylamine hydrochloride (56)

$$
\begin{aligned}
& \text { 2) } \mathrm{Zn}, \mathrm{AcOH} \text {, acetone } \\
& 3 \quad \text { 3) } \mathrm{HCl} / \mathrm{H}_{2} \mathrm{O} \quad 56
\end{aligned}
$$


A solution of protected hydrazine 3 (364 mg, $1.00 \mathrm{mmol}, 1.00$ equiv) in acetic acid (6 mL) was refluxed for $12 \mathrm{~h}$ under argon, cooled to $23{ }^{\circ} \mathrm{C}$ and acetone $(0.6 \mathrm{~mL})$ was added. After $30 \mathrm{~min}, \mathrm{Zn}$ dust (Aldrich, $3.0 \mathrm{~g}$ ) was added, the reaction mixture was stirred for $8 \mathrm{~h}$, diluted with $\mathrm{CH}_{2} \mathrm{Cl}_{2}(10 \mathrm{~mL})$, filetered over Celite and the solvents were removed under reduced pressure. The residues were suspended in $1 \mathrm{M} \mathrm{HCl}(20 \mathrm{~mL})$, the aqueous solution was washed with $\mathrm{Et}_{2} \mathrm{O}(2 \times 15 \mathrm{~mL})$ and the pH was adusted to $>12$ with $\mathrm{NaOH}$ pellets. The basic solution was extracted with $\mathrm{Et}_{2} \mathrm{O}(3 \times 20 \mathrm{~mL})$, the organic layers were dried over $\mathrm{Na}_{2} \mathrm{SO}_{4}$ and the solvent was removed under reduced pressure ( $\left.p=100 \mathrm{mbar}, T=40^{\circ} \mathrm{C}\right)$. The crude amine was dissolved in $3 \mathrm{M} \mathrm{HCl}(20 \mathrm{~mL})$ and the solvent was removed under reduced pressure and the residues were dried for $12 \mathrm{~h}$ in high vacuo. The isolated product was recrystallized from AcOEt to afford pure amine hydrochloride 56 (95 mg, $0.51 \mathrm{mmol}, 51 \%)$ as colorless crystals. Mp (AcOEt) 142-143 ${ }^{\circ} \mathrm{C} ;{ }^{1} \mathrm{H}$ NMR (DMSO-d $\left.6,300 \mathrm{MHz}\right) \delta 8.09$ (br s, 3H, NH), 7.33-7.16 (m, 5H, Ar H), 3.11 (br m, 1H, CHN), 2.71-2.61 $\left(\mathrm{m}, 2 \mathrm{H}, \mathrm{CH}_{2}\right), 1.97-1.85\left(\mathrm{~m}, 1 \mathrm{H}, \mathrm{CH}_{2}\right), 1.77-1.66\left(\mathrm{~m}, 1 \mathrm{H}, \mathrm{CH}_{2}\right), 1.23\left(\mathrm{~d}, J=6.5 \mathrm{~Hz}, 3 \mathrm{H}, \mathrm{CH}_{3}\right) ;{ }^{13} \mathrm{C} \mathrm{NMR}\left(\mathrm{DMSO}-\mathrm{d}_{6}, 75 \mathrm{MHz}\right) \delta$ 140.8, 128.2, 128.0, 125.8, 46.3, 35.8, 30.8, 18.0; IR (KBr) v 2891 (br, s), 2615 (m), 2522 (m), 2032 (w), 1605 (m), 1517 (s), $1493(\mathrm{~m}), 1454(\mathrm{~m}), 1389$ (m), 1197 (w), 1180 (w), 1148 (w), 1130 (w), $1111(\mathrm{w}), 1068(\mathrm{w}), 1028(\mathrm{w}), 1010(\mathrm{w}), 766(\mathrm{~m}), 746$ (m), $701(\mathrm{~s}), 586(\mathrm{w}), 514(\mathrm{~m})$; MS (ESI) $150.2(\mathrm{M}-\mathrm{Cl})$; Anal. calcd for $\mathrm{C}_{10} \mathrm{H}_{16} \mathrm{NCl}$ : C, 64.68; H, 8.68; N, 7.54. Found: C, 64.43; $\mathrm{H}, 8.42 ; \mathrm{N}, 7.38$.

\section{One-pot Conversion of Olefins to Amines \\ 1-Methyl-3-phenyl-propylamine (57) ${ }^{60}$ \\ Using $\mathrm{TsN}_{3}$ (203)}

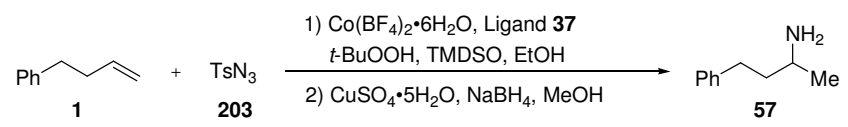

$\mathrm{Co}\left(\mathrm{BF}_{4}\right)_{2} \bullet 6 \mathrm{H}_{2} \mathrm{O}(10 \mathrm{mg}, 0.030 \mathrm{mmol}, 0.060$ equiv) and ligand 37 (14 mg, $0.030 \mathrm{mmol}, 0.060$ equiv) were dissolved in ethanol $(1.5 \mathrm{~mL})$ at $23{ }^{\circ} \mathrm{C}$ under argon. After $10 \mathrm{~min}$, 4-phenylbutene (1) $(75 \mu \mathrm{L}, 0.50 \mathrm{mmol}, 1.0$ equiv) was added to the homogenous orange solution, followed by tosyl azide $(\mathbf{2 0 3})(0.23 \mathrm{~mL}, 1.5 \mathrm{mmol}, 3.0$ equiv) and tert-butyl hydroperoxide (5.5 $\mathrm{M}$ in decane, 25 $\mu \mathrm{L}, 0.14 \mathrm{mmol}, 0.28$ equiv). After $5 \mathrm{~min}$ TMDSO $(0.18 \mathrm{~mL}, 1.0 \mathrm{mmol}, 2.0$ equiv) was added dropwise. The resulting dark brown solution was stirred at $23{ }^{\circ} \mathrm{C}$ and the reaction monitored by TLC (AcOEt/hexane 1:40). After $4 \mathrm{~h}$, the reaction mixture was cooled to $0{ }^{\circ} \mathrm{C}$ and a black suspension of $\mathrm{CuSO}_{4} \cdot 5 \mathrm{H}_{2} \mathrm{O}\left(12 \mathrm{mg}, 0.050 \mathrm{mmol}, 0.10\right.$ equiv) and $\mathrm{NaBH}_{4}(10 \mathrm{mg}, 0.26 \mathrm{mmol}, 0.50$ equiv) in methanol $(1.5 \mathrm{~mL})$ was added. ${ }^{61}$ Further $\mathrm{NaBH}_{4}\left(65 \mathrm{mg}, 1.7 \mathrm{mmol}, 3.4\right.$ equiv) was added in small portions over $45 \mathrm{~min}$ at $0{ }^{\circ} \mathrm{C}$. At this point, TLC (AcOEt/hexane 1:40) showed a clean reduction of the excess of tosyl azide, but the alkyl azide was not yet totally reduced. Further $\mathrm{NaBH}_{4}(0.10 \mathrm{~g}, 2.6 \mathrm{mmol}, 5.3$ equiv) was added in 4 portions over $2 \mathrm{~h}$, whereas TLC showed complete reduction of the alkyl azide. The reaction mixture was allowed to warm to $23{ }^{\circ} \mathrm{C}$ over $12 \mathrm{~h}$ and filtered over Celite. The Celite was washed with methanol $(20 \mathrm{~mL})$ and the solvent was removed under reduced pressure $\left(p=50 \mathrm{mbar}, T=40^{\circ} \mathrm{C}\right)$. The crude product was diluted with water $(10 \mathrm{~mL})$ and $1 \mathrm{M} \mathrm{HCl}(10 \mathrm{~mL})$ and the mixture was extracted with $\mathrm{Et}_{2} \mathrm{O}(3 \times 20 \mathrm{~mL})$. $\mathrm{NaOH}$ pellets were added to the water layer until the $\mathrm{pH}$ was higher than 10 , and the mixture was extracted with $\mathrm{Et}_{2} \mathrm{O}(3 \times 20 \mathrm{~mL})$, the organic layers were dried over $\mathrm{Na}_{2} \mathrm{SO}_{4}$, filtered and the solvent was removed under reduced pressure $\left(p=50 \mathrm{mbar}, T=40^{\circ} \mathrm{C}\right)$ to afford the free amine 57 (56 mg, $0.38 \mathrm{mmol}, 76 \%,>95 \%$ pure according to ${ }^{1} \mathrm{H} \mathrm{NMR}$ ) as a slightly yellow oil.

\section{Scale-up with Azide 46}
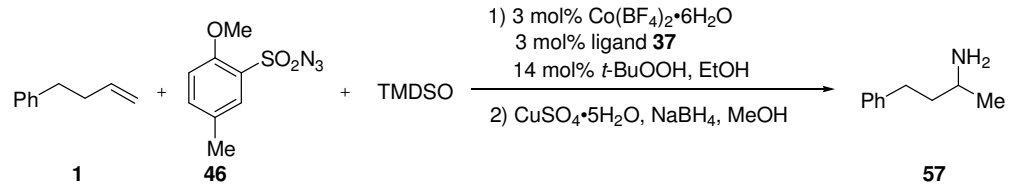

$\mathrm{Co}\left(\mathrm{BF}_{4}\right)_{2} \bullet 6 \mathrm{H}_{2} \mathrm{O}$ (50 mg, $0.15 \mathrm{mmol}, 0.030$ equiv) and ligand 37 (70 mg, $0.15 \mathrm{mmol}, 0.030$ equiv) were dissolved in ethanol (25 $\mathrm{mL})$ at $23{ }^{\circ} \mathrm{C}$ under argon. After $10 \mathrm{~min}$, 4-phenylbutene (1) $(0.75 \mathrm{~mL}, 5.0 \mathrm{mmol}, 1.0$ equiv) was added to the homogenous orange solution, followed by azide 46 ( $1.4 \mathrm{~g}, 6.2 \mathrm{mmol}, 1.2$ equiv) and tert-butyl hydroperoxide (5.5 $\mathrm{M}$ in decane, $0.13 \mathrm{~mL}, 0.71$ mmol, 0.14 equiv). After $5 \mathrm{~min}$ TMDSO $(0.88 \mathrm{~mL}, 5.0 \mathrm{mmol}, 1.0$ equiv) was added dropwise. The resulting dark green solution was stirred at $23{ }^{\circ} \mathrm{C}$ and the reaction monitored by TLC (AcOEt/hexane 1:40). After $24 \mathrm{~h}$, the reaction mixture was cooled to $0{ }^{\circ} \mathrm{C}$ and a black suspension of $\mathrm{CuSO}_{4} \cdot 5 \mathrm{H}_{2} \mathrm{O}\left(60 \mathrm{mg}, 0.24 \mathrm{mmol}, 0.050\right.$ equiv) and $\mathrm{NaBH}_{4}(50 \mathrm{mg}, 1.3 \mathrm{mmol}, 0.26$ equiv) in methanol $(10 \mathrm{~mL})$ was added. ${ }^{62}$ Further $\mathrm{NaBH}_{4}\left(0.85 \mathrm{~g}, 22 \mathrm{mmol}, 4.5\right.$ equiv) was added in small portions over $4 \mathrm{~h}$ at $0{ }^{\circ} \mathrm{C}$. At this point, TLC (AcOEt/hexane 1:40) showed complete reduction of the alkyl azide. The reaction mixture was allowed to warm to $23{ }^{\circ} \mathrm{C}$ over $12 \mathrm{~h}$ and filtered over Celite. The Celite was washed with methanol $(100 \mathrm{~mL})$ and the solvent was removed under reduced pressure $\left(p=50 \mathrm{mbar}, T=40{ }^{\circ} \mathrm{C}\right)$. The crude product was diluted with $1 \mathrm{M} \mathrm{HCl}(80 \mathrm{~mL})$ and the mixture was extracted with AcOEt $(2 \times 50 \mathrm{~mL})$ and $\mathrm{Et}_{2} \mathrm{O}(2 \times 50 \mathrm{~mL})$. $\mathrm{NaOH}$ pellets were added to the water layer until the $\mathrm{pH}$ was higher than 12 , and the mixture was extracted with $\mathrm{Et}_{2} \mathrm{O}(3 \times 100 \mathrm{~mL})$, the organic layers were dried over $\mathrm{Na}_{2} \mathrm{SO}_{4}$, filtered and the solvent was removed under reduced pressure $\left(p=50 \mathrm{mbar}, T=40^{\circ} \mathrm{C}\right)$ to afford the free amine $57(505 \mathrm{mg}, 3.38 \mathrm{mmol}, 68 \%,>95 \%$ pure according to ${ }^{1} \mathrm{H}$ NMR) as a slightly yellow oil. ${ }^{1} \mathrm{H} \mathrm{NMR}\left(\mathrm{CDCl}_{3}, 300 \mathrm{MHz}\right) \delta 7.31-7.16(\mathrm{~m}, 5 \mathrm{H}, \mathrm{Ar} \mathrm{H}), 2.96($ brs, $1 \mathrm{H}, \mathrm{CHNH}), 2.76-2.58(\mathrm{~m}$, $\left.2 \mathrm{H}, \mathrm{PhCH}_{2}\right), 1.75-1.66\left(\mathrm{~m}, 2 \mathrm{H}, \mathrm{CH}_{2} \mathrm{CHNH}_{2}\right), 1.60-1.00$ (br s, $\left.2 \mathrm{H}, \mathrm{NH}_{2}\right), 1.13\left(\mathrm{~d}, J=6.2 \mathrm{~Hz}, 3 \mathrm{H}, \mathrm{CH}_{3}\right) ;{ }^{13} \mathrm{C} \mathrm{NMR}\left(\mathrm{CDCl}_{3}, 75\right.$ MHz) $\delta 142.2,128.3,128.2,125.6,46.4,41.8,32.7,23.9$; IR v $3286(\mathrm{w}), 3061(\mathrm{w}), 3025$ (m), 2957 (s), 2925 (s), $2858(\mathrm{~m}), 1581$ (m), 1495 (m), 1454 (s), 1372 (m), 1294 (w), 1155 (w), 1100 (w), 1067 (w), 1030 (w), 907 (w), 817 (w), 747 (m), 699 (s), 579 (w), $518(\mathrm{w}), 471(\mathrm{w}), 450(\mathrm{w})$. 


\section{One-pot Conversion of Olefins to Triazoles}

1-(1-Methyl-3-phenyl-propyl)-4-phenyl-1H-[1,2,3]triazole (257)

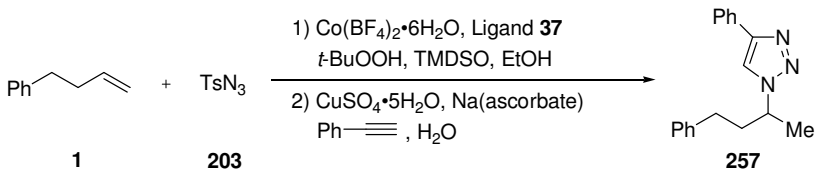

$\mathrm{Co}\left(\mathrm{BF}_{4}\right)_{2} \bullet 6 \mathrm{H}_{2} \mathrm{O}$ (10 mg, $0.030 \mathrm{mmol}, 0.060$ equiv) and ligand 37 (14 mg, $0.030 \mathrm{mmol}, 0.060$ equiv) were dissolved in ethanol $(2.5 \mathrm{~mL})$ at $23{ }^{\circ} \mathrm{C}$ under argon. After $10 \mathrm{~min}$, 4-phenylbutene (1) $(75 \mu \mathrm{L}, 0.50 \mathrm{mmol}, 1.0$ equiv) was added to the homogenous orange solution, followed by tosyl azide $(\mathbf{2 0 3})(0.23 \mathrm{~mL}, 1.5 \mathrm{mmol}, 3.0$ equiv) and tert-butyl hydroperoxide (5.5 $\mathrm{M}$ in decane, 25 $\mu \mathrm{L}, 0.14 \mathrm{mmol}, 0.28$ equiv). After $5 \mathrm{~min}$, TMDSO $(0.18 \mathrm{~mL}, 1.0 \mathrm{mmol}, 2.0$ equiv) was added dropwise. The resulting dark brown solution was stirred at $23{ }^{\circ} \mathrm{C}$ and the reaction monitored by TLC (AcOEt/hexane 1:40). After $4 \mathrm{~h}$, water (2 mL) was added. After further stirring for $30 \mathrm{~min}$, a solution of sodium ascorbate $(40 \mathrm{mg}, 0.20 \mathrm{mmol}, 0.40$ equiv) in water $(0.10 \mathrm{~mL})$, a solution of $\mathrm{CuSO}_{4} \cdot 5 \mathrm{H}_{2} \mathrm{O}(12 \mathrm{mg}, 0.050 \mathrm{mmol}, 0.10$ equiv) in water $(0.10 \mathrm{~mL})$ and phenylacetylene $(0.28 \mathrm{~mL}, 2.5 \mathrm{mmol}, 5.0$ equiv $)$ were added and the heterogenous mixture stirred at $23{ }^{\circ} \mathrm{C}$ for $20 \mathrm{~h}$. The reaction mixture was then diluted with water $(5 \mathrm{~mL})$ and brine $(5 \mathrm{~mL})$, extracted with AcOEt $(3 \times 12 \mathrm{~mL})$, the combined organic layers were dried over $\mathrm{Na}_{2} \mathrm{SO}_{4}$, filtered and the solvent was removed under reduced pressure. The isolated product was purified by column chromatography (solvent gradient: pentane $/ \mathrm{CH}_{2} \mathrm{Cl}_{2} / \mathrm{Et}_{2} \mathrm{O}$ 50:100:5, then pentane $\left./ \mathrm{CH}_{2} \mathrm{Cl}_{2} / \mathrm{Et}_{2} \mathrm{O} 50: 100: 7.5\right)$ to afford 257 (83 mg, $\left.0.30 \mathrm{mmol}, 60 \%\right)$ as a slightly yellow solid. Analitically pure samples of 257 were obtained after recrystallization from AcOEt/hexane. $\mathrm{R}_{\mathrm{f}}\left(\mathrm{pentane} / \mathrm{CH}_{2} \mathrm{Cl}_{2} /\right.$ $\mathrm{Et}_{2} \mathrm{O}$ 50:100:7.5) 0.30; $\mathrm{Mp}$ (AcOEt/hexane) $72-73{ }^{\circ} \mathrm{C} ;{ }^{1} \mathrm{H} \mathrm{NMR}\left(\mathrm{CDCl}_{3}, 300 \mathrm{MHz}\right) \delta 7.88-7.84(\mathrm{~m}, 2 \mathrm{H}, \mathrm{Ar} \mathrm{H}), 7.75(\mathrm{~s}, 1 \mathrm{H}$, triazole H), 7.46-7.13 (m, 8H, Ar H), 4.75-4.68 (m, 1H, CHN), 2.57 (t, J = 7.6 Hz, 2H, $\left.\mathrm{PhCH}_{2}\right), 2.41-2.14\left(\mathrm{~m}, 2 \mathrm{H}, \mathrm{CH} \mathrm{CHN}^{\mathrm{CH}}\right.$, $1.63\left(\mathrm{~d}, J=6.8 \mathrm{~Hz}, \mathrm{CH}_{3}\right) ;{ }^{13} \mathrm{C} \mathrm{NMR}\left(\mathrm{CDCl}_{3}, 75 \mathrm{MHz}\right) \delta 147.4,140.2,130.6,128.7,128.4,128.3,127.9,126.1,125.5,117.4$, 56.7, 38.7, 32.1, 21.7; IR v 3130 (w), $3084(w), 3061$ (w), $3026(w), 2978(w), 2935$ (w), $2860(w), 1951(w), 1883(w), 1812(w)$, $1604(\mathrm{w}), 1580(\mathrm{w}), 1553(\mathrm{w}), 1495(\mathrm{w}), 1482(\mathrm{~m}), 1454(\mathrm{~m}), 1436(\mathrm{~m}), 1383(\mathrm{w}), 1364(\mathrm{w}), 1226(\mathrm{w}), 1180(\mathrm{w}), 1156(\mathrm{w}), 1119$ (w), $1074(\mathrm{~m}), 1028(\mathrm{w}), 973(\mathrm{w}), 914(\mathrm{w}), 765(\mathrm{~s}), 749(\mathrm{~m}), 696(\mathrm{~s})$; HRMS (EI) calcd for $\mathrm{C}_{18} \mathrm{H}_{19} \mathrm{~N}_{3}{ }^{+}(\mathrm{M}): 277.1579$, found 277.1572; Anal. calcd for $\mathrm{C}_{18} \mathrm{H}_{19} \mathrm{~N}_{3}$ : C, 77.95; H, 6.90; N, 15.15. Found: C, 77.87; H, 6.93; N, 15.11 .

\section{Case Study: Amination of Methylenecyclobutane and Methylenecyclopropane}

$N$-(1-Methyl-cyclobutyl)- $N$ '-(tert-butoxycarbonyl)hydrazinecarboxylic acid tert-butyl ester (60)

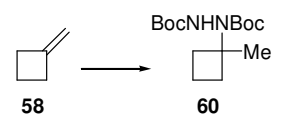

Following GP1 Hydrohydrazination product 60 (540 mg, $1.80 \mathrm{mmol}$, 90\%) was obtained as a colorless solid with methylenecyclobutane (58) $(0.18 \mathrm{~mL}, 2.0 \mathrm{mmol}, 1.0$ equiv) in $5 \mathrm{~h}$ with $5 \mathrm{~mol} \%$ catalyst 20 after purification by column chromatography (AcOEt/hexane 1:10). Following GP3 Hydrohydrazination product 60 (263 mg, 0.876 mmol, 88\%) was obtained as a colorless solid together with the regioisomeric product $(<26 \mathrm{mg},<0.087 \mathrm{mmol},<9 \%$, not isolated $)$ with methylenecyclobutane (58) $(92 \mu \mathrm{L}, 1.0 \mathrm{mmol}, 1.0$ equiv) in $2 \mathrm{~h}$ with $2 \mathrm{~mol} \%$ catalyst 23 after purification by column chromatography (AcOEt/hexane 1:10). $\mathrm{R}_{\mathrm{f}}(\mathrm{AcOEt} / \mathrm{h}$ xane $1: 10) 0.20 ; \mathrm{Mp} \mathrm{118-119}{ }^{\circ} \mathrm{C} ;{ }^{1} \mathrm{H} \mathrm{NMR}\left(\mathrm{CDCl}_{3}, 300 \mathrm{MHz}, 52{ }^{\circ} \mathrm{C}\right) \delta 6.06$ (br s, $1 \mathrm{H}, \mathrm{NH}), 2.39-2.29\left(\mathrm{~m}, 2 \mathrm{H}, \mathrm{CH}_{2}\right), 1.89-1.84\left(\mathrm{~m}, 2 \mathrm{H}, \mathrm{CH}_{2}\right), 1.76-1.65\left(\mathrm{~m}, 2 \mathrm{H}, \mathrm{CH}_{2}\right), 1.48\left(\mathrm{~s}, 9 \mathrm{H}, \mathrm{OCCH}_{3}\right), 1.46(\mathrm{~s}, 9 \mathrm{H}$, $\left.\mathrm{OCCH}_{3}\right), 1.41\left(\mathrm{~s}, 3 \mathrm{H}, \mathrm{NCCH}_{3}\right) ;{ }^{13} \mathrm{C} \mathrm{NMR}\left(\mathrm{CDCl}_{3}, 75 \mathrm{MHz}, 52{ }^{\circ} \mathrm{C}\right) \delta 156.0,154.0,80.9,80.7,61.1,33.6,28.5,28.3,23.0,13.8$; IR v $3324(\mathrm{~m}), 2978(\mathrm{~s}), 1706(\mathrm{~s}), 1480(\mathrm{~m}), 1456$ (m), 1428 (w), 1367 (s), 1250 (s), 1163 (s), 1086 (m), 1051 (m), 1017 (m), 919 (w), $876(w), 856(w), 780(w), 760(\mathrm{~m}), 734(\mathrm{~m}), 691(\mathrm{w}), 647(\mathrm{w}), 602(\mathrm{w}), 489(\mathrm{w}), 462(\mathrm{w}) ;$ MS (ESI) $301.2(\mathrm{M}+\mathrm{H}), 323.2$ $(\mathrm{M}+\mathrm{Na})$; Anal. calcd for $\mathrm{C}_{15} \mathrm{H}_{28} \mathrm{~N}_{2} \mathrm{O}_{4}$ : C, 59.98, H, 9.39; N, 9.33. Found: C, 60.25; H, 9.50, N, 9.16.

\section{1-Methylcyclobutylamine hydrochloride (62)}

From Hydrazine 60

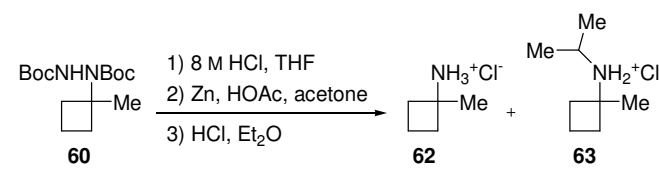

Hydrohydrazination product 60 (150 mg, $0.500 \mathrm{mmol}, 1.00$ equiv) was dissolved in THF $(1.5 \mathrm{~mL})$ and $8 \mathrm{M} \mathrm{HCl}(4.5 \mathrm{~mL})$ was added, whereas partial precipitation of $\mathbf{6 0}$ occurred. The reaction mixture was stirred vigorously for $1 \mathrm{~h}$ to give a clear solution. The solvents were removed under reduced pressure and the residues dried $3 \mathrm{~h}$ in high vacuo. Acetic acid $(3 \mathrm{~mL})$ and $\mathrm{Zn}$ dust (Acros, freshly activated ( $\mathrm{Zn}$ dust $(30 \mathrm{~g})$ was stirred with $5 \% \mathrm{HCl}(40 \mathrm{~mL})$ for $3 \mathrm{~min}$, filtered and washed with water $(3 \times 40 \mathrm{~mL})$, acetone $(2 \times 30 \mathrm{~mL})$ and $\mathrm{Et}_{2} \mathrm{O}(2 \times 30 \mathrm{~mL})$ and dried $2 \mathrm{~h}$ in high vacuo $\left.), 1.0 \mathrm{~g}\right)$ were added, followed 10 min later by acetone $(0.3$ $\mathrm{mL})$. After $2 \mathrm{~h}$, the reaction was filtered over Celite, the Celite was washed with $\mathrm{MeOH}(40 \mathrm{~mL})$, the $\mathrm{pH}$ was adjusted to $1 \mathrm{with}$ $37 \% \mathrm{HCl}$, and the solvents were removed under reduced pressure. The residues were suspended in $1 \mathrm{M} \mathrm{HCl}(20 \mathrm{~mL})$, the suspension was washed with $\mathrm{Et}_{2} \mathrm{O}(3 \times 15 \mathrm{~mL})$ and the $\mathrm{pH}$ was adjusted to 12 with $\mathrm{NaOH}$ pellets. The resulting solution was extracted with $\mathrm{Et}_{2} \mathrm{O}(2 \times 20 \mathrm{~mL})$, saturated in $\mathrm{NaCl}$ and extracted again with $\mathrm{Et}_{2} \mathrm{O}(20 \mathrm{~mL})$. The combined organic layers were dried over $\mathrm{Na}_{2} \mathrm{SO}_{4}$, filtred, $\mathrm{HCl}\left(2 \mathrm{M}\right.$ in $\mathrm{Et}_{2} \mathrm{O}, 2.5 \mathrm{~mL}$ ) was added and the solvent was removed under reduced pressure. The 
residues were co-evaporated with toluene $(2 \mathrm{x})$ and $\mathrm{CH}_{2} \mathrm{Cl}_{2}(2 \mathrm{x})$ and dried $2 \mathrm{~h}$ in high vacuo. The isolated product was recrystallized from AcOEt/MeOH to yield amine hydrochloride $62(51 \mathrm{mg}, 0.42 \mathrm{mmol}, 84 \%)$ together with the reductive amination product $63(6 \mathrm{mg}, 0.04 \mathrm{mmol}, 7 \%)$. Complete separation of 62 and $\mathbf{6 3}$ could not be achieved.

From Methylenecyclobutane 62 via Hydroazidation and Reduction

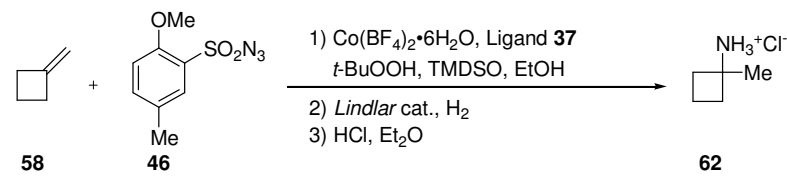

$\mathrm{Co}\left(\mathrm{BF}_{4}\right)_{2} \cdot 6 \mathrm{H}_{2} \mathrm{O}$ (40 mg, $0.12 \mathrm{mmol}, 0.060$ equiv) and ligand 37 (56 mg, $0.12 \mathrm{mmol}, 0.060$ equiv) were dissolved in ethanol (10 $\mathrm{mL})$ at $23{ }^{\circ} \mathrm{C}$ under argon. After $10 \mathrm{~min}$, the reaction was cooled to $0{ }^{\circ} \mathrm{C}$ and methylenecyclobutane $(\mathbf{5 8})(0.18 \mathrm{~mL}, 2.0 \mathrm{mmol}, 1.0$ equiv) was added to the homogenous orange solution, followed by azide 46 (0.68 g, $3.1 \mathrm{mmol}$, 1.5 equiv) and tert-butyl hydroperoxide $(5.5 \mathrm{M}$ in decane, $0.10 \mathrm{~mL}, 0.55 \mathrm{mmol}, 0.28$ equiv). After $5 \mathrm{~min}$, TMDSO (0.52 mL, $3.0 \mathrm{mmol}, 1.5 \mathrm{equiv})$ was added dropwise to the suspension. The resulting dark brown-green solution was allowed to warm to $23{ }^{\circ} \mathrm{C}$ over $2 \mathrm{~h}$ and stirred at $23{ }^{\circ} \mathrm{C}$ until the color changed back to orange-red $(6 \mathrm{~h})$. At this point, Lindlar catalyst $(5 \% \mathrm{Pd}, 0.36 \mathrm{~g})$ was added and the reaction was put under $\mathrm{H}_{2}$ (1 atm, balloon). After $13 \mathrm{~h}$, the reaction was filtered over Celite, the Celite was washed with $\mathrm{MeOH}(40 \mathrm{~mL})$, the $\mathrm{pH}$ was adjusted to 1 with $37 \% \mathrm{HCl}$, and the solvents were removed under reduced pressure. The residues were suspended in $1 \mathrm{M} \mathrm{HCl}(20 \mathrm{~mL})$, the suspension was washed with $\mathrm{Et}_{2} \mathrm{O}(3 \mathrm{x} 15 \mathrm{~mL})$ and the $\mathrm{pH}$ was adjusted to 12 with $\mathrm{NaOH}$ pellets. The resulting solution was extracted with $\mathrm{Et}_{2} \mathrm{O}(2 \times 20 \mathrm{~mL})$, saturated in $\mathrm{NaCl}$ and extracted again with $\mathrm{Et}_{2} \mathrm{O}(20 \mathrm{~mL})$. The combined organic layers were dried over $\mathrm{Na}_{2} \mathrm{SO}_{4}$, filtred, $\mathrm{HCl}\left(2 \mathrm{M}\right.$ in $\left.\mathrm{Et}_{2} \mathrm{O}, 2.5 \mathrm{~mL}\right)$ was added and the solvent was removed under reduced pressure. The residues were co-evaporated with toluene $(2 \mathrm{x})$ and $\mathrm{CH}_{2} \mathrm{Cl}_{2}(2 \mathrm{x})$ and dried $2 \mathrm{~h}$ in high vacuo. The isolated product was recrystallized from AcOEt/MeOH to yield amine hydrochloride 62 (145 mg, $1.19 \mathrm{mmol}, 60 \%)$ as colorless crystals. Mp (AcOEt/MeOH) 241-244 ${ }^{\circ} \mathrm{C} ;{ }^{1} \mathrm{H}$ NMR $\left(\mathrm{CD}_{3} \mathrm{OD}, 300 \mathrm{MHz}\right) \delta 4.91\left(\mathrm{~m},>3 \mathrm{H}, \mathrm{NH}\right.$ and $\left.\mathrm{H}_{2} \mathrm{O}\right), 2.36-2.27\left(\mathrm{~m}, 2 \mathrm{H}, \mathrm{CH}_{2}\right), 2.11-1.90$ $\left(\mathrm{m}, 4 \mathrm{H}, \mathrm{CH}_{2}\right), 1.50\left(\mathrm{~s}, 3 \mathrm{H}, \mathrm{CH}_{3}\right) ;{ }^{13} \mathrm{C} \mathrm{NMR}\left(\mathrm{CD}_{3} \mathrm{OD}, 75 \mathrm{MHz}\right) \delta 54.7,33.0,23.9,13.8$; IR (KBr) v $2963(\mathrm{~s}$, br), $2762(\mathrm{~s}), 2696$ $(\mathrm{m}), 2640(\mathrm{~m}), 2593(\mathrm{~m}), 2539(\mathrm{~m}), 2506(\mathrm{~m}), 2054(\mathrm{~m}), 1920(\mathrm{w}), 1604(\mathrm{w}), 1504(\mathrm{~s}), 1456(\mathrm{w}), 1428(\mathrm{w}), 1381(\mathrm{~s}), 1323(\mathrm{w})$, 1270 (s), 1232 (w), 1206 (w), 1175 (m), 1097 (w), 1051 (w), $980(w), 924$ (w), 905 (w), 712 (w), 608 (w), 417 (m); MS (ESI) 86.4 (M-Cl); Anal. calcd for $\mathrm{C}_{5} \mathrm{H}_{12} \mathrm{NCl}$ : C, 49.38; H, 9.95; N, 11.52. Found: C, 49.31; H, 9.66; N, 11.33.

\section{1-(1-Methyl-cyclobutyl)-4-phenyl-1H-[1,2,3]triazole (65)}

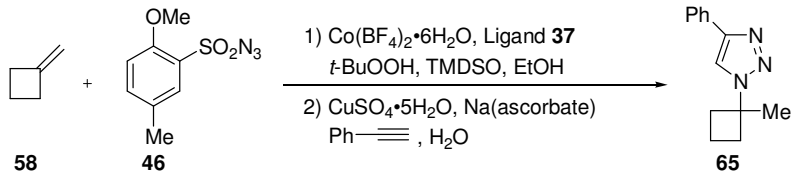

$\mathrm{Co}\left(\mathrm{BF}_{4}\right)_{2} \bullet 6 \mathrm{H}_{2} \mathrm{O}$ (20 mg, $0.060 \mathrm{mmol}, 0.060$ equiv) and ligand 37 (28 mg, $0.060 \mathrm{mmol}, 0.060$ equiv) were dissolved in ethanol $(2.5 \mathrm{~mL})$ at $23{ }^{\circ} \mathrm{C}$ under argon. After $10 \mathrm{~min}$, the reaction was cooled to $0{ }^{\circ} \mathrm{C}$ and methylenecyclobutane $(\mathbf{5 8})(92 \mu \mathrm{L}, 1.0 \mathrm{mmol}$, 1.0 equiv) was added to the homogenous orange solution, followed by azide 46 ( $0.34 \mathrm{~g}, 1.5 \mathrm{mmol}, 1.5$ equiv) and tert-butyl hydroperoxide $(5.5 \mathrm{M}$ in decane, $50 \mu \mathrm{L}, 0.28 \mathrm{mmol}, 0.28$ equiv). After $5 \mathrm{~min}$, TMDSO $(0.26 \mathrm{~mL}, 1.5 \mathrm{mmol}, 1.5$ equiv) was added dropwise to the suspension. The resulting dark brown-green solution was allowed to warm to $23{ }^{\circ} \mathrm{C}$ over $2 \mathrm{~h}$ and stirred at $23{ }^{\circ} \mathrm{C}$ until the color changed back to orange-red $(6 \mathrm{~h})$. Water $(3.5 \mathrm{~mL})$ was added. After further stirring for 30 min, a solution of sodium ascorbate $\left(80 \mathrm{mg}, 0.40 \mathrm{mmol}, 0.40\right.$ equiv) in water $(0.20 \mathrm{~mL})$, a solution of $\mathrm{CuSO}_{4} \cdot 5 \mathrm{H}_{2} \mathrm{O}(24 \mathrm{mg}, 0.10 \mathrm{mmol}, 0.10$ equiv) in water $(0.20 \mathrm{~mL})$ and phenylacetylene (distilled, $0.28 \mathrm{~mL}, 2.5 \mathrm{mmol}, 2.5$ equiv) were added and the heterogenous mixture stirred at 23 ${ }^{\circ} \mathrm{C}$ for $20 \mathrm{~h}$. The reaction mixture was then diluted with water $(5 \mathrm{~mL})$ and brine $(5 \mathrm{~mL})$, extracted with AcOEt $(3 \times 15 \mathrm{~mL})$, the combined organic layers were dried over $\mathrm{Na}_{2} \mathrm{SO}_{4}$, filtered and the solvent was removed under reduced pressure. The isolated product was purified by column chromatography (solvent gradient: pentane $/ \mathrm{CH}_{2} \mathrm{Cl}_{2} / \mathrm{Et}_{2} \mathrm{O} 50: 100: 5$, then pentane $/ \mathrm{CH}_{2} \mathrm{Cl}_{2} / \mathrm{Et}_{2} \mathrm{O}$ 50:100:7.5) to afford $\mathbf{6 5}(114 \mathrm{mg}, 0.53 \mathrm{mmol}, 53 \%)$ as a slightly yellow solid. Analitically pure samples of $\mathbf{6 5}$ were obtained after recrystallization from hexane. $\mathrm{R}_{\mathrm{f}}$ (pentane/ $\left.\mathrm{CH}_{2} \mathrm{Cl}_{2} / \mathrm{Et}_{2} \mathrm{O} 50: 100: 7.5\right)$ 0.25; $\mathrm{Mp}$ (Hexane) $102-104{ }^{\circ} \mathrm{C} ;{ }^{1} \mathrm{H} \mathrm{NMR}\left(\mathrm{CDCl}{ }_{3}, 300 \mathrm{MHz}\right.$, )

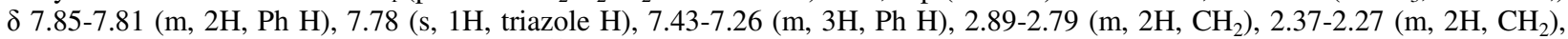
2.07-1.95 (m, 2H, $\left.\mathrm{CH}_{2}\right), 1.79\left(\mathrm{~s}, 3 \mathrm{H}, \mathrm{CH}_{3}\right) ;{ }^{13} \mathrm{C} \mathrm{NMR}\left(\mathrm{CDCl}_{3}, 75 \mathrm{MHz}\right.$, $) \delta 147.2,130.7,128.6,127.7,125.5,116.7,61.0,34.8$, 26.7, 14.2; IR v $3086(\mathrm{w}), 2976(\mathrm{~m}), 2943(\mathrm{~m}), 1610(\mathrm{w}), 1483(\mathrm{~m}), 1456(\mathrm{~m}), 1413(\mathrm{~m}), 1381(\mathrm{w}), 1352(\mathrm{w}), 1261(\mathrm{~m}), 1227(\mathrm{~m})$, $1202(\mathrm{~m}), 1075$ (m), 1027 (m), 973 (w), 921 (w), 827 (w), 768 (s), 697 (s), 513 (w); MS (ESI) 214.3 (M+H), 236.3 (M+Na); Anal. calcd for $\mathrm{C}_{13} \mathrm{H}_{15} \mathrm{~N}_{3}$ : C, 73.21; H, 7.09; N, 19.70. Found: C, 73.30; H, 7.19; N, 19.42.

\section{$N$-(1-Methyl-cyclopropyl)- $N$ '-(tert-butoxycarbonyl)hydrazinecarboxylic acid tert-butyl ester (66)}

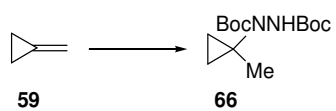

Following GP3 Hydrohydrazination product $66(439 \mathrm{mg}, 1.53 \mathrm{mmol}, 77 \%)$ was obtained as a colorless solid with methylenecyclopropane (59) $(0.15 \mathrm{~mL}, 2.0 \mathrm{mmol}, 2.0$ equiv) in $12 \mathrm{~h}$ with $2 \mathrm{~mol} \%$ catalyst 23 after purification by column chromatography (AcOEt/hexane 1:10-1:8). $\mathrm{R}_{\mathrm{f}}$ (AcOEt/hexane 1:5) 0.30; Mp 126-127 ${ }^{\circ} \mathrm{C} ;{ }^{1} \mathrm{H} \mathrm{NMR}\left(\mathrm{CDCl}_{3}, 300 \mathrm{MHz}, 52{ }^{\circ} \mathrm{C}\right) \delta$ 6.23 (br s, $1 \mathrm{H}, \mathrm{NH}), 1.48\left(\mathrm{~s}, 18 \mathrm{H}, \mathrm{OCCH}_{3}\right), 1.33\left(\mathrm{~s}, 3 \mathrm{H}, \mathrm{NCCH}_{3}\right), 0.99\left(\mathrm{br} \mathrm{m}, 2 \mathrm{H}, \mathrm{CH}_{2}\right), 0.67-0.59\left(\mathrm{~m}, 2 \mathrm{H}, \mathrm{CH}_{2}\right) ;{ }^{13} \mathrm{C} \mathrm{NMR}^{\mathrm{N}}$ $\left(\mathrm{CDCl}_{3}, 75 \mathrm{MHz}, 52{ }^{\circ} \mathrm{C}\right) \delta 155.6,80.8,38.6,28.4,28.2,21.2,15.9 ;$ IR $v 3328(\mathrm{~m}), 2978(\mathrm{~m}), 2933(\mathrm{~m}), 1715(\mathrm{~s}), 1480(\mathrm{~m}), 1456$ 
(w), 1385 (s), 1367 (s), 1248 (s), 1166 (s), $1118(\mathrm{~m}), 1088(\mathrm{~m}), 1048(\mathrm{~m}), 1020(\mathrm{~m}), 952(\mathrm{w}), 914(\mathrm{w}), 854(\mathrm{w}), 785(\mathrm{w}), 762$ (w), 735 (w), 669 (w), 647 (w), $591(w), 534(w)$; MS (ESI) $287.3(\mathrm{M}+\mathrm{H}), 309.2(\mathrm{M}+\mathrm{Na})$; Anal. calcd for $\mathrm{C}_{14} \mathrm{H}_{26} \mathrm{~N}_{2} \mathrm{O}_{4}: \mathrm{C}, 58.72, \mathrm{H}$, 9.15; N, 9.78. Found: C, 58.51; H, 9.08, N, 9.60.

\section{1-Methylcyclopropylamine hydrochloride (68)}

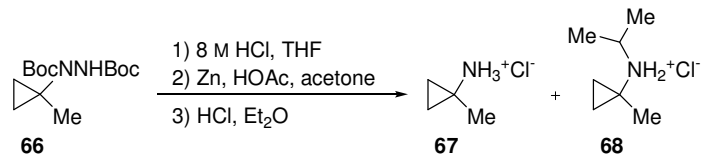

Hydrohydrazination product 66 (509 mg, $1.78 \mathrm{mmol}, 1.00$ equiv) was dissolved in THF (2 mL) and $8 \mathrm{M} \mathrm{HCl}(6 \mathrm{~mL})$ was added, whereas partial precipitation of $\mathbf{6 6}$ occurred. The reaction mixture was stirred vigorously for $1 \mathrm{~h}$ to give a clear solution. The solvents were removed under reduced pressure and the residues dried $3 \mathrm{~h}$ in high vacuo. Acetic acid (10 $\mathrm{mL})$ and $\mathrm{Zn}$ dust (Acros, freshly activated ( $\mathrm{Zn}$ dust $(30 \mathrm{~g})$ was stirred with $5 \% \mathrm{HCl}(40 \mathrm{~mL})$ for $3 \mathrm{~min}$, filtered and washed with water $(3 \times 40 \mathrm{~mL})$, acetone $(2 \times 30 \mathrm{~mL})$ and $\mathrm{Et}_{2} \mathrm{O}(2 \times 30 \mathrm{~mL})$ and dried $2 \mathrm{~h}$ in high vacuo $\left.), 2.0 \mathrm{~g}\right)$ were added, followed 10 min later by acetone $(1.0 \mathrm{~mL})$. After $1 \mathrm{~h}$, the reaction was filtered over Celite, the Celite was washed with $\mathrm{MeOH}(40 \mathrm{~mL})$, the $\mathrm{pH}$ was adjusted to 1 with $37 \% \mathrm{HCl}$, and the solvents were removed under reduced pressure. The residues were suspended in $1 \mathrm{M} \mathrm{HCl}(20 \mathrm{~mL})$, the suspension was washed with $\mathrm{Et}_{2} \mathrm{O}(3 \times 15 \mathrm{~mL})$ and the $\mathrm{pH}$ was adjusted to 12 with $\mathrm{NaOH}$ pellets. The resulting solution was extracted with $\mathrm{Et}_{2} \mathrm{O}$ $(2 \times 20 \mathrm{~mL})$, saturated in $\mathrm{NaCl}$ and extracted again with $\mathrm{Et}_{2} \mathrm{O}(20 \mathrm{~mL})$. The combined organic layers were dried over $\mathrm{Na}_{2} \mathrm{SO}_{4}$, filtred, $\mathrm{HCl}\left(2 \mathrm{M}\right.$ in $\left.\mathrm{Et}_{2} \mathrm{O}, 2.5 \mathrm{~mL}\right)$ was added and the solvent was removed under reduced pressure. The residues were coevaporated with toluene $(2 \mathrm{x})$ and $\mathrm{CH}_{2} \mathrm{Cl}_{2}(2 \mathrm{x})$ and dried $2 \mathrm{~h}$ in high vacuo. The isolated product was recrystallized from AcOEt/MeOH to yield amine hydrochloride 67 (1. crop: pure 67 (90 mg, 0.84 mmol, 47\%), 2.crop: 67 (48 mg, $0.45 \mathrm{mmol}, 25 \%$ ) and $68(4 \mathrm{mg}, 0.03 \mathrm{mmol}, 2 \%)$, combined yield of 67: 72\%) as colorless crystals. Mp (AcOEt/MeOH) $204-205{ }^{\circ} \mathrm{C}$; ${ }^{1} \mathrm{H} \mathrm{NMR}$ $\left(\mathrm{CD}_{3} \mathrm{OD}, 300 \mathrm{MHz}\right) \delta 4.87\left(\mathrm{~m},>3 \mathrm{H}, \mathrm{NH}\right.$ and $\left.\mathrm{H}_{2} \mathrm{O}\right), 1.45\left(\mathrm{~s}, 3 \mathrm{H}, \mathrm{CH}_{3}\right), 0.95-0.90\left(\mathrm{~m}, 2 \mathrm{H}, \mathrm{CH}_{2}\right), 0.77-0.73\left(\mathrm{~m}, 2 \mathrm{H}, \mathrm{CH}_{2}\right) ;{ }^{13} \mathrm{C}$ NMR (CD $\left.{ }_{3} \mathrm{OD}, 75 \mathrm{MHz}\right) \delta$ 31.6, 20.8, 11.3; IR (KBr) v 2906 (s, br), 2695 (s), 2595 (s), 2516 (s), 2432 (m), 2063 (s), 1950 (w), 1806 (w), 1610 (m), 1520 (s), 1470 (m), 1451 (s), 1424 (s), 1395 (s), 1363 (w), 1275 (s), 1208 (m), 1112 (w), 1061 (w), 1027 (s), $1002(\mathrm{w}), 942(\mathrm{w}), 920(\mathrm{w}), 870(\mathrm{~m}), 770(\mathrm{w}), 684(\mathrm{w}), 431$ (s), $410(\mathrm{~s})$; MS (ESI) 72.5 (M-Cl); Anal. calcd for $\mathrm{C}_{4} \mathrm{H}_{10} \mathrm{NCl}$ : C, 44.66; H, 9.37; N, 13.02. Found: C, 44.56; H, 9.18; N, 12.92.

\section{Important Spectra for Characterisation}



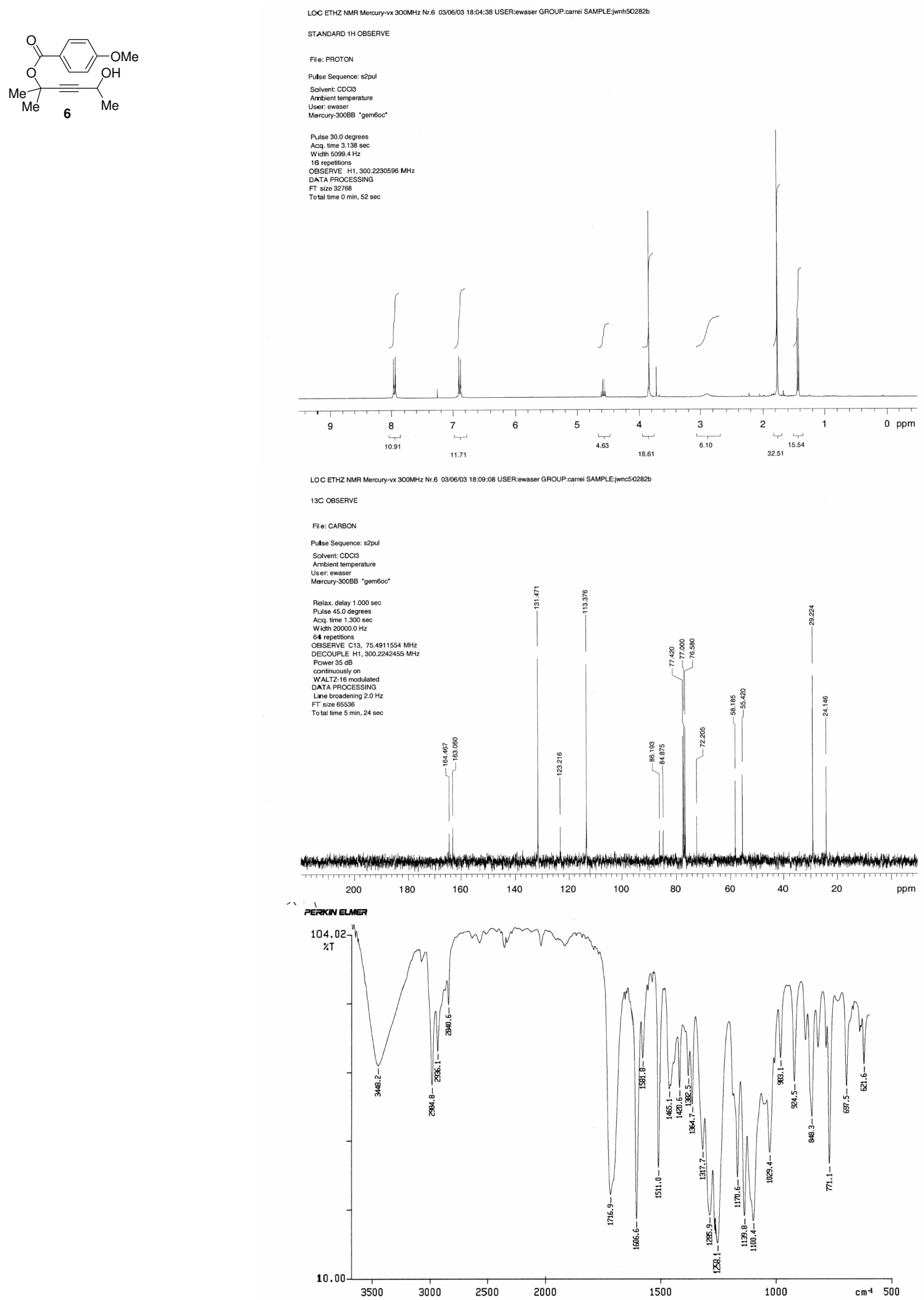

03/06/22 19:07 jerome

$X: 4$ scans, $4.0 \mathrm{~cm}^{-1}$ 

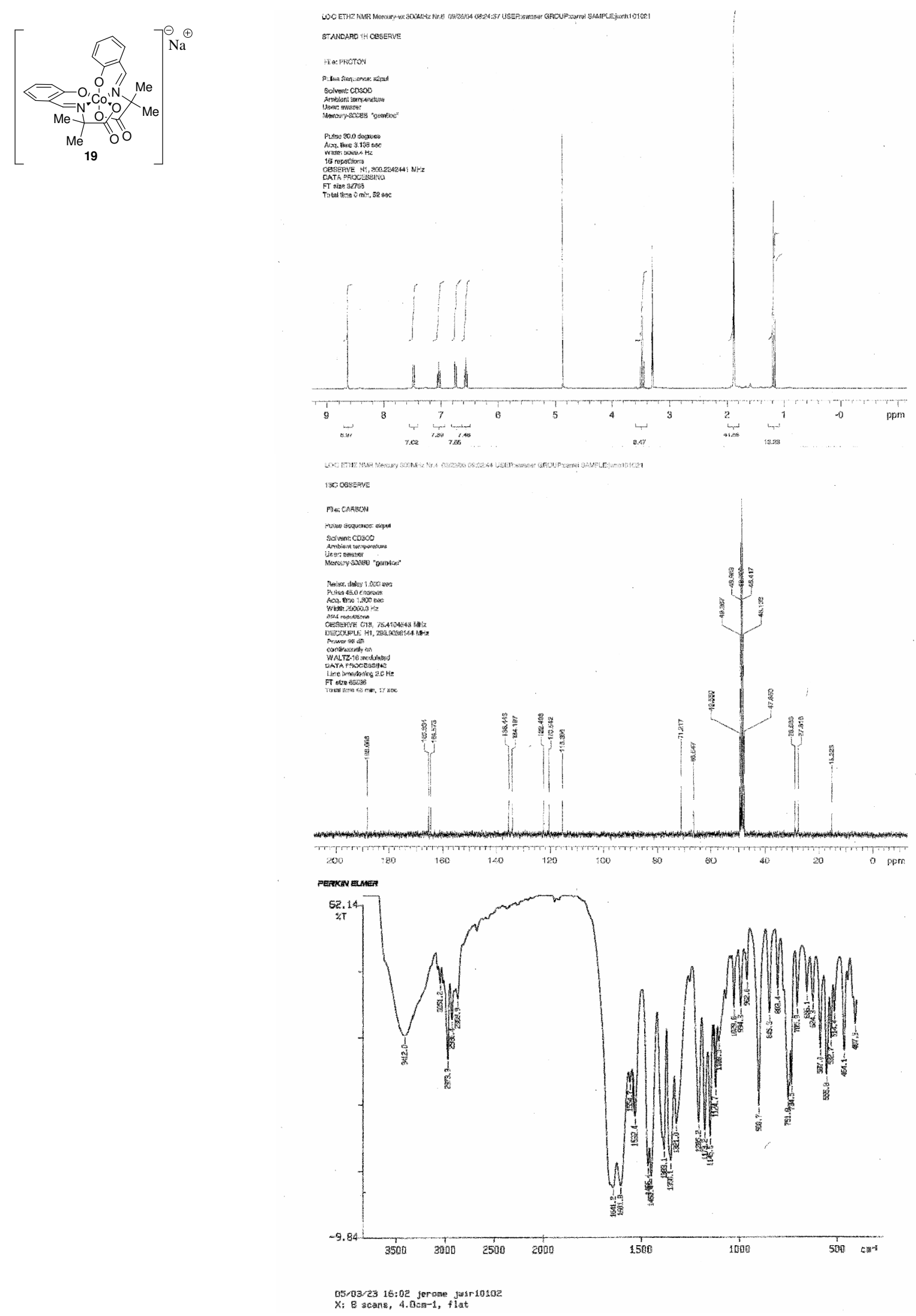